\title{
A Semantic Implementation for Data Management in an Open Building Automation Platform
}

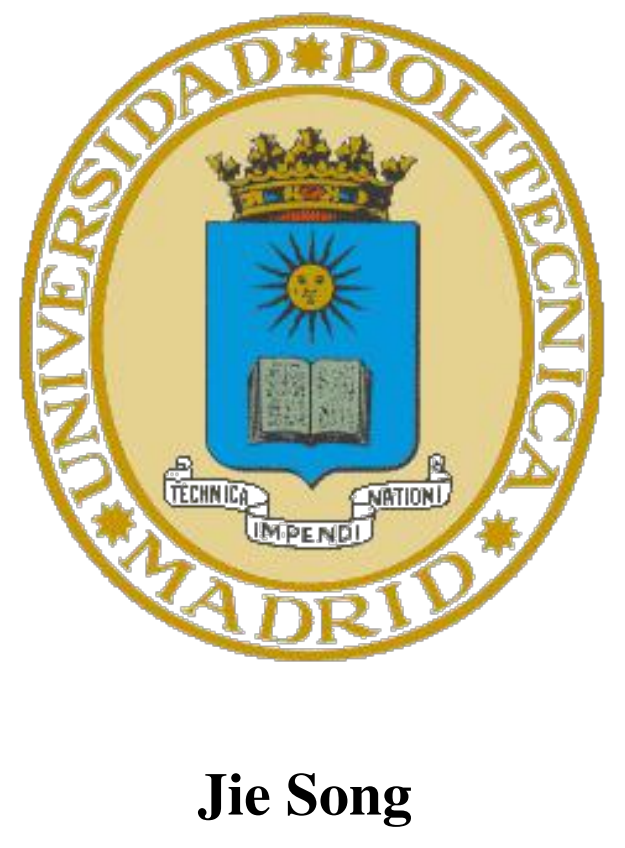

Supervisor: Prof. Asunción Santamaría Galdón

Escuela Técnica Superior de Ingenieros Informáticos Universidad Politécnica de Madrid (UPM)

This dissertation is submitted for the degree of Doctor of Artificial Intelligence 



\section{TRIBUNAL}

Tribunal nombrado por el Magnífico y Excelentísimo Sr. Rector de la Universidad Politécnica de Madrid.

$\begin{array}{lll}\text { Presidente } & \text { Dr. } & \text { D. } \\ \text { Vocal } & \text { Dr. } & \text { D. } \\ \text { Vocal } & \text { Dr. } & \text { D. } \\ \text { Vocal } & \text { Dr. } & \text { D. } \\ \text { Secretario } & \text { Dr. } & \text { D. }\end{array}$

Realizado el acto de lectura y defensa de la Tesis el día de Septiembre de 2017 en Madrid.

Calificación

Presidente

Vocal

Secretario
Vocal

Vocal 

I would like to dedicate this thesis to my loving parents ... 



\section{Declaration}

I hereby declare that except where specific reference is made to the work of others, the contents of this dissertation are original and have not been submitted in whole or in part for consideration for any other degree or qualification in this, or any other university. This dissertation is my own work and contains nothing which is the outcome of work done in collaboration with others, except as specified in the text and Acknowledgements.

Jie Song

May 2017 



\section{Acknowledgements}

Six years ago, I wanted to give myself some challenges and decided to come to Spain for $\mathrm{PhD}$ study. The beginning was hard, either in the sense of language or research. I have to say that I am really lucky that I have met lots of good people who have helped me a lot.

I would like to give my sincere thanks to all my colleagues from CeDInt, the classmates and professors from UPM, the colleagues from ETH Zurich and my friends who have been always supporting me.

First, I would like to give my special thank to my supervisor Asun, for providing me the opportunity to work at CeDInt and all the help during the preparation of this thesis.

Second, I would like to give my thanks to all the people of CeDInt, I remember all the help that you have given me, six years have been quite a long time, or better to say, eight years, since my first time here. Thanks for Dani, Antonio, Regino, Guiller, Roc\{'i \}o, Carmen, Marina, Pablo Zamora, David Sanz, Eneko, Kike, Dustin, Edu, Belen, Leticia, Fran, Fran, David Garrido, Alberto, Javi, Elena, Jaime, Jorge Martin, Jesus, Cesar, Cris, Gonzalo, Dani, Luca, Ricardo, Edu, Jorge Olloqui, Silvia, Hector, Dani, David, Giulio, Igor, Guillermo Cañada, Ricardo Cañete, Jorge Gorospe, Mercedes, Olga, Pedro, Elisa, Paloma, Pilar, Milena, Bharat, Jesus, Gonzalo, Clara, Edgar, Angel, Mercelo ...

Third, I would like to thank my colleagues at ETH Zurich, it has been a pleasure working with you guys, to Friedemann, Barbara, Vincent, Mihai, Anwar, Hossein, Gabor, Leyna, Subho, Aurelia and Matthias for all your help.

Many thanks to Angel Alvarez, who provided me the first opportunity to come to UPM as exchange student and all the help during my stay here.

And to my Chinese friends, Qi, Jiayao, Wang lin, Xiaohan, Ganggao, Zhi, Cheng, Peggy, Anne, Yu, Xing, Shuo, Panpan, Jingyi, Jialin....

Last but not least, I would like to give my special thanks to my family for their support and respect all the time.

The space here is limited and I can not write down all the names of the people that have helped me in some parts of my life. I always feel that the word "thanks" is not enough to express my appreciate to the people, but I want to use this opportunity to show my appreciate 
here from my heart to all of you that have ever appeared in my life and I will never never forget. 


\begin{abstract}
We are living in a world full of embedded systems, which have formed an essential part of our daily lives. With the development of the Internet of Things, more and more Things such as sensors, actuators and smart devices are connected to collect and exchange data. However, the use of different communication protocols leads to high cost and long development time for the integration of different technologies in the same system. As a possible solution, the concept "Web of Things" (WoT) emerges with the idea of using Web as the application-layer for the IoT, drawing the attention of several researchers.

One of the issues that the IoT is facing is "data silos", i.e the isolation of data. This issue appears because the meaning of the data is not clear explained. The Web of Things dedicates to improve the semantic interoperability of the IoT, which requires the simultaneous transmission of the meaning with the data, i.e. semantics.

This thesis focus on the implementation of a semantic data management system for an open IoT platform. The platform dedicates to solve the integration problem among different communication technologies: capillary networks and traditional home automation protocols. Besides, the platform defines a common vocabulary for providing semantic description to Things, including the functionalities, representation of the generated data and related concepts such as location or user authorization. In addition, the platform implements a Web API, which allows users or software programs to interact with Things through a standard Web-based interface.

On the other hand, the involvement with real devices into the Web requires higher level of security and privacy protection, and the "multi-client, multi-user" paradigm demands more precise approach for deciding the authority of each user. To face this challenge, a new concept "Private Knowledge Graph" (PKG) has been proposed which represents a knowledge graph generated for the use of a specific agent. Besides, a fine-grained access control mechanism is designed on the basis Web Services and PKGs.

Finally, a theoretical case study is presented to explain the working process of the semantic data management system. Furthermore, a practical case is provided to validate the performance of the designed system, verifying that it facilitates the integration of Things and applications.
\end{abstract}





\section{Resumen}

Vivimos en un mundo lleno de sistemas embebidos que forman una parte esencial de nuestra vida diaria. Con el desarrollo del Internet de las Cosas (IoT), cada vez más "Cosas" como sensores, actuadores y dispositivos inteligentes están conectados para recopilar e intercambiar datos. Sin embargo, la utilización de diferentes protocolos de comunicaciones conlleva un alto coste y elevados tiempos de desarrollo para la integración de diferentes tecnologías en un mismo sistema. Como posible solución, surge el concepto de "Web de las Cosas" (WoT), con la idea de utilizar la Web como una capa de aplicación para los sistemas IoT, conviertiendose en un relevante campo de investigación.

Uno de los problemas a los que se enfrenta el IoT son los "data silos", es decir, el aislamiento de los datos. Este problema aparece porque el significado de los datos no se encentra explicado de forma clara. La Web de las Cosas busca mejorar la interoperabilidad semántica del IoT, lo que requiere la transmisión simulatánea de los datos y sus significado, es decir, su semántica.

El objetivo de esta tesis es la implementación de un sistema de gestión semántica de datos en una plataforma IoT abierta. La plataforma se dedica a solucionar el problema de integración entre diferentes tecnologías de comunicación: redes capilares y protocolos domóticos tradicionales. Además, la plataforma define un vocabulario común para proveer de una descripción semántica a las Cosas, incluyendo sus funcionalidades, la representación de los datos generados y conceptos asociados como localización o autorización del usuario. Aparte, la plataforma implementa una API Web que permite a los usuarios o programas software interactuar con las Cosas a través de un interfaz Web estándar.

Por otro lado, la implicación de dispositivos reales con la Web requiere altos niveles de seguridad y de protección de la privacidad, y el paradigma "multi-cliente, multi-usuario" hace necesaria una estrategia más precisa para decidir la autorización de cada usuario. Para resolver este problema, se ha propuesto un nuevo concepto, llamado "Grafo de Conocimiento Privado" (PKG), que representa un grafo de los conocimientos generados por el uso de un agenteespecífico. Además, se ha diseñado un mecanismo de control de acceso con alta resolución basado en los servicios Web y PKG. 
Finalmente, para explicar el funcionamiento del sistema de gestión semá ntica de datos se describe un caso de estudio teórico. Además, para su validación, se presenta un caso práctico de la implementación del sistema diseñado, demostrando que facilita la integración de Cosas y apliaciones. 


\section{Table of contents}

$\begin{array}{ll}\text { List of figures } & \text { xix }\end{array}$

$\begin{array}{ll}\text { List of tables } & \text { xxiii }\end{array}$

1 Introduction 1

1.1 Context of the thesis $\ldots \ldots \ldots \ldots \ldots$

1.1.1 Semantic interoperability . . . . . . . . . . . 2

1.1.2 Communication through standard Web technologies . . . . . . . 4

1.1.3 Fine-grained access control mechanism ........... 5

1.2 Hypothesis ....................... 5

1.3 Objectives of the thesis .................... 6

1.4 Thesis Outline . . . . . . . . . . . . . . . 6

2 State of the art 9

2.1 From the Internet of Things to the Web of Things . . . . . . . . . . 9

2.1.1 Communication protocols for Internet . . . . . . . . . . . 10

2.1 .2 Common data format . . . . . . . . . . . . . . . . . 10

2.1 .3 Web services ..................... 11

2.1 .4 WebSocket ....................... 13

2.2 Semantic web and Semantic web technologies . . . . . . . . . . . . 13

2.2.1 Clarification of terminologies . . . . . . . . . . . . . 14

2.2.2 Semantic Web Stack . . . . . . . . . . . . . . . . . . 15

2.2 .3 Java RDF API . . . . . . . . . . . . . . . . . 22

2.2 .4 Storage of ontologies . . . . . . . . . . . . . 23

2.2 .5 Inference and rules . . . . . . . . . . . . . 23

2.3 Popular knowledge bases . . . . . . . . . . . . . . . 23

2.4 Ontologies for the domain of IoT and WoT . . . . . . . . . . . . . 24

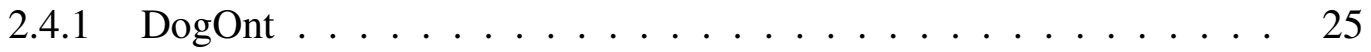


2.4.2 SSN ontology and IoT-lite . . . . . . . . . . . . 25

2.4 .3 WoT ontology . . . . . . . . . . . . . 26

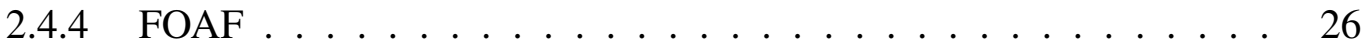

2.5 Authentication and authorization protocols . . . . . . . . . . 26

3 Background and preliminary work 29

3.1 Background . . . . . . . . . . . . . . . . . . 29

3.1.1 Wireless Transducer Network (WTN) architecture . . . . . . . 30

3.1.2 Wireless Transducer Network (WTN) devices . . . . . . . . . . . 32

3.1.3 Software platform for IoT control . . . . . . . . . . . . . 33

3.1.4 Applications for the developed IoT solution . . . . . . . . . . . 37

3.2 Parameter-based Mechanism . . . . . . . . . . . . . . . . . . . . . 39

3.2.1 Definition of parameter . . . . . . . . . . . . . . 40

3.2.2 Structure of Parameter . . . . . . . . . . . . . . . . . . . 40

3.2.3 Parameter in Central Server . . . . . . . . . . . . . . . . 44

3.2.4 Parameter in Border Router . . . . . . . . . . . . . . . . . 46

3.3 Interaction with Data Warehouse . . . . . . . . . . . . . . . . . . 49

3.3 .1 Data Warehouse . . . . . . . . . . . . . . . . 50

3.3 .2 Data Access Objects (DAOs) . . . . . . . . . . . . . . . 51

3.3.3 Creation of new annotations . . . . . . . . . . . . 53

3.3 .4 Data persistence and retrieval . . . . . . . . . . . . . . 58

3.3.5 Control of Data Consistency by DAOs . . . . . . . . . . . . . 58

3.3.6 Example Class . . . . . . . . . . . . . . . . . 59

4 System KG and Inference

4.1 Overview of the System KG . . . . . . . . . . . . . . . . . . . . 61

4.1 Creation of BATOnt . . . . . . . . . . . 63

4.1.2 Dataset structure of System KG . . . . . . . . . . . . . . 74

4.1 .3 SPARQL queries . . . . . . . . . . . . . . . 75

4.2 Inference and rules definition . . . . . . . . . . . . . 78

4.2 .1 Objective . . . . . . . . . . . . . . . . 78

4.2.2 Rule set definition . . . . . . . . . . . . . . . . 78

4.2.3 Materialized Ontology Views for System KG . . . . . . . . . 88

$5 \quad$ Fine-grained access control for the Web of Things 89

5.1 Authentication and Authorization . . . . . . . . . . . . . . . . 89

5.2 Design of a Web API . . . . . . . . . . . . . . . . . . 90 
5.2.1 Request Definition . . . . . . . . . . . . . . . . 99 91

5.2.2 Definition of the representation of resources . . . . . . . . . . 92

5.2.3 Templates for request construction . . . . . . . . . . . . 96

5.3 Security Manager Composition . . . . . . . . . . . . . . . . . . . . 98

5.4 Request Analysis . . . . . . . . . . . . . . . . . . . . . . . 100

5.4.1 Request from Border Router . . . . . . . . . . . . . . . . 102

5.4 .2 Request from user . . . . . . . . . . . . . . . . 103

5.4 .3 Security consideration . . . . . . . . . . . . . . 108

5.5 PKG management . . . . . . . . . . . . . . . . . . . . . . . . . . . 109

5.5.1 Generation and storage . . . . . . . . . . . . . . . . 109

5.5.2 Control of expiration of tokens . . . . . . . . . . . . . . . 114

5.5.3 On-the-fly updates of PKGs . . . . . . . . . . . . . 115

6 Case study and test bed $\quad 117$

6.1 Case study . . . . . . . . . . . . . . . . . . . . . . . 117

6.1 .1 Overview of KG for the client . . . . . . . . . . . . . 118

6.1 .2 Perform inference with fact rules . . . . . . . . . . . . 123

6.1.3 Perform inference with policy rules and generate PKGs . . . . . . 125

6.2 Test bed . . . . . . . . . . . . . . . . . . . . 133

6.2 .1 Overview of facilities . . . . . . . . . . . . . 133

6.2.2 Test of Web API with REST Client . . . . . . . . . . . . . . . . 139

6.2.3 Web-based user interface . . . . . . . . . . . . . . . 151

6.2.4 External application based user interface . . . . . . . . . . 163

7 Conclusion and future work $\quad \mathbf{1 7 5}$

7.1 Conclusion . . . . . . . . . . . . . . . . . . 175

7.2 Discussion and future work . . . . . . . . . . . . . . 176

7.2.1 Integration with other semantic vocabularies . . . . . . . . . 177

7.2.2 Integration with other IoT platform . . . . . . . . . . . 177

7.3 Publications . . . . . . . . . . . . . . . . . . . 177

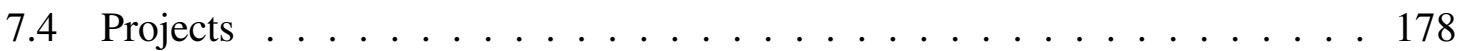

$\begin{array}{ll}\text { References } & 181\end{array}$

$\begin{array}{llr}\text { Appendix A Enumerations in BATOnt } & 189\end{array}$ 



\section{List of figures}

1.1 Use case for using semantic description . . . . . . . . . . . . . 3

1.2 Use Web as the application layer for Things . . . . . . . . . . . . 4

2.1 Semantic Web Stack . . . . . . . . . . . . . . . . . 16

3.1 System communication protocol stack . . . . . . . . . . . . 31

3.2 WTN devices . . . . . . . . . . . . . . . . . . . 32

3.3 Software architecture of Central Server . . . . . . . . . . . . . . . . . 34

3.4 Software architecture of Border Router . . . . . . . . . . . . . . . . . 36

3.5 Summary of the IoT projects . . . . . . . . . . . . . . 38

3.6 Class UML diagram of parameter and the related classes . . . . . . . . . 41

3.7 Use of parameters among the components in Central Server . . . . . . . . . 44

3.8 Negotiation through Negotiator in Central Server . . . . . . . . . . . . . 45

3.9 Use of parameter among the components in Border Router . . . . . . . . . 46

3.10 Negotiation through Negotiator in Border Router . . . . . . . . . . . . . 48

3.11 Generic DAO and DAO structure . . . . . . . . . . . . . . 52

3.12 Annotation set created in the platform . . . . . . . . . . . 55

3.13 Ontology storage and relational database schema . . . . . . . . . . 56

3.14 Java class User annotated with the created annotations $\ldots \ldots$. . . . . . . 59

4.1 Workflow of Private Knowledge Graph (PKG) generation . . . . . . . . . . 62

4.2 General view of BATOnt main classes and properties . . . . . . . . . . . 65

4.3 Class and properties in the Things perspective . . . . . . . . . . . 66

4.4 Class and properties in the control perspective . . . . . . . . . . . . . . 69

4.5 Class and properties in the spatial perspective . . . . . . . . . . . 70

4.6 Class and properties in the user perspective . . . . . . . . . . . . 72

4.7 Data Structure of System KG . . . . . . . . . . . . . . . . . 76

4.8 Subset of BATOnt . . . . . . . . . . . . . . . . . . 79

4.9 Subset of BATOnt with inferred information . . . . . . . . . . 80 
5.1 Composition of the Security Manager . . . . . . . . . . . . . . 100

5.2 Workflow of request analysis . . . . . . . . . . . . . . . . . . . 101

5.3 Workflow of request analysis for the requests from Border Routers . . . . . 102

5.4 Workflow of request analysis for a login request . . . . . . . . . . . . . . 104

5.5 Workflow of request analysis for the Logout request . . . . . . . . . . . . . 105

5.6 Workflow of request analysis for the query or modification request . . . . . 107

5.7 Description of relationships among entities in the given example . . . . . . 109

6.1 Overview of Original $\mathrm{KG}$ for client0001 . . . . . . . . . . . . . . . . 119

6.2 Statements about administration in OWL file . . . . . . . . . . . . . 120

6.3 Statements about Things in OWL file . . . . . . . . . . . . . . . . . . 121

6.4 Statements about building layout in OWL file . . . . . . . . . . . . 122

6.5 Statements about trust policies in OWL file . . . . . . . . . . . . . . 123

6.7 Statements obtained by applying fact rules in OWL file . . . . . . . . . . 123

6.6 Inference KG obtained after performing inference with fact rules . . . . . . 124

6.8 User1 related deductions obtained by performing inference with policy rules 126

6.9 Statements related to ex:RoleSW obtained by applying policy rules in OWL file 127

6.10 Statements related to ex:User1 obtained by applying policy rules in OWL file 127

6.11 User2 related deductions obtained by performing inference with policy rules 128

6.12 Statements related to ex:RoleHW obtained by applying policy rules in OWL file . . . . . . . . . . . . . . . . . . . . . . 129

6.13 Statements related to ex:User2 obtained by applying policy rules in OWL file 130

6.15 Statements related to ex:User3 obtained by applying policy rules in OWL file 130

6.14 User3 related deductions obtained by performing inference with policy rules 131

6.16 PKG-User3 in OWL file . . . . . . . . . . . . . . . . . . . . . 132

6.17 View of showroom in CeDInt . . . . . . . . . . . . . . . . 134

6.18 Demonstrator test bed . . . . . . . . . . . . . . . . . . . . . . 138

6.19 Postman page when unauthorized access happens. . . . . . . . . . . . . 139

6.20 Postman response to request login action for Johnny . . . . . . . . . . . . . 140

6.21 Send request by Postman to perform login action for GreenlabAdmin . . . . 141

6.22 Postman response to request get all user for Johnny . . . . . . . . . . . . 142

6.23 Postman response to request get all user for user GreenlabAdmin . . . . . . 143

6.24 Postman response to request get spaces for Johnny . . . . . . . . . . . 144

6.25 Send request by Postman to get all the spaces for non-administrator user . . 144

6.26 Postman response to request get all the devices for Johnny . . . . . . . . . 145

6.27 Postman response to request get all the devices for GreenlabAdmin . . . . . 146

6.28 Postman response to request get a specified device . . . . . . . . . . . . . 147 
6.29 Postman response to request get device BSN01-0042 for Johnny . . . . . . 148

6.30 Postman response to request get device BSN01-0042 for GreenlabAdmin . 148

6.31 Postman response to request get all the parameters for Johnny . . . . . . 149

6.32 Postman response to request get all the parameters for GreenlabAdmin . . . 150

6.33 Postman response to change the value of a parameter as an administrator . . 150

6.34 Postman response to change the value of a parameter with Read permission 151

6.35 Control panel of role management . . . . . . . . . . . . . . . . 152

6.36 Control panel of user creation/modification . . . . . . . . . . . . 153

6.37 Control panel of user management for Johnny . . . . . . . . . . . . 153

6.38 Control panel of space management for Johnny . . . . . . . . . . . . . . . 154

6.39 Control panel of device management for Johnny . . . . . . . . . . . . . . 154

6.40 Control panel of user management for GreenlabAdmin . . . . . . . . . . 155

6.41 Control panel of space management for GreenlabAdmin . . . . . . . . . . 155

6.42 Control panel of device management for GreenlabAdmin . . . . . . . . . . 155

6.43 Control panel of device configuration for GreenlabAdmin . . . . . . . . . . 156

6.44 Control panel of device configuration for BATSense . . . . . . . . . . . 158

6.45 Control panel for requesting parameter value in real time . . . . . . . . . . 159

6.46 Control panel of device configuration for LightActuator . . . . . . . . . . . 160

6.47 Control panel for modifying parameter value . . . . . . . . . . . . . . . 161

6.48 Control panel of historical measurements . . . . . . . . . . . . . 162

6.49 User interface of application Greenlab for Mini Greenhouse . . . . . . . . 163

6.50 3D-model based user interface for showroom control implemented in Unity 164

6.51 Workflow of the integration with 3D-model based user interface . . . . . . 165

6.52 Workflow of integration of WeChat Admin Platform with the platform . . . 167

6.53 Example WeChat message and format . . . . . . . . . . . . . . . 168

6.54 Control panel for official account setting in WeChat Admin Platform . . . . 169

6.55 Control panel for server configuration in WeChat Admin Platform . . . . . 170

6.56 Subscribe to the official account CoolHouse . . . . . . . . . . . . . . . 171

6.57 Talk with the official account CoolHouse for controlling devices in test bed 172

6.58 WeChat message sent from CoolHouse Server to WeChat Server . . . . . . 173 



\section{List of tables}

2.1 Namespaces by convension . . . . . . . . . . . . . . . . 17

4.1 Rules for deciding permissions of roles to parameters . . . . . . . . . 83

4.2 Rules for deciding permissions of users to parameters . . . . . . . . . 86

5.1 Templates for constructing requests $\ldots \ldots \ldots 7$

6.1 Definition of namespaces . . . . . . . . . . . . . . . . 117

6.2 Devices used in the test bed . . . . . . . . . . . . . . . . 135

6.3 Parameters of devices in CEDINT Space . . . . . . . . . . . . . . . 136

6.4 Parameters of devices in MiniGreenhouse Space . . . . . . . . . . . 136

6.5 Parameters of device Weather Station CEDINT . . . . . . . . . . . . 136 



\section{Chapter 1}

\section{Introduction}

Along with the fast development of the Internet of Things (IoT), more and more smart devices, including sensors, actuators and vehicles are connected to collect and exchange data. The Internet of Things allows objects to be sensed and controlled remotely. To fulfil the network connectivity, amounts of communication protocols or standards have been developed. The most popular ones are Bluetooth, Wi-Fi, Ethernet and cellular networks, such as 4G/5G, LTE. Meanwhile, some domains have their own specific protocols. For the domain of Home Automation, the most popular protocols are X10[1], KNX[2], LonWorks[3], 6LoWPAN[4], Bluetooth LE (BLE)[5], ZigBee [6] and Z-Wave[7]. The development of a smart control system may need to combine different protocols. Normally, different protocols lead to different data format and interfaces for interactions. Therefore, a mesh-up of networks with different protocols means a tough task for integration, which also results in high cost and long development time in practice. On basis of the situation mentioned above, in 2007, the idea of the Web as an application-layer for the IoT started to emerge as a new topic "the Web of Things" (WoT) and several researchers began working on this topic.

The Internet is a networking infrastructure, i.e. a massive network of networks, which connects the computers and smart objects together, where any of them can communicate with the others. The information is transmitted via a variety of protocols, which defines the data format and rules for the transmission. Accordingly, the Internet of Things focus on "get Things connected ". Whereas the Web is an abbreviation of the World Wide Web, which is an information-sharing model that is built on top of the Internet. Web uses HTTP protocol to carry out the transmission of data. The Web is used to share information, link web resources and realize the cooperation among different applications. Hence, the Web of Things focus on the semantic interoperability of Things and the collaboration among them. As a matter of fact, the WoT works as a refinement of the Internet of Things. 
The WoT focus on constructing a web of the smart objects where their data can be exchanged together with their meaning so as that the data from different Things can be integrated and shared in a semantic way. Meanwhile, by providing standard Web-based interfaces, the Things can provide services at the level of Web for the use of other Things and web-based applications.

\subsection{Context of the thesis}

Considering the ever increasing number of devices (Things) and the amount of data produced, it is demanded to facilitate the exchange of data in an "understandable" way. In order to achieve this goal, it is necessary to develop a data management system which combines the following aspects:

- Semantic interoperability, which involves the use of Semantic Web technologies for describing the data of the Things to encourage the data modelling, data sharing and data publishing.

- Communication through standard Web technologies, which involves the usage of standard Web Protocols for the communication with Things regardless their own technologies.

- Fine-grained access control mechanism, the issue about authentication and authorization for the access to the Things has also be concerned, which is to ensure the security and privacy in an IoT system.

\subsubsection{Semantic interoperability}

One of the issues that the IoT is facing is "data silos", i.e the isolation of data. This issue appears because the meaning of the data is not clear explained. The Web of Things dedicates to improve the semantic interoperability of the IoT, which requires the simultaneous transmission of the meaning with the data, i.e. semantics. This process is accomplished by adding meta-data (data about the data) and linking the data to a shared vocabulary. The meaning of the data (semantic data) is transmitted together with the data itself in the same "information package". The semantic data about a Things can include:

- Data about the description of the measurement, including the magnitude, the data type, the property that it represents, the unit and the symbol of the unit. It only make sense to compare two measurement when they are representing the same property in the same scope. 
- The functionality, the access type and the configuration of the Things.

- The location of the Things, the device or uncontrollable object associated, and even the user who is using the Things.

By adding semantic description to the Things, the integration of different data resources can be realized. Fig. 1.1 gives an example for illustrating the use of semantic data for the Things.

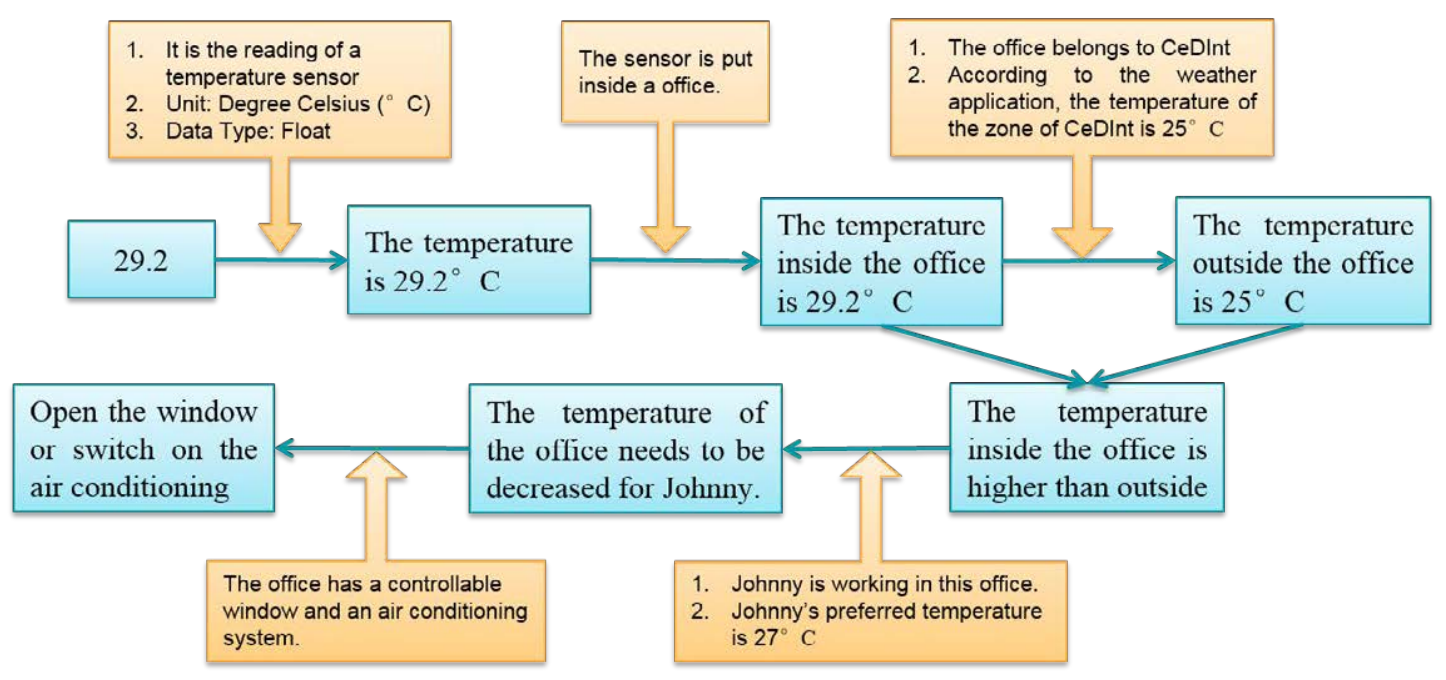

Fig. 1.1 Use case for using semantic description

As illustrated, by knowing about "29.2",there is no any information about what it represents. By knowing the semantic data, the meaning of this string can be obtained:

- Knowing that this string is the reading of a temperature sensor, it can be known that it represent the temperature is 29.2 degrees

- Knowing that the sensor is inside a office of CeDInt, and according to the data from the weather application saying that the local temperature is 25 degrees, it can be inferred that the temperature inside the office is higher than outside

- By knowing that Johnny is currently in the office and his preferred temperature is 27 degrees, it can be inferred that the temperature should be decreased for Johnny

- By knowing that the office has a controllable window and an air conditioning system, the corresponding actions can be executed, either open the window or switch on the air conditioning. 


\subsubsection{Communication through standard Web technologies}

By using Web as the application layer of the Internet, the Things can be accessed or controlled through standard Web technologies regardless the communication protocols they use. While the IoT protocols are used to get the Things connected, the Web protocols can help them to expose their services to the others, including users, web-applications, REST client tools and other IoT platform. In this way, the users and other computers are able to communicate with the developed IoT in a standard way. This will also encourage the integration with different types of user interfaces such as chat application, 3D-Model based interface, as shown in Fig. 1.2

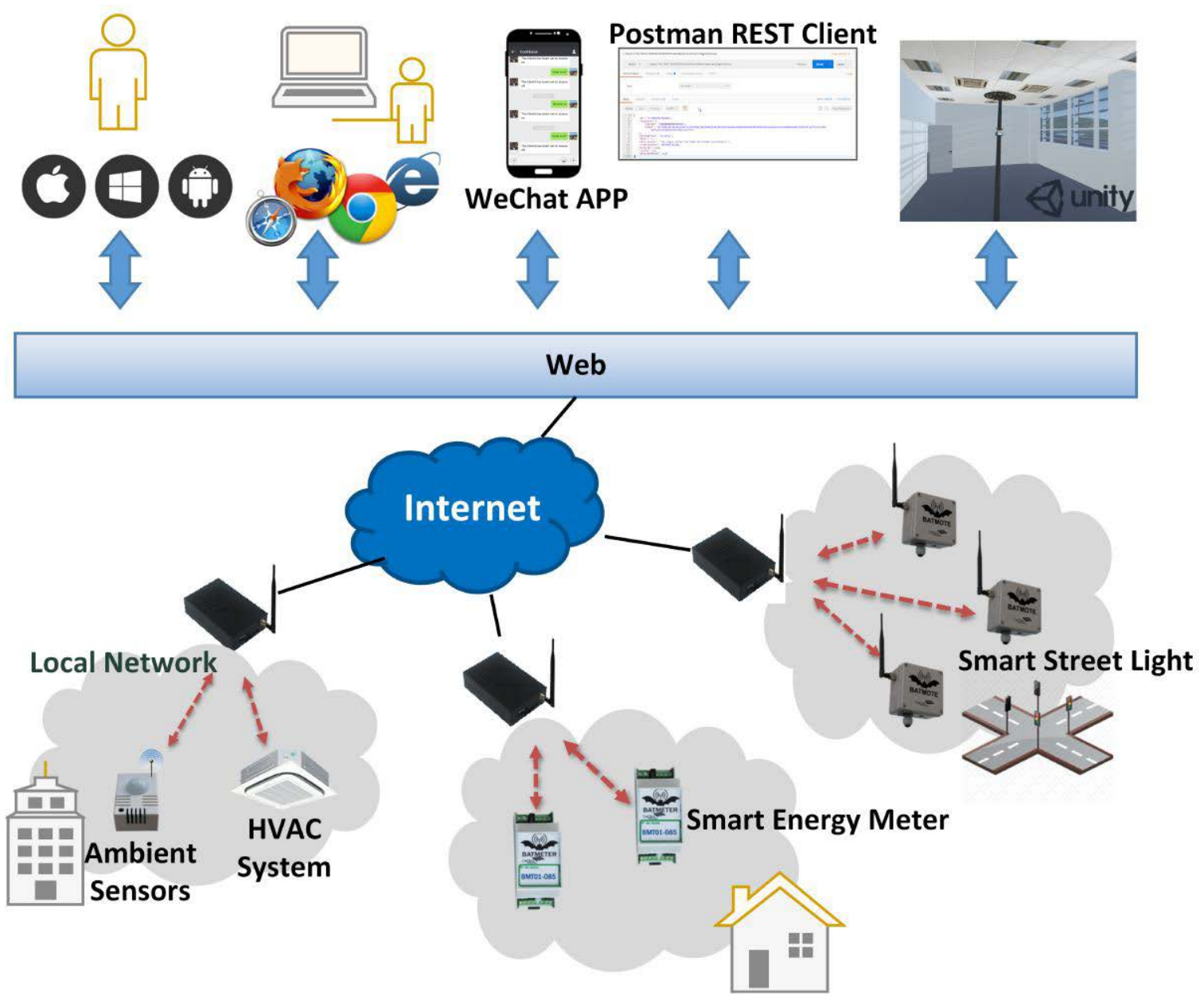

Fig. 1.2 Use Web as the application layer for Things

As illustrated, different Things can be used for the projects of different purposes, including smart home, smart building and smart city. Each of them communicate in their local network with their own technologies and get connected to the Internet through the border router. By 
interacting through the standard Web-based interface, the users or computers can access and control the Things without concerning about the technologies and the communication protocols that have been used in the local network. Instead, they can only focus on the functionality that the Things provide.

\subsubsection{Fine-grained access control mechanism}

Considering the inherent characteristics of Web and the Internet of Things, how to ensure the security and privacy of both data and devices is a challenge for the research community. Providing access to Things at the level of web and application brings new challenges for the IoT solutions. The involvement of physical devices, including sensors and actuators into the Web requires higher level of security and privacy protection. Meanwhile, the involvement of large amount of users and different user groups requires more precise approach for deciding the authority of each user. Both of these requirements bring more challenges to the control of access and therefore, a fine-grained mechanism is imperative for the Web of Things.

\subsection{Hypothesis}

The hypothesis of this work is that using a data management system which combines the adaptation of a variety of Semantic Web technologies and standard Web protocols, the exchange of data can be accomplished in a semantic and secure way, allowing the fast development of cross-domain applications. In this thesis, the data management system is implemented with the following technologies:

- Ontology Engineering: a common vocabulary can be created by data modelling tools to give semantic description for the Things, including the functionality, measurements and access type. Meanwhile, the relationships among users, locations, devices, applications can be described.

- Inference: the authorization relationships between the user and Things can be obtained on the basis of a set of rules, which define the trust policies.

- Query Language: retrieving a subset of triple from the system knowledge base for the use of a user can restrict his access to the authorized Things.

- Web technologies: the user can perform the interaction with Things using standard Web-based interfaces. 


\subsection{Objectives of the thesis}

The main objective of this thesis is to design the data management of an open IoT platform, which adheres to the principles of the WoT. For achieving this objective, the work comprises the following tasks:

- Create an vocabulary for providing semantic data for the Things and their measurement. The vocabulary is for describing not only the Things, but also the related concepts that could be involved in an IoT project implementation, which are about people, spaces, control and authorization.

- Design a server-side Web API to provide the user the access to Things using standard Web protocols, regardless of their communication protocols.

- Design a fine-grained authorization mechanism for the access control to Things in a multi-client, multi-user and multi-application system

- Evaluate the theory and designed solution in a prototypical system and show the feasibility of the integration of IoT device with the existing web applications.

\subsection{Thesis Outline}

This document is organized as follows:

- Chapter 2 reviews the state of the art about the currently works that have done about the WoT, Semantic Web technologies, the recognized knowledge base, ontologies and the popular protocols for authentication and authorization.

- Chapter 3 introduces the background of the research and the preliminary work that has been done.

- Chapter 4 describes the construction and management of the system knowledge base, which includes the creation of an IoT-centric ontology BATOnt, the inference process and the definition of rules for facts and trust policies.

- Chapter 5 explains the design of a Web API for the communication with the IoT platform and a fine-grained access control mechanism that has been implemented for dealing with the authentication and authorization challenge. 
- Chapter 6 presents a case study and a test bed to assess and illustrate the feasibility of the mechanism designed and the applicability of the developed system in practical cases.

- Finally, Chapter 7 summarize the obtained results and propose possible future works. 



\section{Chapter 2}

\section{State of the art}

\subsection{From the Internet of Things to the Web of Things}

In a world where access to Internet was mainly done by people through a computer, tablet or smart phone, the concept of Internet of Things (IoT) appears as the ability to connect objects (things) to Internet. The inter-networking of physical devices (embedded systems) enables objects to collect data through sensors or actuators and exchange information. IoT concept was proposed in 1999 by Kevin Ashton. [8] Later, in 2011, the company Ericsson published a White Paper about IoT where the estimation is that more than 50 billion devices will be connected to Internet by 2020[9]. Due to the different technologies used for connecting Things to internet, there is a problem with interoperability across platforms. As a result developers are faced with data silos and high costs. In 2007, a new concept the Web of Things (WoT) started to emerge, which concerns about using Web as the application layer for the controlling of Things. The Web of Things (WoT) is a term used to describe approaches, software architectural styles and programming patterns that allow real-world objects to be part of the World Wide Web.[10]

In 2007, the Web of Things community was created by Dom Guinard and Vlad Trifa. [11] Several papers have been published, which proposed the architecture for the WoT where the Things are accessible according to the RESTful principle, which makes them part of a Web of Things. [12]. The WoT is regarded as refinement of the IoT, which bring the Things to the Web as its application layer. [13] In 2011, two PhD theses were presented at ETH Zurich on the topic of the Web of Things, which are: Building Blocks for a Participatory Web of Things: Devices, Infrastructures, and Programming Frameworks from Vlad Trifa [14] and A Web of Things Application Architecture - Integrating the Real-World into the Web from Dominique Guinard[15]. In 2014, W3C created the Web of Things Interest Group, which dedicates in developing initial standards for the Web of Things, tasked with the goal to counter the 
fragmentation of the IoT. [16] Currently, a WoT Current Practice [17] is under construction, which focuses on the task that to provide semantic data to the Things in a Thing Description so as that their capabilities and metadata can be described in a machine-understandable format.

The WoT focuses on integrating the real-world objects, i.e. the Things into the World Wide Web so as that they can be accessed from everywhere in the world. Meanwhile, it also concerns that data collected from different resources can be shared and converged in a easier way for the use of analysis, data mining and prediction. To realize these objectives, instead of inventing new technologies, the WoT adopts the standard web technologies such as Web Services and Semantic Web technologies.

The following sections will give a review about state of the art of the web services, semantic web technologies, which are used for the implementation of the Web of Things. Meanwhile, a study about the popular knowledge bases and ontologies for the domain of IoT and WoT are is given. Finally, a research about the widely applied authentication and authorization protocols will be given.

\subsubsection{Communication protocols for Internet}

This section will give a review about the common data format for exchange and the communication protocols for Internet.

\subsubsection{Common data format}

For achieving this, the standard machine-readable data formats should be used such as XML and JSON. The most widely used format of web services are XML and JSON.

XML

XML stands for Extensible Markup Language, which is a markup language for creating documents with structured data[18]. XML is used for data exchange and storage, which is designed for both human-readable and machine-readable. Listing 2.1 gives an example of XML document. 


<person>
$\quad<$ name $>$ Johnny</name $>$
$\quad<$ age $>24<$ /age $>$
$\quad<$ email $>$ johnny@example. com</email $>$
$<$ person $>$

Listing 2.1 Example of XML document

\section{JSON}

JSON stands for JavaScript Object Notation, which is a lightweight data-interchange format. A JSON object is easy for humans to read and write, and also easy for machines to parse and generate. [19] A JSON object can built with a collection of name/value pairs or an ordered list of values. Listing2.2 gives an example of JSON object.

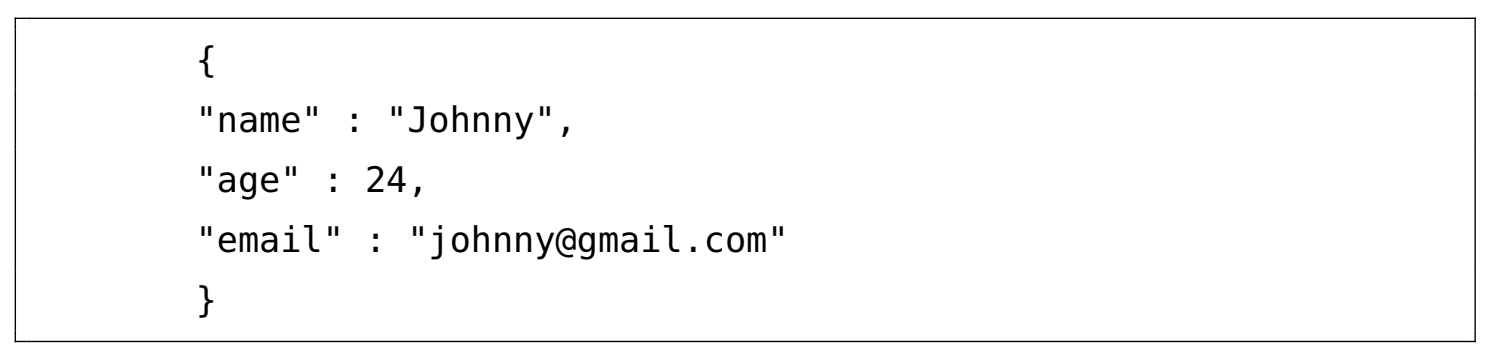

Listing 2.2 Example of JSON object

\subsubsection{Web services}

According to the definition in the W3C Web Services Glossary, a web service is "a software system designed to support interoperable machine-to-machine interaction over a network".[20] Web services enables the web technologies such as HTTP, which was originally designed for human-to machine communication to be used for machine-to-machine communication. Nowadays, the most widely adopted form of web services are Simple Object Access Protocol (SOAP) and Representational state transfer (REST).

\section{Simple Object Access Protocol(SOAP)}

Simple Object Access Protocol (SOAP) is a protocol specification for the implementation of web services. It is used for the exchange of structured information in the network of computers. The message of SOAP is represented excursively in the form of XML. SOAP was originally developed by MicroSoft [21] and became a W3C recommendation in 2003[22]. However, many developers found that SOAP is cumbersome and hard to use as even for a 
simple task, a message should be created following the required XML structure. As a result, a lighter weight alternative REST became gradually more popular.

\section{Representational state transfer (REST)}

REST stands for Representational state transfer, which is a lightweight form of Web Services comparing to SOAP [23]. Instead of using XML to make a request, REST relies on a simple URL in many cases. REST uses URIs to identify Web resources. REST or RESTful web services provides allow to access and manipulate textual representations of Web resources using a uniform and predefined set of stateless operations. The concept REST was first introduced and defined by Roy Fielding in his doctoral dissertation in 2000[24]. Since then, the word REST API or RESTful API became widely used rapidly. REST has the following advantages:

1. Stateless interactions between requests. Each request must be self-contained with all the necessary information to serve the request.

2. Independent of technologies. With REST as the interface for interactions, the changes in the underlying layer will not affect the functions provided. The changes can be about the programming language, or use of other type of database or integration of new drivers. This allows the developers to improve the underlying implementation while maintaining a stable REST API.

3. Scalability for new concepts. In REST, each resources is represented with URI and the clients are supposed to follow the links in resources to find other resources and the interactions with services. Hence, the API can easily incorporate new resources.

4. The maturity of technologies. With the fast development, today there are many implementation and frameworks to carry out RESTful Web Services. This provides a wide range of possibilities to interact with the system, including simple web sites, mobile applications, browser add-ons.

5. Good recognition in the community. From the developer's point of view, the good and comprehensive documents and friendly community of REST can help them to have a shorter learning curve of new APIs.

However, in 2008, Roy Fielding pointed out in his article "REST APIs must be hypertextdriven" that people are calling any HTTP-based interface a REST API and pointed out that: A REST API should not be dependent on any single communication protocol. It must not define fixed resource names or hierarchies. Meanwhile, the URLs should be opaque, a REST 
API should be entered with no prior knowledge beyond the initial URI. [25] As a result, most of the so called RESTful APIs today are actually HTTP-based API or Web API, which is a programmatic interface exposed via the Web, and most commonly rely on the HTTP requests. However, the word REST has been widely used due to its popularity and many developers have been calling their APIs that provide a web service over HTTP as a "RESTful API".

The REST requests can be sent by various types of terminals, which can also be used for HTTP API and Web API:

- The most common way is to make the requests from a single web page containing JavaScript. The request can be sent by using simple JavaScript or some libraries such as JQuery or AngularJS.

- The requests can be made from a software program that implements a REST Client API such as Jersey Client API [26] and unirest [27]. The program can be a mobile application or another IoT platform.

- Through plug-ins or add-ons for browsers, such as Advanced Rest Client [28] and Postman-REST Client [29] for Google Chrome, and RESTClient [30] for Firefox.

\subsubsection{WebSocket}

WebSocket is "a protocol that allows for a persistent TCP connection between server and client so they can exchange data at any time. Through WebSocket, servers can pass data to a client without prior client request, allowing for dynamic content updates". [31] The WebSocket Protocol is an independent TCP-based protocol which provideds full-duplex communication channels over a single TCP connection. The protocol was standardized by the IETF as RFC 6455 in 2011. [32] WebSocket allows to create a bidirectional ongoing conversation between a client and a server. The communications are performed over TCP port number 80 or 443 in the case of TLS-encrypted connections.

\subsection{Semantic web and Semantic web technologies}

Semantic Web is a collaborative movement among an amount of organizations to promote common format for data exchange on the World Wide Web. This collaboration is led by the World Wide Web Consortium (W3C), which is the international standards body. The objective of Semantic Web is to build a "web of data", where all the data are represented in the machine-readable form. To realize this, the semantic information should be included in the web pages so as to convert the unstructured document into structured data. 


\subsubsection{Clarification of terminologies}

In this thesis, an amount of terminologies in the domain of semantic web are used. To avoid ambiguity, some definitions of terminologies are given for clarification about Ontology, Linked Data, knowledge base and knowledge graph before the introduction of semantic web technologies.

\section{Ontology}

Ontology is one of the key concept in the Semantic Web. It is used for achieving agreement among different entities so that they can communicate and share information in the same "language". The term "ontology" comes originally from philosophy which represents the nature of being, becoming, existence or reality and was then adapted in computer science. In 1995, Tom Gruber introduced ontology as a technical term in computer science in the paper "Toward Principles for the Design of Ontologies Used for Knowledge Sharing" and defined it as a "formal, explicit specification of a conceptualization", where "a conceptualization is an abstract, simplified view of the world that we wish to represent for some purpose."[33]. In 1997, Borst pointed out in his doctoral thesis that [34] an ontology should be a conceptualization that expresses a shared view between several parties and it should be represented in a machine readable format. In 1998, Studer et al. merged the two definitions above and gave the definition that "an ontology is a formal, explicit specification of a shared conceptualization." [35]. An ontology "consists of a formal representation of a set of concepts within a domain and the relationships between those concepts" [36], which can be used to provide a semantic description of a domain. An ontology provides a schema for transforming unstructured information into structured data and ease the process of data publishing and sharing. Ontology engineering refers to the methods and methodologies for building ontologies.

\section{Linked Data}

The term "Linked Data" refers to a method of publishing structured data on the Web in a machine-readable form and can be linked with external data sets. The data it is interlinked and its meaning is explicitly defined so that it can be traversed through standard semantic queries as. [37] The main purpose of Linked Data is to use the Web to connect related data which was previously separated or isolated. The most widely used website for the Linked Data community is linkeddata.org [38] where the data set can be published and linked with other data sets. 


\section{Knowledge base and knowledge graph}

The term "knowledge base" (KB) refers to a technology used to store structured information, which was proposed in connection with expert system where structured data is needed to perform reasoning and draw conclusions.[39] At the beginning, the term Knowledge Base was only for the use of automated systems. However, as the technology scaled up, the term "Knowledge Base" is used in more and more domain and becomes ambiguous. For instance, $\mathrm{KB}$ is used in knowledge management products to describe the repositories and is used in Internet to represent Internet documents. As a result, to distinguish from the usage of term "Knowledge Base" in other systems and applications and on consideration of the graph-based form of structure in which the data is stored, the term "Knowledge Graph" $(\mathrm{KG})$ is used instead in this thesis to denote the collection of structured data. In the paper "Computing Semantic Similarity of Concepts in Knowledge Graphs" [40], the authors gave the same consideration and have given an example of KG which is constructed on the basis of WordNet. Each KG comprises a set of statements which are represented in the form of triples, i.e. subject, predict and object.

A KG is comprised of two types of axioms: terminology box (TBox) and assertion box (ABox). TBox axioms are used to describe constraints on the structure of the domain and provide a conceptual schema, while ABox axioms are used to asserts the facts. [41] The term "ontology" is often used to refer to TBox, but in OWL (Web Ontology Language), an ontology can consist of a mixture of both TBox and ABox axioms. In this thesis, to avoid ambiguity, ontology is used to denote only the TBox part .

\subsubsection{Semantic Web Stack}

Semantic web technologies refer to a series of languages and technologies for the creation of Semantic Web. In 2000, Tim Berners-Lee proposed for the first time an illustration of Semantic Web Stack in his talk about Semantic Web on XML [42]. The stack keeps evolving and Fig. 2.1[43] shows the most updated structure of the semantic web stack. 


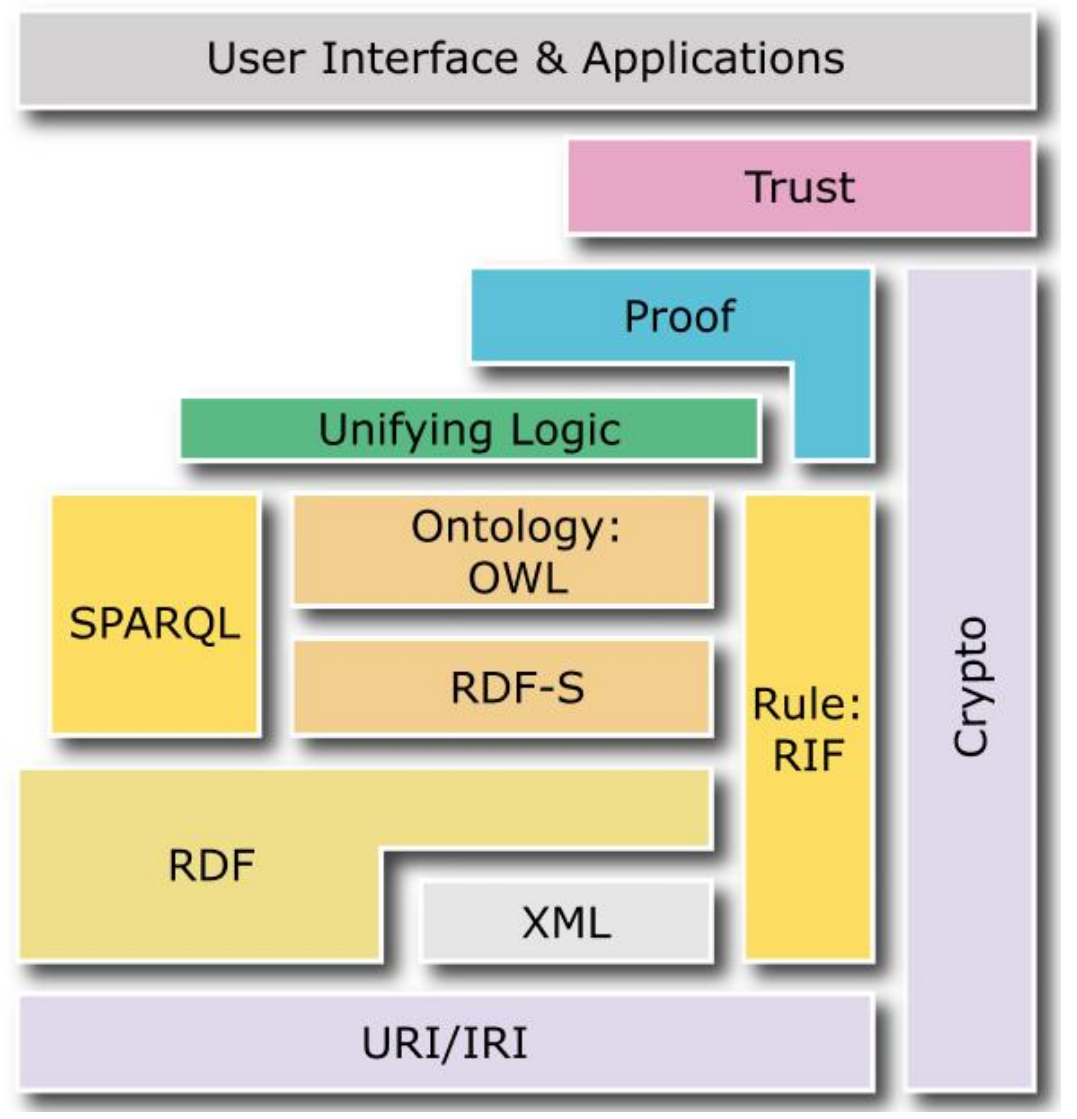

Fig. 2.1 Semantic Web Stack

The Semantic Web Stack illustrates the architecture of the Semantic Web and the technologies that can be applied. So far, the process of standardization has been done for the technologies from the bottom of the stack up to OWL, while it is still not clear that how the ones in the upper parts should be implemented. A detailed introduction will be given in the following part about the technologies RDF, RDFS, OWL and SPARQL, which are standardized by W3C, and in addition, two Semantic Web technologies Named Graph and $\mathrm{RDFa}$, which are not shown in the Semantic Web Stack view.

In the Semantic Web, the resource are denoted by URIs or IRIs. URI stands for Uniform Resource Identifier, which refers to a string that can identify a resource uniquely. The syntax of URI was first defined in 1998 by the Request for Comments (RFC) 2396 [44] and was finalized 2005 by the RFC 3986 [45]. A generic URI has the following form:

scheme:[//[user[:password]@]host[:port]][/path][?query][\#fragment]

The scheme is a string that refers to a specification for assigning identifiers within that scheme, the common scheme names include http, urn, file and ftp. The schema name is followed by two slashes (//) and then the authority part, which includes the information 
about user, password, host and port. The combination of path and two optional strings, query and fragment indicates the location of the resource that the URI indicates. IRI stands for Internationalized Resource Identifier, which is a Unicode [46] string that follows the syntax defined in RFC 3987[47]. IRIs are a generalization of URIs which permits a wider range of Unicode characters. Every absolute URI is an IRI, but not every IRI is an URI. An other common used term is URL, which stands for Uniform Resource Locator [48]. URL is used to specify the location of a web resource on a computer network and a mechanism for retrieving it. A URL is a specific type of URI.

The Semantic Web technologies are built on the well known technologies of hypertext web XML and XML Namesapce. XML namespaces are used for providing uniquely named elements and attributes in an XML document by associating them with namespaces identified by URI references[49]. Table. 2.1 shows the namespaces that have been defined by convention.

\begin{tabular}{|c||c|}
\hline \multicolumn{1}{|c||}{ Prefix } & URL \\
\hline rdf & http://www.w3.org/1999/02/22-rdf-syntax-ns\# \\
\hline rdfs & http://www.w3.org/2000/01/rdf-schema\# \\
\hline xsd & http://www.w3.org/2001/XMLSchema\# \\
\hline owl & http://www.w3.org/2002/07/owl\# \\
\hline
\end{tabular}

Table 2.1 Namespaces by convension

RDF, RDFS and OWL are all means to construct structured data models, each of which has stronger capability to express and describe the information than the former one. All of the three languages can be serialised in RDF/XML syntax.

\section{Resource Description Framework (RDF)}

Resource Description Framework (RDF) is a framework to build data model for objects ("resources") and relations between them. [50] In RDF, each resource and relationship is represented by an IRI. RDF provides the basic primitives for ontology modelling and it allows the exchange of structured data. Each statement of RDF is presented in the form of "subject-predicate-object", which is usually referred to as a "triple". A set of RDF triples is often called an "RDF graph". As a result, the database for the storage of RDF is often called a "Triple Store" or "Graph Store". Listing 2.3 shows an example of a RDF triple. 
uri://people\#Johnny http://www.example.com/knows uri://people\#Alice

Listing 2.3 Example of RDF triple

RDF can be represented in XML syntax, Listing 2.4 gives an example of RDF document written in XML syntax.

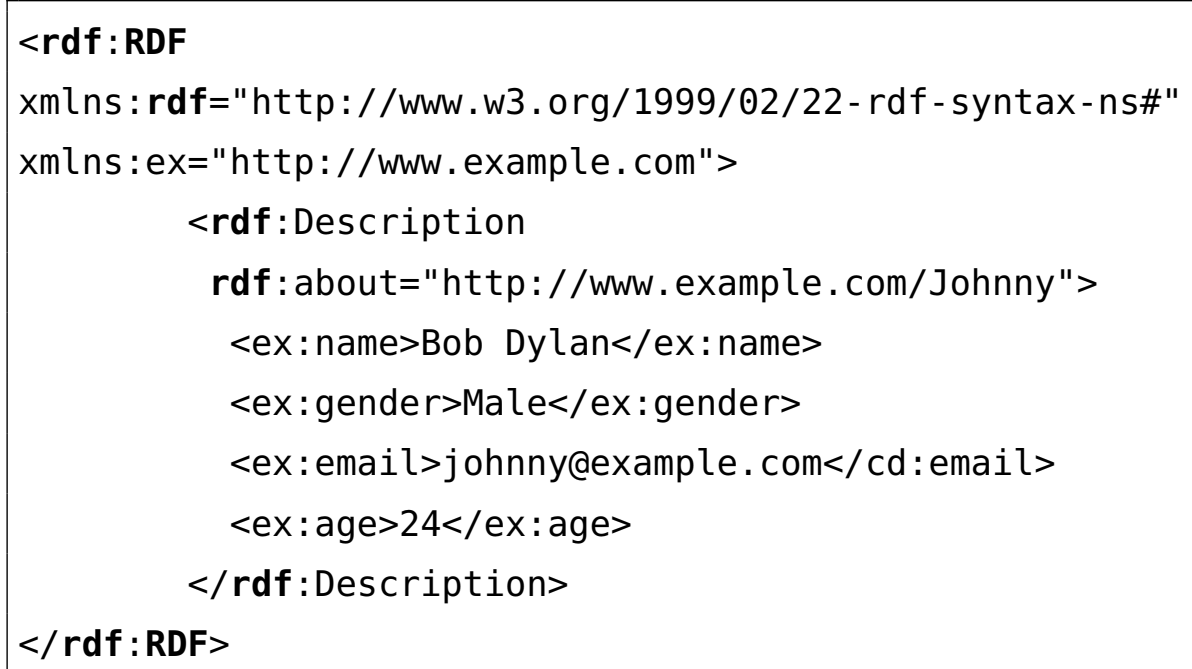

Listing 2.4 Example of RDF data model in XML syntax

\section{Resource Description Framework Schema (RDFS)}

Resource Description Framework Schema (RDFS) is an extension of the basic RDF vocabulary, which provides a data-modelling vocabulary for describing the classes and properties of RDF resources[51]. Listing 2.5 gives an example of RDFS, which defines the range of the property email as "email" and the domain as "Person". Both of rdfs:range and rdfs:domain are instances of rdf:Property. The rdfs:range is used to state that the values of a property are instances of one or more classes and rdfs:domain is used to state that any resource that has the given property is an instance of one or more classes. 


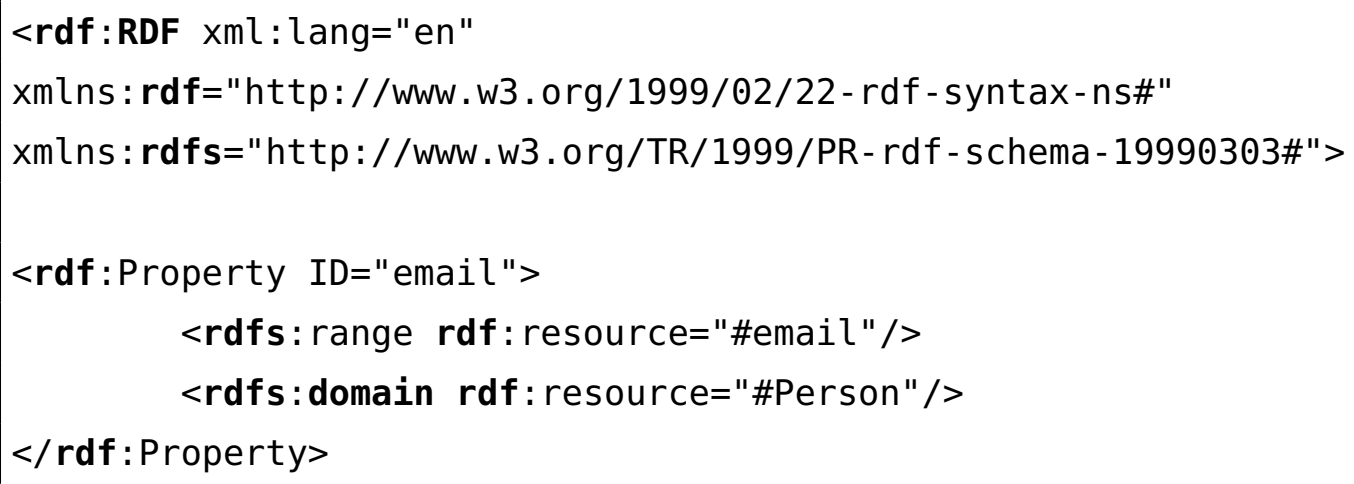

Listing 2.5 Example of RDFS

\section{Web Ontology Language (OWL)}

OWL stands for Web Ontology Language, which provides more vocabulary to describe semantics of RDF statements to facilitate greater machine interpretability of Web content. It adds more vocabulary for describing properties and classes such as cardinality (owl:maxCardinality, owl:minCardinality, owl:cardinality), equality (owl:sameAs) and enumerated classes (owl:oneOf).

OWL defines the class owl:Class which is a subclass of the rdfs:Class. In OWL, individuals are instances of the classes. [52] OWL also defines the owl:ObjectProperty and owl:DatatypeProperty as subclasses of the RDF class rdf:Property. Object properties link individuals to individuals, while datatype properties link individuals to data values. [53]. In OWL, owl:Thing is the class of all individuals.

The OWL was first published by the W3C Web Ontology Working Group in 2004. The current version of OWL, also referred to as "OWL 2" is an extension and revision of this, which was developed by the W3C OWL Working Group and published in 2009, with a Second Edition published in 2012. Listing 2.6 shows an example of enumerations in OWL for the five oceans of the world. 


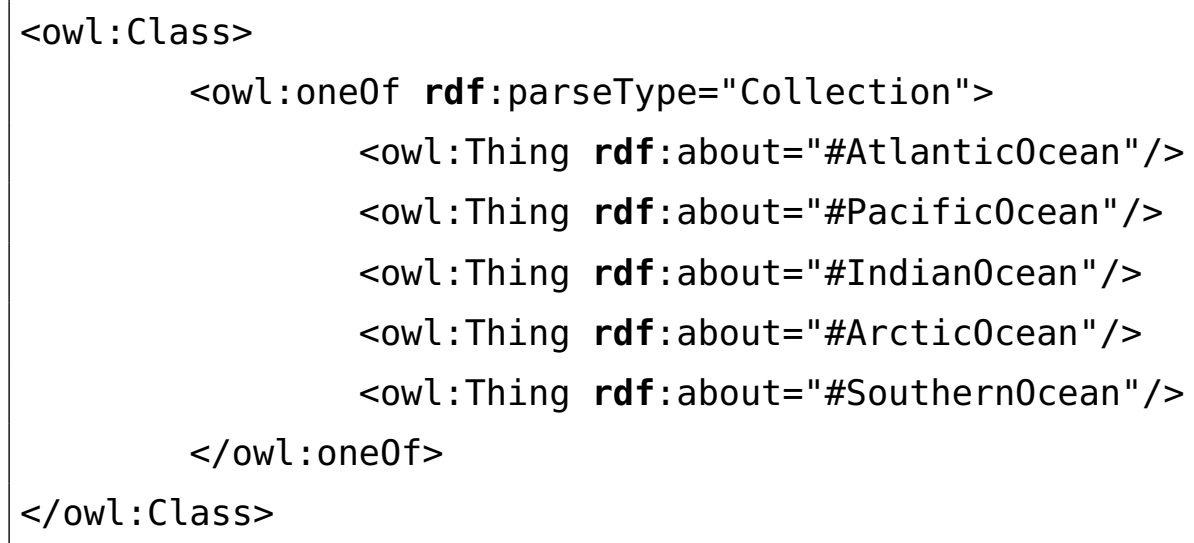

Listing 2.6 Example of OWL

\section{Named Graphs (NG)}

Named Graphs is a simple extension of the RDF data model through which graphs can be created. Each named graph consists of a set of RDF triples and is assigned a name in the form of a URI as its identifier.[54] By doing this, the set of RDF turns into quad store or a named graph.

\section{Resource Description Framework in Attributes (RDFa)}

Resource Description Framework in Attributes (RDFa) is a W3C Recommendation [55] which aims to convert the content of existing human-readable Web page into machinereadable data by embedding semantic metadata within the Web documents such as HTML, XHTML and other XML-based document. Listing 2.7 gives an example of the RDFa document. 


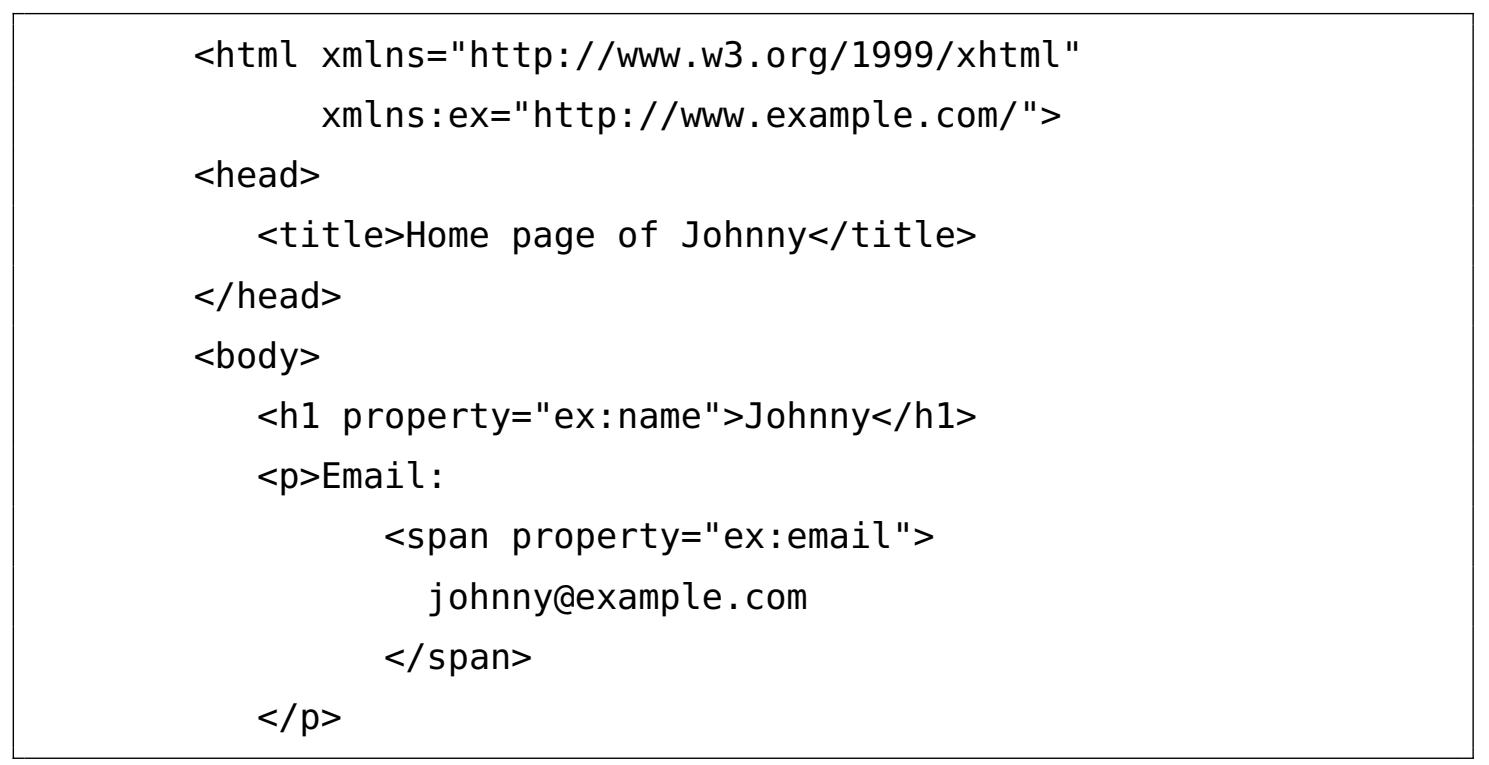

Listing 2.7 Example of RDFa

\section{SPARQL and SPARQL 1.1}

The standard query language for ontology is SPARQL, which stands for SPARQL Protocol and RDF Query Language. The SPARQL language specifies four different query variations for different purposes: SELECT query to extract raw values from a SPARQL endpoint, CONSTRUCT query to extract information from the SPARQL endpoint and transform the results into valid RDF, ASK query to provide a simple True/False result for a query on a SPARQL endpoint and DESCRIBE query to extract an RDF graph from the SPARQL endpoint. 2.8 provides an example of a SPARQL query for asking for the name of all the resources whose type is ex:Person. In the case that the RDF triples are stored in a named graph, the URI of the targeted graph should also be indicated by the keyword "FROM NAMED". Listing 2.8 provides an example of a SPARQL query. 


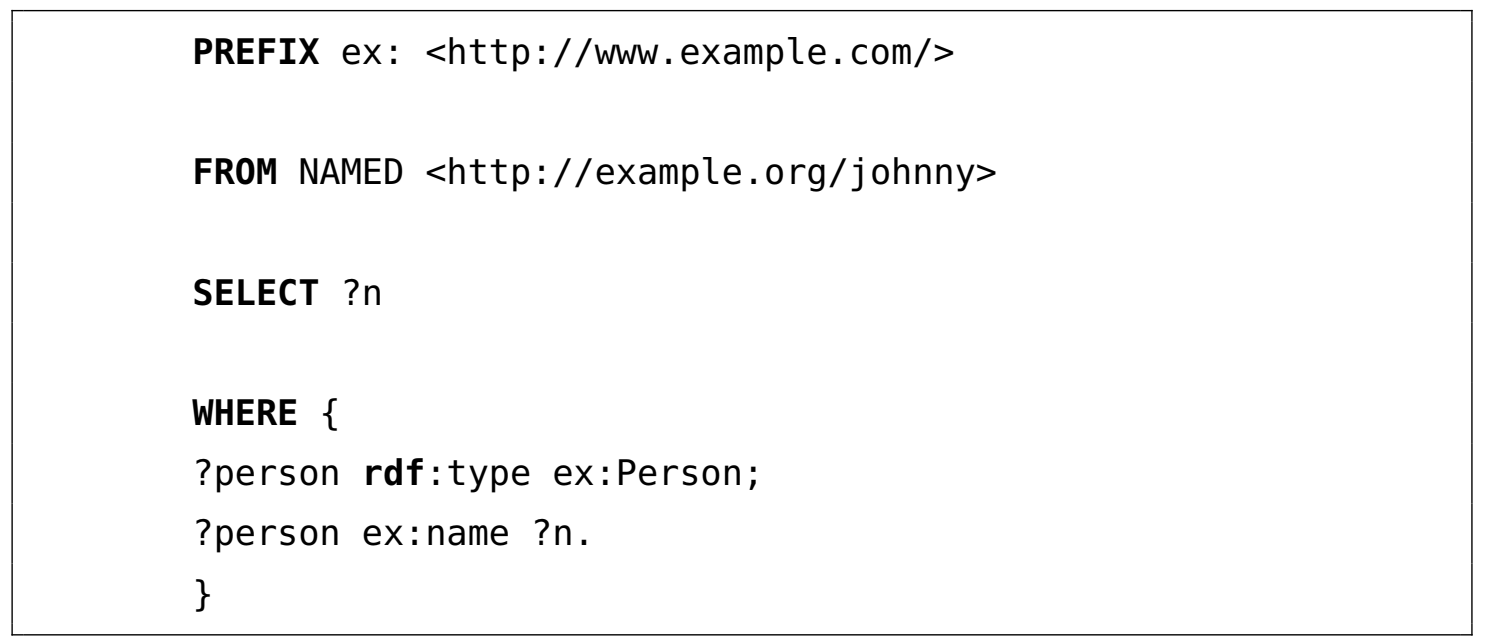

\section{Listing 2.8 Example SPARQL query}

In January, 2012, W3C publish the SPARQL 1.1 Update, which is an update language for RDF graphs. SPARQL 1.1 uses a syntax derived from the SPARQL to perform the update operations on the graphs in a Graph Store. The operations provided include update, create, and remove RDF graphs in a Graph Store. Listing 2.9 provides an example of a DELETE query which remove the RDF triples that have the individuals of ex:Person as their subjects.

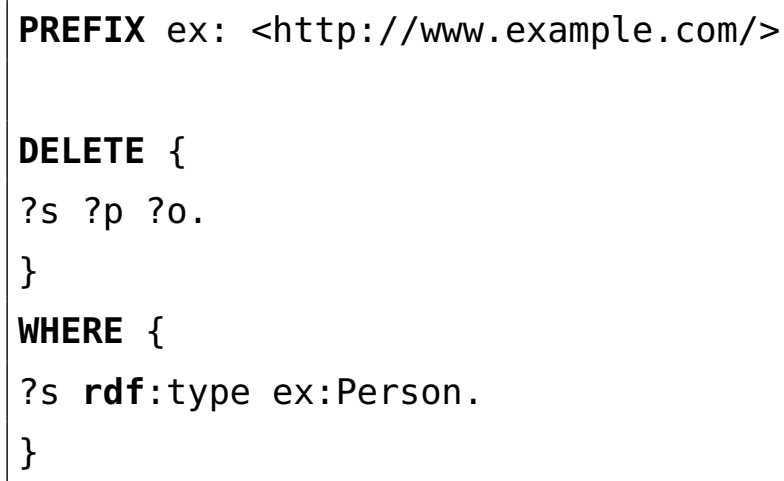

Listing 2.9 Example SPARQL 1.1 CONSTRUCT query

\subsubsection{Java RDF API}

For dealing with the RDF graphs, a Java RDF API is needed. Apache Jena is an open source Java framework for building semantic web and Linked Data applications. It provides an Java API to extract data from and write to RDF graphs. [56]. Meanwhile, the framework contains different APIs for performing different tasks of interactions with RDF data. In total, Apache Jena has six major parts: the RDF API and ARQ to query and modify the RDF graphs; TDB, a native high performance triple store to persist the data; Fuseki, a SPARQL endpoint to 
expose the datasets; Ontology API to interact with RDFS and the OWL documents and the Inference API for carrying out the inference over the data sets in the triple store.

\subsubsection{Storage of ontologies}

An ontology can be stored in a file or a triple store. There are two widely used triple stores, Jena TDB and Virtuoso. Jena TDB is a component of Apache Jena for RDF storage and query, which supports the full range of Jena APIs and can be used as a high performance RDF store on a single machine. TDB uses transactions to protect the dataset against corruption, unexpected process terminations and system crashes. TDB also provides a SPARQL endpoint for query, update and update the dataset over HTTP. [57] Virtuoso RDF Triple Store is an open source triple store provided by OpenLink Software[58], which also provides a SPARQL endpoint to query the stored datasets.

\subsubsection{Inference and rules}

In a knowledge-based system, inference is used to retrieve the implied information from the currently explicitly stated information. By using the inference, the system can achieve more intelligence. The inference is performed by a reasoner according to a set of rules, which state the logic to follow for the reasoning.

Jena provides a general purpose rule-based reasoner, which allows the users to define their own rules. This reasoner supports rule-based inference over RDF graphs and provides forward chaining, backward chaining and a hybrid execution model. [56] All the rules should be denoted with different names and can be stored in the same or separated files with the extension .rules.

\subsection{Popular knowledge bases}

Semantic Web technologies have been applied in many places and are being used in the daily life of people. There are several knowledge providers, either from the commercial companies or from academic communities. The most widely-used knowledge bases include the Knowledge Graph from Google, the Open Graph from Facebook and DBPedia. 


\section{Knowledge Graph}

The Knowledge Graph [59] is a knowledge base used by Google, which gathers information from a variety of data sources. By using the Knowledge Graph, the search engine of google is able to provide user with the information that is semantically related to their search result.

\section{Open Graph protocol}

The Open Graph protocol is initialized by Facebook [60], which enables any web page to become a rich object in a social graph. [61] The Open Graph protocol is implemented by embedding RDFa data with the namespace "og: http://ogp.me/ns" into the web pages to mark up the attributes of objects. The attributes include the basic metadata og:title, og:type, og:image, og:url and the optional metadata og:audio, og:description, og:determiner, og:locale, og:locale:alternate, og:site_name, og:video. [62]

\section{DBpedia}

DBpedia [63] is a research project which aims to extract structured content from the information in Wikipedia and makes it available on the World Wide Web. DBpedia allows users to semantically query relationships and properties of Wikipedia resources. A public SPARQL endpoint is provided by DBpedia at http://dbpedia.org/sparql [64] for the online access to the data sets of the DBpedia.

The above knowledge bases work together and form the Web of Data, which eases the process and improve the experience for users to obtain information. However, all of these projects stay in the level of virtual world without the involvement of real-time interaction with the physical objects. The WoT will be able to extend the currently Web of Data to the physical world.

\subsection{Ontologies for the domain of IoT and WoT}

For the domain of the IoT and the WoT, some ontologies have been created. The most widely recognized ones are DOGOnt and the Semantic Sensor Network (SSN), and an ontology for describing the domain of the WoT has recently been developed. This will also do a review about the popular ontology FOAF which is used for linking people and information. 


\subsubsection{DogOnt}

The first recognized ontology for the domain of IoT is DogOnt, which was developed in 2008 by the e-lite group from the University Politecnico di Torino. DogOnt is a modeling language for IDEs (Intelligent Domotic Environment) [65], which is able to describe the following concepts:

[1] The capabilities of domotic devices

[2] The technology specification of the interaction interface of the device

[3] The possible configuration of the devices

[4] The composition of the home environment

[5] The layout inside a home and the location of devices

DogOnt give a good demonstration about the construction of an ontology for the domotic devices. However, DogOnt is only restricted to describe the situation in a home environment. Moreover, with the development of IoT technologies, more new type of devices and communication protocols are developed, DogOnt is not capable to describe the new invented devices and has since been outdated.

On the basis of DogOnt, a gateway Dog Gateway has been implemented based on it. Dog Gateway is still under development. It targets at a single home automation technology at the first [66] and has been nowadays an open source, OSGi compliant implementation of smart environment gateway and IoT platform featuring multi-protocol capabilities, REST-based application APIs. [67][68][69]

\subsubsection{SSN ontology and IoT-lite}

Currently, the most common used ontology for the Things is Semantic Sensor Network (SSN), which was developed in 2011 [70]. Semantic Sensor Network is an effort made by several organizations to build a semantic model for the sensor network, which is to achieve the consensus between different sensor networks and the related large amount of data. The ontology is defined with the namespace "ssn: http://www.w3.org/ns/ssn/"and is currently published at [71] as a W3C Candidate Recommendation. By the ontology built in this project, all kinds of sensors, such as Weather sensor, GPS sensors, Camera sensors, Satellite Sensors can be described in a unified form. The SSN ontology contains in total eight conceptual modules for the description of sensor-related information, including deployment, system, system property, condition, feature, procedure, observation/actuation/sampling and result. 
The SSN ontology provides a good reference for the concept modelling of Things, while it only focus on the sensors, without the consideration of actuators.

IoT-lite is a lightweight ontology that was developed in 2015 as an instantiation of the SSN ontology to represent the Internet of Things (IoT) resources, entities and services.[72] The IoT-lite tries to achieve a lightweight representation form so as that the IoT platform can query the ontology without consuming excessive processing time. Moreover, IoT-lite intends to include IoT devices such as actuating devices and tag devices as a subclass of the Device concept of the SSN ontology. The IoT-lite ontology is still a working draft and is published at [73].

\subsubsection{WoT ontology}

The WoT ontology [74] is an ontology which aims to model the domain of the Web of Things according to the W3C Interest Group on the Web of Things.[75] The ontology is defined according to the examples given in the draft of the W3C current practice [76]. The ontology is not final as some aspects of the WoT current practices such as the security aspects have not been addressed yet. As a result, the ontology may change accordingly as the evolution of the W3C Current Practice draft.

\subsubsection{FOAF}

People is also an important part involved in the IoT as they are the final users of the IoT applications and devices. FOAF is an acronym of "friend of a friend", which is an ontology for describing persons, their activities and their relationships. [77] The objective of the project FOAF is to link people and information using the web. It focus on the social networks of human, friendship and association. The project was started in 2000 and the ontology has been evolving until now, which has now a stable core of classes and properties that will not be changed. The namespace URI of the FOAF is fixed as foaf: <http://xmlns.com/foaf/0.1/>. In FOAF, it defines the class foaf:Agent which is the super class of foaf:Group, foaf:Person and foaf:Organization. According to the definition, an agent can be a person, a group, a software or a physical artifact. [78] In this thesis, this concept is used to describe the person or software that sends a request to the IoT platform.

\subsection{Authentication and authorization protocols}

As security and privacy is an essential issue along with the development internet, many organizations have been cooperating to work on it. Authentication is to ensure that the agent 
which is trying to access the resource is the right agent, while authorization is to ensure that the agent has the privilege to access a/o modify the resource. Currently, the most widely used authentication protocol is OAuth standard. [79]

\section{OAuth standard}

OAuth allows the users to use their account information in a company such as Google, Facebook to access the resource in a third-party program without providing the information of username and password. The action is realized through a token-based mechanism. After a successful login, the account host company will provide the OAuth user a token, which can be given to the third-party program to access the specific resources in its server during a certain time interval. OAuth was started in 2006, along with the development of Twitter OpenID. In 2010, the OAuth 1.0 protocol was published as RFC 5849 [80]. The OAuth 2.0 was published in 2012 as RFC 6749 [81], which is supported by the Graph API from Facebook, APIs from Google and various APIs from Microsoft. OAuth 2.0 has superseded the original OAuth and becomes an industry-standard protocol for authorization. OAuth 2.0 focuses on client developer simplicity while providing specific authorization flows for web applications, desktop applications, mobile phones, and devices [82].It allows the OAuth clients to request authorization from those devices which have an internet connection but have no easy input method by performing the authorization request on a secondary device, such as a smart phone.

\section{Ongoing IETF work on Authentication and Authorization in Constrained environ- ments}

Since 2014, an IETF group Authentication and Authorization for Constrained Environments (ace) has been created, which works on seeking solutions for the authentication and authorization in constrained environments, where there are limited CPU, memory and power. REST architecture is widely used for such constrained environments. The group currently focus on applying the existing third-party authentication and authorization protocols such as the OAuth standard introduced above in the constrained environment. The group currently working on defining use cases and a draft about "An architecture for authorization in constrained environments" has been recently submitted in March, 2017. [83]

\section{JSON Web Token (JWT)}

JSON Web Token (JWT) is a compact, URL-safe means of representing claims to be transferred between two parties, which has been defined in the RDF 7519 [84]. The JWT 
token is compact and can be sent through a URL, POST parameter or the most typically in the Authorization header. The JWT token is self-contained, which means that it already contain all the required information about the user. The JWT token is typically sent in the Authorization header using the Bearer schema in the form of "Authorization: Bearer $<$ token>". The Bearer schema is part of the OAuth 2.0 Authorization Framework and has been defined in the RDF 6750 [85]. Any part that is in possession of a bearer token can used to the associated resources with out showing the possession of a cryptographic key.

RFC 6750 - The OAuth 2.0 Authorization Framework bearer token usage. 


\section{Chapter 3}

\section{Background and preliminary work}

The research presented in this thesis is conducted following the results of an IoT platform developed at the Research Centre for Smart Buildings and Energy Efficiency (CeDInt) of Universidad Politécnica de Madrid (UPM), which is part of a long-term project that has been implemented since 2011, named as BAT-System. In this chapter, the background of the research, consisting of a wireless transducer network and a software IoT platform, is introduced. Besides, the key concept "Parameter" that has been proposed and used in the IoT platform, together with the preliminary work that has been done for the interaction with the knowledge base are presented.

\subsection{Background}

Since 2008, CeDInt has been researching on the topic of home automation and started to work with traditional home automation technologies such as KNX, LonWorks, BacNet, Dali or EnOcean. These traditional technologies, though having the highest market penetration, presented several disadvantages:

- Use of proprietary protocols: others had to pay fees to develop/work products using these technologies, resulting on high cost for developers and high price for users.

- Difficult interaction between different technologies: when possible, using a gateway, with limited features.

- Not size-optimized devices, thought for pre-construction installations.

- Generally using a wired physical channel (bus, PLC).

- Software applications for specific and partial solutions. 
Considering these drawbacks, CeDInt planned the design and development of an innovative communication technology, called BATNet [86], with the following characteristics:

- Use of standard protocols.

- Wireless physical channel.

- IP identification.

- Low-cost.

- Optimized size and functionalities.

- Unified platform for third parties SW solutions.

- Based on open source HW and SW.

On the other hand, CeDInt also designed and developed a software platform oriented to enable the communication with devices of the different technologies. Its main characteristic is the use of and ontology for semantically describe the data and use for the communication technologies, data modelling, data sharing and data publishing.

\subsubsection{Wireless Transducer Network (WTN) architecture}

With the appearance of the first open source hardware and do-it-yourself platforms as Arduino [87] or Zigduino [88], it was not necessary to start from scratch on the design of the whole electronics, enabling CeDInt researcher to focus more on the communications issues. As aforementioned, the WTN development at CeDInt focused on avoiding the traditional systems limitations in terms of cost, interoperability, power consumption and collaboration. Regarding the communication network, the following protocol suite was chosen (similar architecture is currently used by other solutions), as shown in Fig. 3.1. Every WTN device communicates using standard protocols for each layer. 


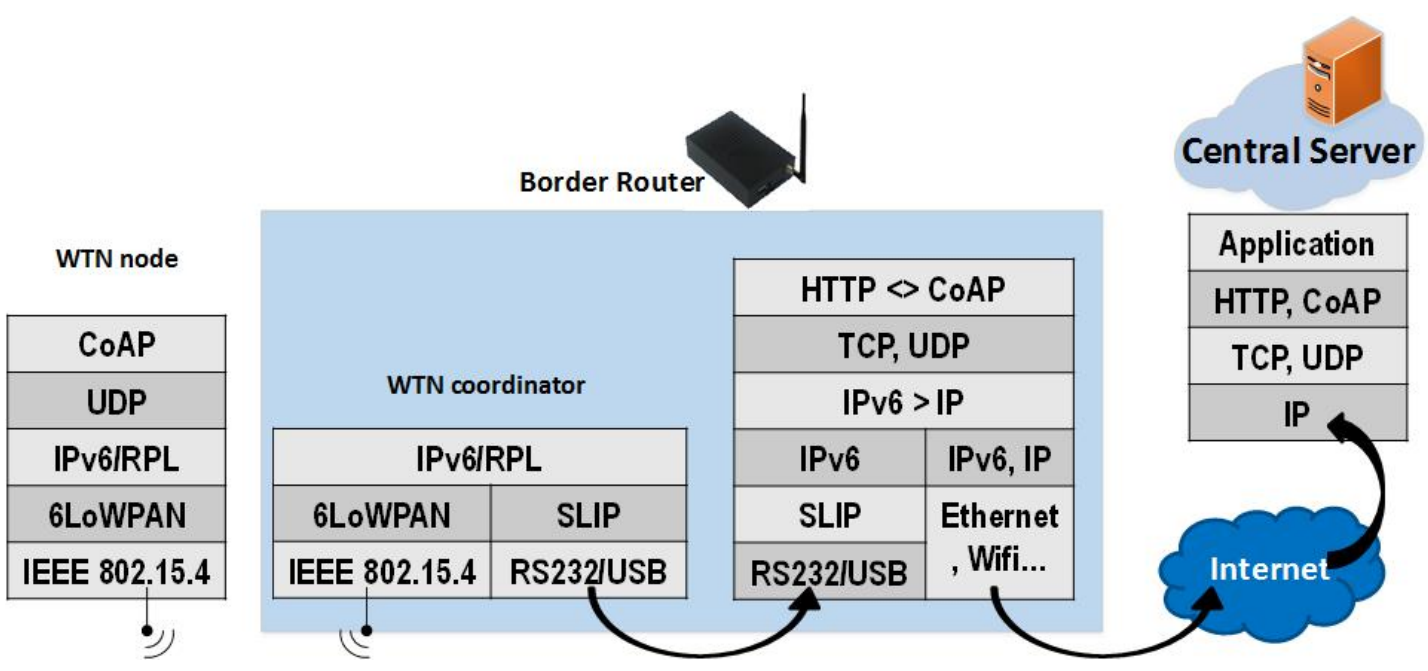

Fig. 3.1 System communication protocol stack

- IEEE 802.15.4 standard: this standard specifies the physical layer and media access control for low-rate networks. The $2.4 \mathrm{GHz}$ band has been chosen as it offers more channels and worldwide use. [89]

- 6LoWPAN-based network: 6LoWPAN (IPv6 over Low power Wireless Personal Area Networks) working group from the IETF describes the adaptation of IPv6 packets to IEEE 802.15.4 based networks. As a link layer, it adapts packets sizes and resolves addresses among other functions [90]

- IPv6/RPL: IPv6 (Internet Protocol version 6) is intended to replace IPv4, integrating the increasing number of new devices with 128-bit addresses. [91] RPL (IPv6 Routing Protocol for Low power and Loss Networks) defined by the ROLL group from the IETF, optimizes traffic patterns and minimizes routing state. [92]

- UDP: UDP (User Datagram Protocol) attributes (simple, stateless and little communication overhead) make it suitable for WSN applications.[93]

- CoAP communication interfaces: CoAP stands for Constrained Application Protocol, which is a specific web transfer protocol designed to be used with constrained nodes and networks. This protocol is simple and easy to implement. [94][95]

All the devices communicates with a Border Router which is connected into the Internet. The Border Router is a smart border router, which contains two parts:

- A WTN coordinator which interconnects the traducer network with an IPv6 network, allowing communication from external devices or clients. As it has to be always on to 
support the communications, a low power consumption and silent device is desirable. Besides, not high computing power is required since its principal task is routing low throughput data. A serial or USB port (to connect the wireless sensor network) and a RJ45 Ethernet interface (to connect the lokgcal network) are needed. A simple system on board computer with an ARM core of hundreds of MHz and $64 \mathrm{MB}$ of RAM fits all this previous requirements.

- a Java-based software program which connects to the WTN devices and communicates with a Central Server for providing users interactions with devices.

\subsubsection{Wireless Transducer Network (WTN) devices}

With respect to the hardware, the IoT solution includes a series of Wireless Transducer Network (WTN) [96] devices, including sensors and actuators, which work with the defined communication protocols. [86] The IoT network is a wireless transducer (sensor and actuator) solution based on 6LoWPAN (IPv6 over Low power Wireless Personal Area Networks) communications, enabling different types of nodes IPv6 compatible. Fig. 3.2 illustrates some of the developed WTN devices, which includes:

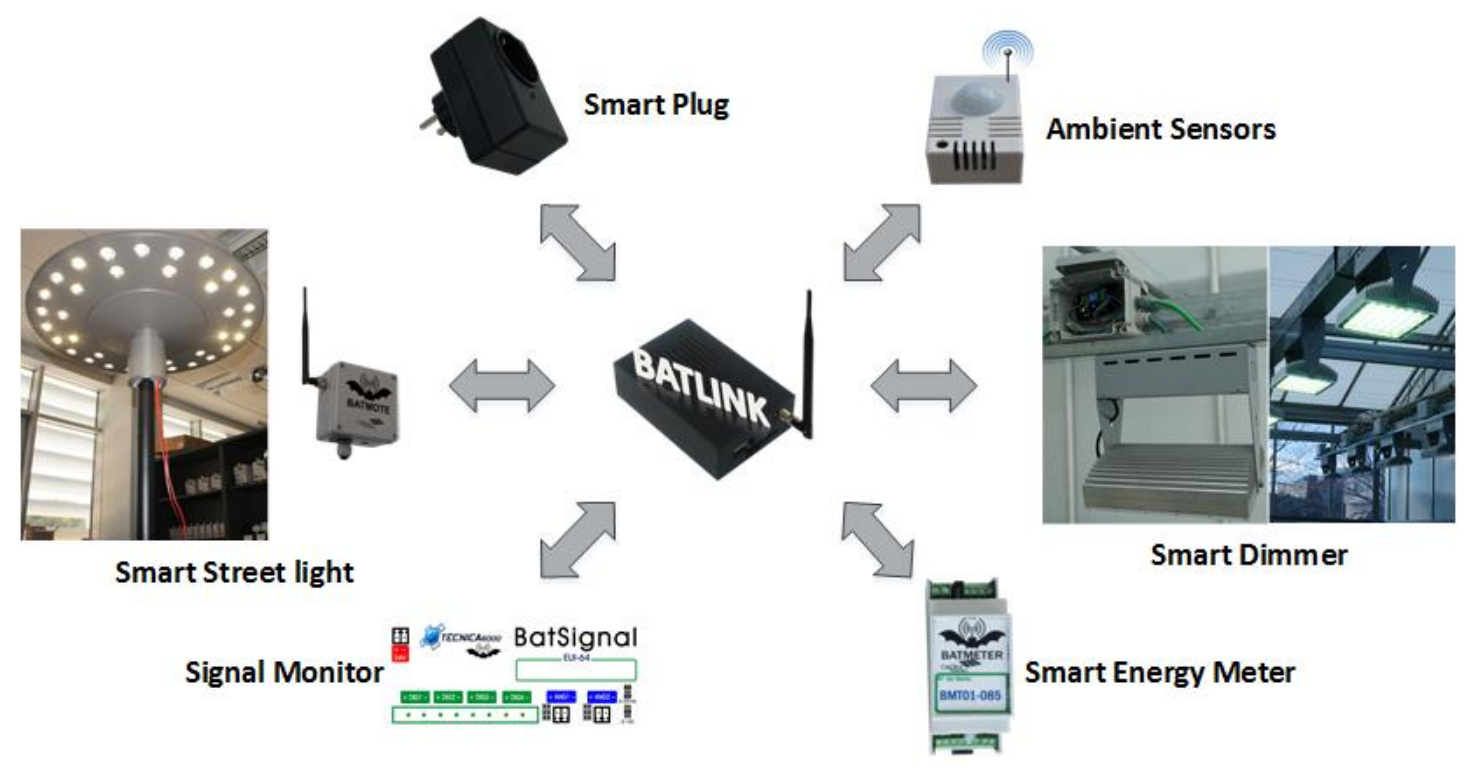

Fig. 3.2 WTN devices

- Smart Energy Meter: a device which measures the energy consumption in an electric panel.

- Smart plug: a plug which measures power consumption and acts on the loads. 
- Ambient sensors: a multi-sensor device which measures several ambient parameters such as temperature, humidity, lighting, presence and noise.

- Smart street lighting: a device designed for the outdoor public lighting. It contains a luminosity sensor, presence sensor and dimmer, which improves the lighting quality and reduces the lighting contamination and the energy consumption. [97];

- Smart Dimmer: LED lighting controller which allows to regulate the light intensity from 0 (completely switched off) to 100 (maximum LED lighting)

- Signal Monitor: a device which can detect the input analogue signal from an industrial machine and output a corresponding digital signal.

All the devices are connected through a smart Border Router, named as BATLink, which is responsible for interconnecting the WTN network and provide access to the Internet.

\subsubsection{Software platform for IoT control}

With respect to the software, a Java-based platform has been implemented to connect with the WTN devices and provide a HTTP-based interface, a Web API for the users to interact with the devices. The platform contains an ontology which adds semantic description about the devices including their functional behaviour, representation information of the measurement, location and relationship with users. Meanwhile, the platform also provides the possibility to connect with devices through other popular building automation technologies such as KNX, Modbus and EnOcean. The platform offers an abstraction layer of the technology used by the devices, i.e. Things and allows users to interact the devices through a Web API. Moreover, the platform allows the users to use the services such as energy-monitoring and ambient condition monitoring by providing different applications. [98]

The IoT platform consists of a Central Server and a set of Border Routers. The Central Server is the control centre of the IoT platform which provides a Web API for the interactions with the platform. Border Router is a smart border router with a Java-based software installed. The Border Router is to manage the devices in a local network and carry out the communication with the Central Server. A detailed introduction about the Central Server and the Border Router will be given in the following.

\section{Central Server}

Central Server is connected into the Internet, which works as the interface for all the interactions between users and the developed IoT platform and the coordinator among 
different Border Routers. Each Border Router connects with the Central Server through WebSocket. The Central Server consists of four components: Gateway Manager, Application Manager, Model Manager and Data Warehouse. Fig. 3.3 illustrates the software architecture of the Central Server.

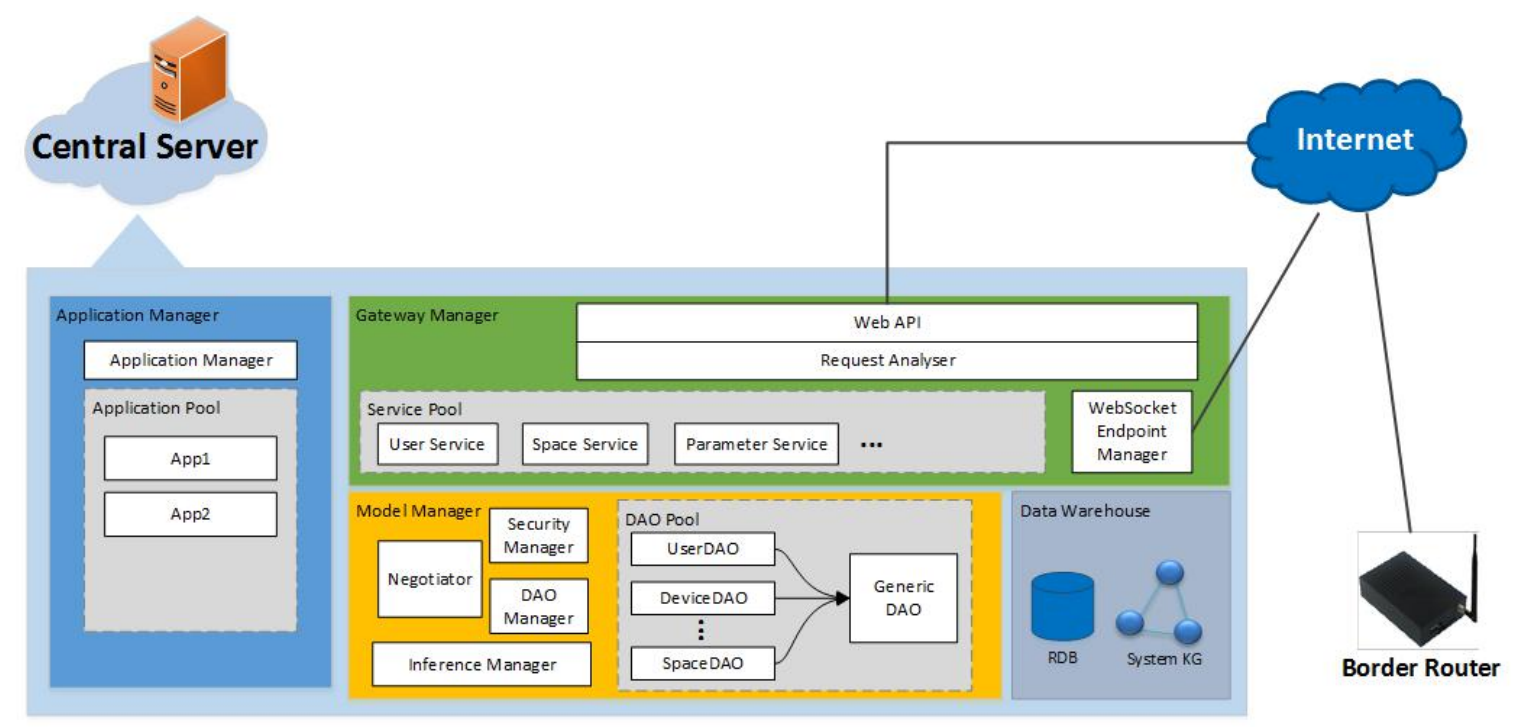

Fig. 3.3 Software architecture of Central Server

Gateway Manager A component that is in charge of the communication between external world and the platform and the communication between the Central Server and the Border Router. It contains four modules:

- Web API, a server-side web API which works as the interface to provide access to interact with the platform.

- Request Analyser, which is responsible for analysing each incoming Web API request and decide the appropriate reactions accordingly. After checking with the Security Manager of Model Manager, it either blocks/denies the request or call the corresponding service to carry out the tasks indicated in the request

- Service Pool contains a set of services. A service is responsible for performing the task indicated in a request by communicating with different DAOs.

- WebSocket Endpoint Manager maintains a set of WebSocket connections with the different Border Routers, each of which is denoted with the ID of the Border Router. If a request is to make a query or change in a local network, it will be sent to the corresponding Border Router for the execution through WebSocket. 
Application Manager A component that is in charge of the management of applications in the platform. An application is a program designed for achieving certain functions on its own initiative. In the platform, an application can take advantage of all the basic configurations of the platform such as user, device, parameters, but with extra functions such as a customized user interface or an optimized algorithm to manage the data. It contains two modules:

- Application Manager, which is responsible for installing, uninstalling and loading the applications.

- Application Pool, which contains a list of applications that are currently running.

Model Manager A component that is in charge of the interaction with the system knowledge base, i.e. the Data Warehouse. A set of Data Access Objects (DAOs) are created for performing the interaction, which will be introduced in detail in section 3.3. Both of the Gateway Manager and Application Manager need to store or query data through the Model Manager. It contains six modules:

- Security Manager, which assures the security and privacy of the platform. It works together with the Request Analyser to make sure that each request accesses the right information or devices.

- DAO Pool, which maintains a set of DAOs for interacting with the Data Warehouse

- DAO manager, which is in charge of the registration and retrieval of DAOs.

- Inference Manager, which is used to carry out inference for the System KG

- Negotiator, which is responsible for synchronizing the information among the Gateway Manager, Application Manager and Data Warehouse.

Data Warehouse A component that is in charge of the storage of data of the whole IoT platform. It contains two parts:

- System KG, which is a graph store storing the semantic data of the IoT platform as knowledge graph.

- RDB, which is a relational database for the storage of data. 


\section{Border Router}

The Border Router is connected to the internet as well as a local network. It is responsible for carrying out the communication between the Central Server and coordinating the devices in a local network. The Border Router consists of four components: Gateway Manager, Model Manager, Technology Manager and Data Warehouse. Fig. 3.4 illustrates the software architecture of the Border Router.

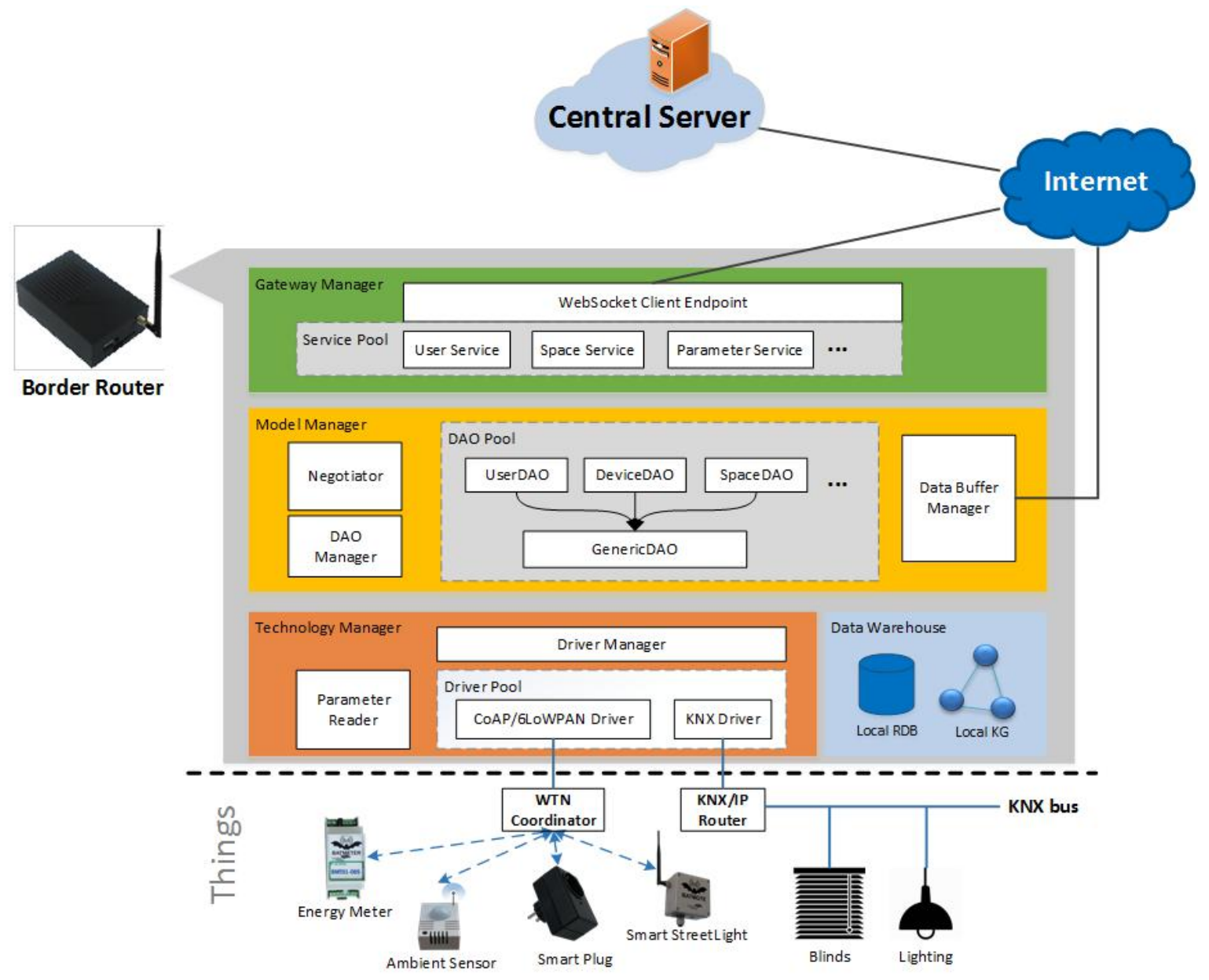

Fig. 3.4 Software architecture of Border Router

Gateway Manager A component that is in charge of the communication the Central Server and the Border Router. It receives messages from the Central Server through WebSocket and perform the tasks in the connected local network. It contains two modules:

- WebSocket Client Endpoint, which starts and maintain a WebSocket connection with the Central Server for receiving messages which indicate tasks that should be performed in the local network. 
- Service Pool contains a set of services, which performs the task received from the Central Service by communicating with different DAOs

Model Manager A component that is in charge of the interaction with the Data Warehouse. Both of the Gateway Manager and Technology Manager need to store or query data through the Model Manager. It contains four modules:

- DAO Pool, which maintains a set of DAOs for interacting with the Data Warehouse

- DAO manager, which is in charge of the registration and retrieval of DAOs.

- Data Buffer Manager, which is in charge of uploading the data generated in the local network such as measurements and device information to the Central Server by making request provided by the Web API.

- Negotiator, which is responsible for synchronizing the information among the Gateway Manager, Technology Manager and Data Warehouse.

Technology Manager A component that is in charge of the interaction with devices. It contains three modules:

- Driver Pool, which contains a set of drivers. A driver is a software program that should be installed for the communication between the devices and the Border Router.

- Driver Manager, which is responsible for loading the available drivers.

- Parameter Reader, which is responsible for performing periodical queries to devices for getting their last measurements.

Data Warehouse A component that is in charge of the storage of data for this local network. It contains two parts:

- Local KG, which is a graph store for the storage of semantic data as knowledge graph.

- Local RDB, which is a relational database for the storage of data.

\subsubsection{Applications for the developed IoT solution}

The CeDInt IoT solution has been deployed and is currently working in different projects, as illustrated in Fig. 3.5 


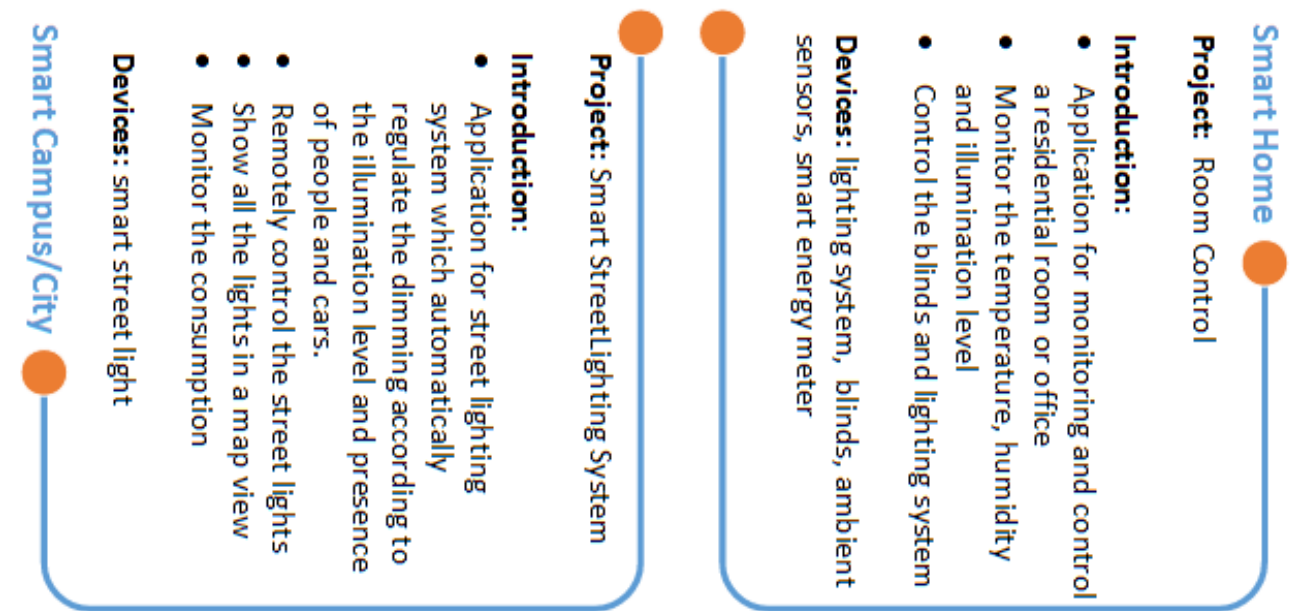

7
0
0
0
$i$
0
0
0
0
0
0
0
$\overrightarrow{0}$
$\overrightarrow{0}$
0
0
0
0
0.0
0
0
0

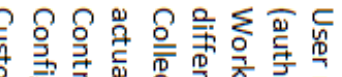

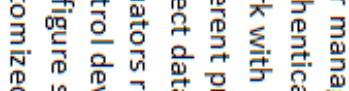

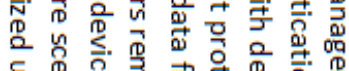

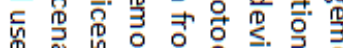

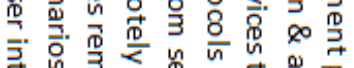

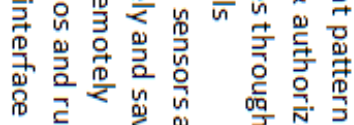

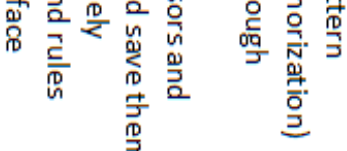
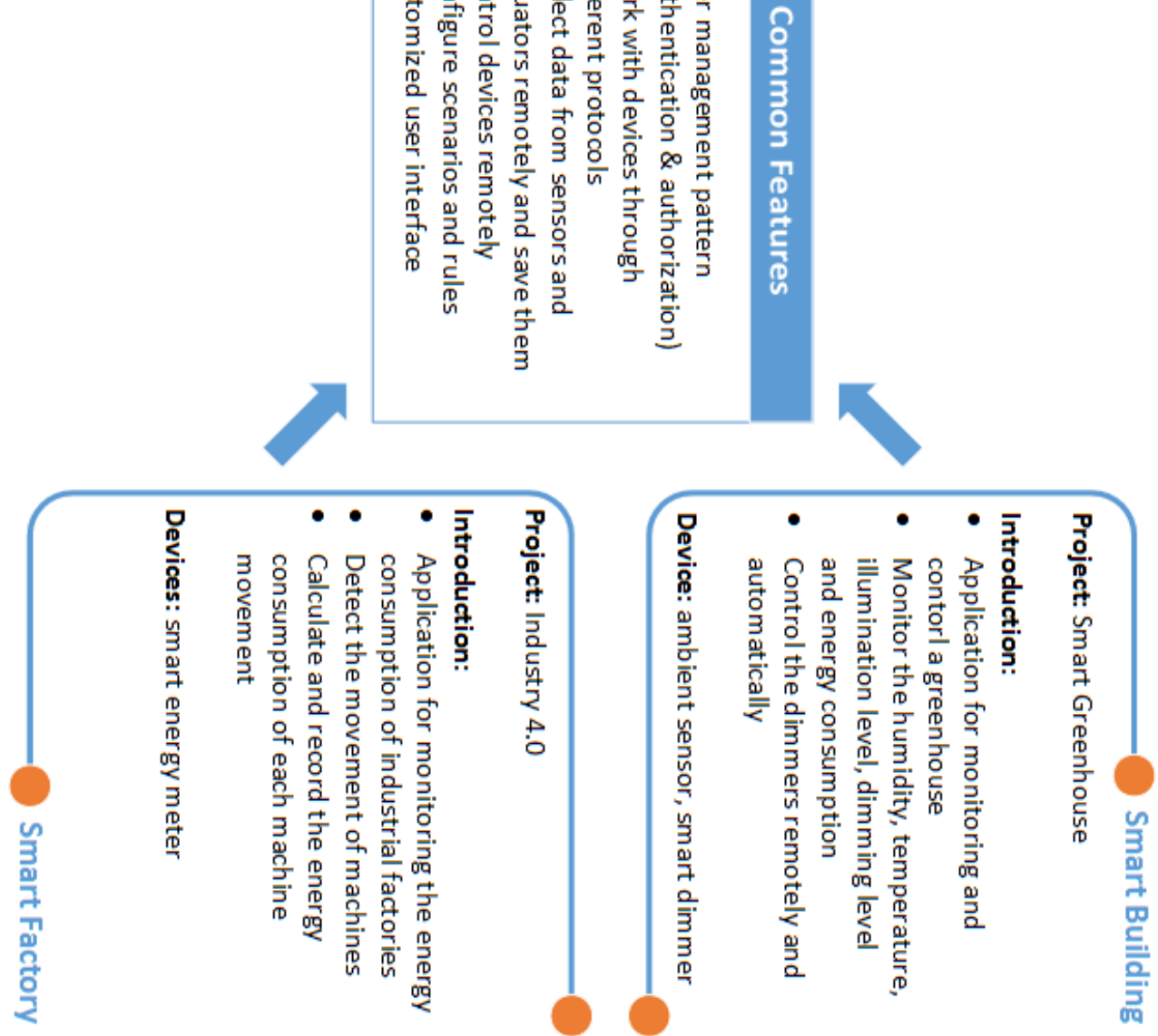
- Smart Home, application for monitoring the ambient environment and energy consumption, and control the blinds and lighting system of a residential room or office.

- Smart Greenhouse, application for monitoring and control a greenhouse by ambient sensors and remotely-controllable smart dimmers.

- Smart Campus/City, application for street lighting system which automatically regulate the dimming according to the illumination level and presence of people and cars.

- Smart Factory, application for monitoring the energy consumption of industrial factories.

All the projects share some common features, which include:

- User management pattern (authentication \& authorization), at least one user or several users should be created for the security purpose.

- Work with devices through different communication protocols

- Collect data from sensors and actuators remotely and save them in the database for the purpose of monitoring

- Remotely control devices

- Configure scenarios and rules to trigger actions of specific devices according to the time or ambient environment.

- Customized user interface for showing additional informations according to the demand of the project.

\subsection{Parameter-based Mechanism}

To facilitate the exchange and synchronization of information about the functionality, status and semantic data of Things among users, devices and applications, the developed IoT platform uses a concept "Parameter". This section will introduce about the definition of parameter in the IoT platform, the structure and its usage in both Central Server and the Border Router. 


\subsubsection{Definition of parameter}

According to the definition in Merriam-Webster dictionary, parameter is " any of a set of physical properties whose values determine the characteristics or behaviour of something". [99] In the thesis context, we define "Parameter" as "an object which wraps a physical magnitude and the information about its presentation, available actions, access type and permissions". Within the developed IoT platform, parameters are used as common objects for the communication among different platform components

Parameter is used to describe the functional behaviour of devices, i.e. Things. From the point of view of functionality, the functional behaviour of Things can be classified into two kinds, sensor or actuator. By using parameter to represent it, the sensor and actuator can be regarded equally:

- A sensor is interpreted as a non-modifiable parameter. Accordingly, "read a sensor" is performed by "query the value of a parameter".

- An actuator is interpreted as a modifiable parameter. Accordingly, "actuate an actuator " is performed by "modify the value of a parameter".

A device can be interpreted as a set of parameters if it has several functionalities, i.e. " access and control a device" is performed by "read and/or modify the values of a set of parameters". In this way, the integration of new type of devices into the IoT platform can be performed by interpreting their functional behaviour as parameters, which improves the system flexibility. Besides, this mechanism allows to compare the same function of different devices regardless the technologies used to communicate with each device.

In addition, the parameter includes the human-readable information for the understanding of system users. In this way, the parameters work as intermediate objects to wrap both of the machine-readable and human-readable information.

\subsubsection{Structure of Parameter}

The parameter is designed to deal with information exchange among users, applications and devices in the platform. Fig. 3.6 shows the class UML diagram of the parameter and its related classes.

This structure of parameter makes it possible to reach an agreement among users, devices and applications. There are in total four classes Parameter, ParameterType, Category and ParameterConfiguration, and three enumerations: ParameterPermission, ParameterDataType and ParameterAccess. A detailed explanation will be given in the following part. 


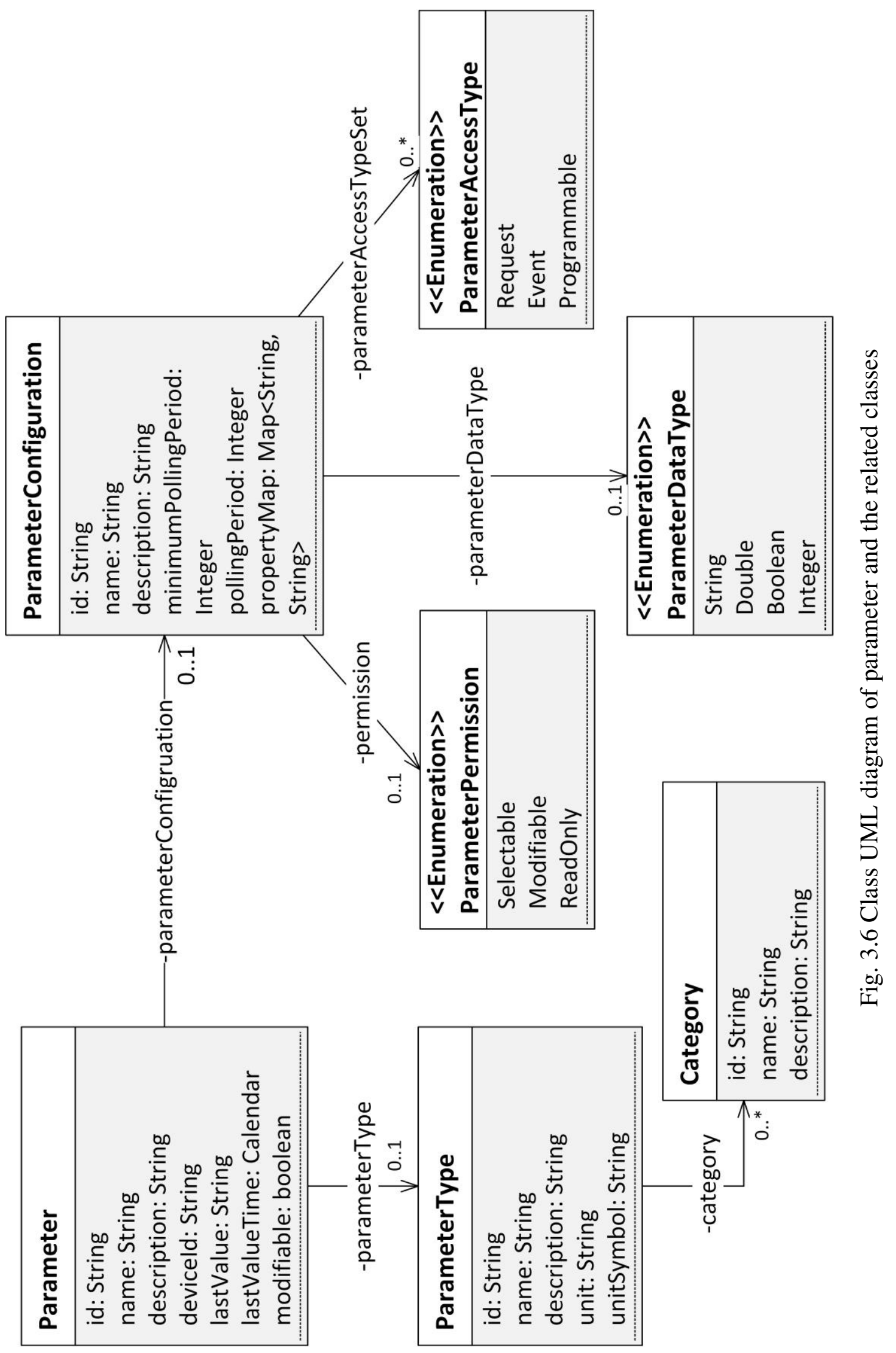




\section{Basic information}

All the classes have three basic fields: id, name and description, where:

- id refers to the identifier to identify this object, which should be unique in the knowledge base of the platform.

- name refers to a human-readable short string, which helps the user to identify the object.

- description refers to a human-readable text which gives a more detailed description about the object.

\section{Parameter}

Apart from the basic information, each parameter also has the following four attributes:

- deviceId indicates the id of the device to which this parameter belongs to.

- lastValue shows the latest value of this parameter

- lastValueTime shows the corresponding time-stamp of the lastValue

- modifiable indicates that the value of the parameter can be changed by a user. In another word, a parameter with modifiable=false represents a sensor, while the one with modifiable=true represents an actuator.

\section{ParameterType and Category}

Each parameter should have a parameter type, which indicates the meaning of the magnitude that this parameter measures and the related semantic data for its presentation. ParameterType is a way to group parameters which measure the same property of objects. The values of the parameters with the same parameter type are presented with the same unit and unitsymbol:

- unit indicates the unit of the measurement of the parameter

- unitSymbol indicates the corresponding symbol for the representation of this unit.

Each parameter type can have a list of categories. Category is a way to classify the parameter types into different groups according to their characteristics and usage, which serves the application for accomplish a certain purpose. A list of objects of Parameter Type is predefined according to the common knowledge and a list of categories has been defined on the basis of the analysis of application requirements. 


\section{Parameter Configuration}

Each parameter should has a corresponding parameter configuration, which includes all the functional arrangement about this parameter. The ParameterConfiguration class has six fields apart from the basic ones:

- minimumPollingPeriod indicates the shortest time interval that can be set between two requests to a device, which prevents the potential problem of excessive use of requests.

- pollingPeriod indicates the actual time interval between the two polling requests, which is configurable and must be longer than the minimumPollingPeriod.

- propertyMap is a map which stores a list of properties that are necessary for the driver to query or modify the value of this parameter by communicating with the device.

- permission declares that whether the parameter value can be changed. Each parameterConfiguration can only have one parameterPermission. The ParameterPermission is an enumeration with three elements:

- Selectable indicates that the value of this parameter can be modified by users and the new value should be one of a set of options.

- Modifiable indicates that the value of this parameter can be modified by users and there is not a fixed set of options to be selected.

- ReadOnly indicates that the value of the parameter can not be modified.

- parameterDataType indicates the valid format of the value of the parameter, which includes four options: String, Integer, Double and Boolean. Each parameterConfiguration can only have one parameterDataType.

- parameterAccessType indicates the form allowed by the device to query the value of a parameter. Each parameter configuration can have one or several parameterAccessType:

- Request indicates that the value of this parameter can be queried on request. If the parameter has this access type, a user or an application is allowed to communicate with the device in real time and get the value of this parameter.

- Programmable indicates that the value of this parameter should be measured at a fixed frequency.

- Event indicates that the value of this parameter is sent whenever a change happens in the device. 


\subsubsection{Parameter in Central Server}

In the Central Server, parameter is used to provide the interaction with users and applications. Fig. 3.7 illustrates the use of parameters among the components in Central Server.

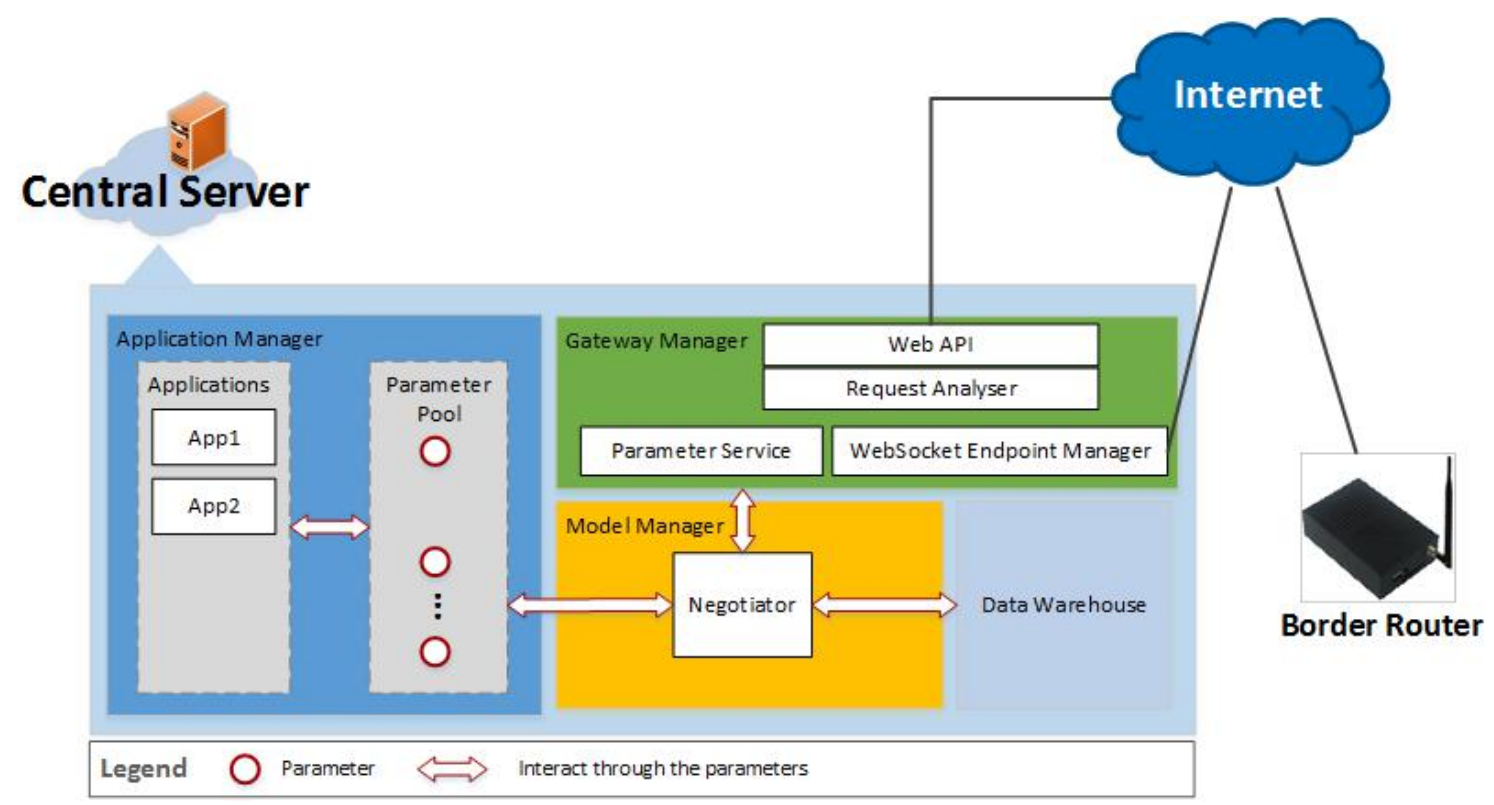

Fig. 3.7 Use of parameters among the components in Central Server

\section{Gateway Manager}

Parameter is used for user interaction in the Gateway Manager. A user can know the status of a device by ask for the value of a parameter or control a device by changing the value of a parameter. Each user can have two kinds of permissions to a parameter: reading permission, which allows the user to read the parameter value, or writing permission, which allows the user to read and change the parameter value. The parameter value can be changed only if two conditions are fulfilled:

- The parameter has to be modifiable.

- The user who made the request should have been granted writing permission over this parameter.

\section{Application Manager}

Each application can subscribe to a set of parameters. Once the subscription is performed, the application can read the value of the parameters. In the Application Manager a Parameter 
Pool is kept, which contains a list of parameter objects with their latest status including lastValue and lastValueTime. By doing this, the application can query the latest value of a parameter by asking it from the Parameter Pool, instead of querying the Data Warehouse through the Model Manager.

\section{Negotiation through Model Manager}

In the Central Server, the change of parameter value can only happen in the Gateway Manager. A user can control a device by sending a request to update the value of a parameter in the Gateway Manager through the Web API. Fig. 3.8 illustrates the workflow of the negotiation through the Negotiator of the Model Manager in this case.

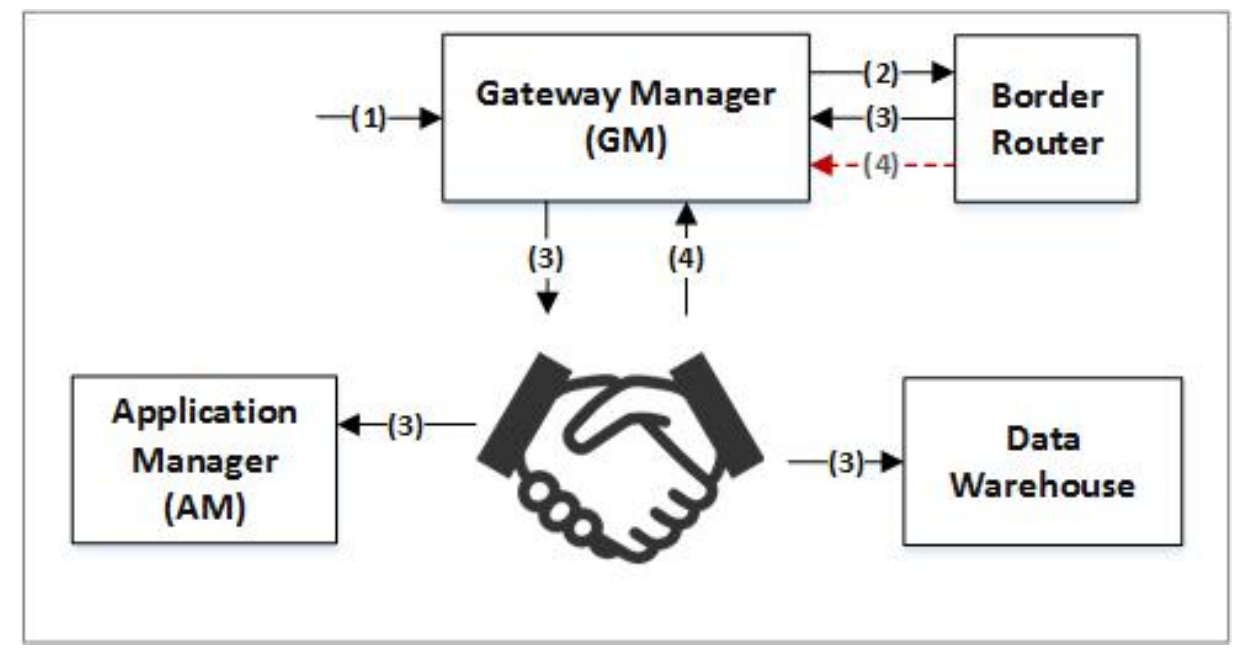

Fig. 3.8 Negotiation through Negotiator in Central Server

[1] A request to change the value of a parameter is received by the Gateway Manager.

[2] If the request is approved by the Request Analyser, a message containing the parameter id and the new value will be sent to the corresponding Border Router for changing the status of the device in its local network.

[3] If a success message is returned by the Border Router, the Gateway Manager will send a message containing the parameter id, the new value and the time-stamp to the Negotiator, which will then inform the Application to update the value of the corresponding parameter in its parameter pool and store the new value in the Data Warehouse.

[4] If an error message is returned by the Border Router, the Gateway Manager will return the message to the user. 


\subsubsection{Parameter in Border Router}

In the Border Router, parameter is used to provide the interaction with devices and the Central Server. Fig. 3.9 illustrates the use of parameters among the components in Border Router.

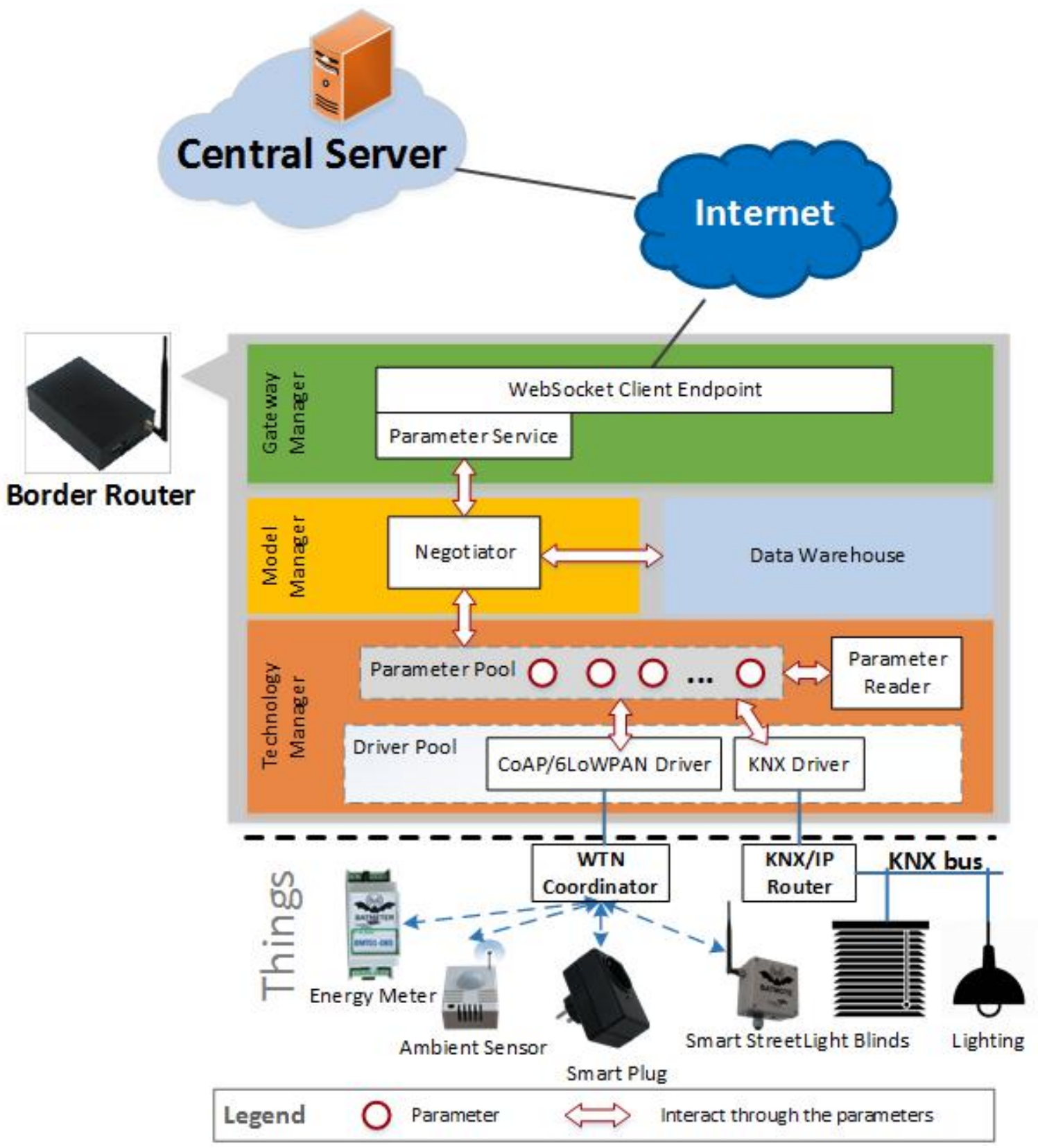

Fig. 3.9 Use of parameter among the components in Border Router 


\section{Parameter with Technology Manager}

In the platform, different drivers are implemented for connecting to devices through different communication protocols. A communication protocol defines a list of rules and the data format for exchanging messages among devices. The device can be a sensor, an actuator or a computer. Each device sends or receives messages following one or several communication protocols. The function of a driver is to map a device to one or a set of parameters according to its functional behaviour and its configuration. The driver query the device and interpret/parse the received data as the values of corresponding parameters.

In the Technology Manager a Parameter Pool is kept, which contains a list of parameter objects with the information about their configuration data and the latest status including lastValue and lastValueTime. This Parameter Pool only contains the parameters that have Programmable as their ParameterAccessType. An independent process Parameter Reader reads the lastValueTime of each parameters in this pool and compare it with the current time. If the difference are long than the value of its pollingPeriod, a new query will be sent to the corresponding device to get the latest value of this parameter.

\section{Parameter with Gateway Manager}

If a user sends a request to the Central Server to update the value of a parameter, it will then send a message containing the parameter id and the new value to the Gateway Manager of the corresponding Border Router. If such a message is received, the Gateway Manager will then communicate with the Technology Manager through the Negotiator to carry out the task.

\section{Negotiation through Model Manager}

In the Border Router, the change of the parameter value can happen in the Gateway Manager or Technology Manager, which should be informed to the Negotiator of the Model Manager so as to synchronize the information with other components. Fig. 3.10 illustrates the process of negotiation through Model Manager. 


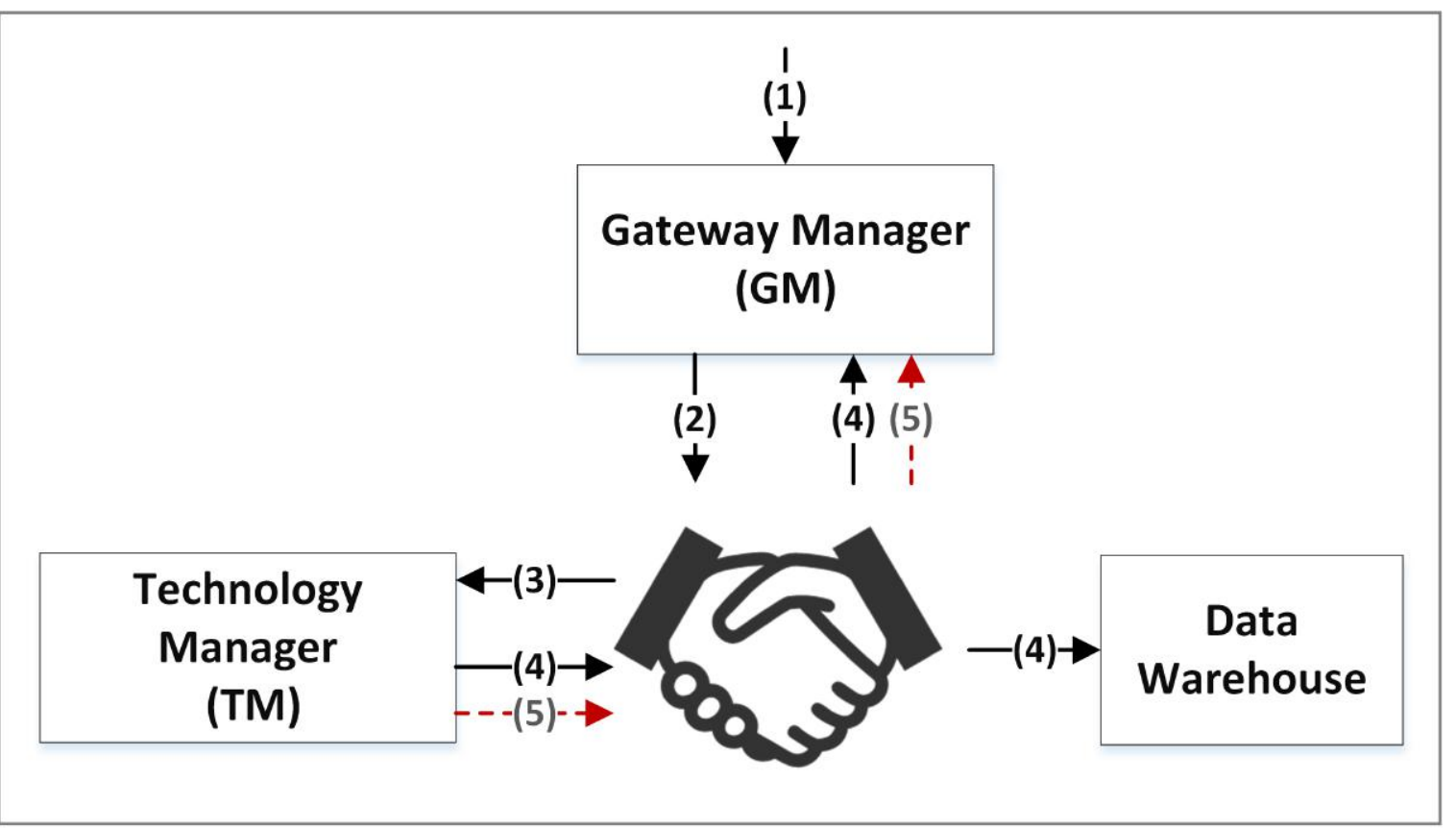

(a)

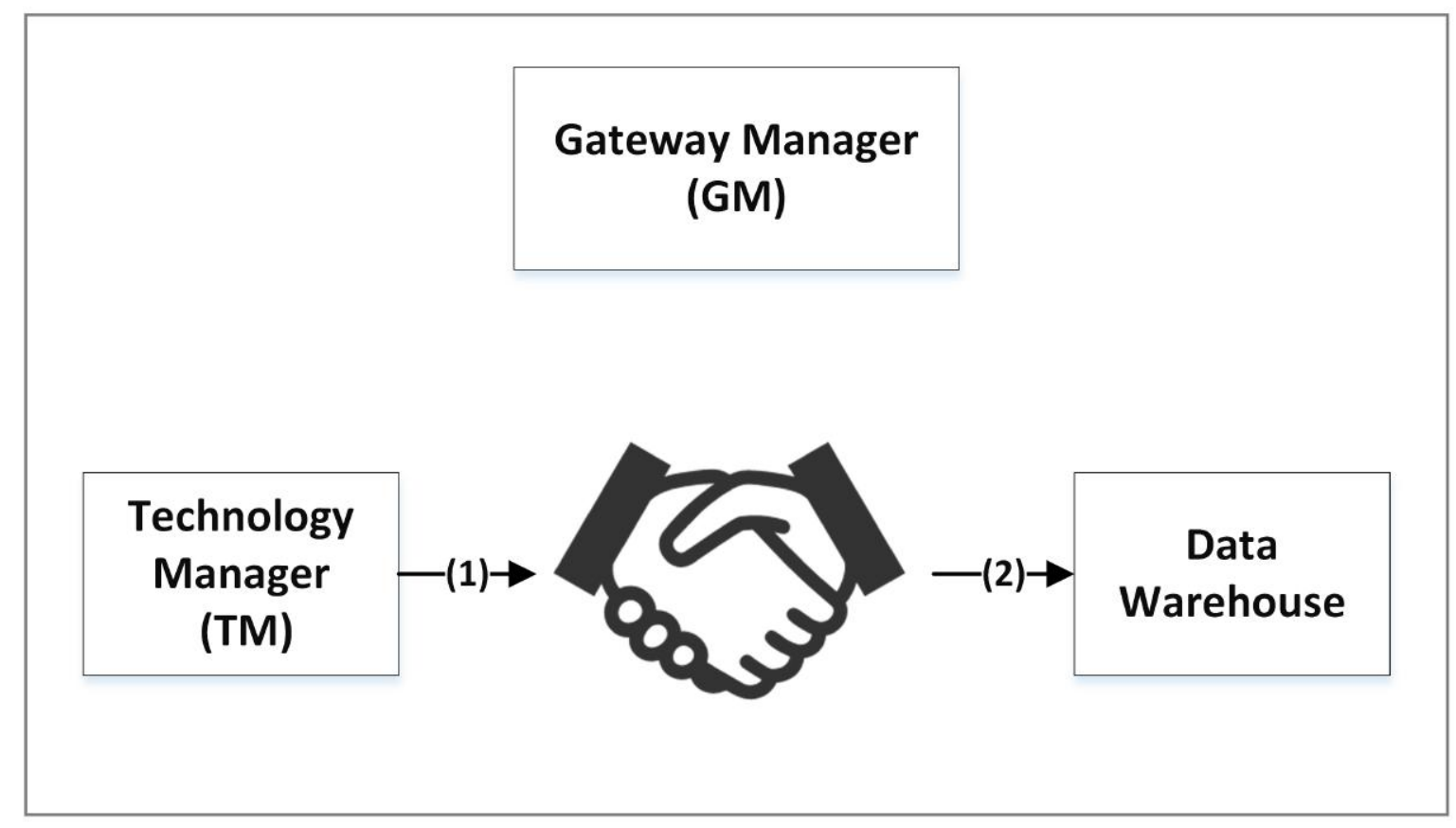

(b)

Fig. 3.10 Negotiation through Negotiator in Border Router 
Change value of a parameter through Gateway Manager If a user wants to change the value of a parameter, the Central Server will send the request to the corresponding Border Router, as illustrated in Fig. 3.10(a):

[1] One message, containing the id of the target parameter and the new value to be set, arrives at the Gateway Manager.

[2] The Gateway Manager send the id and new value to the Negotiator.

[3] The Negotiator sends the id and new value to the Technology Manager, and the Technology Manager communicates with the device corresponding to this parameter through the driver to change its status in the physical world.

[4] If the value is set successfully, the Technology Manager will send a success message to the Negotiator, containing the new value and time-stamp. The Negotiator then sends the success message to the Gateway Manager to send back to the Central Server and save the new value in the Data Warehouse.

[5] If the value fails to be set, the Technology Manager will send an error message containing the cause to the Negotiator, which then sends the error message to the Gateway Manager to send back to the Central Server.

Change of parameter value in Technology Manager In the Technology Manager, The value of parameter is updated due to the periodical query to the devices by the Parameter Reader. As illustrated in Fig. 3.10(b):

[1] When the value of a parameter changes, TM sends a message containing the new value, the parameter id and the time-stamp to the Negotiator.

[2] The Negotiator stores the new value of the parameter in the Data Warehouse.

In conclusion, with the introduction of the concept Parameter, the consistency of information about Things is assured among all the components, which provides an important foundation for the interactions and operations in the platform.

\subsection{Interaction with Data Warehouse}

Both of Central Server and Border Router has a Data Warehouse, where the data generated in the platform is stored. All the interactions with the Data Warehouse are carried out by the Model Manager. The developed platform is a Java-based software platform. An annotation 
based mechanism has been designed to manipulate the data in the Data Warehouse through the Model Manager.

\subsubsection{Data Warehouse}

The Data Warehouse works as the knowledge base of the platform. The Data Warehouse consists of two parts, a MySQL relational database and a Graph Store, provided by Jena TDB. This decision has been made on the basis of the analysis of characteristics of these two different kinds of storage method:

- Speed of Processing: In [100], it is illustrated that a relational database such as MySQL has a highly superior performance in processing speed than those ontology triple stores such as JenaTDB and Virtuoso. both in load time of triples and the query processing execution.

- Data sources integration: One of the key contribution of Ontology is to create a common vocabulary in a specific domain for easy knowledge sharing and reusing. As a result, it is able to integrate the information from different data sources easily. While relational database is not designed for realizing this function.

- Data publishing: A triple store can provide a SPARQL end point for easily publishing and sharing data, while relational database is a relatively closed environment and do not dedicate to publish the data contained.

In the case of the platform, it is designed for integrating different kinds of data, including the data of user, spaces, devices and measurements. For different data, the requirement for data storage is different. The decision is made on the consideration of three aspects:

- If the data can be exposed. Those data that concerns about security and privacy including the authentication data, personal contact data should not be published, which should be stored in the relational database.

- If the data can make contribution to if it is shared with others. With respect to users, the person-description information such as age and gender is more appropriate to be stored in the ontology for data publishing and further data mining such as user behaviour analysis.

- If the data need to be published. With respect to the devices, the configuration data that are used for the local communication have no interest to be published, while the measurements of the devices and their related semantic information can be published and shared to benefit other entities who needs these data. 
As a result, it was decided to apply both of the storage manners in the platform Data Warehouse for storing different data according to their usage and properties. Nowadays, some work have been done to develop technologies for mapping the data or convert the format between relational databases and semantic ontology triple stores[101]. But there is rarely methodology to realize the mapping or unifying of these two kinds of storage in the software level. On the consideration of this demand for the platform, a preliminary work has been done which designs a mechanism for accessing, persisting and managing data between Java objects and a knowledge base which contains a relational data base and a graph store simultaneously. The persistence of Java objects refers to the process to store them in a permanent storage.

\subsubsection{Data Access Objects (DAOs)}

Data Access Object is a software design pattern introduced by Sun Microsystems for providing data operations without exposing details of the database [102]. Data Access Object is an interface used to provide access to the persistent storage layer. This makes it possible that the changes of logic at the storage side will not affect the operation of other modules. These changes could be database type (relational databases, ontology, flat files), database driver (MySQL, SQLite, Jena TDB, Virtuoso) or database location. In general, by using DAOs, the process of storage is eased and separated from the main business logic of the system.

\section{Use of Generic DAO}

In the platform, Each DAO is responsible for handling one specific class. For instance, User DAO is responsible for User class, and ParameterDAO is responsible for the Parameter class. Following the rule of "not repeating code", a Generic DAO is constructed for carrying out the basic data CRUD (Create, Read, Update, Delete) functions and storing the key information of persistent storage. Generic DAO works with the generic type T [103] and provides five basic functions by using Java Reflection programming [104]:

- insert, create a new object in the Data Warehouse

- update, update the data of the object with the same id in the Data Warehouse

- delete, delete the object with the given id

- findById, get the object of this class with the give id

- findAll, return all the objects of this class 
Each DAO inherits the five basic functions from Generic DAO and contains its own methods according to the different needs for handling the data of the corresponding class. In the case of UserDAO, a method authenticateByPassword() is provided for validating the authentication information of users. Fig. 3.11 shows the construction of Generic DAO and an example DAO, User DAO.

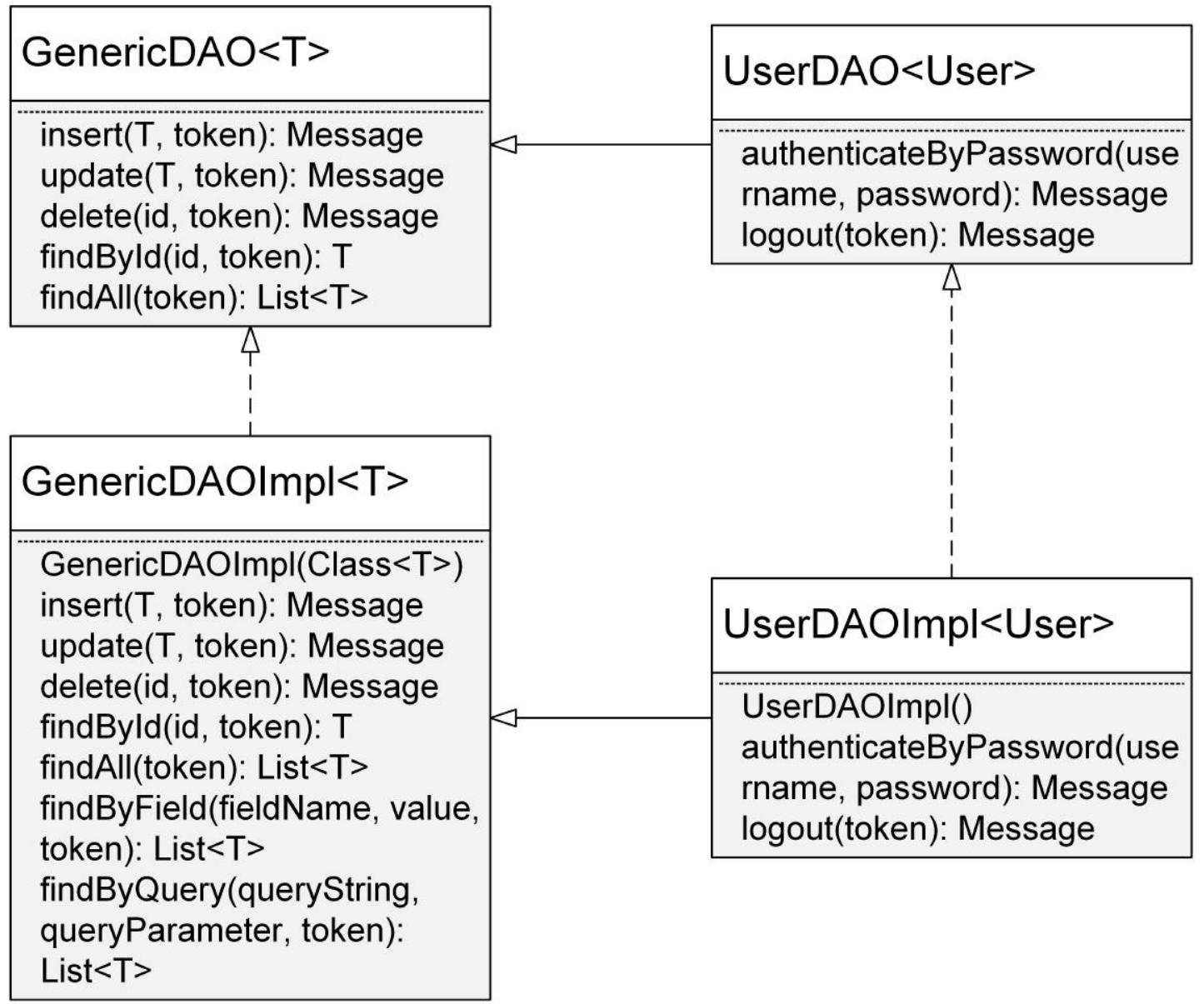

Fig. 3.11 Generic DAO and DAO structure

Besides the default five methods, GenericDAOImpl also provides a list of methods for the general use purpose, such as findByField() and findBySPARQLQuery():

- The method findByField() allows to get a list of objects whose specified field is equal to a given value.

- The method findBySPARQLQuery allow to get a list of objects by executing a specific SPARQL query. 
Each DAO is able to connect to one relational database and one ontology triple store at the same time. All the DAOs are kept in a DAO pool and each component retrieves those DAOs that interest it from there.

\section{DAO creation, registration and retrieval}

For creating and retrieving DAOs properly and efficiently, a DAO Manager is employed. This DAO Manager maintains a list of mappings between the Java classes to be persisted and their corresponding DAOs. For instance, for persisting the instances of User class, User DAO is needed. Every time a DAO is created, it needs to be registered in the DAO Manager by adding a mapping between the target class and the DAO. In case that a class has subclasses, the mapping between each subclass and the DAO should also be added. Sometimes, an instance may contain variables which are instances of other classes. In this case, during the reading and writing process, the DAO Manager will first search its mapping list. If a corresponding DAO is found for this object, this DAO is applied. If no proper DAO is found, a generic method is provided for persisting the field. For instance, a user contains a list of roles. As the Role DAO is registered, it is used for handling the role instances inside the user instance. As each DAO contains some basic information such as id field, table name and individual URL prefix. which needs to be obtained by looping all fields of the class. This mechanism helps to reduce the iteration process so as to work more efficiently.

\subsubsection{Creation of new annotations}

In Java, annotations are a form of metadata which provide data about a Java program that is not part of the program itself. Annotations can be used to provide the information for compile-time and deployment-time processing and runtime processing. [105] It is possible to create meta-annotations out of the existing ones in Java. [106] On consideration about the demands expalined above, a mechanism has been designed to instruct the Model Manager about where and how to persist a Java object by creating a set of new annotations for the use in this developed IoT platform.

The Java Classes to be persisted should follow the pattern of Plain Old Java Object (POJO), i.e. only have variables and the getters and setters for each variable. As these objects should be a reflection for the data persisted in the Data Warehouse, this convention is not going to result in sacrificing of expressiveness.

By adding annotations in one Java class, the class can be integrated with the platform and benefit the data persistence process of Model Manager without changing its class structure. 
The instances of the Java class will be persisted to relational database or ontology triple store according to their annotations.

The new defined annotation set (Fig. 3.12) consists of three parts: general annotations, annotations for relational database and annotations for ontology. By the creation of this annotation set, the issues including data storage instruction, ontology concepts registration and most importantly, data consistency guarantee in Data Warehouse has been solved .

\section{Data Storage instruction and configuration}

Having a class to persist, the first problem to solve is which storage(s) to choose. For telling this, we have defined two class-level annotations, @OWLClass and @ RDBClass.

- If a Java class needs to be persisted partly or fully in the ontology triple store, the annotation@OWLClass should be provided with the corresponding concept name, such as@OWLClass("bat:User"). The annotations @OWLDatatypeProperty and @OWLObjectProperty inside of a class will be ignored if the class does not have @OWLClass.

- If a Java class needs to be persisted partly or fully in the relational database, the annotation@RDBClass should be provided with the corresponding table name. A class with @RDBClass("user") will be persisted to the table named "user" in database. The annotation@RDBAttribute inside of a class will be ignored if the class does not have @RDBClass.

\section{Concept Registration in the platform}

For each class to persist to ontology, by providing the information in @OWLClass and @ Namespaces, it is able to register a series information in the ontology structure of the platform:

- @ Namespaces provides a list of mapping between the prefixes and the corresponding URL such as "bat:www.cedint.upm.es/bat.owl $\sharp "$. If the defined prefix is already registered with another URL, an exception is thrown.

- @ OWLClass("conceptName") provides the corresponding concept URL of the class in the ontology. 


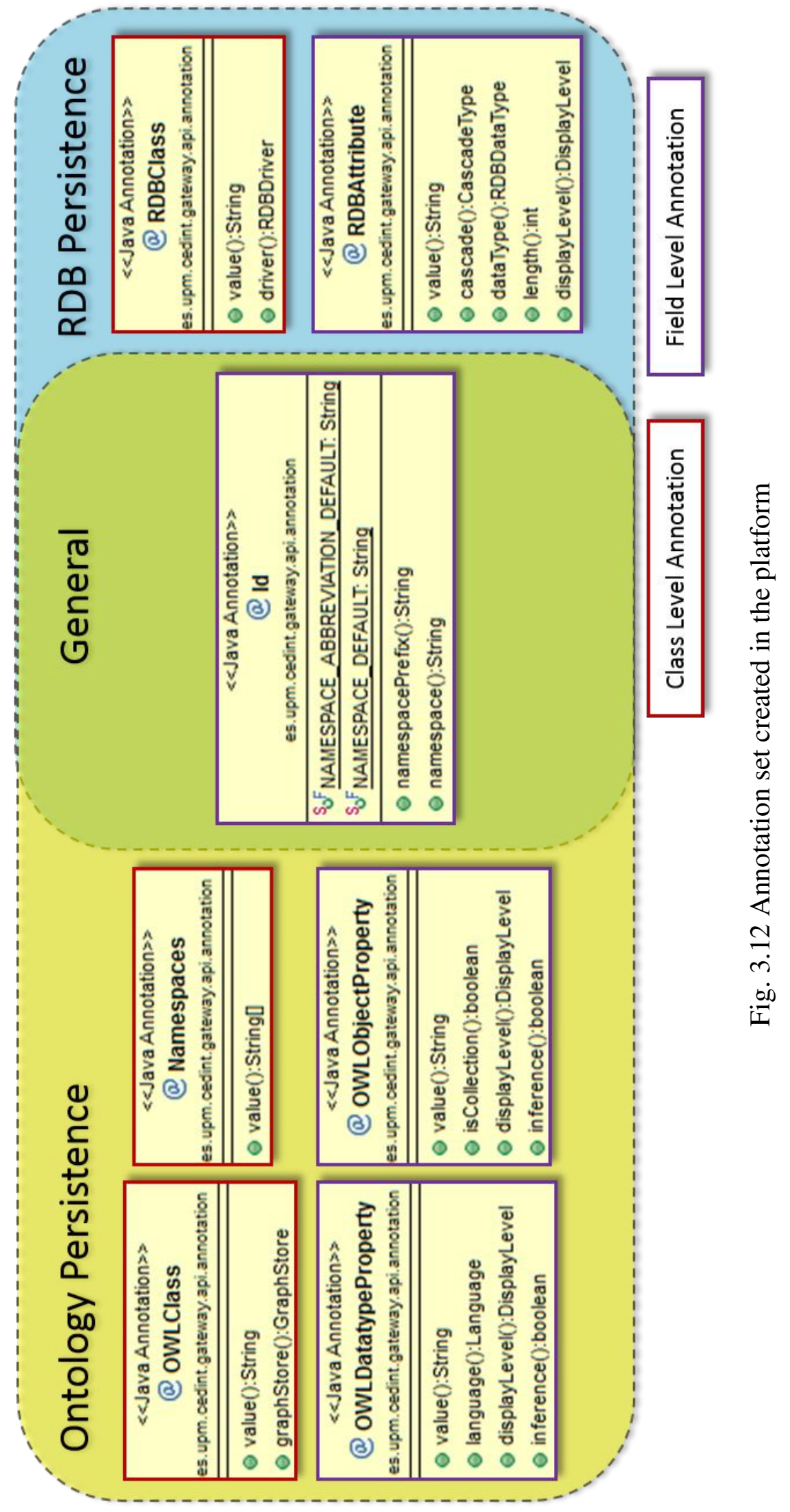




\section{Data consistency between ontology and relational database}

In the case that one class is stored in both ontology and database, an unique identifier is necessary for identifying the same object in both of these two storage. Fig. 3.13 illustrates how the same Java object is persisted into both storages.

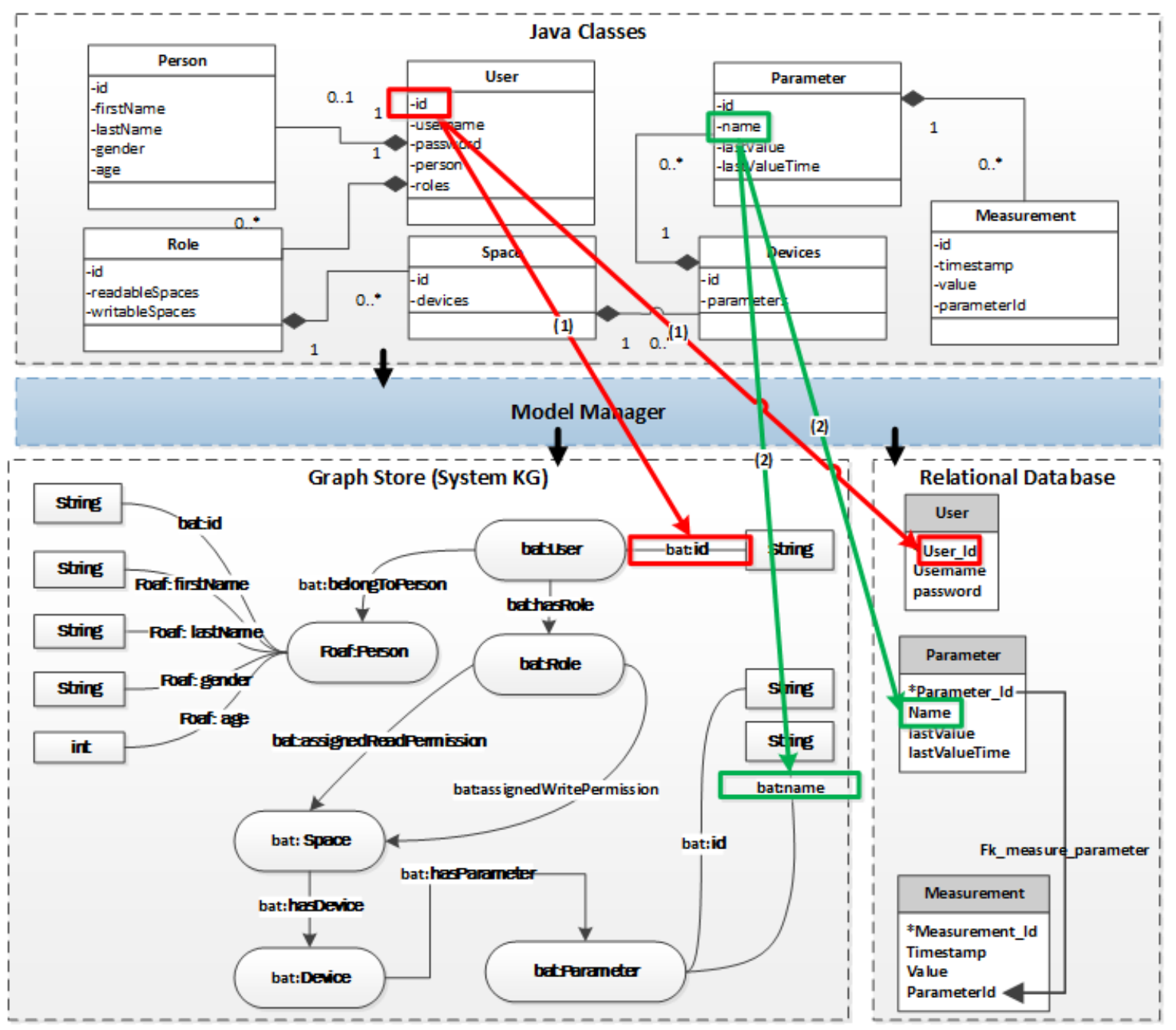

Fig. 3.13 Ontology storage and relational database schema

- The annotation @ Id is defined for achieving this objective. It is obligatory to put @ Id for one and only one Primitive/Primitive Wrapper field of the class. The annotated field will be used as the primary key in the database and as the reference to generate individual URL in the ontology. During the persistence process, data consistency is guaranteed as the data come from the same object, as shown by theow (1).

- During the retrieval process, Model Manager retrieves the data of each fields according to the same id passed and unifies them in the same object. If the data of the same field 
is different in the two storages, an exception will be thrown. As shown by the arrow (2), if the value retrieved from the System for property bat:name and value retrieved from RDB for column Name are different, an exception will be thrown.

\section{Storage of attributes of concepts and relations among them}

In a Java class, one field could be of type Primitive/Primitive Wrapper or an object/collection of objects:

- If the field is of type Primitive/Primitive Wrapper, it will be stored in the ontology as an attribute of the concept if the annotation @ OWLDatatypeProperty provided, while if $@$ RDBAttribute is provided, the field will be stored as a column in the corresponding table.

- If the field is an object or a collection of objects, if a @OWLObjectProperty() is provided, this relationship will be stored as an object property between the annotated field and the current class. While, in relational database, the cases could be more complicated. Considering the different cases of fields inside of a Java class, an option cascade() is provided for @ RDBAttribute to indicate the manner used to store the data:

- CascadeType.TOSTRING, indicating that the field is converted as a String to be stored in the corresponding column.

- CascadeType.MERGE, indicating that all the fields inside of this annotated field will be retrieved and stored in the same table of the class.

- CascadeType.RECURSIVE, indicating that the annotated field will be stored in a separate table with a foreign key consistent with the primary key of the current class.

\section{Data Retrieve confidentiality and efficiency}

Considering the efficiency and security of data retrieval, an option displayLevelis provided in both of the annotations. It indicates the level to which the field value is exposed:

- DisplayLevel.CONFIDENTIAL, for the confidential information such as a password or a secret key.

- DisplayLevel.PUBLIC, for the fields that should be shown or retrieved in all kinds of methods. 
- DisplayLevel.SPECIFIC is the default option, which is used for those field that should not be exposed when a list of objects are retrieved at the same time.

In Model Manager, those methods for retrieving data can define the displayLevel. The information will be exposed only if its displayLevel is higher than the level specified in the retrieve method, the sequence is PUBLIC > SPECIFIC >CONFIDENTIAL.

\subsubsection{Data persistence and retrieval}

Model Manager is used to ensure the data sent by the different the components are stored correctly in Data Warehouse and also to ensure that the data stored there are queried in an effective and secure way by each component. For easing the process of data persistence,the software pattern Data Access Object (DAO) is adopted.

\subsubsection{Control of Data Consistency by DAOs}

As the annotation contains all the information about exactly where and how to persist the data, the initial database and ontology can be empty unless some backup file is desired to be loaded first. In the following part, the collaboration between DAO and the annotations will be explained. The Data Consistency is guaranteed by the DAOs from three aspects.

Data Consistency during initial step. Database/Ontology construction This automatic construction mechanism prevents the potential inconsistency among a manually created database, ontology structure and the persisted Java class. When creating a DAO, the target class $\mathrm{T}$ should be assigned to the DAO. Once the registration has taken place, the DAO checks the annotations of T. If @OWLClass is found, a new concept corresponding to this class will be created in the ontology, while if @ RDBClass is found, a table will be created in the relational database. Hereafter, the DAO scans each field until it finds the one with@ @ Id. This field will be to generate the individual URL in ontology and/or used as primary key in the database. Finally, the DAO scans all the fields and creates the properties in ontology and/or the columns in the database accordingly.

Data Consistency during Data Storage The data storage is accomplished by the methods insert() and update(). When a new instance needs to be persisted, the DAO reads first the identifier field and create a new individual in ontology and/or a new row in database accordingly. Afterwards, the DAO reads all the fields, adding new properties and/or updating the row accordingly following the annotations. If it succeeds, a success message containing 
the object id will be returned. As the information for storing in both place come from the same instance, the data consistency is guaranteed. If it fails, an error message containing the cause will be returned followed by a roll-back in the interaction with both of the storage. In this case, the data consistency is assured.

Data Consistency during Data Retrieval The data retrieval is accomplished by the methods findById() and findAll(). When the method findById() is called, the DAO reads all the fields of the target class and constructs Ontology query (SPARQL) and/or database query (SQL) according to the annotations. After making the queries, the DAO creates an instance of target class and assigns the retrieved values to each corresponding field. If it succeeds, the instance is returned. If the given object id is not valid, a null will be returned. If the data retrieved from the two storage is not consistent, an exception will be thrown during the data retrieval process, which ensures the Data Consistency.

\subsubsection{Example Class}

Fig. 3.14 illustrate how to use the annotations created with an example.

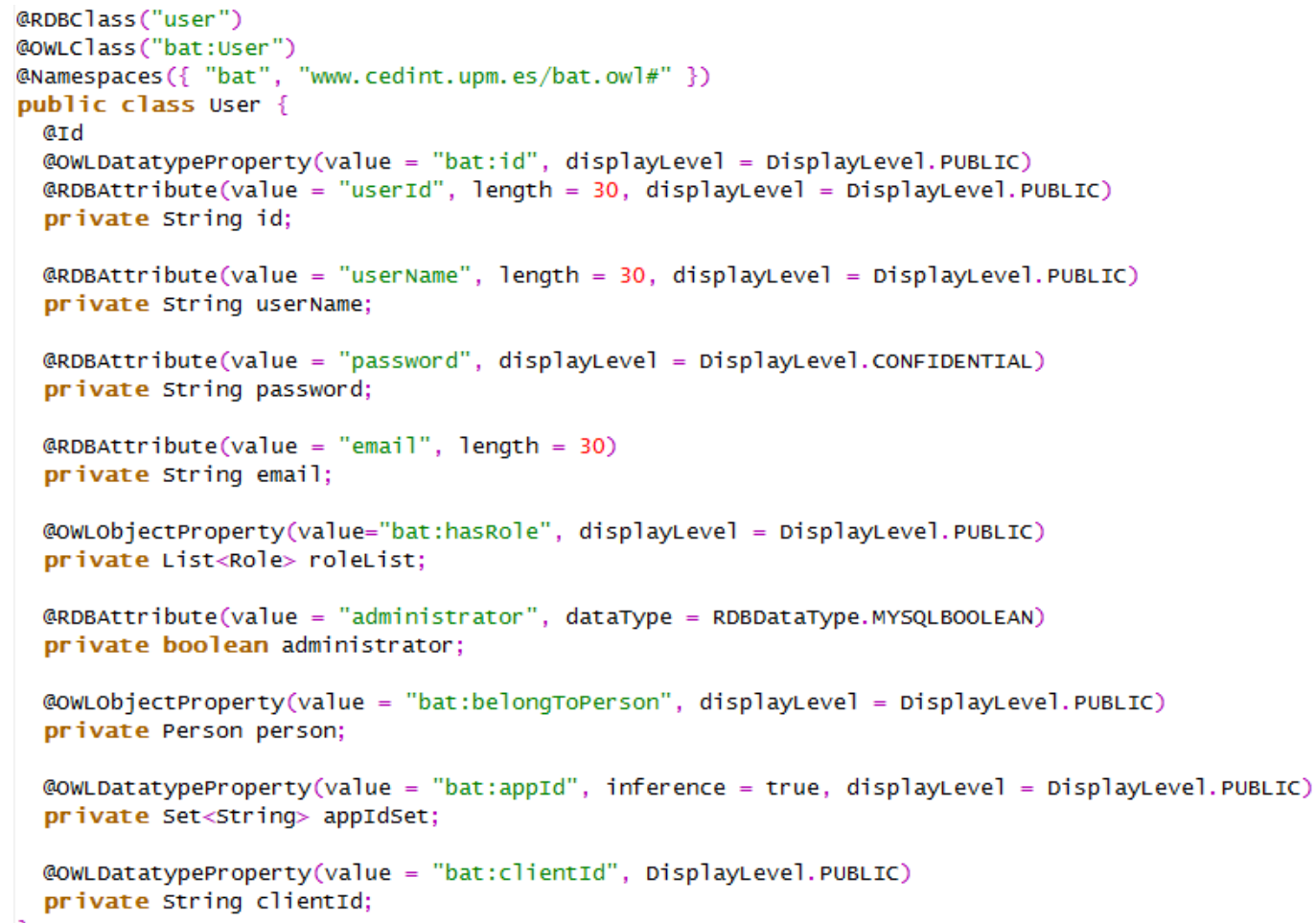

Fig. 3.14 Java class User annotated with the created annotations 
The Java class User is persisted both in the relational database (table "user") and the graph store (instance of "bat:User"). The field id, username, password, email, administrator will be persisted in the relational database, while the field roleList, person, appIdSet and clientId will be persisted in the graph store.

The designed mechanism in this section has been applied in the developed IoT platform as an preliminary work of the focus of this thesis, which accomplishes the interaction between the other components with the Data Warehouse. 


\section{Chapter 4}

\section{System KG and Inference}

In the developed platform, a series of Semantic Web technologies including ontology engineering, inference and SPARQL are applied for the implementation of an authorization mechanism. This chapter will give a detailed introduction of System KG and the process of inference.

\subsection{Overview of the System KG}

System KG is the knowledge base of Central Server, which is responsible for the storage of all knowledge graphs. System KG contains the information about all the entities involved in the platform, such as users, devices and spaces. System KG consists of three parts: Original KG, Deductions KG and a set of PKGs.

- Original KG contains the statements that describe all the asserted fact in the platform.

- Deductions KG contains the obtained results of the inference, which is the implicit information that has been inferred by Rule Engine following a set of rules according to the fact provided by the Original KG.

- The union of Original KG and Deductions KG forms the Inference KG, which is used as the knowledge base for generating PKGs. Inference KG is stored in the form of dataset which consists of an amount of named graphs. [54]

- PKG stands for "Private Knowledge Graph", which is a new concept that has been proposed in this thesis for achieving fine-grained access control. PKG is a knowledge graph generated for the use of a specific agent, either a user or a Border Router. It is a subset retrieved from the System KG following some policies and contains exclusively all the information that this agent is authorized to access. 
Fig. 4.1 shows the workflow of the PKG generation for an agent.

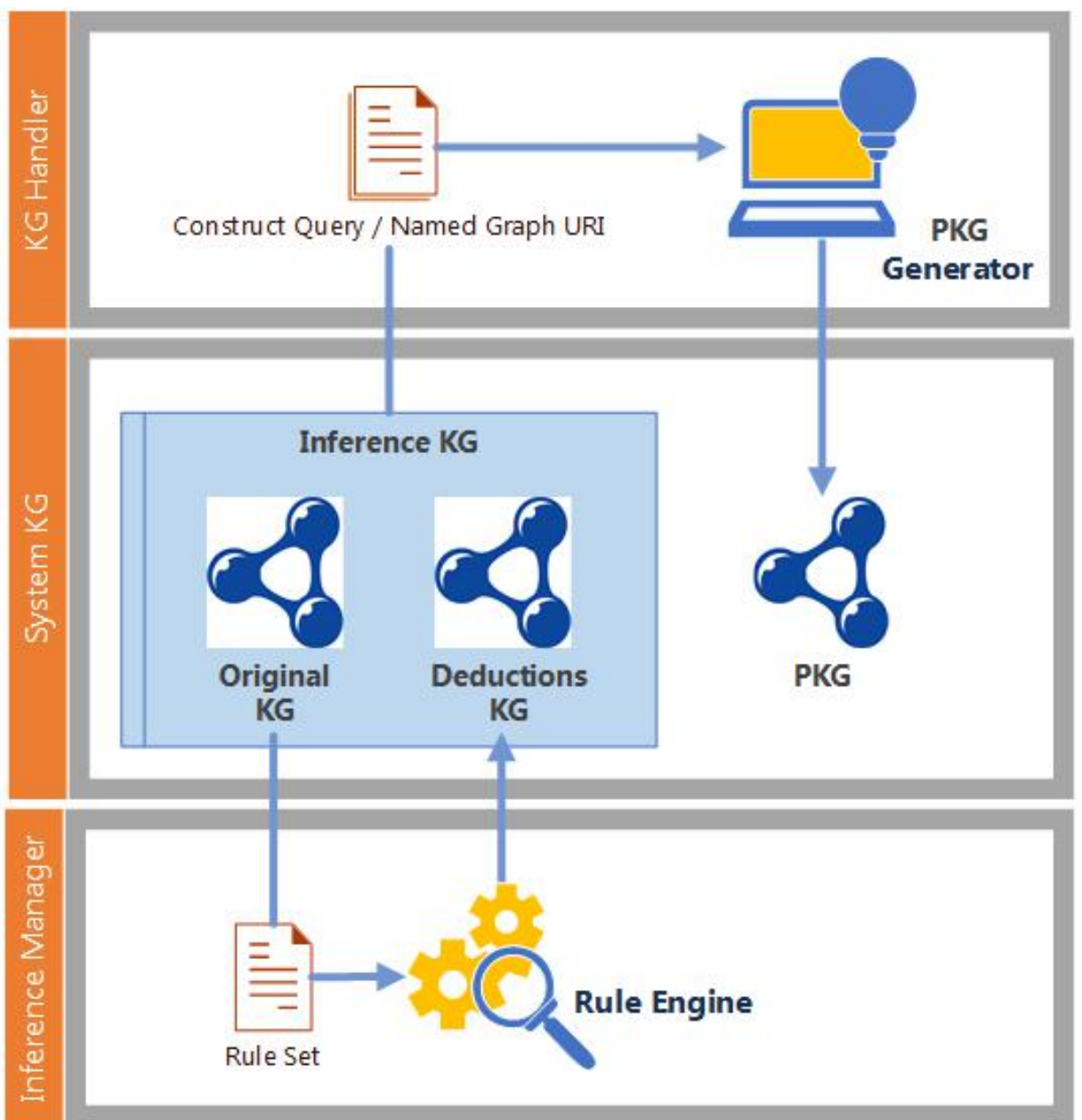

Fig. 4.1 Workflow of Private Knowledge Graph (PKG) generation

Three modules are involved: System KG, Inference Manager and KG Handler:

- System KG works as the knowledge base for the storage of information, the deducted result and the PKGs.

- Inference Manager is a module in the Model Manager, which is responsible for carrying out inference according to the defined rules.

- KG Handler is part of the Security Manager which is in charge of the PKG generation. There are two methods for the PKG Generator to create a PKG:

- Executes a SPARQL CONSTRUCT Query to the Inference KG and retrieves the demanded statements to form a PKG 
- Retrieves one or several named graphs according to the given URIs and combine them as a PKG.

Different methods are adapted according to the type of agents, which will be explained in detail in Chapter 5.

The Original KG contains an IoT-Centric ontology BATOnt and the data stored with the schema provided by the BATOnt. BATOnt stands for ontology for Building Automation Technology, which is an ontology developed by CeDInt. It defines the schema and a common vocabulary for the operations in an IoT solution. The namespace of BATOnt is defined as "bat: www.cedint.upm.es/bat.owl\#". It provides the schema and vocabulary for the data stored in the System KG. System KG provides a semantic description of all the entities involved in the IoT System.

\subsubsection{Creation of BATOnt}

The creation of BATOnt started with some core concepts and relations for system operations. The first project implemented on the basis of the platform was about smart residence monitoring. Later, with more practical implementation for projects[107], BATOnt was expanded to cover a wider range of domains, from the level of smart buildings to smart cities. Meanwhile, three predefined enumerations have been defined in BATOnt to achieve a better agreement among the developers.

In general, the projects that have been implemented on the basis of the IoT platform can be classified into three groups: home, building and campus/city. The main differences between each group is the introduction of new classes and new elements in the enumerations. Enumeration refers to a list of options that the instance of a class can be.

- The Smart home projects focus on the situation in a single flat/house, which consists of different spaces. The enumeration of space type mainly includes "Living room", "Bedroom", "Kitchen", "Bathroom", "Balcony", etc., and the devices commonly deployed are ambient sensors, HVAC control system, lighting system, and blind actuators.

- At the level of Smart building, two projects were implemented respectively for the monitoring and control of CeDInt building as well as the greenhouse of CBGP research center, UPM [108]. A new concept architecture was then introduced in BATOnt. Moreover, more space types are defined, such as "Elevator", "Stairs", "Office", "Laboratory" and "GreenHouse". 
- The projects of Smart city usually involve an amount of architectures, whose spatial information is important. As a result, new concepts are introduced for describing locations including geographical coordinates and address. New space types are added such as "Street" and "Park". A pilot project of smart street lighting has been implemented at the Campus Montegancedo, UPM [97].

BATOnt includes the definition of classes, attributions, relationships in the domain of the Internet of Things (IoT). In total, BATOnt consists of 31 concepts and 44 object properties (38 explicit ones and 6 implicit ones), which distribute in six conceptual modules: "Things", "Control", "Construction", "GeoData", "Administration" and "Person". Fig. 4.2 shows all the key classes, datatype properties (attributions) and object properties (relationships) of BATOnt.

The vocabulary provided by BATOnt is for describing not only the Things, but also the related concepts that could be involved in an IoT project implementation, which are about people, spaces, control and authorization. In general, BATOnt can be interpreted from four main perspectives:

- Things perspective, with a focus on which communication protocol is selected, how the devices are deployed and how the Things sense and actuate.

- Control perspective, with a focus on how to control the Things through applications and how to coordinate the collaboration among different Things.

- Spatial perspective, with a focus on where the Things are placed, either in the sense of geographical location or of architectural construction.

- User perspective, with a focus on the interaction of Things with human beings, i.e. who can access and control the Things and what are the characteristics of these people.

In the following sections, we will explain in detail each perspective.

\section{Things perspective}

The Things perspective includes mainly the module "Things", which is the core part of BATOnt. It gives a full description of the properties, installation and configuration of system hardware. Fig. 4.3 illustrates all the classes in the Things perspective.

As mentioned above, each local network communicates with the Central Server through a Border Router (bat:BorderRouter). Each BorderRouter is associated with a client (bat:clientId). And each Border Router exchanges information with the Things through (bat:hasTechnology) 


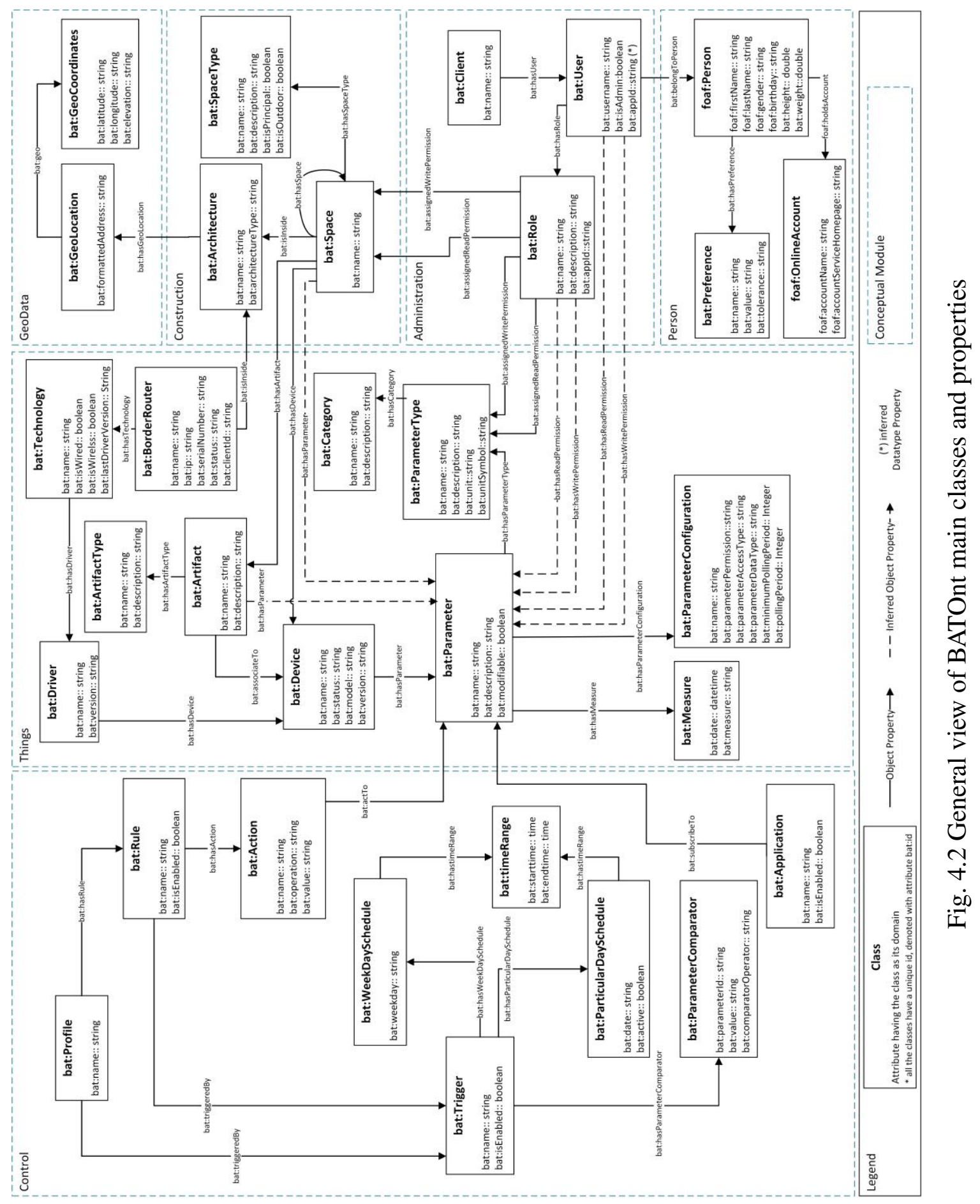




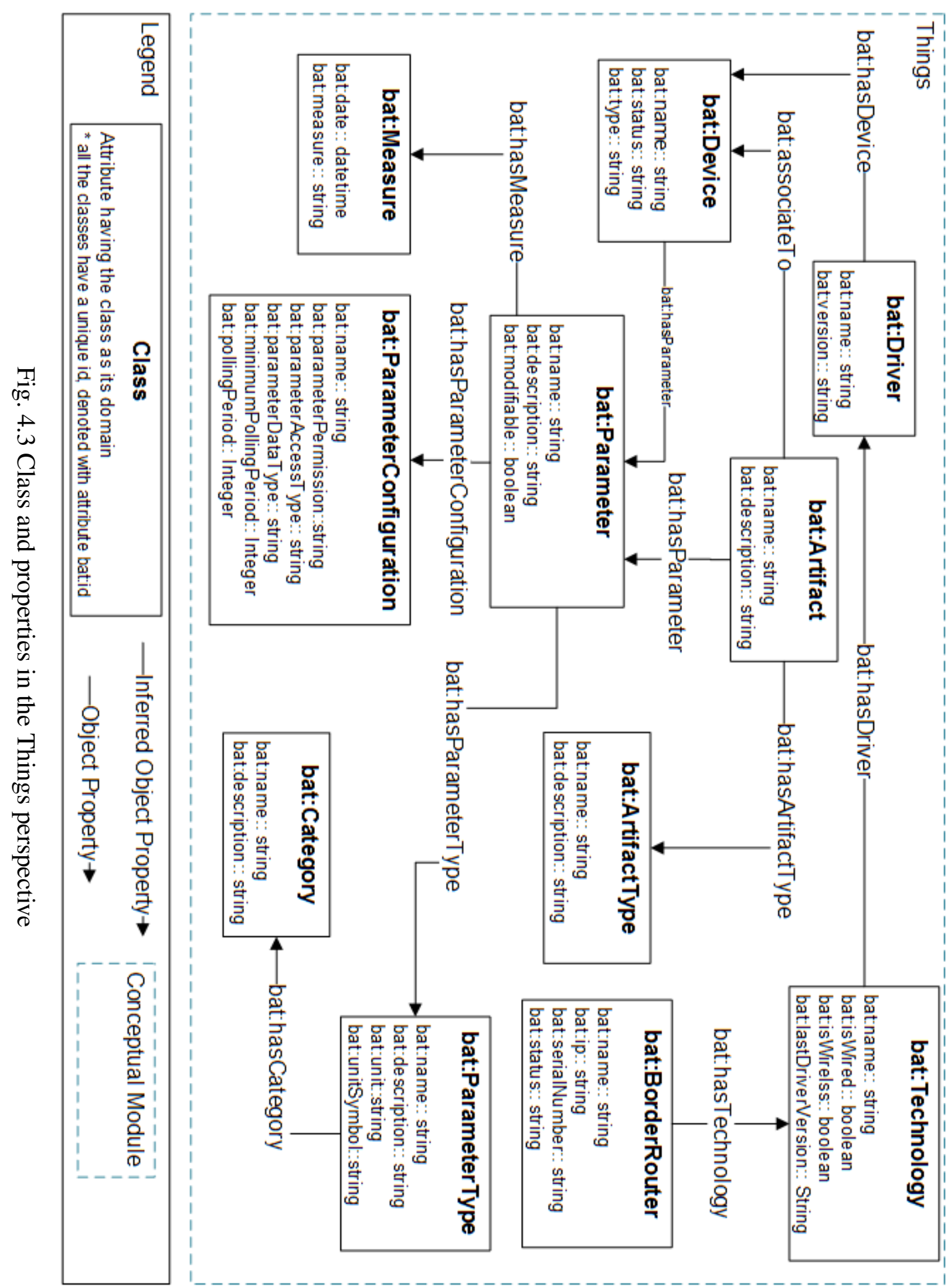


different communication protocols (bat:Technology), either through a wired/wireless network, or a mash-up of both. The features of technologies including bat:isWired and bat:isWireless can help to judge the feasibility of an installation plan. Corresponding to each technology, a driver should be provided (bat:hasDriver) as the interface between the border router and hardware devices (bat:Device). A driver is a software program that should be installed for the communication between the devices and the Border Router. It is used to interpret the messages sent from the device to the information of the parameters. Each driver must indicate its version number (bat:version). By comparing this number with the last available driver version of the technology (bat:lastDriverVersion), the system can update/upgrade driver manually or automatically.

Each driver manages (bat:hasDevice) a set of devices by interpreting them into parameters (bat:Parameter). In BATOnt, the concept device (bat:Device) is equivalent to the Things in the IoT sense. A device refers to a smart apparatus embedded with electronics, firmware, sensors and/or actuators to collect data and interact with the surroundings. Meanwhile, a device should have the capability to communicate with the border router or other devices for data exchange. The functionalities of a certain device are interpreted as different parameters respectively (bat:hasParameter) and all the data collected by the device is encapsulated as measures (bat:Measure). Measure refers to the value of a parameter at a certain moment. Each parameter has (bat:hasParameterConfiguration) its own configuration (bat:ParameterConfiguration), which contains all the necessary information for the interaction with this parameter. The parameters are classified into (bat:hasParameterType) different parameter types (bat:ParameterType), while each type may belong to (bat:hasCategory) one or several categories (bat:Category). This allows the applications to get a set of parameters in which they are interested in. Apart from device, BATOnt also has defined a similar concept artifact (bat:Artifact), which refers to those things that can not be accessed or controlled directly. The data related to an artifact should be retrieved and collected by using devices. For instance, the open/close status of a door (artifact) needs to be detected by a door sensor (device). Each artifact is also denoted with (bat:hasArtifactType) a type (bat:ArtifactType) for classification and further comparisons.

The key difference between a device and an artifact is that a device is connected to a network and can be accessed in a remote way, while an artifact is not connected into a network. The status of the artifact can be obtained by associate it to a device. An object can be regarded as either a device or an artifact in different situations. For instance, if a light actuator is connected to the Border Router and can be switched on/off by sending the command, this light is regarded as a device. However, if a light is plugged into a smart plug 
and its status is controlled by switching on/off the plug, then this light is regarded as an artifact, which associates to this plug and the plug is regarded as a device.

\section{Control perspective}

The control perspective includes mainly the module "Control". It describes the system work flow from the software perspective, i.e. how the Things work collaboratively to achieve certain missions. Fig. 4.4 illustrates all the classes in the control perspective.

Users can define profiles (bat:Profile) and rules (bat:Rule) so that a series of operations can be executed simultaneously under certain scenarios. Each profile is configured with (bat:hasRule) a set of rules, and each rule is associated with (bat:hasAction) a set of actions (bat:Action). An action takes effect to (bat:actTo) a parameter, such as set it with a new value or increase/decrease its current value. Each profile and each rule can be activated (bat:triggeredBy) when certain conditions (bat:Trigger) are satisfied. There are three forms to configure a trigger:

- Time intervals (bat:timeRange) of some fixed days of a week (bat:WeekDaySchedule)

- Time intervals of some specific dates (bat:ParticularDaySchedule)

- When the values of some parameters meet certain conditions (bat:ParameterComparator)

When a rule is activated, the associated actions are executed. When a profile is activated, the actions defined in all its associated rules are executed.

In the platform, to satisfy different client demands, different applications (bat:Application) are developed. Each application can subscribe to (bat:subscribeTo) a set of parameters so as that it can obtain the value of the parameters.

\section{Spatial perspective}

The spatial perspective includes two modules, "Construction" and "GeoData". It describes the location of the Things and the architectural construction where the Things are placed. Fig. 4.5 illustrates all the classes in the spatial perspective.

The border router should indicate its location, i.e. the architecture (bat:Architecture) where it is placed (bat:isInside). Each architecture may be divided, either physically or logically, into (bat:isInside) several spaces (bat:Space) where the devices and artifacts are placed (bat:hasDevice, bat:hasArtifact). In some cases, a space may contain (bat:hasSpace) several minor spaces inside. Each space is annotated with (bat:hasSpaceType) a type (bat:SpaceType) so that customised services can be provided accordingly. 


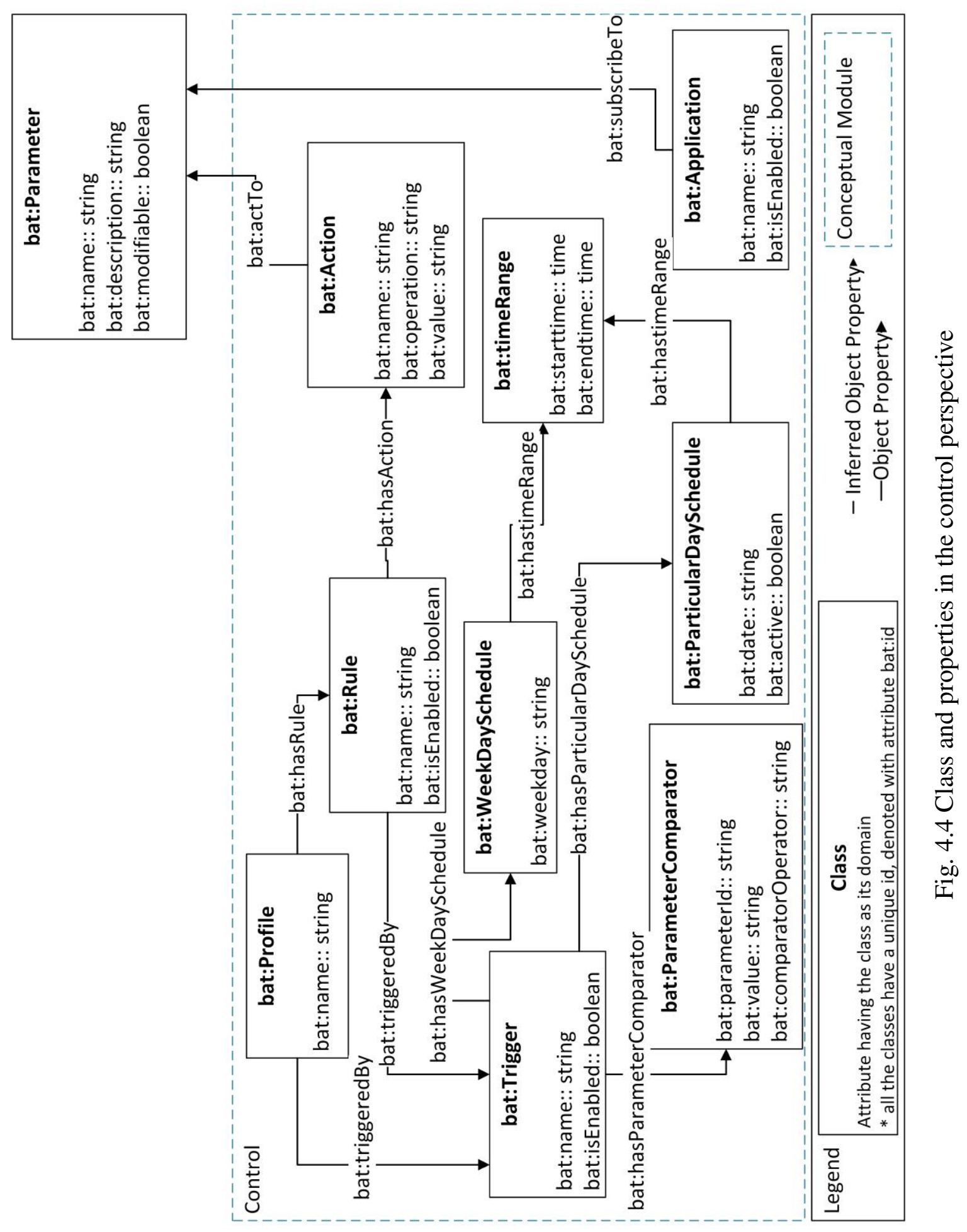




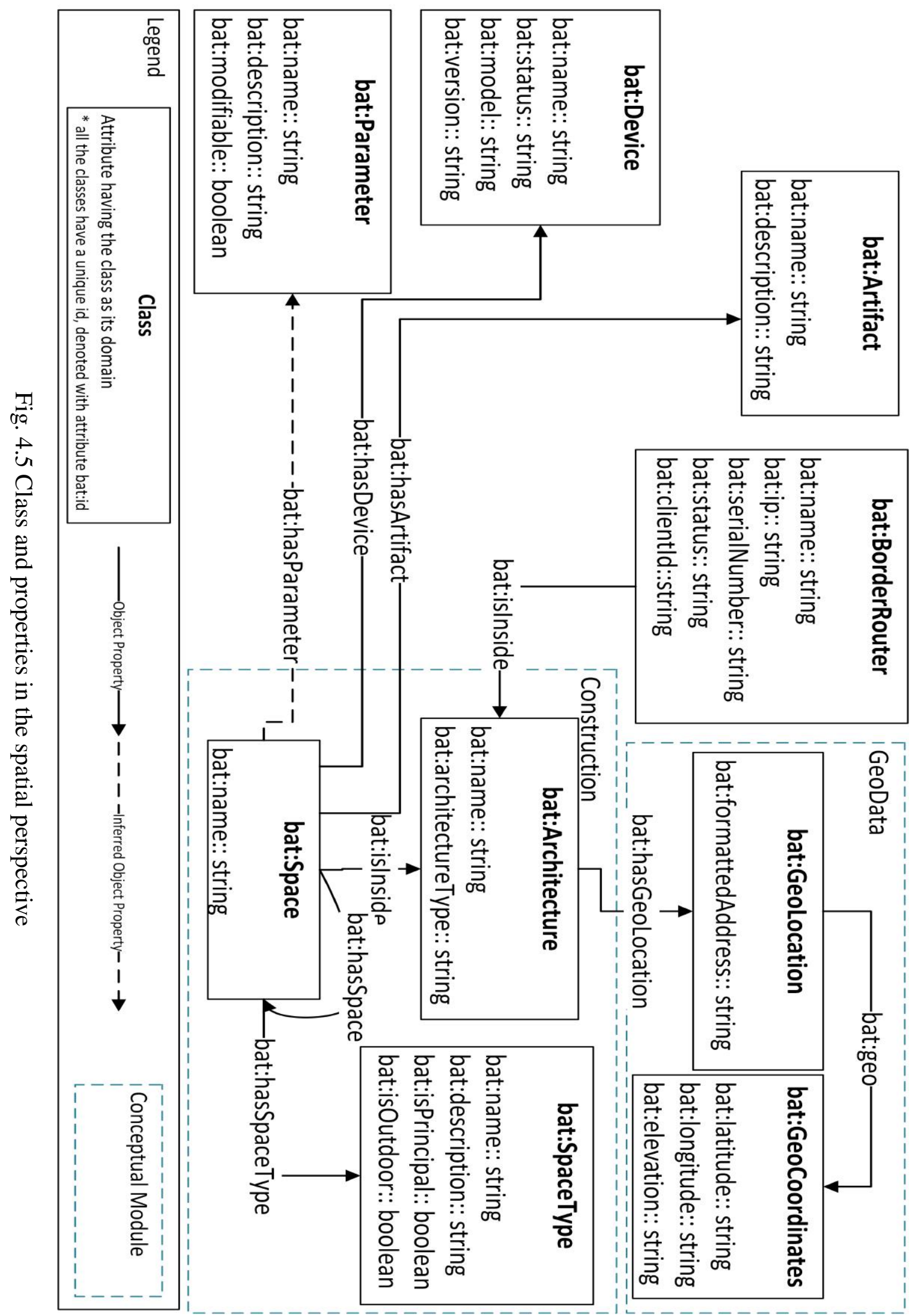


One common way to display IoT data is showing the location-based overview of the Things deployment and information [109] in a map view. By knowing the geographic coordinates of a Thing, it can be marked out precisely on a map. Considering the fact that currently there are various map providers in the market, such as OpenStreetMap [110] [111], MapBox [112], Google Map API [113] and schema.org[114], a class has been defined to represent the geographical location (bat:GeoLocation) of the architecture (bat:hasGeoLocation), indicating (bat:geo) its geographic coordinates (bat:GeoCoordinates), instead of choosing a specific vocabulary provided by a unique tool. As a result, developers can take advantage of the information provided by System KG, while choosing their preferred map service.

\section{User perspective}

The user perspective contains mainly the modules "Administration" and "Person". The module Administration describes the authorization control mechanism of user accounts and the module Person describes the human beings who hold the accounts. Fig. 4.6 illustrates all the classes in the user perspective.

In this module, part of the widely recognized ontology FOAF [78] has been imported, with the namespace "foaf:http://xmlns.com/foaf/0.1/". An entity, which can be a person, a family or an organization, becomes a client (bat:Client) after having registered in the platform. Client is a way to group a set of users who are sharing devices and spaces. Each client has (bat:hasUser) a list of users (bat:User). A user refers to an account that is hold by (bat:belongToPerson) a person (foaf:Person) to access the system. In the developed IoT platform, the first user of the registered client is regarded as the administrator(bat:isAdmin is true). Each client has one and only one user which is the administrator. This user has the right to change the space layout configuration, install new devices, add/delete artifacts, etc. While all the other users can only have access to the information that they are permitted to $\mathrm{read} / \mathrm{write}$.

In the platform, the access control to the Things is carried out following a role-based approach. Each role (bat:Role) is assigned with permission to read (bat:assignedReadPermission) or write (bat:assignedWritePermission) to a list of parameter types (PT) and spaces (S). A role is authorized to read (bat:hasReadPermission) a/o write (bat:hasWritePermission) all the parameters that satisfy both characteristics, i.e. the set $\{\mathrm{P} \mid \mathrm{P}$ bat:hasParameterType $\mathrm{PT}\} \cap\{\mathrm{P} \mid \mathrm{S}$ bat:hasParameter $\mathrm{P}\}$.

Meanwhile, a user can read (bat:hasReadPermission) a/o write(bat:hasWritePermission) all the parameters of its roles (bat:hasRole).

In the case that a person holds several user accounts, he can share his profiling (foaf:gender, bat:height, etc.) and preferences (bat:Preference) among different systems, which can be 


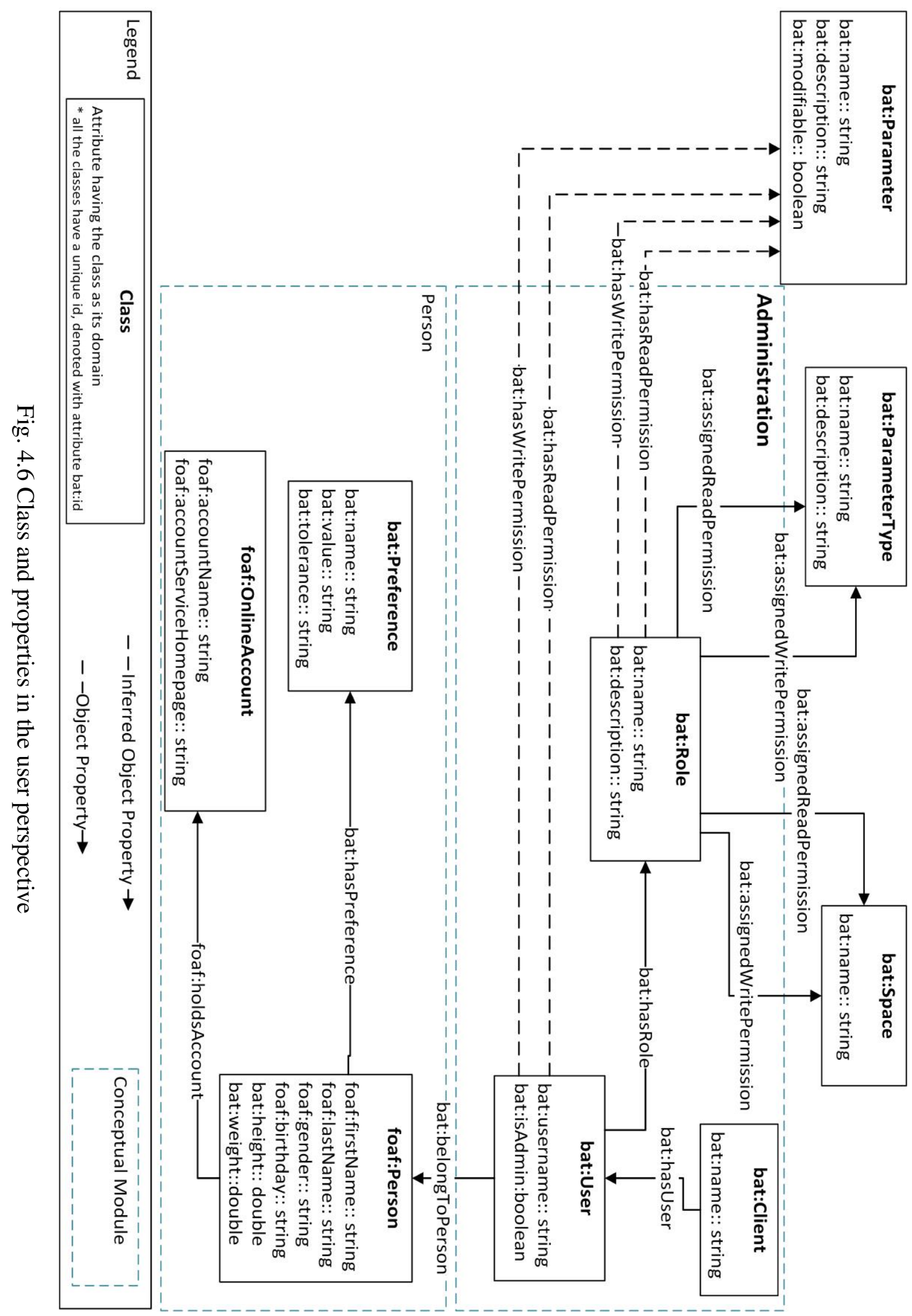


taken as reference for decision making. Regarding social network activities, by introducing the social media accounts (foaf:OnlineAccount) that a person holds (foaf:holdsAccount), the relations between this person and others can be obtained.

\section{Enumerations}

Currently, BATOnt provides three predefined enumerations for bat:ParameterType, bat:SpaceType and bat:Category. By doing this, the users of BATOnt can choose from a limited set of options, which makes it easier to reach consensus and compare across different domains. The enumerations have been defined on the basis of different IoT project. The lists of options in the enumerations are not limited, while new elements can be added on requirement. A list of all the enumerations defined in BATOnt has been attached in Appendix A.

In the enumeration for bat:Category, five individuals have been defined, which are: "Energy", "Illumination", "Temperature", "AmbientCondition" and "Administration". Listing 4.1 shows the definition of category "AmbientCondition".

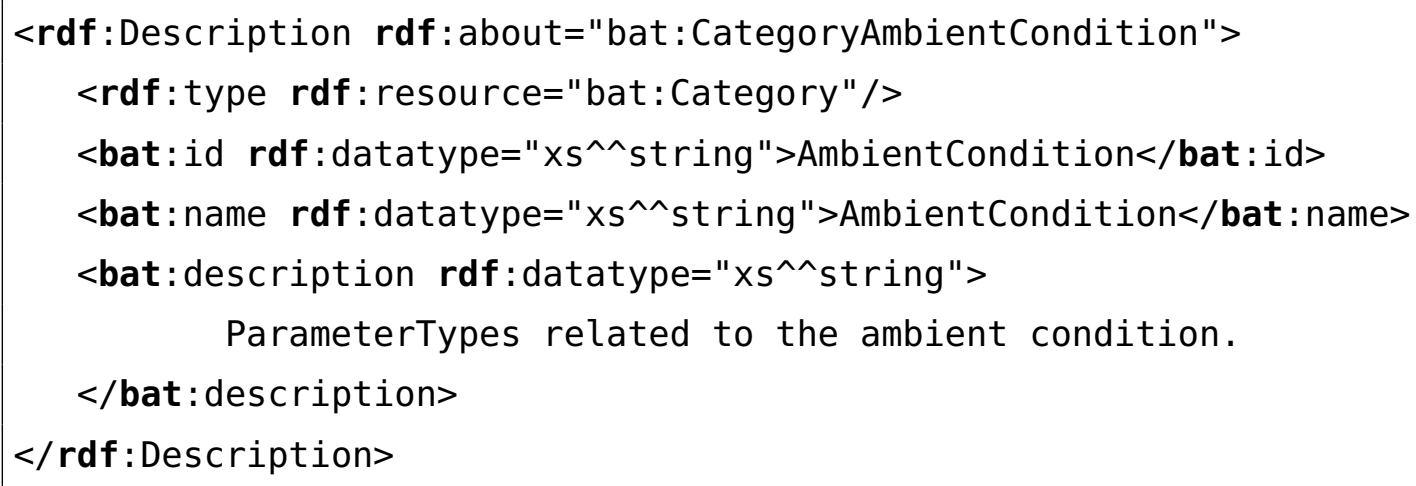

Listing 4.1 Definition of category Ambient Condition

In the enumeration for bat:ParameterType, twenty individuals have been defined for bat:ParameterType, which are: "DimmingActuatorPercentage", "BlindActuatorPercentage", "BlindActuatorPercentageInverted", "IlluminationLevel", "SolarAzimuthAngle", "SolarElevationAngle", "RainStatus", "WindSppeKMPerHour", "BatteryVoltage", "Presence", "Administration", "Humidity", "TemperatureCelsius", "TemperatureFahrenheit", "Voltage", "Current", "RealPower", "ApparentPower", "RealEnergy" and "OnOff". For each parameter type, the corresponding name, description, unit and unitySymbol are defined. Listing 4.2 shows the definition of parameter type "Humidity". 


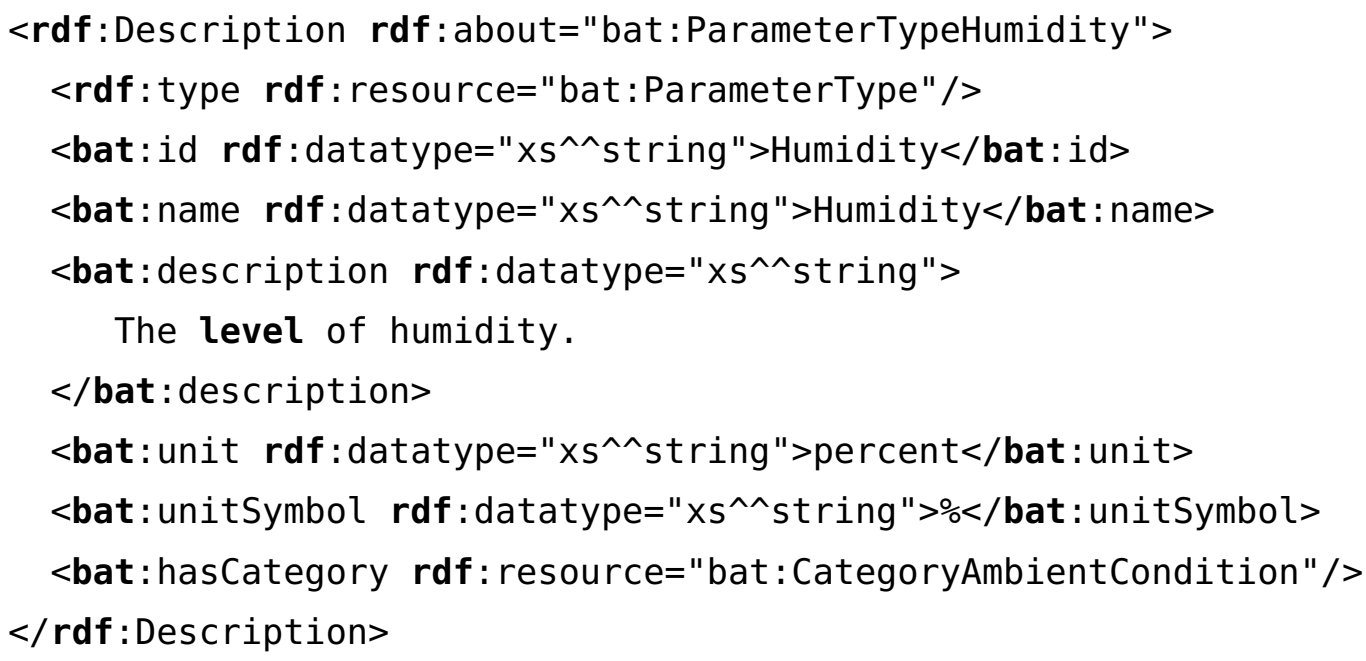

Listing 4.2 Definition of parameter type Humidity

In the enumeration for bat:SpaceType, 36 individuals have been defined, which are: "Flat", "Chalet", "Park", "Street", "Office", "Showroom", "Warehouse", "MeetingRoom", "WaitingRoom", "ConferenceHall", "ServerRoom", "ControlRoom", "Elevator", "Stairs", "LivingRoom", "Kitchen", "StudyRoom", "PantryRoom", "StorageRoom", "LaundryRoom", "Bathroom", "Bedroom", "Porch", "Terrace", "Balcony", "Garden", "Patio", "Laboratory", "Greenhouse", "InternalParking", "ExternalParking", "Entryway", "Vestibulo", "Corridor", "SwimmingPool", "Path". Listing 4.3 shows the definition of space type "LivingRoom".

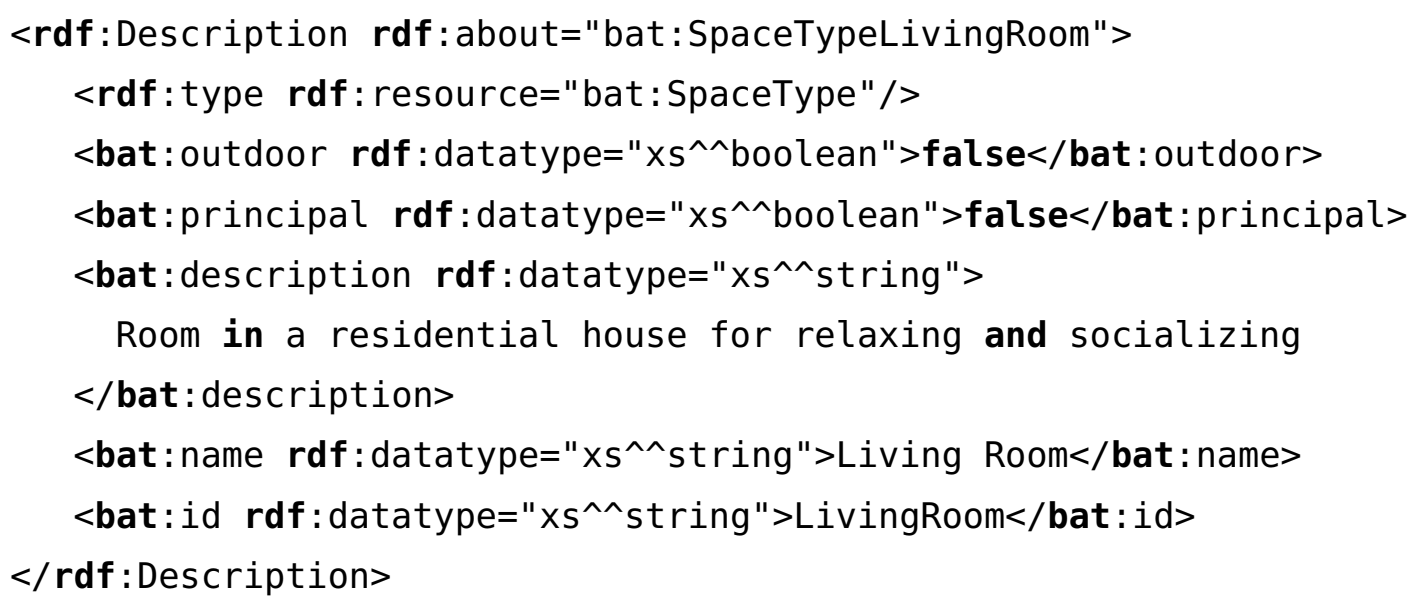

Listing 4.3 Definition of space type LivingRoom

\subsubsection{Dataset structure of System KG}

In the System KG, the data is stored with the schema provided by the BATOnt, which describes the actual facts in the system. By using the syntax and terminologies provided by 
BATOnt, the data in $\mathrm{KG}$ expresses the information in a machine-understandable way. Each individual is an instance of a class in BATOnt and the relations between those individuals comply with the definition in BATOnt. In short, BATOnt and the data form the System KG, which work as a whole to provide the necessary knowledge in the platform, where BATOnt defines the structure and the vocabulary while the data stores the information for query and inference.

The System KG is stored in a dataset consisting of a number of name graphs. The knowledge graph for a client consists of two parts:

- A named graph represented by a URI "batmp.cedint.upm.es/BATOnt/\{clientID $\}$ ”

- The deductions model of this named graph, which is stored in another named graph, which is represented by URI "batmp.cedint.upm.es/BATOnt/inf/\{clientID $\}$ "

where clientID refers to the identifier of the client. The combination of these two named graphs forms the entire knowledge graph for this client, which will be denoted as "NGclientID" in the following parts. Apart from the named graph for each client, System KG contains one special named graph, "NG-Basic", which stores all the classes, relations and the predefined enumerations of BATOnt. Fig. 4.7 illustrates the structure of System KG.

The illustrated System KG DataSet consists of three named graphs, NG-Basic and two named graphs for clients, NG-Client0001 and NG-Client0002. The classes bat:Space, bat:Device, bat:Parameter, bat:Role, bat:ParameterType and bat:User are all stored in NGBasis as part of BATOnt. I addition, the individual bat:TemperaturCelsius is a predefined parameter type. Therefore, although it is an individual of the class bat:ParameterType, it also parts of the BATOnt, as one element of the bat:ParameterType enumeration. NG-Client0001 contains all the individuals that belong to client 0001, each of which is the instance of a class in the BATOnt. The ex:SetTemperatureSW, ex:SWDaikin, ex:officeSW, ex:RoleSW, ex:user and ex:user2 are all the instances of the corresponding classes and belong to the client 0001 . Therefore, they are stored in NG-Client0001 as individuals.

\subsubsection{SPARQL queries}

By making SPARQL queries, a certain type of parameters or devices can be obtained. Listing 4.4 shows a SPARQL query which gets all the unmodifiable temperature parameters. By getting this list, the id of all the temperature sensors which measures in degree Celsius can be obtained. 


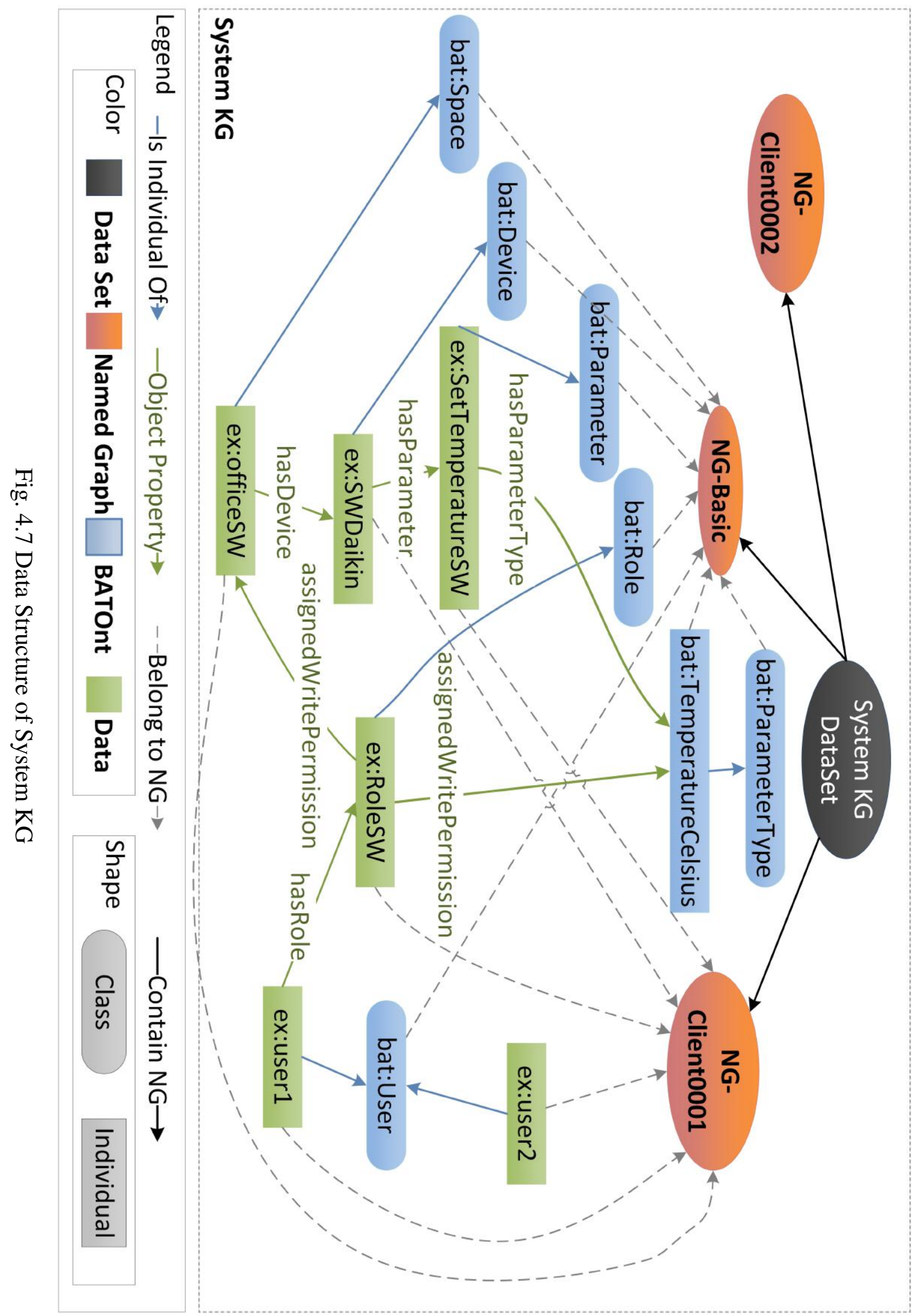




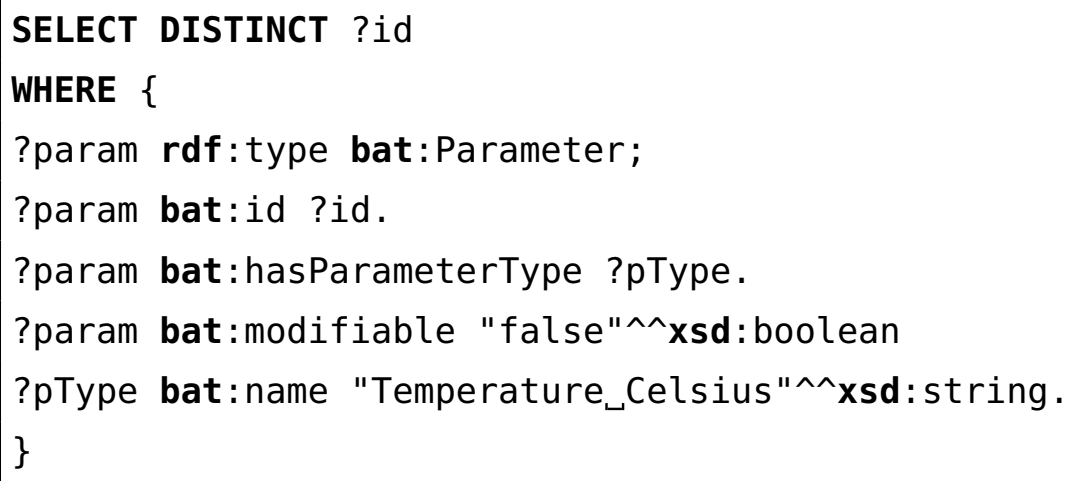

Listing 4.4 SPARQL query for temperature parameters

Listing 4.5 shows the query for obtaining all the energy-related devices. This SPARQL query is for retrieving the Ids of a list of devices, each of which has at least one parameter that has a parameter type which belongs to the category "Energy".

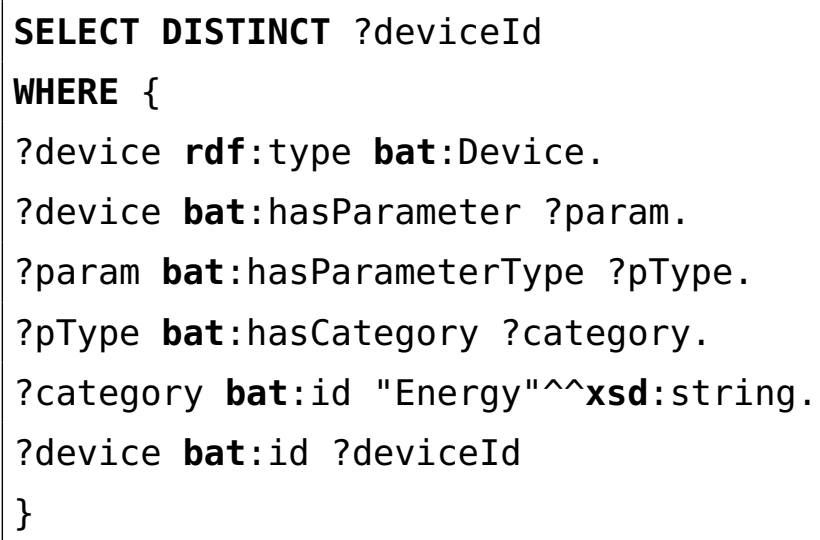

Listing 4.5 SPARQL Query for all energy-related devices

BATOnt provides a vocabulary to give semantic description of all the entities involved in the IoT domain from four perspectives: Things perspective, Control perspective, Spatial perspective and User perspective. This ontology is scalable and allow the inclusion of new classes. According to different use cases, the ontology was being evolved. The ontology provides a schema for the data stored in a open and standard IoT platform. Apart from the object properties, the ontology also contains implicit object properties, which should be obtained by the inference. The next section will explain in detail about the process of inference and the definition of rules. 


\subsection{Inference and rules definition}

In a knowledge graph, apart from the facts that are already explicitly stated in the knowledge base, there are still information that can be retrieved following specific logic. The process is called inference and should be carried out by a rule engine. The logic is stated semantically in the form of rules. In this thesis, Jena Generic rule reasoner is used as the system rule engine, which supports user-defined rules.

\subsubsection{Objective}

In the platform, a friendly user interface is provided for the administrator of a client to manage users, roles, space, devices and applications. However, the information which interests more is the implicit ones such as what parameters are located in a specific space, which parameters can be accessed by a certain user. These implicit relationships are very abstract for a normal user, but essential for the system decision-making. With the large quantity of entities in the System KG, it is impossible to set these relationships one by one. As a result, inference has been adapted in order to retrieve the implied relationships among the entities.

In this section, a subset of BATOnt will be illustrated, which includes all the concepts involved in the process of access control. Fig. 4.8 shows the concepts and relationships in the subset.

Fig. 4.9 shows the inferred object properties. In the case of the platform, two kinds of implicit information are obtained. The first kind is the relationships between parameters and spaces/artifacts, which is obtained by applying the fact rules. The second is the authorization relationships among users, roles and parameters, which is obtained by applying the policy rules.

\subsubsection{Rule set definition}

A rule represents the logic to follow to get the implied or add-value information. Jena provides a Generic rule reasoner which supports user defined rules, which should be represented in the form of Jena Rule. Jena Rule is designed to be used only by the inference engine in the Jena framework. Each rule consists of a rule name, a set of conditions and a set of results. Both of the conditions and results are represented in the form of triples. Meanwhile, Jena Rule also has a set of builtin primitives such as remove, noValue and sum for advanced functions [115]. The rules in the platform can be general classified into two types: fact rules and policy rules. 


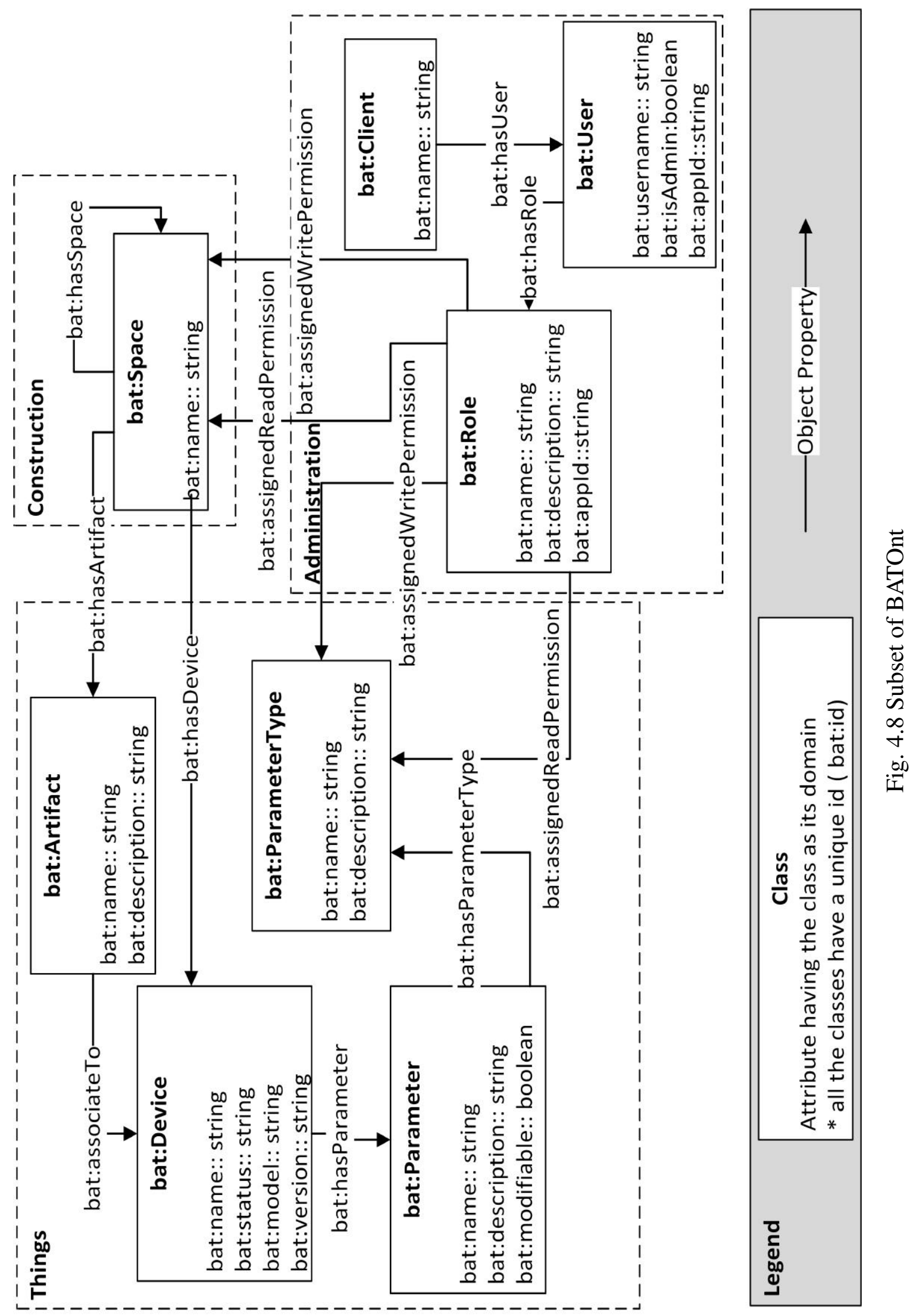




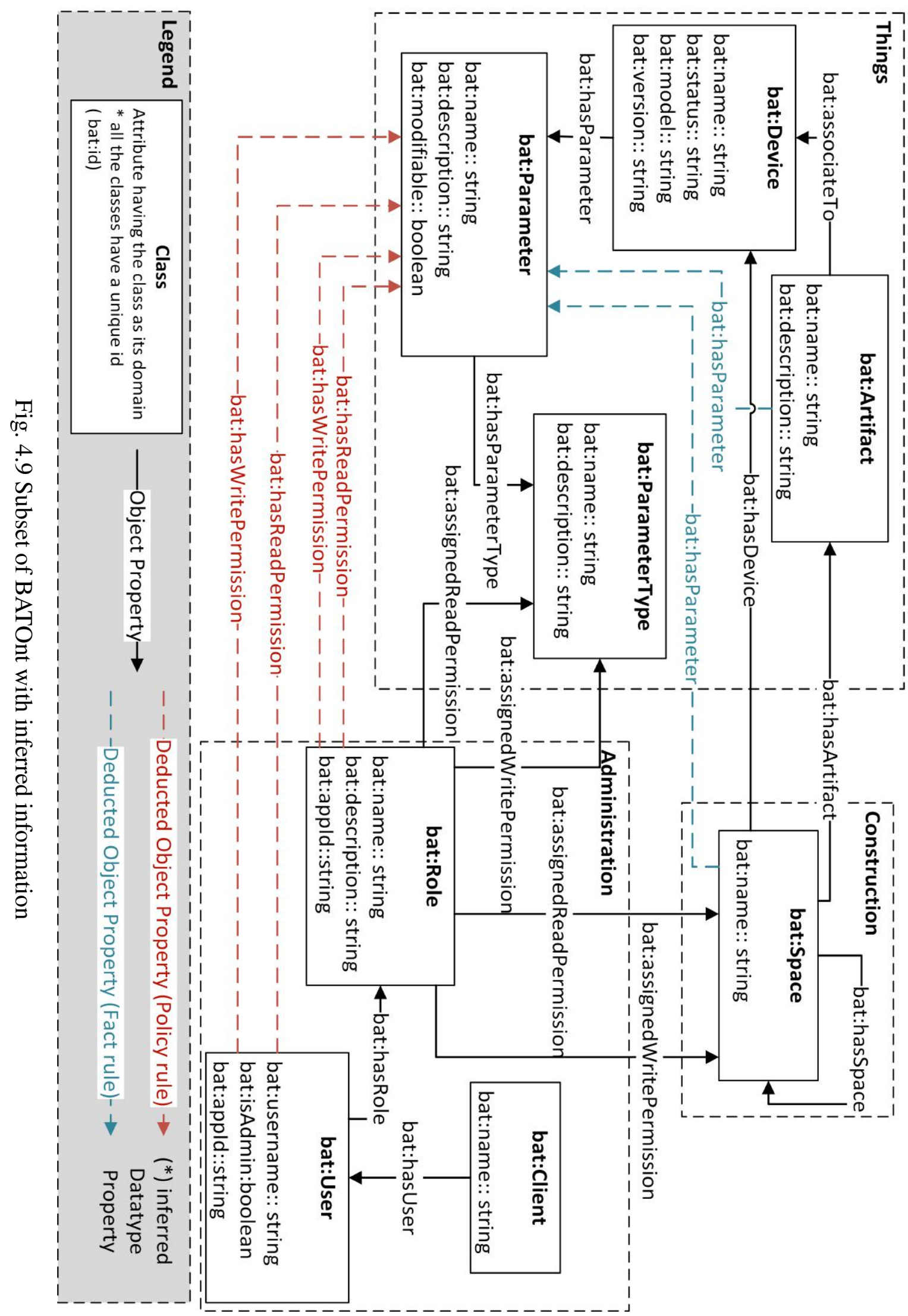




\section{Fact rules}

Fact rules state the substantial facts that can be observed. In the platform, the administrator can configure the spaces, devices and artifacts through a friendly user interface. And three fact rules are defined to discover the implicit relationships among these spaces, devices and artifact. The rules are enumerated in Listing 4.6 in the form of Jena Rule:

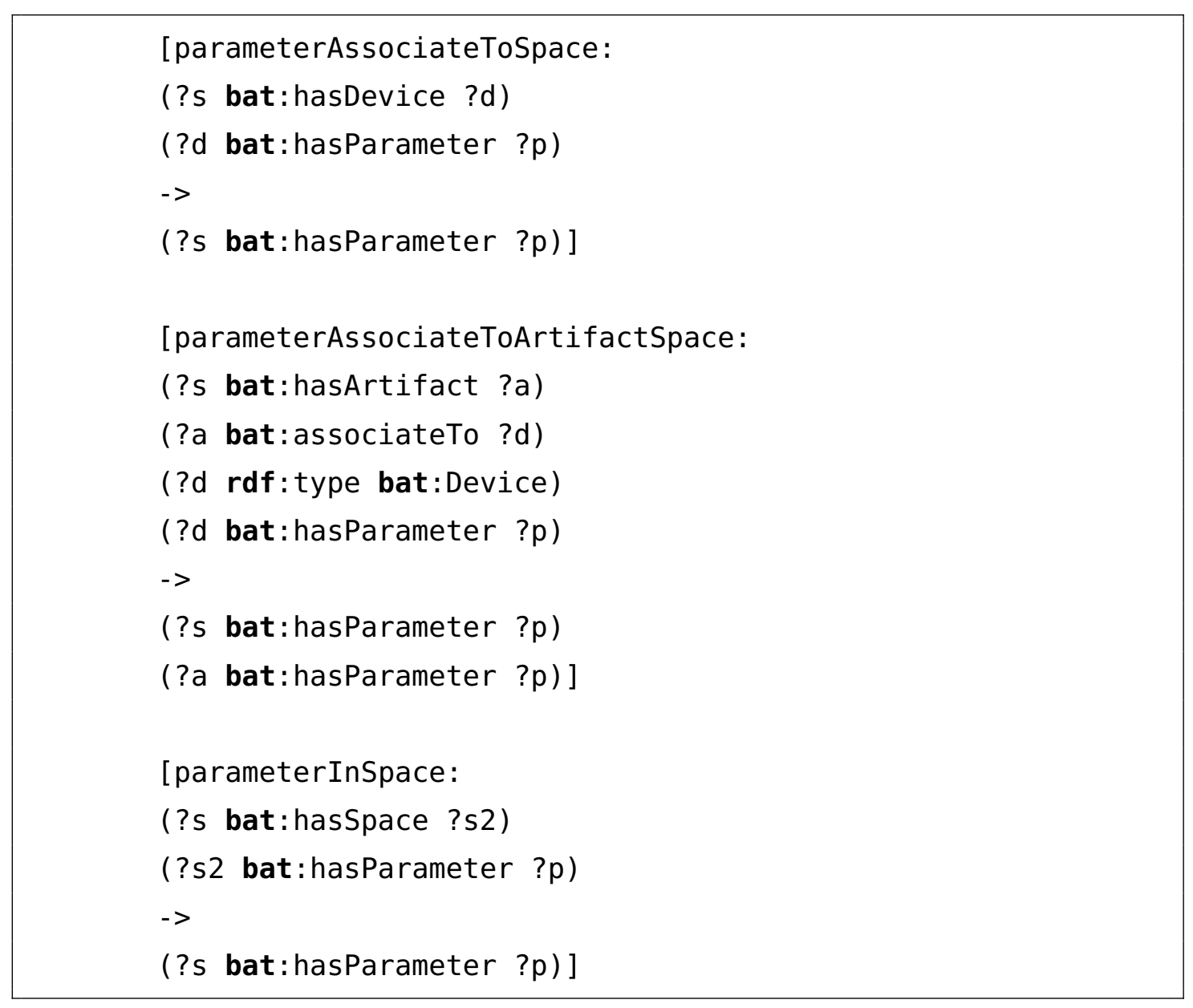

Listing 4.6 Formalization of rules in Jena

- Rule parameterAssociateToSpace states that if a device belongs to a space, then all the parameters of this device will also belong to this space. Each client has a list of spaces, each space has a list of devices inside and each device has a list of parameters. If a parameter $\mathrm{P}$ belongs to a device $\mathrm{D}$ while $\mathrm{D}$ locates inside a space $\mathrm{S}$, then $\mathrm{P}$ is inside $\mathrm{S}$.

- Rule parameterInInnerSpace states that if a space S1 has other spaces inside, then all the parameters belonging to these inner spaces will also belong to S1 
- Rule parameterAssociateToArtifactSpace states that if an artifact belongs to a space and associates to a device at the same time, all the parameters of this device belong to this artifact as well as this space.

\section{Policy rules}

Policy rules state the principles or protocols to follow for making decisions. Policy rules are defined to implement the trust and privacy policies which specify the information can be accessed and the device can be controlled by a certain user. Considering that the platform is a multi-technology platform, the authorization of a user to perform an operation is represented in the form of Read/Write permission between the user and the parameter. The authorization mechanism in the platform is carried out following a combination of role-based and attributebased approach. The access control of users to the Things is configured in two steps:

[1] Decide if a role has permission to read or write a parameter according to the type and location of the parameter.

[2] Decide if a user has permission to read or write a parameter according to his roles.

Meanwhile, a rule is provided to decide the authorized applications of a user according to his roles. In the following part, we will give a detailed description of the mentioned rules.

\section{Rules for Role-Parameter permissions}

The first user of each client is the administrator of this client and only the administrator is allowed to modify the configuration of user, space, application, profiles and the devices of the system. An administrator is able to define new roles and assign each role with read and/or write permission to a set of parameter types $\left\{\mathrm{PT}_{1}, \mathrm{PT}_{2}, \ldots, \mathrm{PT}_{m}\right\}$ and spaces $\left\{\mathrm{S}_{1}, \mathrm{~S}_{2}, \ldots, \mathrm{S}_{n}\right\}$. The permission relationship between roles and parameters can be decided by following the logic shown in Table 4.1 .

- A role will be authorized to read and/or write all the parameters that satisfy both characteristics, i.e. the subset $\{\mathrm{P} \mid \mathrm{P}$ bat:hasParameterType $\mathrm{PT}\} \cap\{\mathrm{P} \mid \mathrm{S}$ bat:hasParameter $\mathrm{P}\}$.

- If a role has neither Read nor Write permission to a parameter, then the role has no right to access this parameter.

Listing 4.7 and Listing 4.8 show the policy rules for Role-Parameter permissions in the form of Jena rules. 
Listing 4.7 shows the rules that are applicable to the roles that have no write access to the parameter type and/or the space. In this case, the role is only permitted to read the parameters that satisfy both characteristics:

- Rule RoleReadRead states that if a role is assigned read permission to a space and a parameter type, then it has read permission to the parameter which has this parameter type and is in this space.

- Rule RoleReadWrite states that if a role is assigned read permission to a space and write permission to a parameter type, then it has read permission to the parameter which has this parameter type and is in this space.

- Rule RoleWriteRead states that if a role is assigned write permission to a space and read permission to a parameter type, then it has read permission to the parameter which has this parameter type and is in this space.

\begin{tabular}{|c|c|c|c|c|}
\hline \multicolumn{2}{|c|}{ Permission assigned to $R$} & \multirow{2}{*}{$\begin{array}{l}\text { Parameter } \\
\text { modifiable }\end{array}$} & \multirow{2}{*}{ Common conditions } & \multirow{2}{*}{$\begin{array}{l}\text { Result Permis- } \\
\text { sion of } R \text { to } P\end{array}$} \\
\hline PT & $\mathbf{S}$ & & & \\
\hline Read & Read & Yes/No & \multirow{5}{*}{$\begin{array}{c}\mathrm{P} \text { hasParameterType PT } \\
\mathrm{S} \text { hasParameter } \mathrm{P}\end{array}$} & Read \\
\hline Read & Write & Yes/No & & Read \\
\hline Write & Read & Yes/No & & Read \\
\hline Write & Write & No & & Read \\
\hline Write & Write & Yes & & Write \\
\hline
\end{tabular}

Table 4.1 Rules for deciding permissions of roles to parameters 
[RoleReadRead:

(?r rdf:type bat:Role)

(?s rdf:type bat:Space)

(?pt rdf: type bat:ParameterType)

(?p rdf:type bat:Parameter)

(?s bat: hasParameter ?p)

(?p bat:hasParameterType ?pt)

(?r bat:assignedReadPermission ?s)

(?r bat:assignedReadPermission ?pt)

$->$

( ?r bat: hasReadPermission ?p)]

[RoleReadWrite:

(?r rdf:type bat:Role)

(?s rdf:type bat:Space)

(?pt rdf:type bat:ParameterType)

(?p rdf:type bat:Parameter)

( ?s bat: hasParameter ?p)

(?p bat:hasParameterType ?pt)

(?r bat:assignedReadPermission ?s)

(?r bat:assignedWritePermission ?pt)

$->$

( ?r bat: hasReadPermission ?p)]

[RoleWriteRead:

(?r rdf:type bat:Role)

(?s rdf:type bat:Space)

(?pt rdf: type bat:ParameterType)

(?p rdf:type bat:Parameter)

(?s bat: hasParameter ?p)

(?p bat:hasParameterType ?pt)

(?r bat:assignedWritePermission ?s)

(?r bat:assignedReadPermission ?pt)

$->$

( ?r bat: hasReadPermission ?p)]

Listing 4.7 Formalization of rules in Jena 
If a role has write access to both of the parameter type and space of a parameter, the final Role-Parameter permission should be decided by revising the attribute "modifiable" of the parameter. Listing 4.8 shows the formalization of rules in the form of Jena rules for this case:

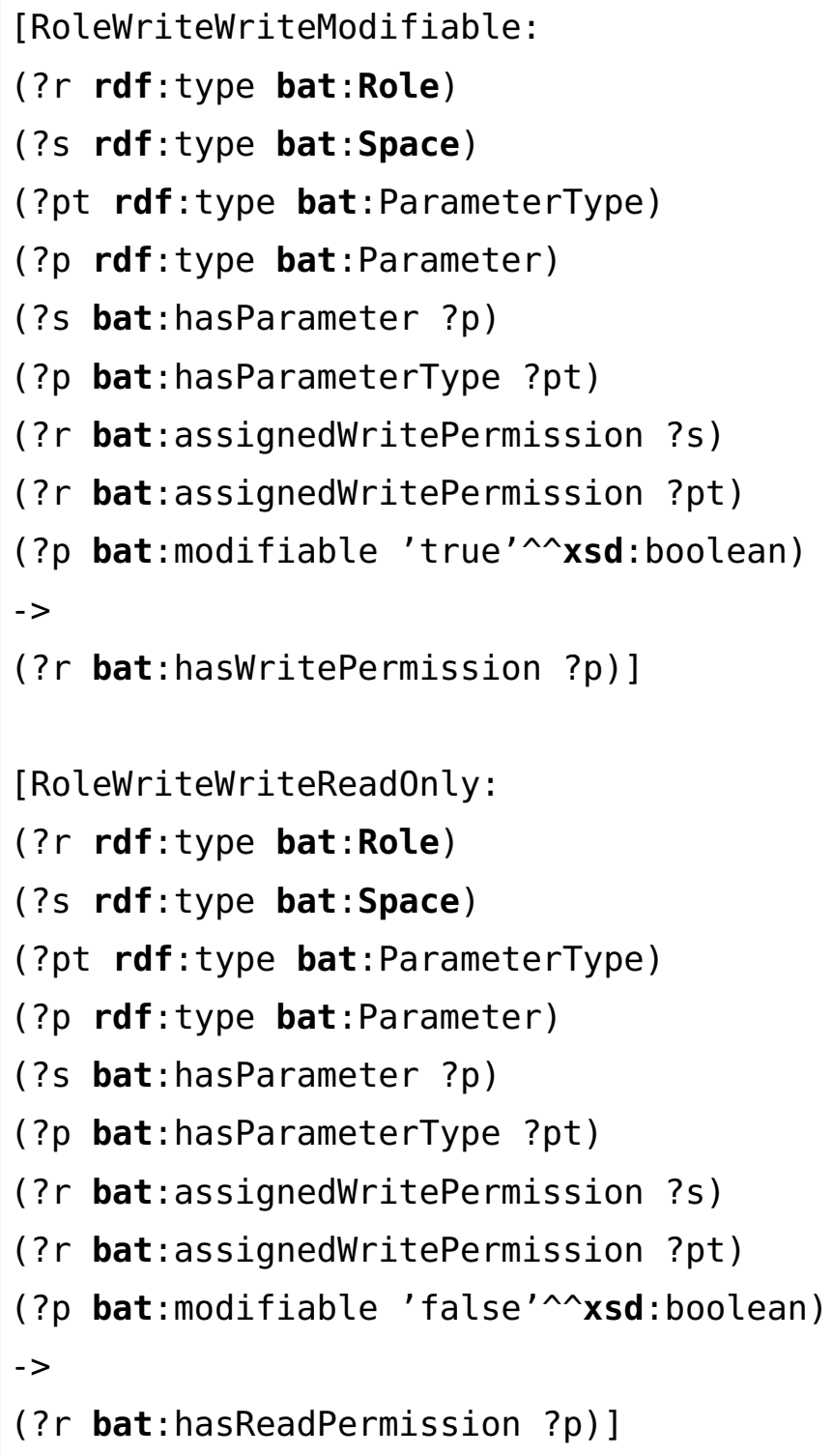

Listing 4.8 Formalization of rules in Jena

- Rule RoleWriteWriteModifiable states that if a role is assigned write permission to a space and a parameter type, then it has write permission to the parameter which has this parameter type and is in this space and is modifiable. 
- Rule RoleWriteWriteReadOnly states that if a role is assigned write permission to a space and a parameter type, then it has read permission to the parameter which has this parameter type and is in this space but is not modifiable.

\section{Rules for User-Parameter permissions}

In the case that a user has several roles, the user will have all the access rights to parameters that his roles have. Table 4.2 shows the logic for deciding the permission relationship between users and parameters.

\begin{tabular}{|c|c|c|c|}
\hline \multicolumn{2}{|c|}{ Permission Type of Rx to access P } & Common conditions & $\begin{array}{c}\text { Result Permission } \\
\text { of U to P }\end{array}$ \\
\cline { 1 - 2 } R1 & R2 & & Read \\
Read & Read & U hasrole R1 & Write \\
Write & Write & U hasRole R2 & Write \\
Write & Read & & Write \\
\hline
\end{tabular}

Table 4.2 Rules for deciding permissions of users to parameters

Listing 4.9 shows the formalization of rules in the form of Jena rules:

- Rule UserRead states that if one of the user's roles can read a parameter, then this user is permitted to read this parameter.

- Rule UserWrite states that if one of the user's roles can modify (write) a parameter, then this user is permitted to modify (write) this parameter.

- Rule UserReadWrite deals with the case that a user has several roles, some of the roles may have Read permission to a parameter while some have Write permission to the same parameter. As a result, the redundant relationship bat:hasReadPermission between this user and this parameter is removed by this rule, as it is supposed that the permission Write is superior than Read in the sense of access control. 


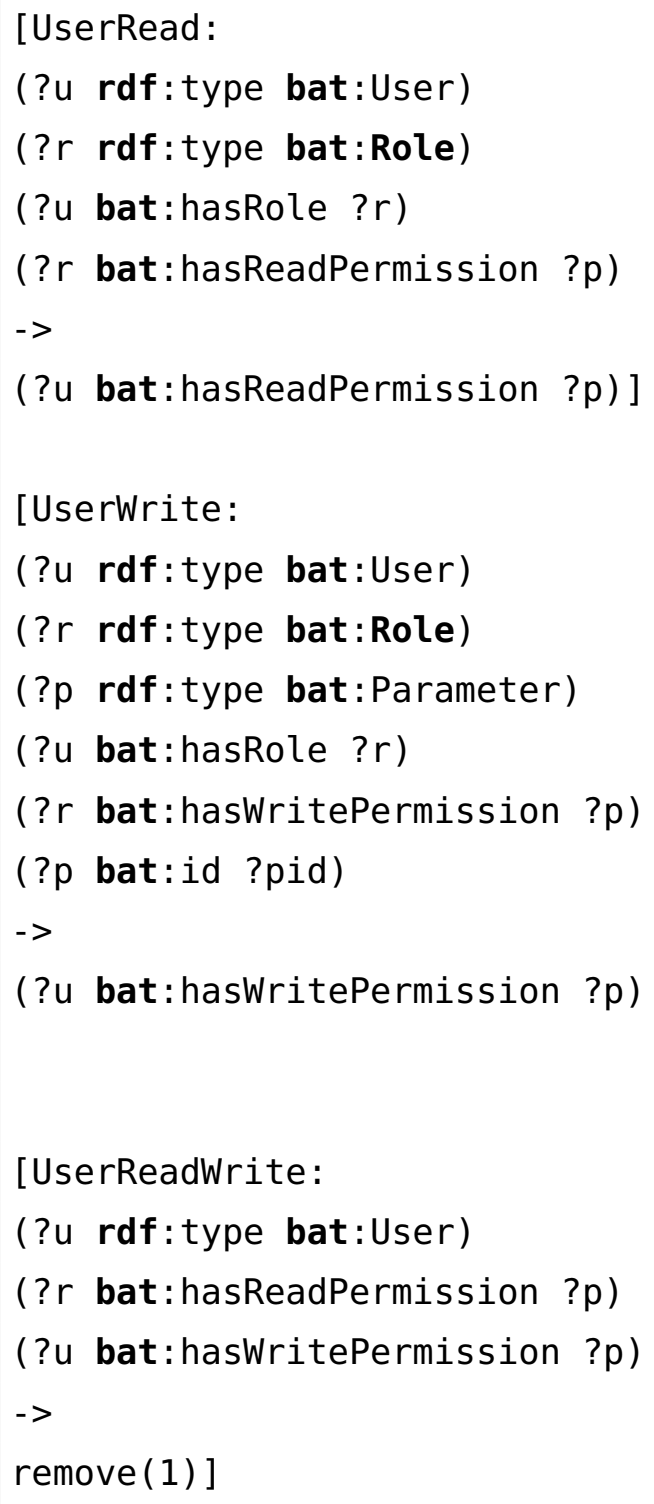

Listing 4.9 Formalization of rules in Jena

\section{Rule for User-Application permissions}

In the case that there are application installed for a client, the administrator of the client can decide the applications that each role is allowed access. The datatype property bat:appId indicates that this role is allowed to access the application with this id. As a matter of fact, a user should have access to all the applications that his roles have access. The rule shown in Listing 4.10 indicates that if a user have the a role and the role is allowed to access an application, then this user is allowed to access the application. 


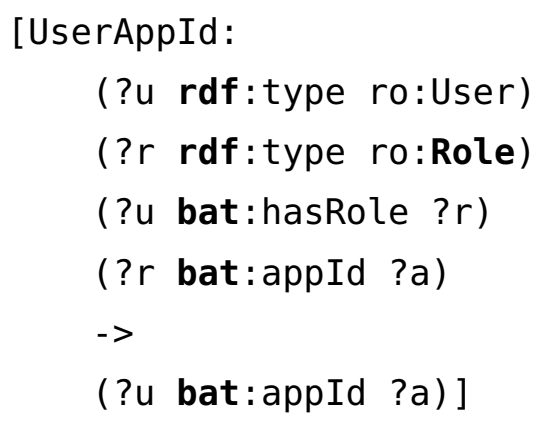

Listing 4.10 Formalization of rule for User-Application permissions in Jena

\subsubsection{Materialized Ontology Views for System KG}

According to the analysis of the read and write frequency, it is concluded that reading access is predominant in System KG. It has been noticed that the process of inference leads to high system resource usage. Accordingly, to improve the efficiency of system operation, the frequency of performing inference should be optimized. To achieve this objective, materialization is applied, which refers to the process to generate a Materialized Ontology View. As defined in [116], a Materialized Ontology View of a base ontology is "a (valid) ontology that consists solely of projections, copies, compressions, and/or combinations of elements of the base ontology, presenting a varying and/or restricting perception of the base ontology, without introducing new semantic data". Materialization allows to speed up query processing by explicating the implicit entailments which are sanctioned by the semantics of an ontology. [117] In other words, the outcome of inference should be stored as ready-to-consult facts in System KG for all the subsequent queries. And the inference should be only triggered when there are changes that can affect the result of the previous inference. This avoids the re-computation of derived information for every query.

Considering the circumstances of the platform that any change in the configuration of users, roles, devices and spaces may affect the result of the inference as they are the classes that have been involved in the rules. The change status of the named graph of a client is marked with a flag "changed". If changes are detected in the configuration of space, devices, parameter, users and roles of a client, the flag will be set as true. As each client has only one administrator who can modify the configuration, the inference will be triggered in two cases if flag "changed" is true. In the case that a query is made or the administrator has issued a logout request. By doing this, the system stability and query performance is optimised. 


\section{Chapter 5}

\section{Fine-grained access control for the Web of Things}

Considering the inherent characteristics of Web and the Internet of Things, how to ensure the security and privacy of both data and devices is one of the key issues to be concerned. Providing access to Things at the level of web and application brings new challenges for the IoT solutions. As a result, security is also an important issue to consider for the Web of Things. This chapter will explain about the authentication and authorization mechanism for the IoT platform, which has been developed on the basis of Web API and PKGs.

\subsection{Authentication and Authorization}

In the platform, the access control process is separated into two issues: authentication, i.e. "who you are" and authorization, which answers the question "what you can do".

Authentication ensures that the agent which is accessing the system is the right one. The agent could be either a person or software. In the platform, the authentication is realized by using both token-based method and ACL (Access Control List).

- A person (user) needs to authenticate himself by providing the proper username and password at the moment of login. If the login succeeds, a token will be returned. This token should be store in the local storage of the user and be put in the Authorization header of all the subsequent requests for accessing and manipulating the authorized data in the platform and control the authorized devices through the platform.

- A Border Router needs to provide its identifier in each request. Central Server keeps a list of valid identifiers and will only allow the request to pass if the appended identifier is in the list. 
Authorization is the process of specifying the access rights of agents to resources. In consideration of the complex situation in the platform, a fine-grained authorization mechanism is designed by combining the three most popular strategies for the definition of authorization policies:

- Discretionary Access Control (DAC), which restricts the access to objects based on the identity of subjects and/or groups to which they belong. And a subject with certain access permissions can transfer that permission on to any other subject [118][119][120]. In the case of the developed platform, for each organization/family that has registered for the platform, one administrator is defined, who has the rights to manage all the belonging users, infrastructures, facilities and assign the access permissions to different users.

- Role-Based Access Control (RBAC), which associates the permissions with roles and the users and can have various roles.[121][122] This strategy fits well with the requirement of multi-user system, providing a flexible way to manage the relationship between large amount of users and facilities. In the case of the developed platform, each user has a list of roles and it gets all the permissions that these roles hold.

- Attribute-Based Access Control (ABAC). The access rights are granted according to the attributes of users, resources, objects and environments. [123][124][83] Compared with the two strategies mentioned previously, ABAC is much more flexible and adaptive to the real-world situation, which enlarges the focus from users to the facilities and the surroundings. In the case of the developed platform, the access to devices are granted to roles according to the functionalities and locations of each device.

\subsection{Design of a Web API}

A Web API is designed for provide the communication with the developed IoT platform. The request can either comes from a user (administrator or non-administrator) or a Border Router. The administrator refers to the first user who is registered for a client, who has the rights to manage the configurations about users, devices, spaces, profiles and applications. Each client should provide a valid serial number of Border Router. The new added users of the same client are non-administrator, which is only allowed to access the authorized resources. To maintain a stable system, the URLs in the platform should be relative statistic, which ensures that individual web resources remain consistently at the same URL.[125]. To achieve this objective, a template is designed for the constructing of requests. The Web API is created by 
using JAX-RS, which is a Java API for creating RESTful Web Services [126]. The requests are conducted to the corresponding service in the Central Server.

As a multi-client, multi-user system, it is essential to have an effective access control system to ensure the security and privacy. Therefore, a module Security Manager is developed for the control of authentication and authorization in the IoT platform. The Security Manager is responsible to analyse each request and decide the appropriate reactions according to its content. The reactions could be to block/deny the request, to answer the query, or to send the device query or control command to the corresponding Border Router. For each agent, either a user or a Border Router, the Security Manager will generate a corresponding PKG to assure that the agent only access its authorized information.

In the following part, we will give a detailed introduction about request definition and the working process in Security Manager.

\subsubsection{Request Definition}

The Web API makes use of HTTP standards. Each request comprises four elements: Base URL, method, media type and payload.

- Base URL is a URL to identify the resource, which contains the information about objects and actions.

- Method is a verb which indicates the action of a request. Four verbs POST, GET, PUT and DELETE are used, which correspond to operations create, read, update and delete. In the platform, POST is used in the requests for user login as well as the ones for adding new objects. GET is used in all the requests for making queries about the objects. PUT is used in the requests for modifying and updating information of the objects. And DELETE is used in the requests for user logout and the deletion of objects.

- Media type indicates the Internet media type for the exchanged data. In this platform, JSON is selected as the main media types.

- Payload carries the data that is needed to perform a request. The syntax and format of the content should be in accordance with the media type selected.This element is only supported in the request with methods POST and PUT. 


\subsubsection{Definition of the representation of resources}

A representation is a machine-readable description of the state of a resource. The resources in the platform are represented in the form of JSON. Two examples of the definition of resource representation in the IoT platform are given in this part.

In the platform, for all the POST, PUT and DELETE request, a resource message is returned in the form of JSON object to show the result of the operation. Listing5.1 shows the JSON representation of the resource "message", which is contained in the response of a login request. 


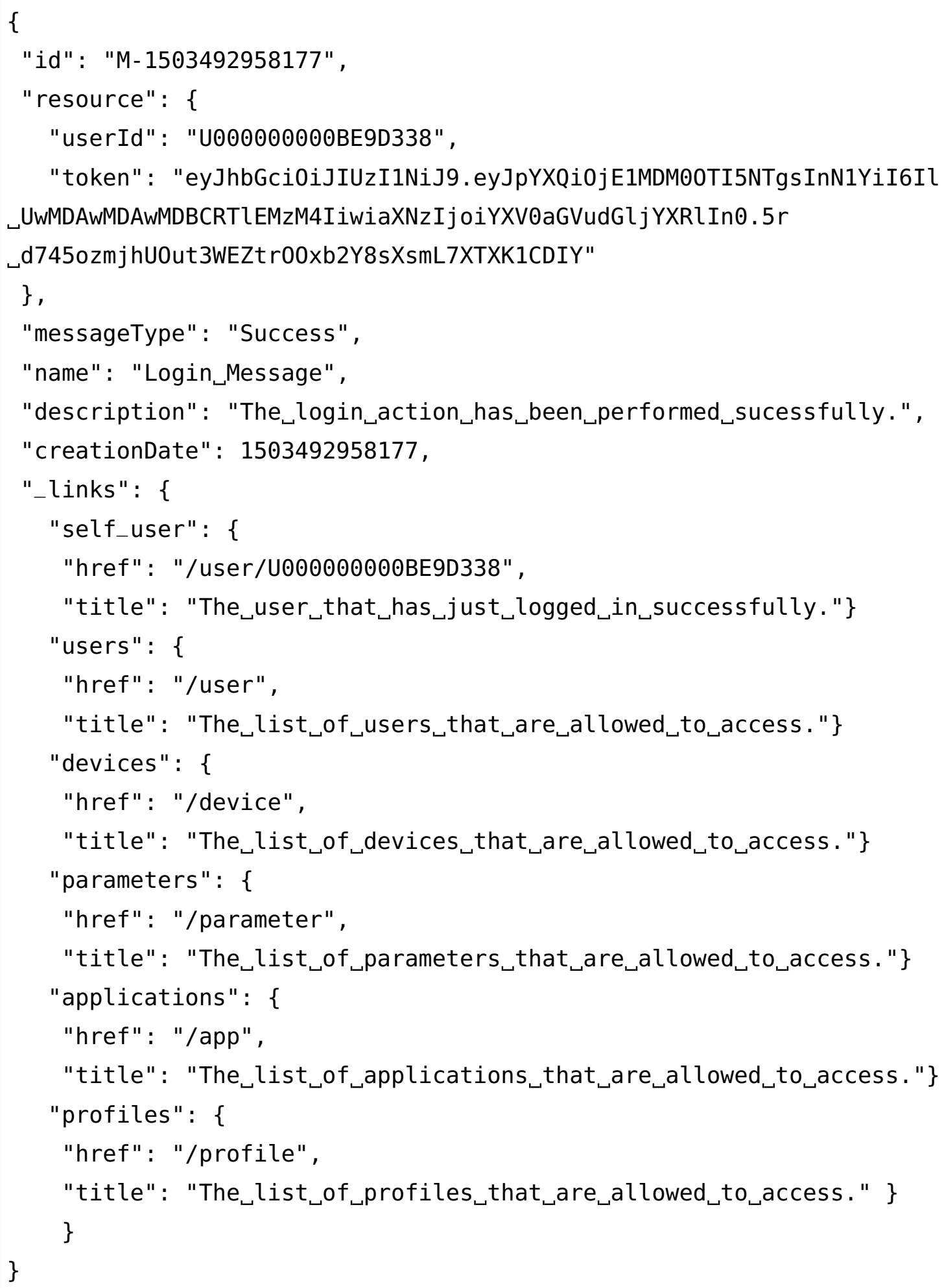

Listing 5.1 JSON representation of message resource

The representation of the resource message includes the following fields: 
- "id", which refers to the identifier of the message.

- "resource", which refers to a list of key/value pairs that can be used for the further request. In the case that the message is a response for the login action, a valid token is indicated in this field, which should be retrieved and included in the Authorization header of the subsequent requests.

- "messageType" refers to the type of this message, which can be Success, Error or Info.

- "name" refers to the name of the message.

- "description" is the human-readable text for showing to the users.

- "creationDate" indicates the time stamp of this message.

- "_links" indicates a set of links that can be followed for the subsequent operations, where the key such as "users" and "devices" refers to the relationship with the resource, the field "href" indicates the target URI and the "title" is a human-readable text to explain this link resource.

For a GET request, the result of query is returned in the form of a JSON object or a list of JSON objects. Listing 5.2 shows the JSON representation of a parameter resource, which is one of the main concepts used in the IoT platform. 


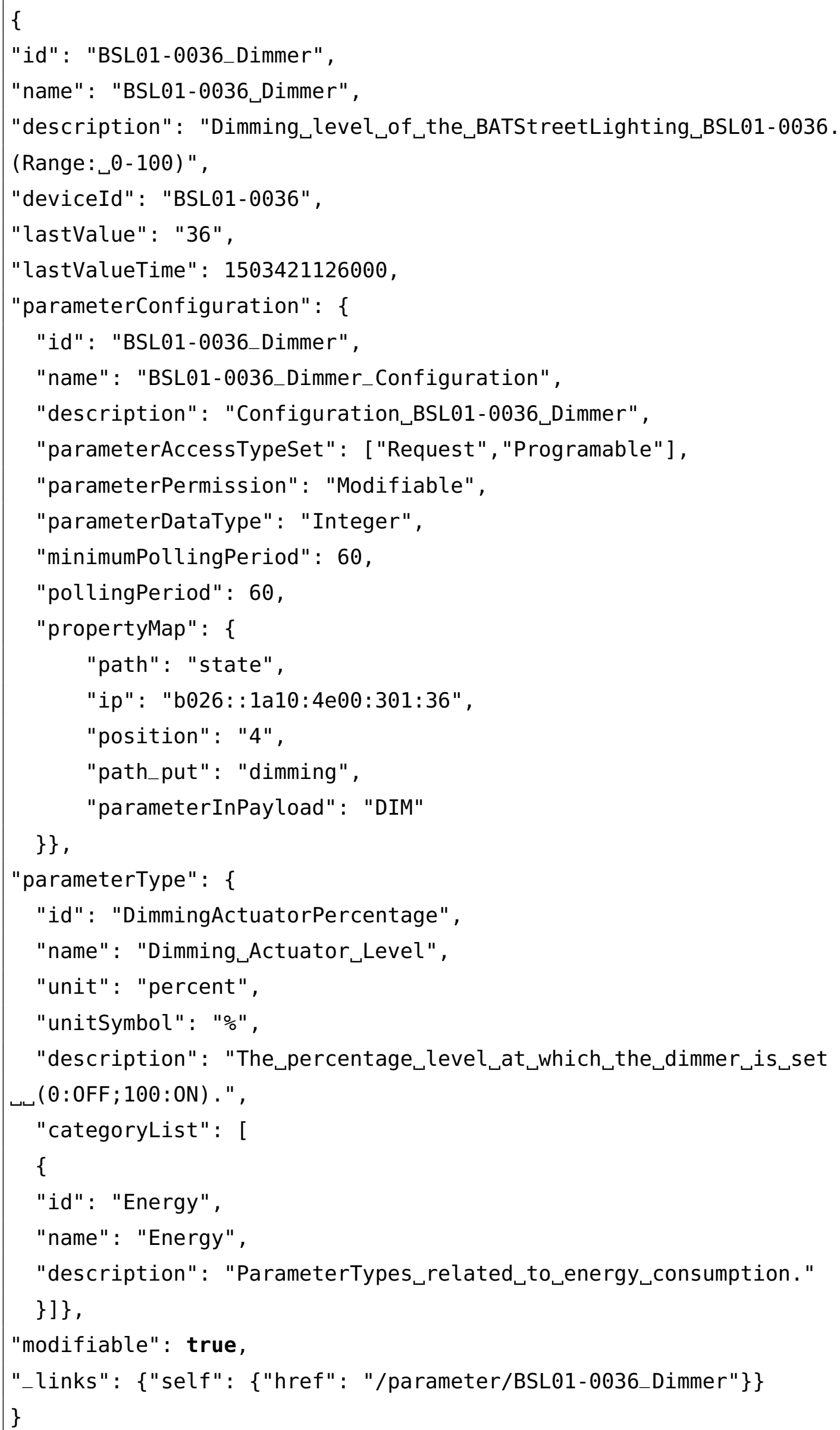


The representation of the resource parameter includes the following fields:

- "id" refers to the unique identifier of the parameter.

- "name" indicates the name of the parameter, which is a human-readable text for the information of users.

- "description" is a human-readable sentence, which gives the detailed information about this parameter.

- "deviceId" indicates the identifier of the device to which this parameter belongs.

- "lastValue" and "lastValueTime" indicates the latest state of the parameter and the corresponding time-stamp

- "parameterConfiguration" is an embedded object of parameter, which contains the information about the configuration including the "parameterAccessTypeSet", "parameterPermission", "parameterDataType", "mininumPollingPeriod" and "pollingPeriod".

- "propertyMap" is an embedded object of parameterConfiguration, which contains a list of properties that are necessary for the driver to query or modify the value of this parameter by communicating with the device.

- "parameterType" is an embedded object of the parameter, which indicates the type of the parameter, which include the information of presentation, including "unit", "unitSymbol" and "description". The categoryList is an embedded object of the parameterType which declares the information about the category of the parameterType.

- "modifiable" indicates that if the parameter can be modified.

- "_links" indicates the links associated with the resource. The "self" link shown here denotes the link for the resource itself.

\subsubsection{Templates for request construction}

Table 5.1 shows the templates for constructing requests for the interaction with the platform. The constructed requests are provided to the user and web-applications to communicate with the platform. The set of ResourceClass in the table includes user, space, device, artifact, parameter, role, profile and app. 


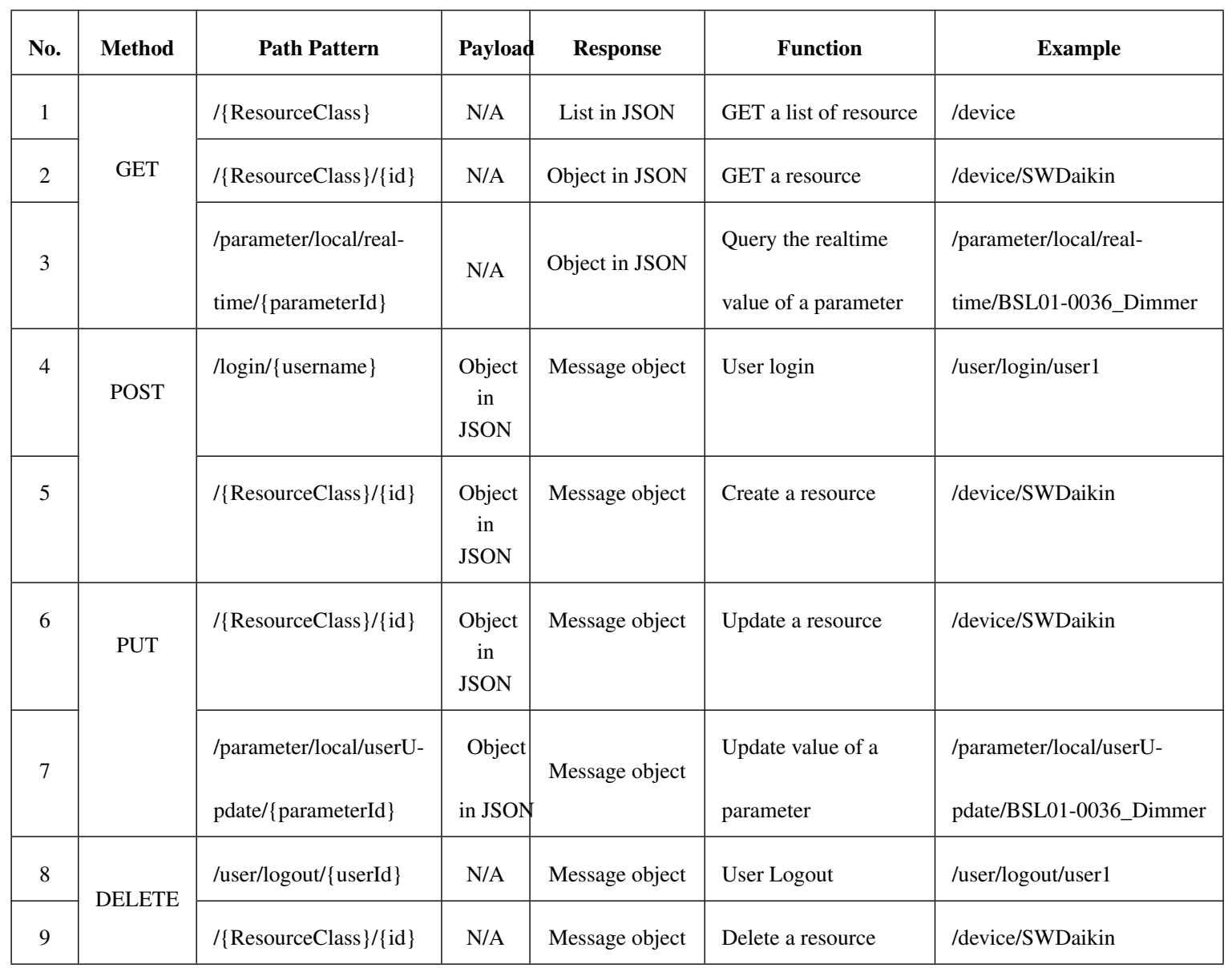

Table 5.1 Templates for constructing requests

An explanation of each entry is given in the following:

- The entries No.1 and No.2 are GET requests for retrieving information about resources of a specific class. Entry No. 1 shows that if only the ResourceClass is provided, all the resources of this class will be returned. If the ID is provided, as shown in entry No. 2 , the resource with this ID will be returned.

- The entry No.3 is a GET request for querying the realtime value of the specific parameter. When the request is received, the Central Server will communicate with the corresponding Border Router of this parameter to ask for its value at that moment. The returned response contains the parameterId, value and the time-stamp. This value will not be stored in the Data Warehouse.

- The entry No.4 is a request for user login. The password is sent in the payload as JSON object in the form of $\{$ password": $\{$ pwd $\} "\}$, where pwd is the input password. If it 
succeeds, the server will response with a message, containing the token generated on the basis of userId and time-stamp. Meanwhile, in the Security Manager, a PKG is created corresponding to the token.

- The entry No.5 is to create a resource of a specific class, the ID is indicated in the URL and the representation of the resource is provided in the payload. If a resource with the ID already exists, the request will be rejected.

- The entry No.6 is to update the information of a specific resource with the ID provided in the URL. If a resource with the provided ID already exists, its information will be replaced with the data provided in the payload. Otherwise, the request will be rejected with the error message "The object to be updated does not exist".

- The entry No.7 is to change the value of a specific parameter so as to control the device. The ID of the parameter should be indicated in the URL and the new value should be included in the payload in the form of JSON object $\{$ "setValue": $\{$ newValue $\}$ " where newValue should be replaced by the desired value. The new value will be sent to the corresponding Border Router of this parameter to execute in the local network.

- The entry No.8 is a logout request which will result in the deletion of all the entries related with this user, such as Token-PKG and Token-ClientID.

- The entry No.9 is to delete the specific resource of a class with the ID given in the URL. If the resource with the ID exists, it will be deleted and a success message will be returned. Otherwise, an info message will be returned, indicating that "The object to be deleted does not exist".

\subsection{Security Manager Composition}

By defining a proper Web API, the agents are able to get information of users, devices or control a device easily through a request. While with the involvement of physical devices and multi-user scenario, an effective access control mechanism is imperative for authentication and authorization. As a result, a module Security Manager has been implemented for performing this task. As illustrated in Fig. 5.1, the Security Manager consists of an Authorized PKG Manager, a KG handler and an Authorization Validator. They work together with Request Analyser to perform the authentication and authorization control of the system. As a result, from the point of view of security functionality, the Request Analyser is also regarded as part of the Security Manager. A detailed introduction of each module is given in the following part: 
- Request Analyser is responsible for analysing the request and communicating with all the other three components to decide the subsequent actions.

- Authorization PKG Manager is responsible for the storage of all the PKGs for different agents, which can be a Border Router, an administrator or a non-administrator user. Each PKG is stored corresponding to a token or the ID of a Border Router.

- KG Handler is responsible for managing all the named graphs in System KG and generating PKGs for agents. Two modules are included: the Named Graphs Manager for the generation of named graph URLs on the basis of the ID of a client or a Border Router, and the PKG Generator for the generation of PKGs, which will be explained in detail in Section 5.5.1. It also tracks the change status of each client's named graph to decide if the inference should be triggered.

- Authorization Validator is to control if a parameter can be modified by a nonadministrator user and if a token has expired. For each token of a non-administrator user, it holds a list of all the writable parameters IDs (WPL). A request can only change the value of a parameter if the ID of the parameter is in the WPL corresponding to its token. 


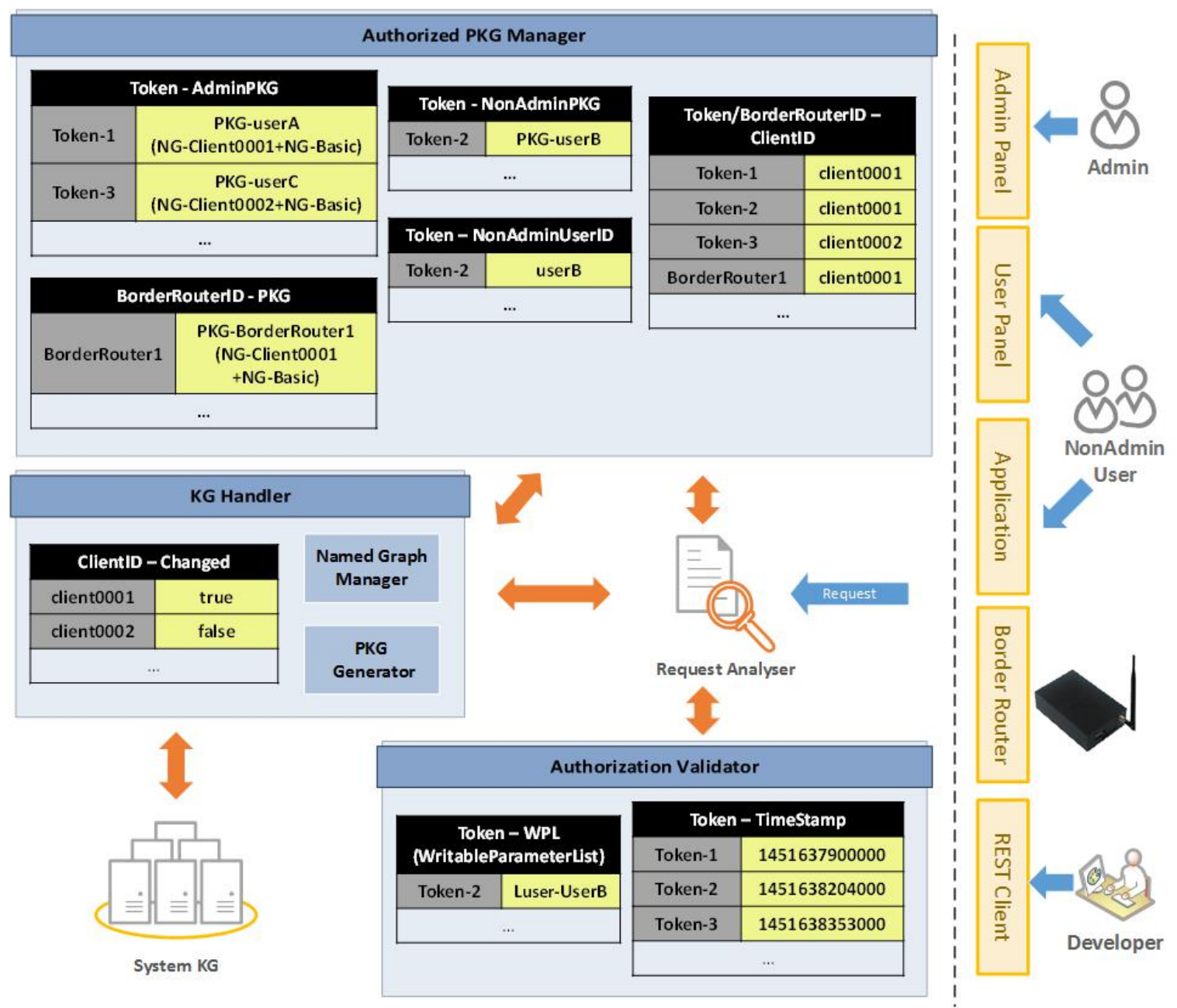

Fig. 5.1 Composition of the Security Manager

\subsection{Request Analysis}

As long as a request is received, the Request Analyser checks each elements of the request, including the method and the URL and then communicates with all the modules in Security Manager to decide the subsequent actions. Fig. 5.2 illustrates the workflow of request analysis in Security Manager. Each step is marked with different colours which indicate the main module involved in the action. 


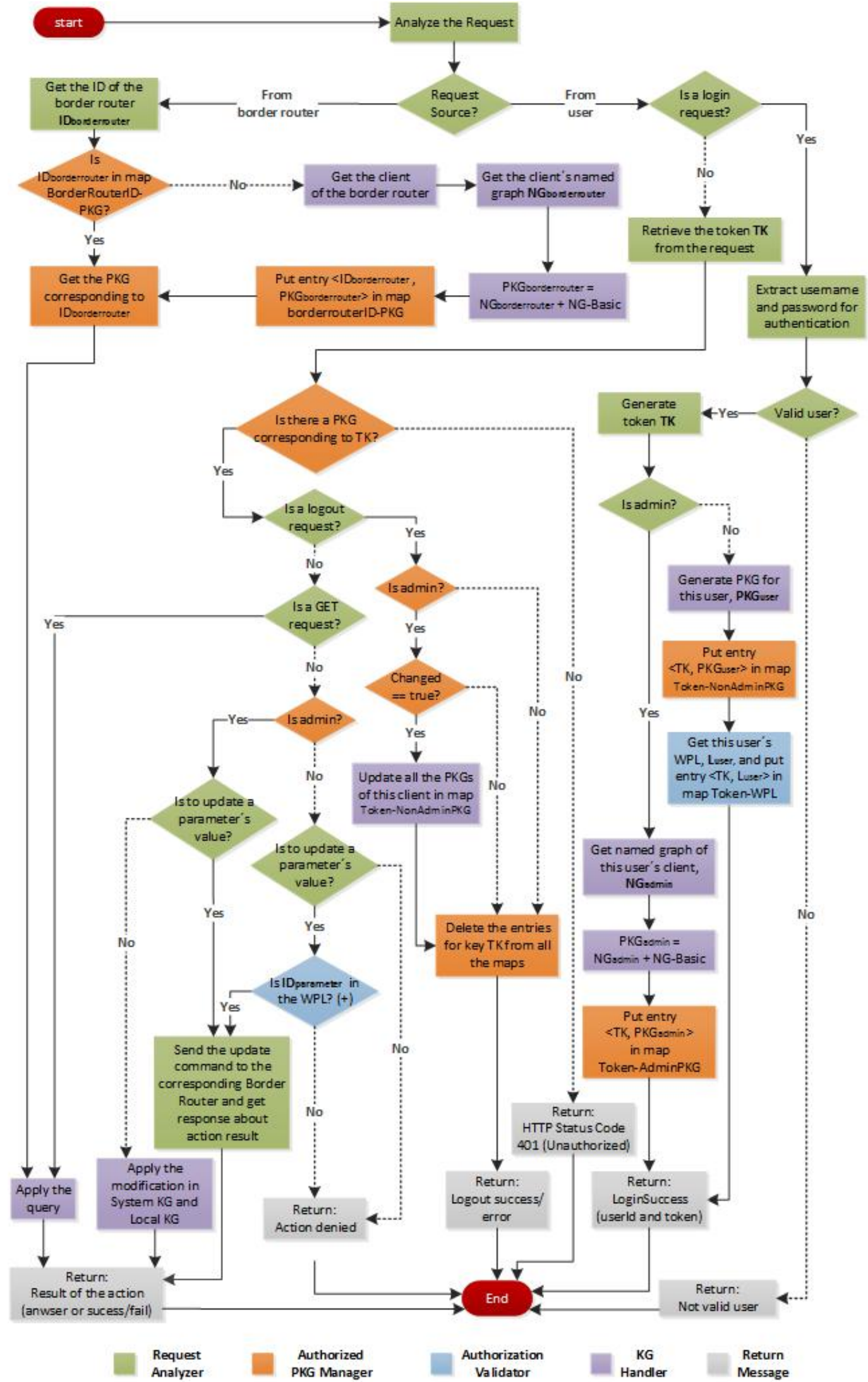

Fig. 5.2 Workflow of request analysis 
Basically, the requests can be classified into two categories according to the agents that issue them, either from a Border Router or a user.

\subsubsection{Request from Border Router}

A Border Router issues requests to the Central Server for sending the information that has been collected in the local network. The requests are issued in two cases:

- When a new device has been installed in the local network, the information of the device should be sent to the Central Server.

- Periodically, each Border Router sends the measurements collected in its local network to the Central Server. The period between each request is configurable.

Fig. 5.3 shows the workflow of request analysis for the requests from Border Routers.

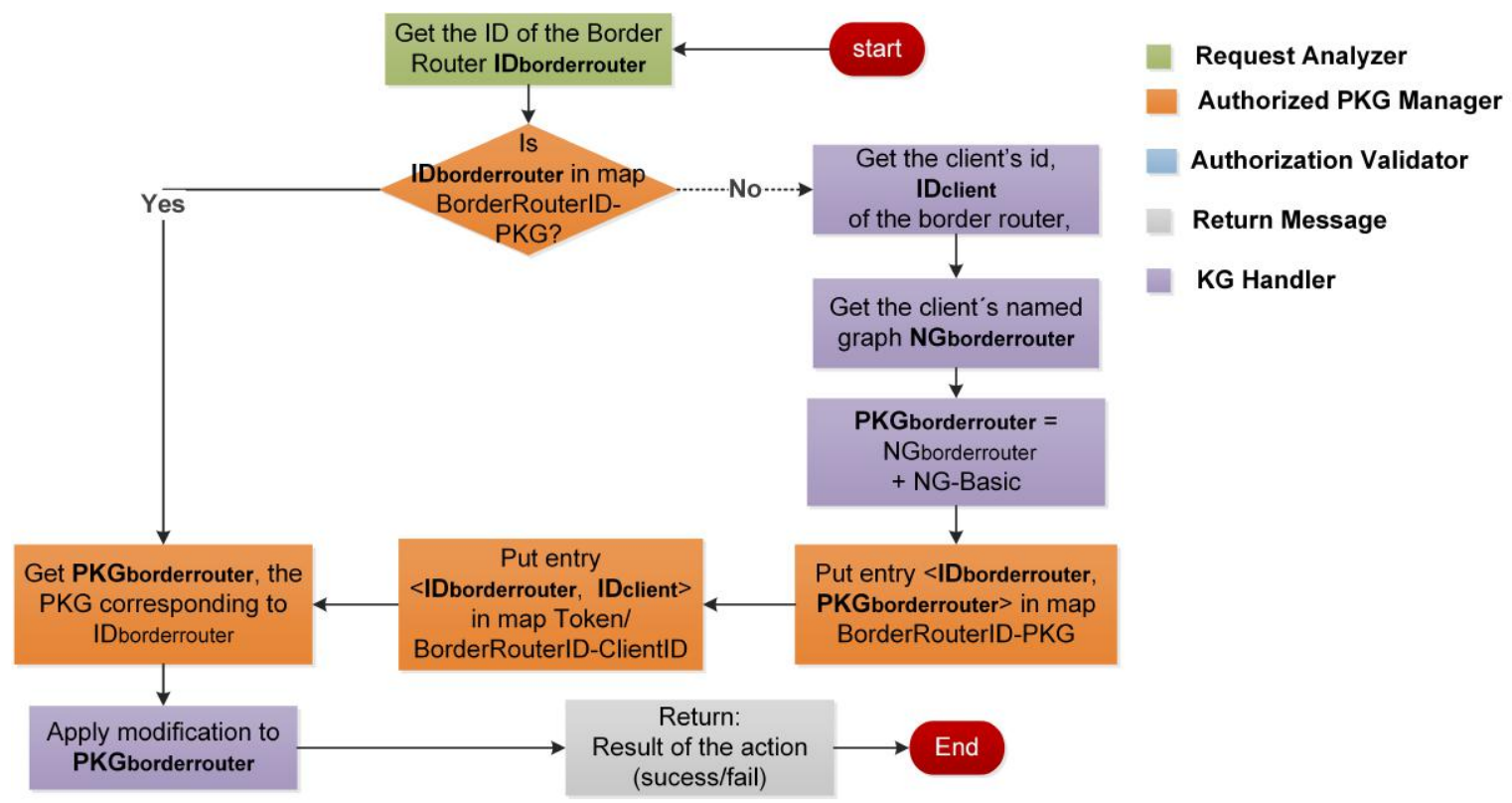

Fig. 5.3 Workflow of request analysis for the requests from Border Routers

All the requests that sent by a Border Router contains the keyword "/borderrouter" in their URL. If the keyword is detected in the URL, the subsequent steps will be taken by the Security Manager:

[1] Get the ID of the Border Router. After splitting the URL based on "/", the last element in the returned array indicates the serial number of the Border Router, denoted by $\mathrm{ID}_{\text {borderrouter }}$. 
[2] If there is a PKG corresponding to ID $_{\text {borderrouter }}$ in the map BorderRouterID-PKG, then this PKG is used to complete the actions indicated in the request.

[3] If $\mathrm{ID}_{\text {borderrouter }}$ is not in the map BorderRouterID-PKG, a PKG will be generated for it by the KG handler. Each Border Router belongs to a client. Therefore, The PKG for a Border Router is the same as the PKG for its client. The generation of PKG is fulfilled in four steps:

(a) Get the corresponding client of $\mathrm{ID}_{\text {borderrouter }}$ by consulting the System KG, denoted as $\mathrm{ID}_{\text {client }}$.

(b) Get the named graph of this client according to $\mathrm{ID}_{\text {client }}$, denoted as $\mathrm{NG}_{\text {borderrouter }}$

(c) Obtain the PKG for the Border Router by combining NG-Basic and $\mathrm{NG}_{\text {borderrouter }}$, denoted as $\mathrm{PKG}_{\text {borderrouter }}$.

(d) Put the new entry $<\mathrm{ID}_{\text {borderrouter }}, \mathrm{PKG}_{\text {borderrouter }}>$ in map BorderRouterID-PKG

(e) Put the new entry $<\mathrm{ID}_{\text {borderrouter }}$, $\mathrm{ID}_{\text {client }}>$ in map Token/BorderRouterID-ClientID.

[4] Get the PKG corresponding to the $\mathrm{ID}_{\text {borderrouter }}, \mathrm{PKG}_{\text {borderrouter }}$ from map BorderRouterIDPKG.

[5] Apply the modification action indicated in the request to $\mathrm{PKG}_{\text {borderrouter }}$.

[6] Return the result of the action to the Border Router, which is the success/fail information about the modification.

\subsubsection{Request from user}

When a user request is received, the Request Analyser first checks if it is a login request, which will result in the generation of PKGs. Fig. 5.4 shows the workflow of the request analysis for a login request and the generation of PKGs.

\section{Login Request}

A login request is sent with the verb POST and with the keyword "/login" in the URL. When such a request is received, the Request Analyser extracts the username from the URL and the password from the payload and sends them to the System Data Warehouse for user authentication. If the authentication information provided is not correct, an error message "Not valid user" will be returned. Otherwise, a token TK is generated on the basis of username and timestamp and a PKG is generated correspondingly. The process to generate PKG is 
different for administrator and non-administrator user. Fig. 5.4 shows the workflow of request analysis for a login request.

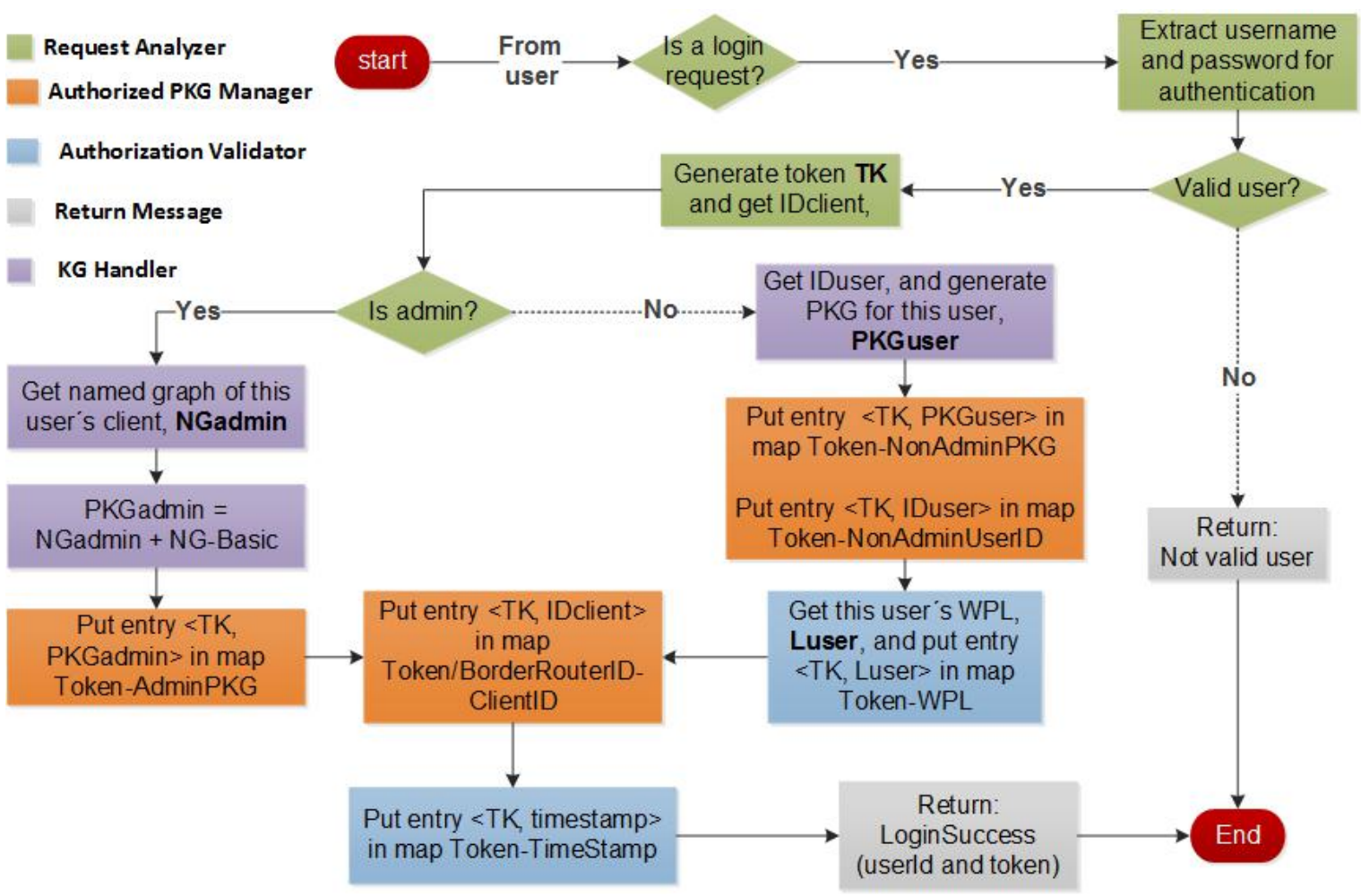

Fig. 5.4 Workflow of request analysis for a login request

For administrator If the user is an administrator, a PKG should be generated and stored by KG Handler in the following steps:

[1] Checks with the System KG and get the clientID of the user, denoted as ID $_{\text {client }}$.

[2] Get the named graph for the client according to the clientID, denoted as $\mathrm{NG}_{a d m i n}$

[3] Combine NG-Basic and $\mathrm{NG}_{a d \min }$ as $\mathrm{PKG}_{a d m i n}$

[4] Put the entry $<\mathrm{TK}, \mathrm{PKG}_{a d m i n}>$ in map Token-AdminPKG.

For non-administrator If a user is not administrator, the following steps should be performed:

[1] Get the id of the user, denoted as $\operatorname{ID}_{\text {user }}$ and the ClientID of the user, $\operatorname{ID}_{\text {client }}$ 
[2] The KG Handler needs to retrieve from System KG all the data that the user can access and put them in a knowledge graph according to the $\operatorname{ID}_{\text {user }}$ and $\mathrm{ID}_{\text {client }}$, denoted by $\mathrm{PKG}_{\text {user }}$.

[3] Put the new entry $<\mathrm{TK}, \mathrm{PKG}_{\text {user }}>$ in map Token-NonAdminPKG, and add the entry $<\mathrm{TK}, \mathrm{ID}_{\text {user }}>$ to the map Token-NonAdminUserID.

[4] The Request Analyser retrieves from $\mathrm{PKG}_{\text {user }}$ the IDs of all the parameters that this user can modify and put them in a Writable Parameter List, $\mathrm{L}_{\text {user }}$. The generated new entry $<\mathrm{TK}, \mathrm{L}_{\text {user }}>$ is stored in map Token-WPL in Authorization Validator.

In both of the cases, two more steps are performed after accomplishing the steps introduced above, :

- Put the entry $<\mathrm{TK}, \mathrm{ID}_{\text {client }}>$ in map Token/BorderRouterID-ClientID.

- Get the current time as timestamp and put the entry $<\mathrm{TK}$, timestamp $>$ in map TokenTimeStamp.

\section{Logout Request}

In the case that a request is not to perform a login action, the following steps are illustrated in Fig. 5.5:

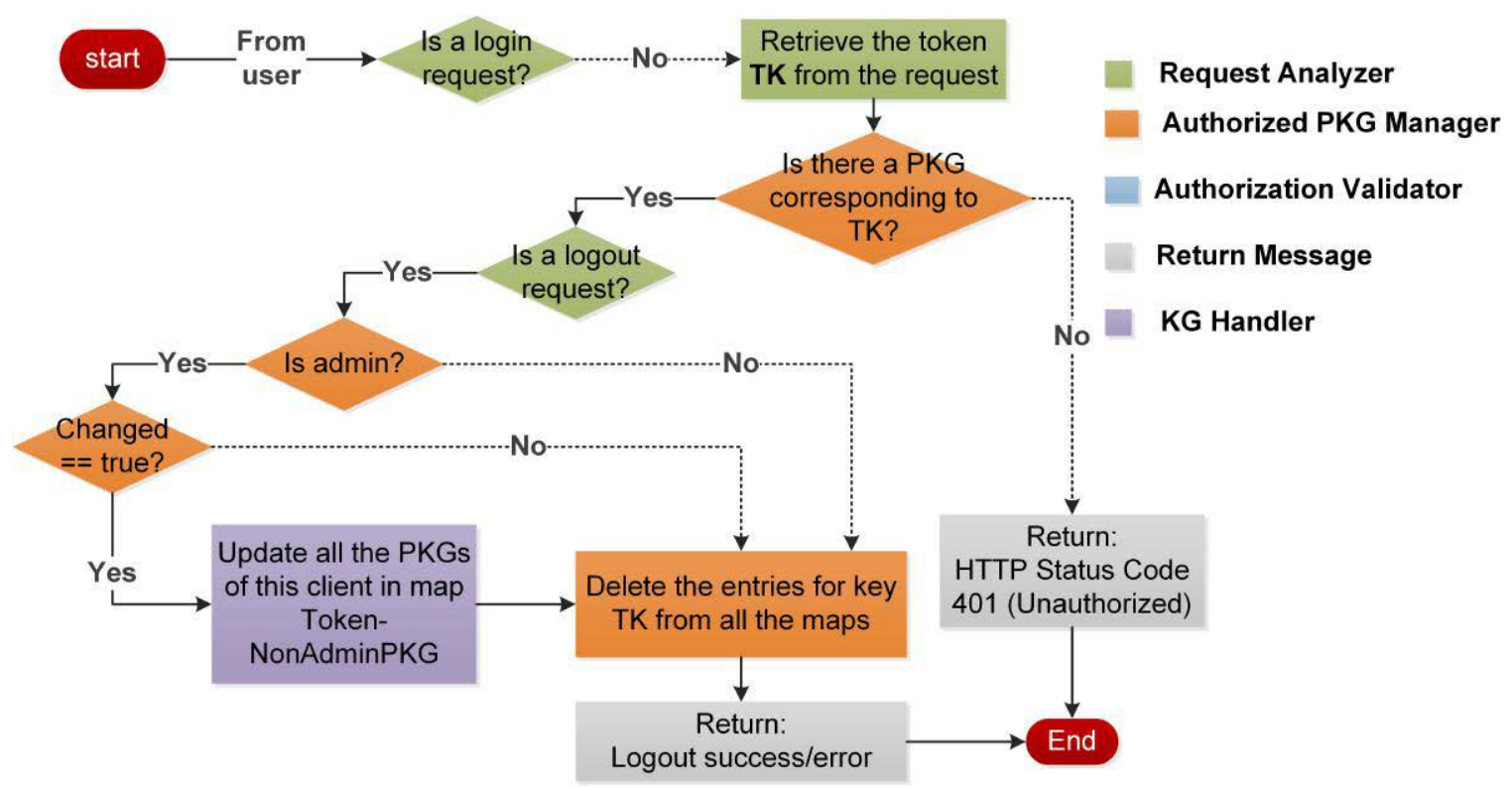

Fig. 5.5 Workflow of request analysis for the Logout request 
[1] If the request is not to perform a login action, Request Analyser will check the Authorization header to retrieve the token TK.

[2] If in the Authorized PKG Manager, there is no corresponding PKG for TK, either in map Token-AdminPKG or Token-NonAdminPKG, the request should be denied and a response with HTTP status code 401 (Unauthorized) is returned.

[3] Otherwise, Request Analyser will check if the request is a DELETE request that contains the keyword "/logout" in URL, which performs a logout action.

When a user logs out from the system, all the entries for the key TK should be removed from the Security Manager, which distribute in the following maps:

- Token-AdminPKG

- Token-NonAdminPKG

- Token-NonAdminUserID

- Token/BorderRouterID-ClientID

- Token-WPL

- Token-TimeStamp

Moreover, if the user is an administrator and the corresponding PKG has been changed, it is necessary to regenerate PKGs for all the non-administrator users from the same client and update the map Token-NonAdminPKG with the new PKGs.

\section{Request for query or modification}

In the case that a request is neither for login nor for logout, the subsequent action varies according to three conditions:

- The method of request, which could be GET, PUT, POST and DELETE.

- If the user is an administrator, i.e. if TK is contained in the map Token-AdminPKG.

- If the request is to update the value of a parameter.

Fig. 5.6 illustrates the different cases and the corresponding actions performed in the Security Manager. 


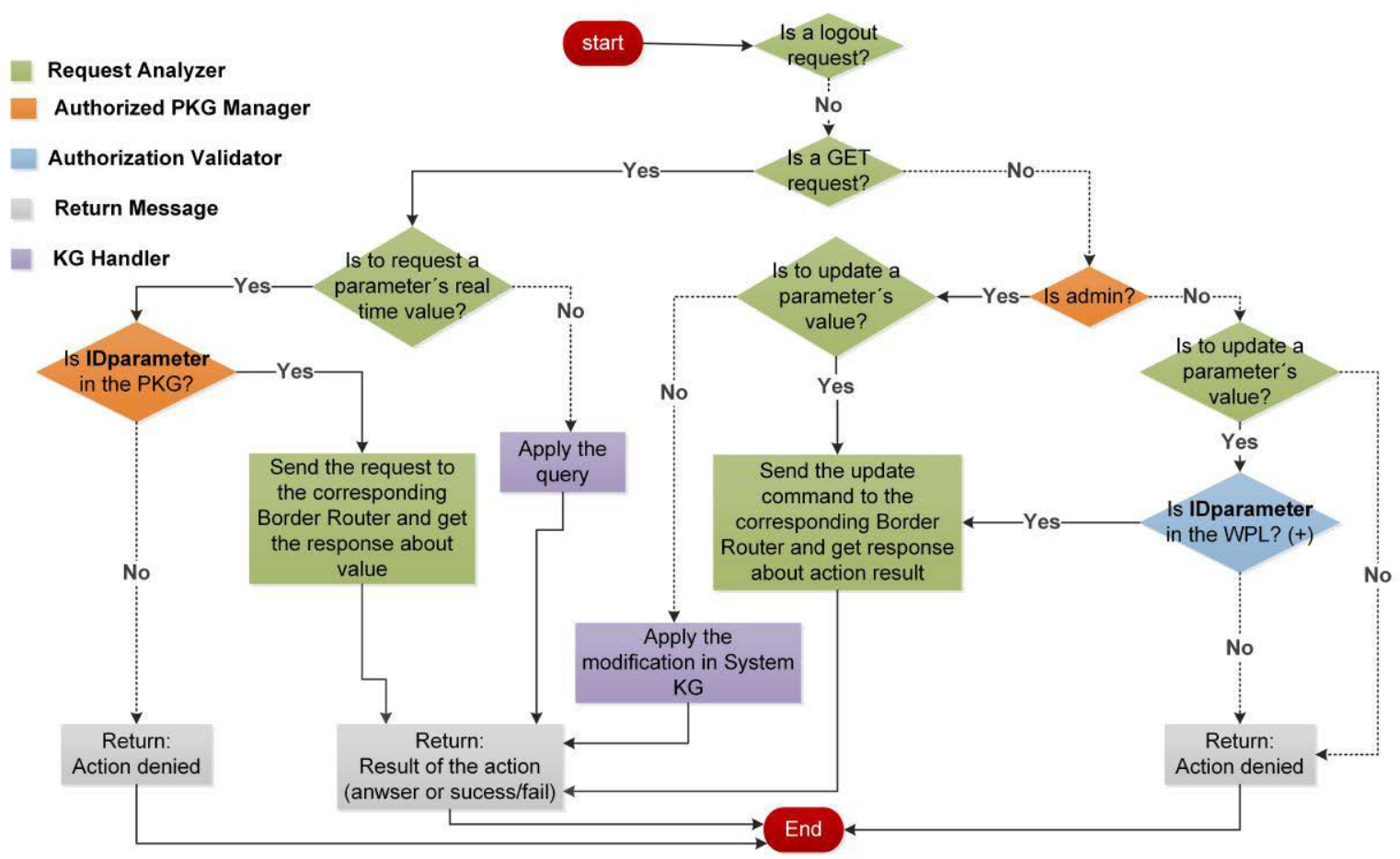

Fig. 5.6 Workflow of request analysis for the query or modification request

[1] If the request is a GET one, then it is allowed to carry out against the user's corresponding $\mathrm{PKG}$.

[2] In the case that the request is to ask for the realtime value of a parameter, the Security Manager will first check that if the ID of the parameter is in the corresponding PKG of this user. If yes, the request will be sent to the corresponding Border Route, which will then communicate with the device for its realtime value. Otherwise, the request will be denied.

[3] If the request is a POST, PUT or DELETE and the user is an administrator, the request is permitted and the corresponding modification is made.

The GET request is used to make query about the user's data such as "show all the devices the user can access", which is always permitted for querying its own PKG. The POST, PUT and DELETE requests are used to modify informations in the System KG such as "add new users", "delete a role" or "modify the data about a space". As mentioned previously, only the administrator can modify the data for the client. Therefore, the POST, PUT and DELETE requests are permitted if the user is an administrator and will be denied otherwise.

Apart from the cases mentioned above, there is an exception if the request is to control a device by updating the value of a writable parameter that belongs to the device: 
- If the user is an administrator, the update command will be sent to the corresponding Border Router to control the device in its local network .

- For non-administrator users, the Request Analyser needs to first check with Authorization Validator that if $\mathrm{ID}_{\text {parameter }}$ is included in the Writable Parameter List (WPL) that corresponds to this TK. If ID $\mathrm{IDaramet}_{\text {is }}$ in the list, the command will be sent to the corresponding Border Router. Otherwise, the request will be denied.

The operation is carried out by a PUT request with the URL

"/parameter/local/userUpdate/ $\left\{\mathrm{ID}_{\text {parameter }}\right\}$ ", where $\mathrm{ID}_{\text {parameter }}$ indicates the ID of the target parameter, the payload of the request should be written in the form of JSON object \{"setValue":" \{newValue\}"\} where the newValue indicates the value to which the parameter should be set to.

\subsubsection{Security consideration}

In the platform, all the requests are encrypted with a valid certificate and sent over SSL to ensure the security. And the access control mechanism in the platform is designed to prevent harmful requests. The requests not only refer to the ones that come from a web browser, but also those that are issued by software agents. To test the reliability of the developed access control system, two cases have been considered where there might be threats of security:

- If a REST client is used to simulate a user and send a request to Central Server, the request will not be accepted unless a valid token is included in the authorization header of the request. The valid token is a JSON Web Token generated by Security Manager on the basis of username and timestamp, which can only be achieved by sending a login request with a valid username and password. Meanwhile, the request with a valid token can only affect the PKG for this token. As a result, the designed mechanism prevents the fake requests from accessing the data in System KG.

- If a REST client is used to simulate a Border Router to send request to Central Server, it should contain a valid BorderRouterID. The BorderRouterID is its serial number, which is assigned by the manufacture. In Central Server, an Access Control List is kept, which contains all the valid BorderRouterIDs. The request will not be accepted unless the provided BorderRouterID is in the list and the Border Router has been registered with a client. As a result, a REST client can not imitate a Border Router to change the information in System KG unless it can "guess" correctly the BorderRouterID and the client who has registered the Border Router, which is a quite tough task. 


\subsection{PKG management}

As mentioned previously in Chapter 4, in order to achieve fine-grained access control, a new concept "Private Knowledge Graph" (PKG) has been proposed to restrict the scope of the resource that an agent can access. The generation of PKG is implemented by applying several Semantic Web technologies, including ontology engineering, inference and SPARQL. In Security Manager, the KG Handler is responsible for the generation of PKGs for all the agents. A PKG can be a combination of several named graphs or an amount of RDF statements, which only contains the information that the agent is authorized to access. Fig. 5.7 illustrates the relationships among all the tokens, users, clients and Border Routers involved in the example described in section 5.3. The following parts will explain in detail about the PKG generation, storage, expiration and update.

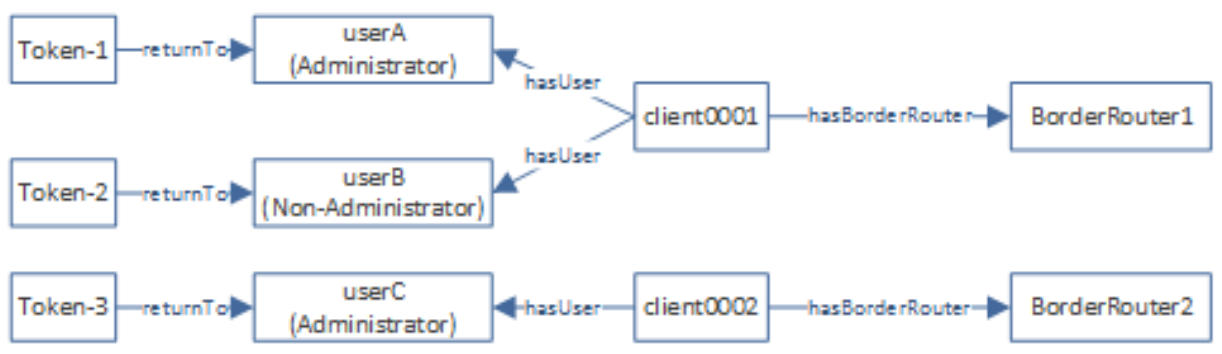

Fig. 5.7 Description of relationships among entities in the given example

\subsubsection{Generation and storage}

Depends on the agent that uses the PKG, the process to generate PKG are different. In total, there are three manners for the generation of PKGs: for Border Router, for administrator and for non-administrator. The PKGs are respectively stored in different maps in Security Manager according to the agents that they work for.

\section{PKG for Border Router}

The PKG for a Border Router contains all the information that belong to its client, which consists of three parts: named graph of the client, deductions named graph of the client and NG-Basic. In total, there are three steps of the PKG generation for a Border Router. Taking the BorderRouter1 in Fig. 5.7 as example, the PKG generation for a Border Router has four steps:

[1] The Named Graph Manager checks with System KG to obtain the clientID corresponding to this Border Router. The BorderRouter1 belongs to client0001. 
[2] The Named Graph Manager generates the URLs of demanded named graphs. In this case, two URLs are obtained:

- "batmp.cedint.upm.es/BATOnt/client0001"

- "batmp.cedint.upm.es/BATOnt/inf/client0001"

[3] The PKG Generator combines the following three named graphs together as PKGBorderRouter1 and put entry <BorderRouter1, PKG-BorderRouter1> in the map BorderRouterID-PKG:

- "batmp.cedint.upm.es/BATOnt/client0001"

- "batmp.cedint.upm.es/BATOnt/inf/client0001"

- NG-Basic

[4] Put a new entry <BorderRouter1, client0001> to the map Token/BorderRouterIDClientID.

\section{PKG for administrator}

As an administrator has the right to change all the information of human resource and infrastructures of his client, the PKG for an administrator contains all the information that belong to his client. The PKG consist of three named graphs: NG-Basic, named graph of the client and the deductions named graph of this client. In Fig. 5.7, userA is the administrator. The PKG generation for an administrator has six steps:

[1] The Named Graph Manager queries the System KG and obtains the corresponding clientID of the user, which is client0001 in this case.

[2] By knowing the clientID, the URLs of the two demanded named graphs are generated:

- "batmp.cedint.upm.es/BATOnt/client0001"

- "batmp.cedint.upm.es/BATOnt/inf/client0001"

[3] The PKG Generator combines the following three named graphs together as PKGuserA:

- "batmp.cedint.upm.es/BATOnt/client0001"

- "batmp.cedint.upm.es/BATOnt/inf/client0001"

- NG-Basic 
[4] Put a new entry <Token-1, PKG-userA $>$ in the map Token-AdminPKG.

[5] Put a new entry <Token-1, client0001> to the map Token/BorderRouterID-ClientID

[6] Put new entry <Token-1, 1451637900000> to the map Token-TimeStamp.

\section{PKG for non-administrator user}

For an non-administrator user, the PKG should be restricted to the informations that is related to the user in some way. In the platform, from the perspective of control, the relevance of an entity to a user is decided by its relevance to the parameters that the user has permission to access. As a result, the PKG is generated by searching all the entities that have relationship with the parameters, either direct or indirect. Meanwhile, for non-administrators, apart from the PKG, a Writable Parameter List (WPL) should also be retrieved to disclose all the parameters that the user is authorized to modify.

The PKG generation for a non-administrator has six steps:

[1] The Named Graph Manager queries the System KG and obtains the corresponding clientID of the user.

[2] By knowing the clientID, the URLs of demanded named graphs are generated, which are:

- "batmp.cedint.upm.es/BATOnt/client0001"

- "batmp.cedint.upm.es/BATOnt/inf/client0001"

[3] The PKG Generator combines the named graphs "batmp.cedint.upm.es/BATOnt/client0001", "batmp.cedint.upm.es/BATOnt/inf/client0001" and NG-Basic together as a new named graph, denoted as NG-client

[4] Apply the CONSTRUCT SPARQL query shown in Listing 5.3 against NG-client, where the text USER_ID refers to the ID of the user.

[5] Generate the WPL $\mathrm{L}_{\text {user }}$ by applying the SPARQL query in Listing 5.4, where the text USER_ID refers to the ID of the user. In this case, by replacing USER_ID with userB, the list $\mathrm{L}_{\text {user }}$-userB is generated for Token-2.

[6] After having obtained PKG-userB and list $\mathrm{L}_{\text {user }}$-userB, the entries are added to the corresponding maps:

- Entry <Token-2, PKG-userB > to map Token-NonAdminPKG 
- Entry <Token-2, userB > to map Token-NonAdminUserID

- Entry <Token-2, client0001> to map Token/BorderRouterID-ClientID

- Entry $<$ Token-2, $\mathrm{L}_{u s e r}$-userB $>$ to map Token-WPL

- Entry <Token-2, 1451638204000> to map Token-TimeStamp 


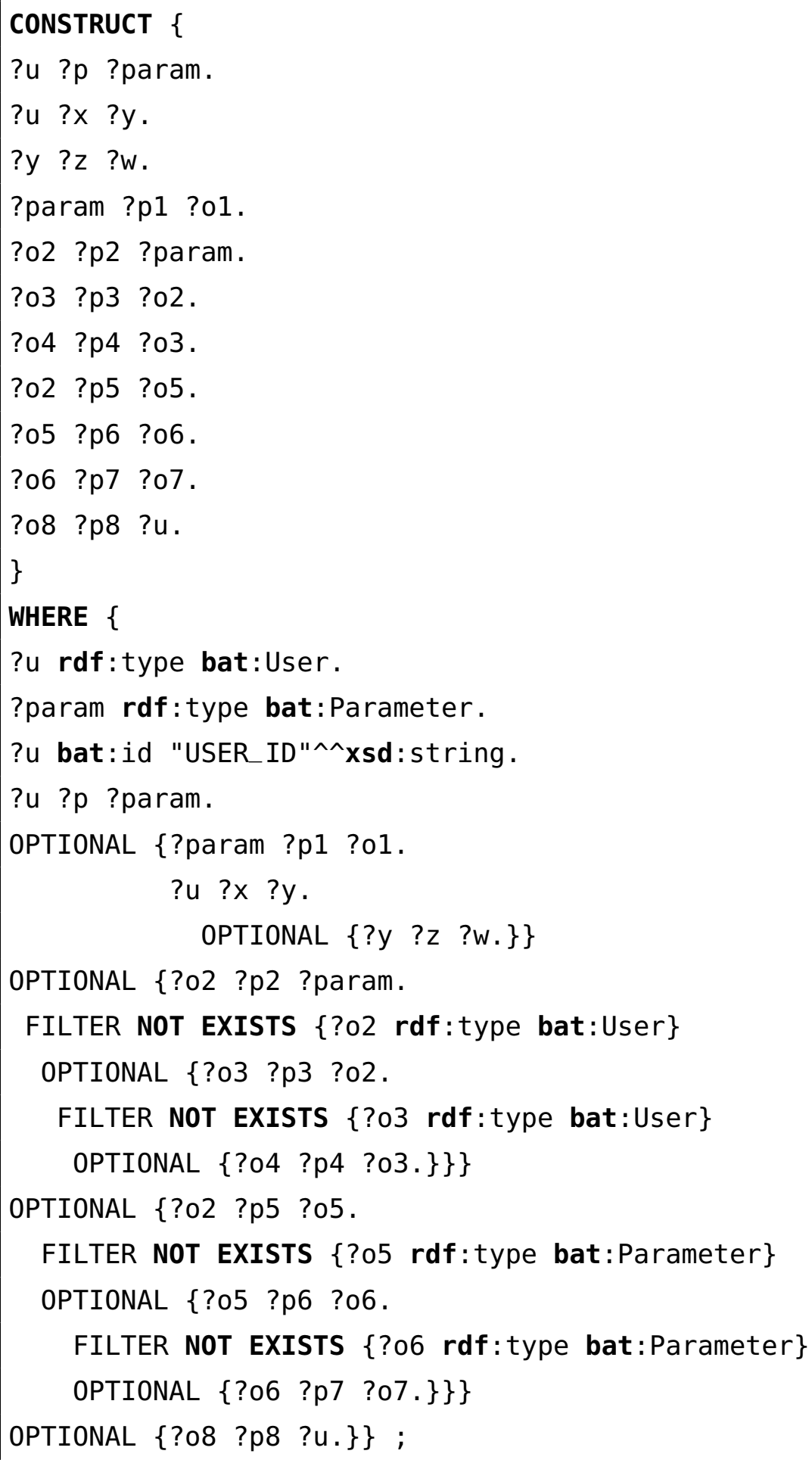




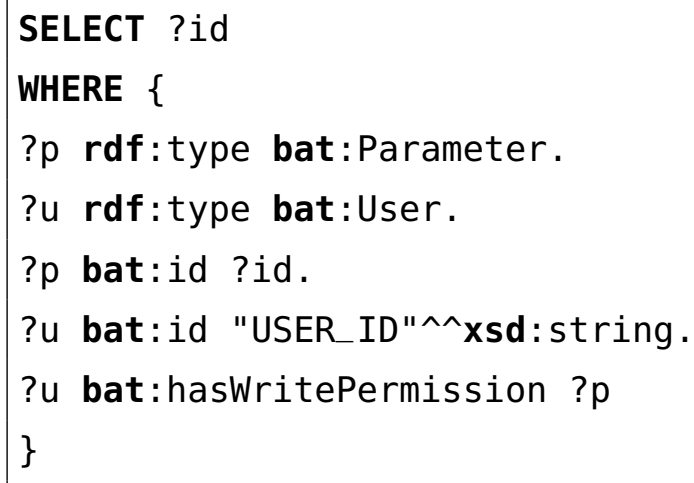

Listing 5.4 SPARQL query for all the parameters that the user can modify

\subsubsection{Control of expiration of tokens}

All the generated PKGs are stored in different maps in the Security Manager. Every time a login action is performed, a new entry $<$ Token, $\mathrm{PKG}>$ is added into either the map TokenAdminPKG or Token-NonAdminPKG. All the entries are stored in the RAM for the quick access. Meanwhile, the token can be used persistently even if sometimes the human being who is controlling has changed. As a result, considering the security and memory issue, Security Manager is required to remove the inactive PKGs regularly:

- Each token is associated with a timestamp, which indicates the last time when the PKG of this token was accessed. The timestamp is stored in the form of millisecond value that is an offset from the Epoch.

- All the entries < Token, timestamp $>$ are stored in the map Token-TimeStamp of Authorization Validator and are updated when a request containing this token is received

- The expiration time of a token is configurable in milliseconds. Assuming that the expiration time is set as X, then every X/2 milliseconds, Authorization Validator checks the timestamp of each token.

- If a token has been inactive for more than X milliseconds, all the entries that are related with this token should be removed from the related maps, which are:

- Token-AdminModel

- Token-NonAdminModel

- Token-NonAdminUserID

- Token/BorderRouterID-ClientID 
- Token WritableParameterList

- Token-TimeStamp

\subsubsection{On-the-fly updates of PKGs}

For each client, only the administrator can modify the configuration of this client, including creating/delete users, roles, spaces devices as well as associating roles to users, devices to spaces. The change of the configuration could affect the PKGs of the non-administrator users that are currently logged in. Therefore, as long as an administrator performs a logout action and changes are detected in the named graph of this client, the Inference Manager should launched again the inference and all the PKGs for the non-administrator users of the same client should be regenerated and updated.

As the example shown in Fig. 5.1 and Fig. 5.7, both userA and userB belong to client0001, while userA is administrator. When userA logout out, a logout request with Token-1 in the Authorization header is received by the platform:

[1] By consulting the Authorized PKG Manager, Token-1 is in the map Token-AdminPKG, which indicates that the user performing logout is an administrator.

[2] By consulting the map Token/BorderRouterID-ClientID, the corresponding clientID for Token-1 is client0001

[3] Check the map ClientID-changed, the changed flag corresponding to client0001 has been set to true

[4] An inference is launched against the NG-client0001.

[5] According to the maps Token/BorderRouterID-ClientID, all Token-1, Token-2 and BorderRouter1 are corresponding to client0001.

[6] The Security Manager regenerates the PKG-userA, PKG-userB and PKG-BorderRouter1 and replace the old PKGs with the new ones in each map. 



\section{Chapter 6}

\section{Case study and test bed}

In this chapter, a case study will be presented to explain in detail the working mechanism of the developed authorization control system. Meanwhile, a test-bed has been implemented to evaluate the performance of the developments done in this thesis that include the ontology BATOnt, Web API and the authorization mechanism.

\subsection{Case study}

The purpose of this case study is to provide a real situation to assess working process of the developed access control mechanism in the platform and to test the inference with the defined rules. The example is a simplified case, and to protect the user privacy, all the entities are denoted with invented IDs and names instead of real user information. Table 6.1 shows the definition of namespaces in this section.

\begin{tabular}{|c||c|}
\hline Prefix & URL \\
\hline rdf & http://www.w3.org/1999/02/22-rdf-syntax-ns\# \\
\hline rdfs & http://www.w3.org/2000/01/rdf-schema\# \\
\hline xs & http://www.w3.org/2001/XMLSchema\# \\
\hline bat & www.cedint.upm.es/bat.owl\# \\
\hline ex & www.example.com\# \\
\hline
\end{tabular}

Table 6.1 Definition of namespaces

In the System KG, all the KGs and PKGs are stored in the form of semantic language W3C Web Ontology Language (OWL), which is the machine-readable form of ontologies. In this case study, all the KGs including the Original KG, Deduction KG and PKGs have been 
exported in the form of OWL documents to show their content. To concentrate on the main part of the KG, all the OWL files shown in this section has been simplified by removing the non-essential properties.

\subsubsection{Overview of KG for the client}

To illustrate the process of System KG construction, inference, generation of PKGs and Web API a real scenario has been used.

A client with ID "client0001" has been created. A client includes the information about users, spaces and devices.

Users can belong to the following groups:

- Software group (SW)

- Hardware group (HW)

A user can belong to one or to both groups.

Accordingly, all the instances that are related to the client are stored in the named graph "batmp.cedint.upm.es/BATOnt/client0001" and the deductions are stored in the named graph "batmp.cedint.upm.es/BATOnt/inf/client0001". Fig. 6.1 illustrates the overview of the knowledge graph for client0001.

The KG includes seven classes (bat:Client, bat:User, bat:Role, bat:ParameterType, bat:Parameter, bat:Device, and bat:Space), twenty instances (ex:Client0001, ex:User1, ex:User2, ex:User3, ex:RoleSW, ex:RoleHW, bat:TemperatureCelsius, bat:OnOff, ex:RealDaikinTemperatureSW, ex:RealDaikinTemperatureHW, ex:SetTemperatureSW, ex:SetTemperatureHW, ex:BATTemperature, ex:BatPlug-state_OnOff, ex:SWDaikin, ex:HWDaikin, ex:HWBATSense, ex:BatPlug, ex:OfficeSW, ex:OfficeHW ), object and datatype properties (marked with arrows). Instances in this $\mathrm{KG}$ can be explained from four points of view:

With respect to the administration There have been created:

- Three non-administrator users: ex:User1, ex:User2, ex:User3, and

- Two roles: ex:RoleSW and ex:RoleHW.

- The roles have been assigned to users as follows (see Fig. 6.1):

- ex:User1 has ex:RoleSW

- ex:User2 has ex:RoleSW and ex:User2 has ex:RoleHW 


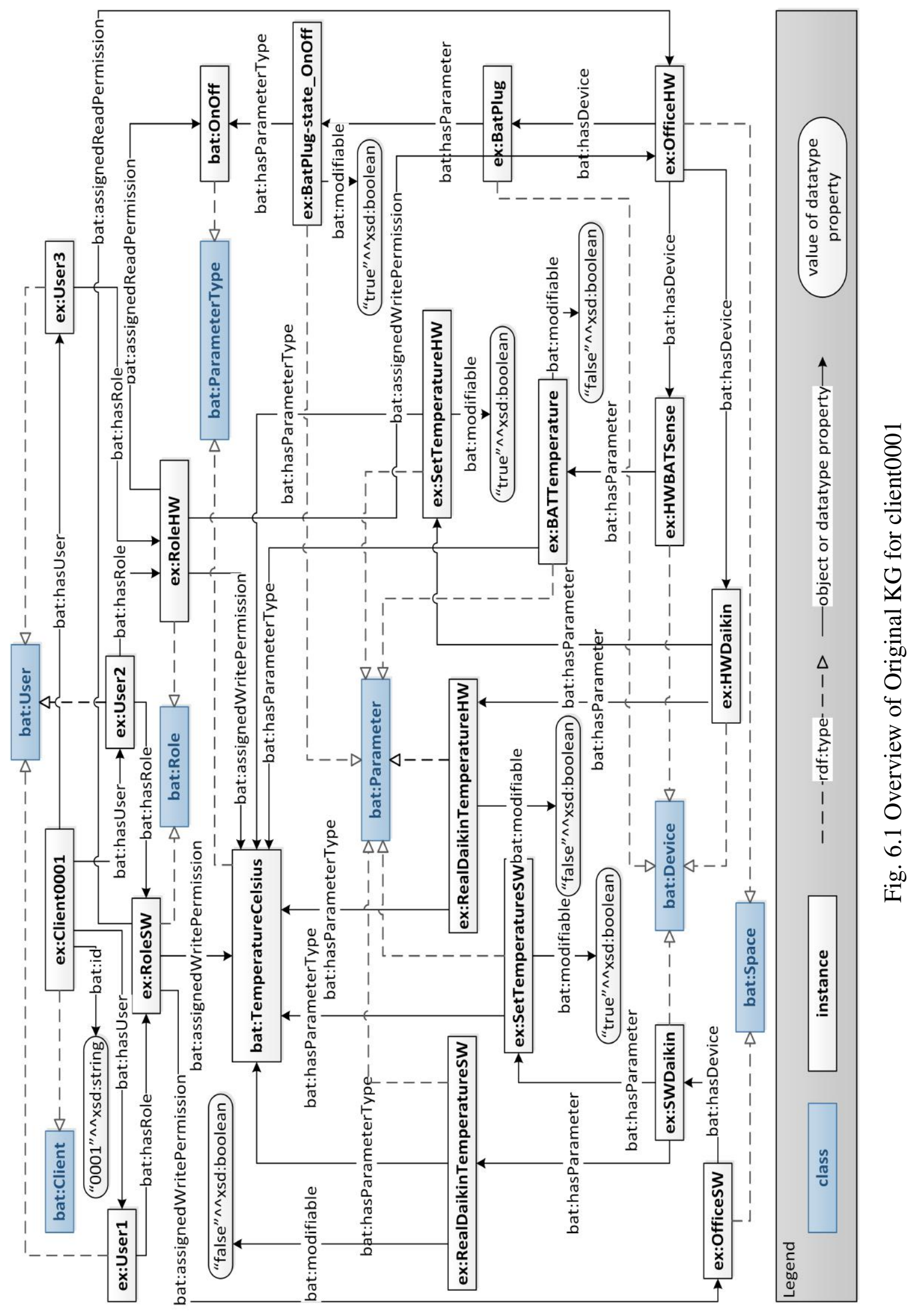


- ex:User3 has ex:RoleHW

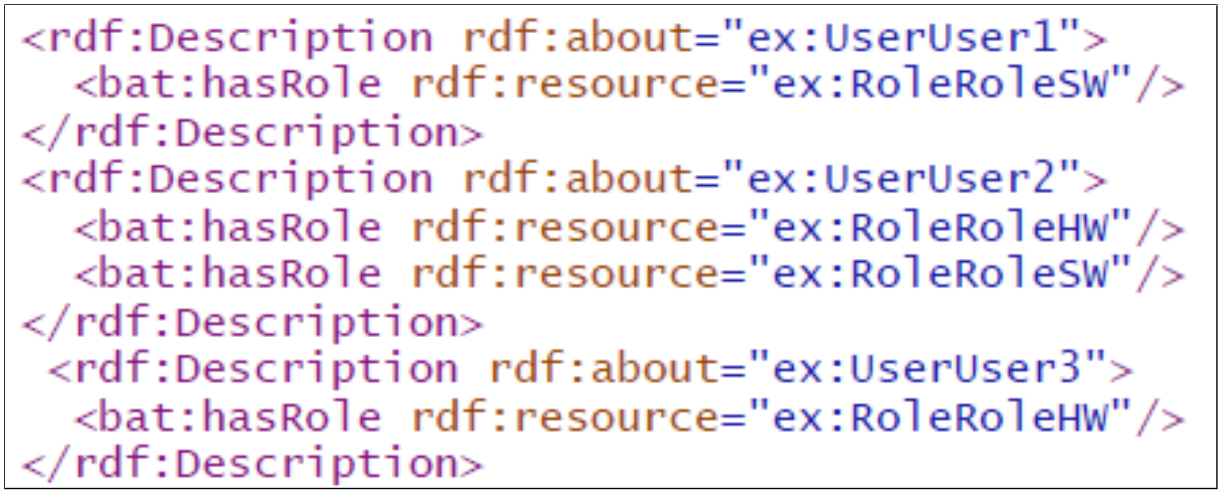

Fig. 6.2 Statements about administration in OWL file

Fig. 6.2 shows part of the OWL file of the Original KG, which includes the statements regarding the administration.

With respect to the Devices Four devices have been created:

- ex:SWDaikin and ex:HWDaikin, that corresponds with two HVAC systems. [127]

- ex:HWBATSense, is a WTN device to measure indoor ambient parameters.

- ex:BatPlug, is a WTN device for smart plug.

The parameters of each device are the following (see Fig. 6.1).

- ex:SWDaikin has parameters ex:RealDaikinTemperatureSW and ex:SetTemperatureSW

- ex:HWDaikin has parameters ex:RealDaikinTemperatureHW and ex:SetTemperatureHW.

- ex:HWBATSense has parameter ex:BATTemperatureHW

- ex:BatPlug has parameter ex: BatPlug-state-OnOff.

The parameter type of each parameter are the following:

- ex:RealDaikinTemperatureSW has parameter type bat:TemperatureCelsius

- ex:SetTemperatureSW has parameter type bat:TemperatureCelsius

- ex:RealDaikinTemperatureHW has parameter type bat:TemperatureCelsius 
- ex:SetTemperatureHW has parameter type bat:TemperatureCelsius

- ex:BATTemperatureHW has parameter type bat:TemperatureCelsius

- ex:BatPlug-state-OnOff has parameter type bat:OnOff.

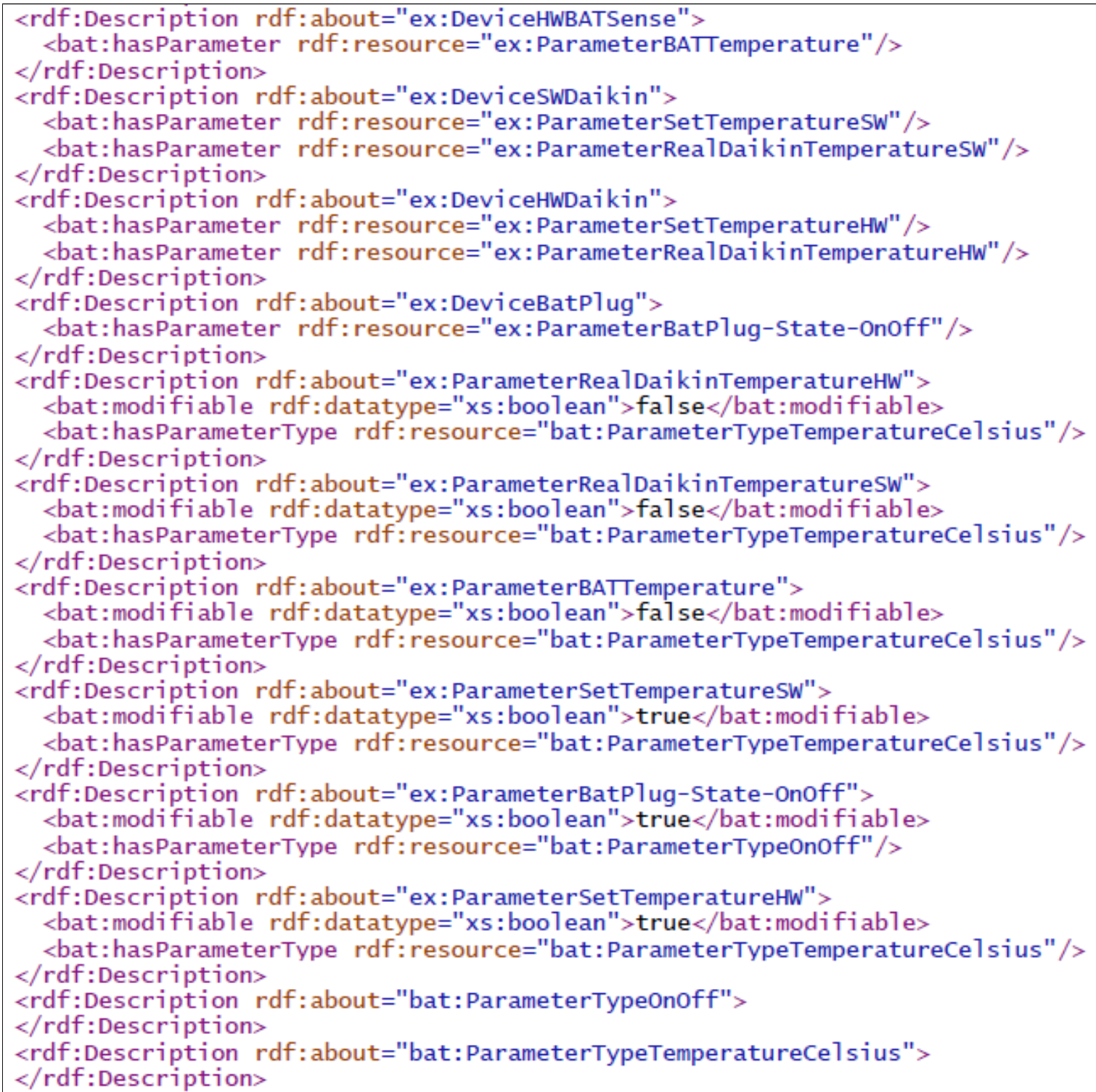

Fig. 6.3 Statements about Things in OWL file

Fig. 6.3 shows part of the OWL file of the Original KG, which includes the statements regarding the devices. 
With respect to building layout Two spaces have been created:

- ex:OfficeSW

- ex:OfficeHW

Devices belong to spaces as follows:

- ex:SWDaikin is inside ex:OfficeSW

- ex:HWDaikin, ex:HWBATSense and ex:BatPlug are inside ex:OfficeHW

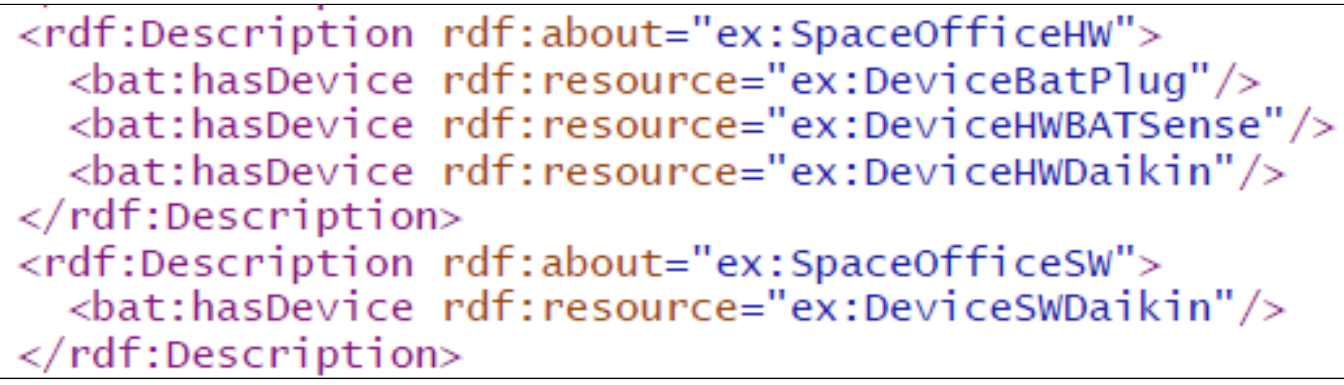

Fig. 6.4 Statements about building layout in OWL file

Fig. 6.4 shows part of the OWL file of the Original KG, which includes the statements regarding the building layout.

With respect to trust policies Six relationships have been set.

- ex:RoleSW is assigned Write permission to ex:OfficeSW

- ex:RoleSW is assigned Read permission to ex:OfficeHW

- ex:RoleHW is assigned Write permission to ex:OfficeHW

- ex:RoleSW is assigned Write permission to bat:TemperatureCelsius

- ex:RoleHW is assigned Read permission to bat:OnOff

- ex:RoleHW is assigned Write permission to bat:TemperatureCelsius 


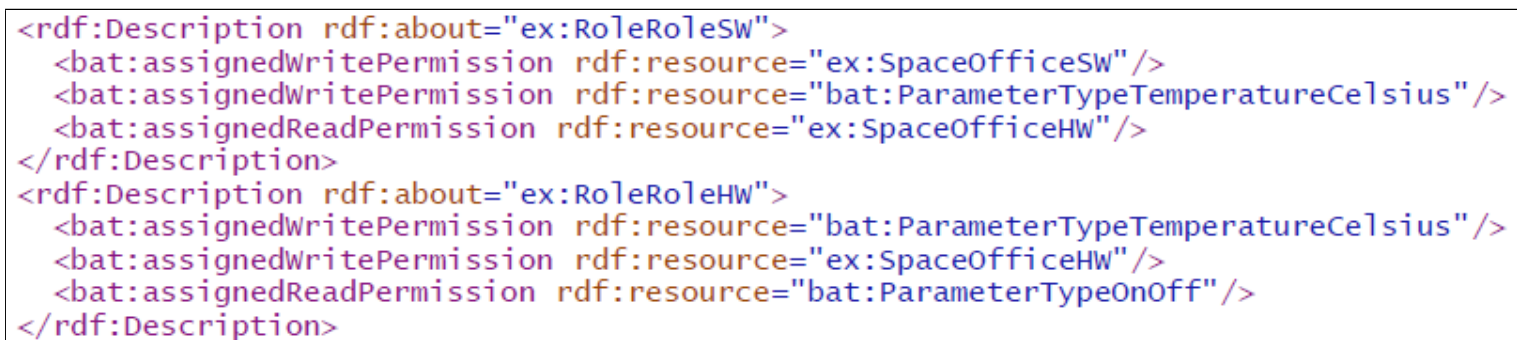

Fig. 6.5 Statements about trust policies in OWL file

Fig. 6.5 shows part of the OWL file of the Original KG, which includes the statements regarding the trust policies.

\subsubsection{Perform inference with fact rules}

On the basis of the Original KG presented above, by performing the inference with the fact rules introduced in Chapter 4, the implied relationships between the spaces and parameters can be retrieved, as illustrated in Fig. 6.6

Six inferred object properties are obtained, which are:

- ex:OfficeSW bat:hasParameter ex:RealDaikinTemperatureSW

- ex:OfficeSW bat:hasParameter ex:SetTemperatureSW

- ex:officeHW bat:hasParameter ex:RealDaikinTemperatureHW

- ex:officeHW bat:hasParameter ex:SetTemperatureHW

- ex:officeHW bat:hasParameter ex:BATTemperature

- ex:officeHW bat:hasParameter ex:BatPlug-state-OnOff

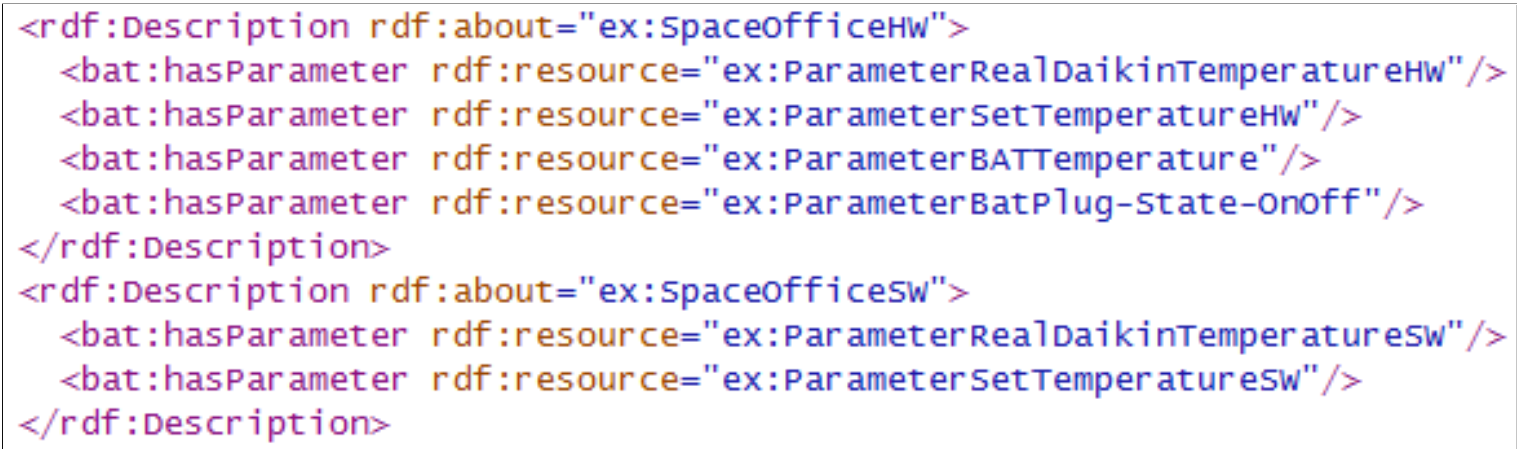

Fig. 6.7 Statements obtained by applying fact rules in OWL file 


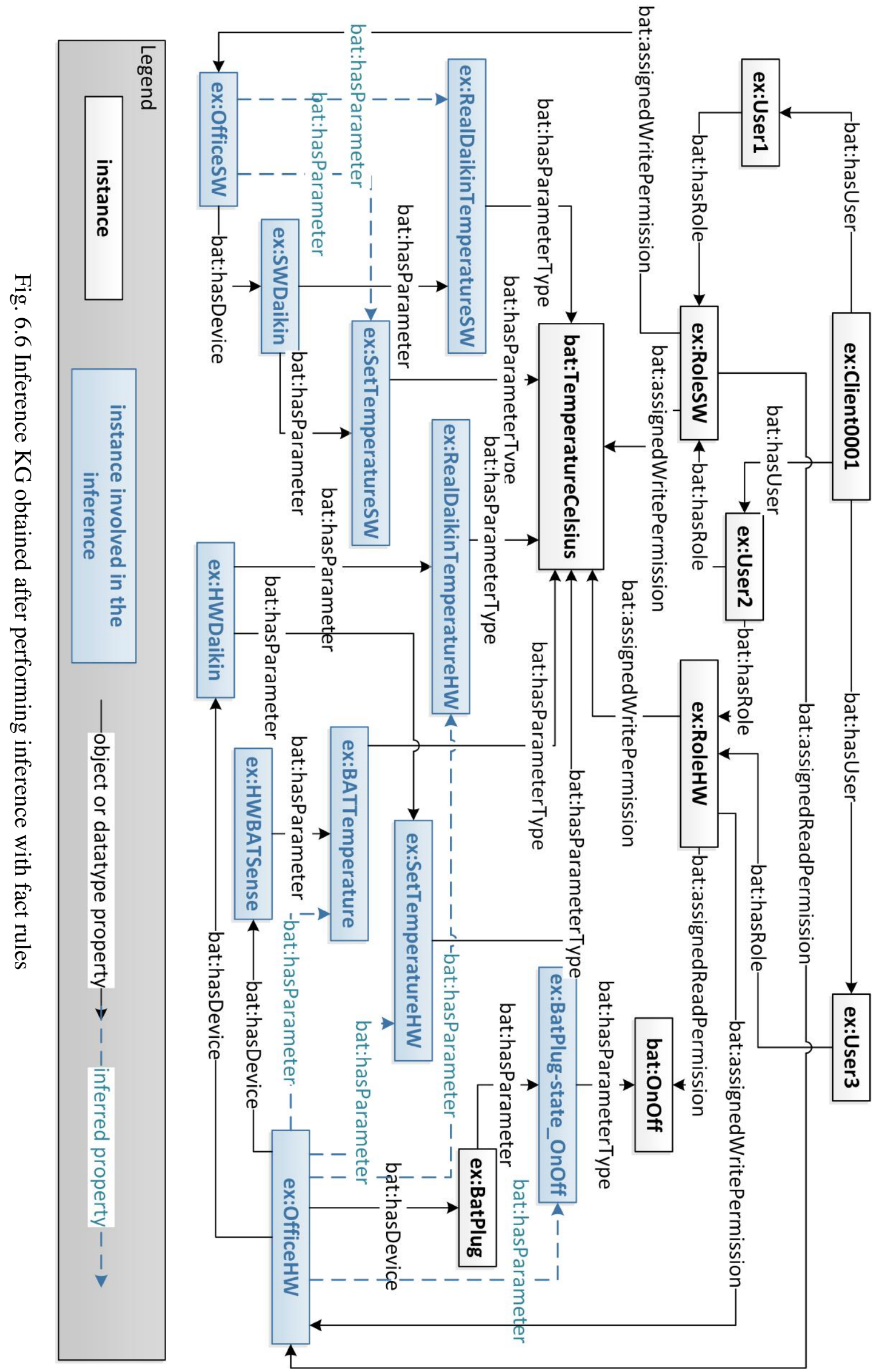


Fig. 6.7 shows part of the OWL file of the Deductions KG, which includes the statements that have been obtained by applying inference with the fact rules.

\subsubsection{Perform inference with policy rules and generate PKGs}

On the basis of the KG obtained above, the permission relationships among users, roles and parameters can be obtained by performing inference with the policy rules introduced in Chapter 4. The following sections will explain respectively the inferred object properties for User1, User2 and User3, as illustrated in Fig. 6.8, Fig. 6.11 and Fig. 6.14. All the entities related to the corresponding user are highlighted in blue and the inferred properties are denoted by coloured dashed lines. The red dashed lines represent the inferred properties obtained by applying the policy rules for Role-Parameter permissions, and the blue dashed lines represent the inferred properties obtained by applying the policy rules for User-Parameter permissions, which are also actually the ultimate access rights assigned to the users.

\section{PKG for user1}

Fig. 6.8 shows the structure of PKG for ex:User1, who has only one role, ex:RoleSW.

As it is illustrated in the figure, ex:RoleSW is assigned Read permission to the space ex:OfficeHW. Meanwhile, ex:RoleSW is assigned Write permission to the parameter type bat:TemperatureCelsius and the space ex:OfficeSW. As a result, after the inference, the following deductions can be obtained.

By applying Role-Parameter permission rules Five deductions are obtained:

- ex:RoleSW has Read permission to ex:RealDaikinTemperatureSW

- ex:RoleSW has Read permission to ex:RealDaikinTemperatureHW

- ex:RoleSW has Read permission to ex:SetTemperatureHW

- ex:RoleSW has Read permission to ex:BATTemperature

- ex:RoleSW has Write permission to ex:SetTemperatureSW 


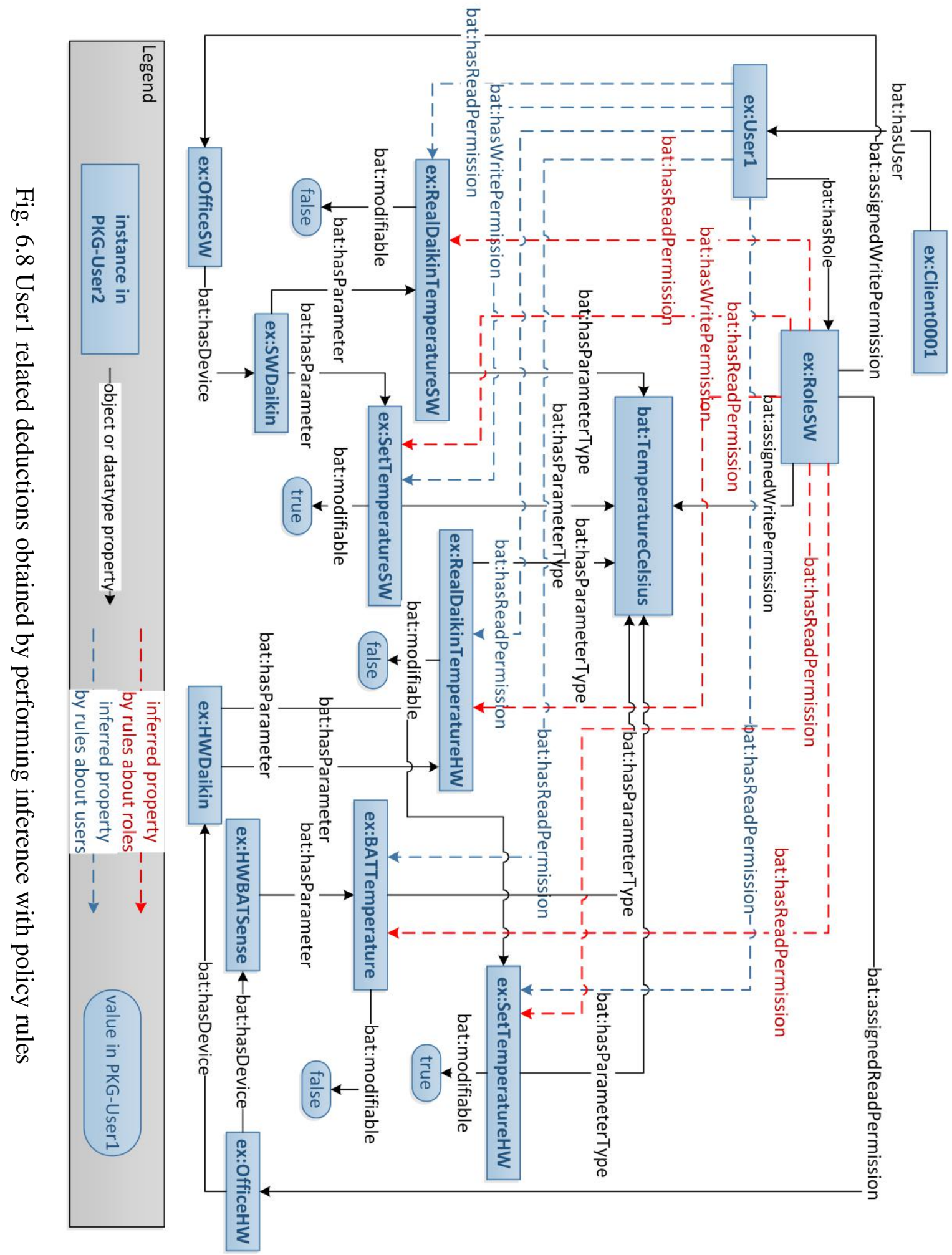




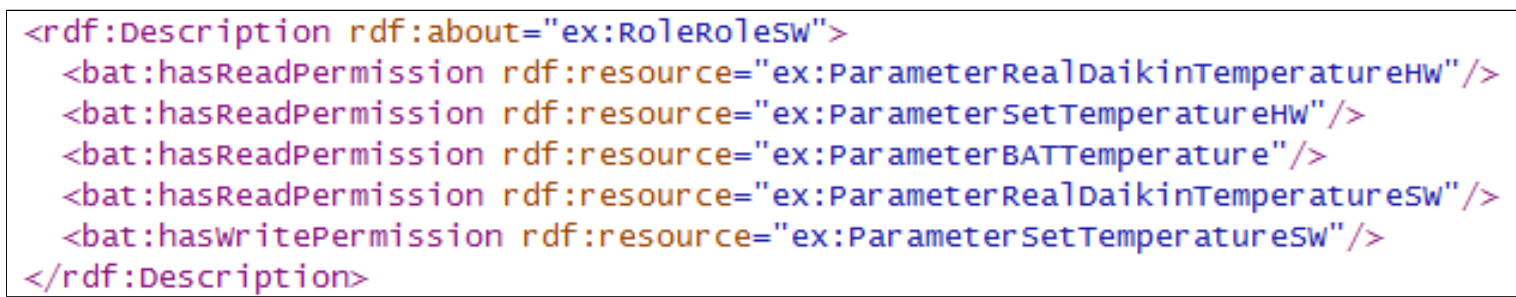

Fig. 6.9 Statements related to ex:RoleSW obtained by applying policy rules in OWL file

By applying User-Parameter permission rules User ex:User1 should have all the permissions that its role ex:RoleSW has, therefore:

- ex:User1 has Read permission to ex:RealDaikinTemperatureSW

- ex:User1 has Read permission to ex:RealDaikinTemperatureHW

- ex:User1 has Read permission to ex:SetTemperatureHW

- ex:User1 has Read permission to ex:BATTemperature

- ex:User1 has Write permission to ex:SetTemperatureSW

Fig. 6.10 shows part of the OWL file of the Deductions KG, which includes the statements related to ex:User1 that have been obtained by applying the policy rules.

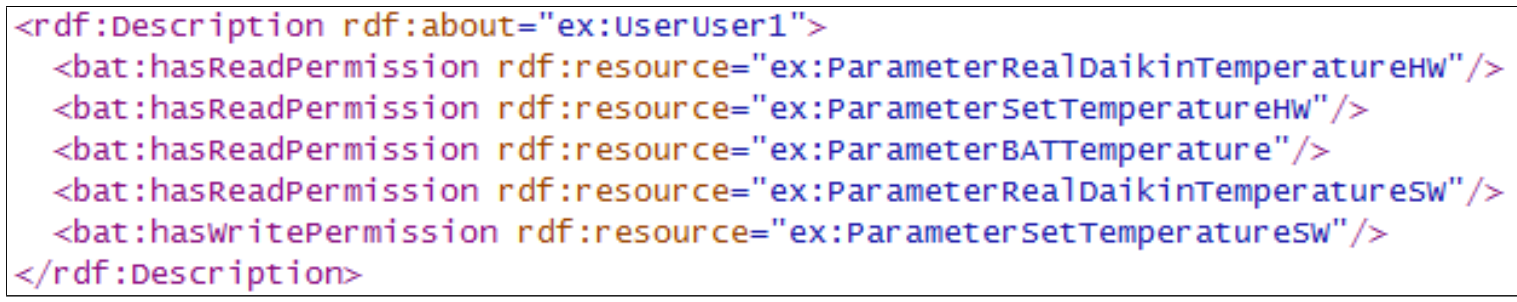

Fig. 6.10 Statements related to ex:User1 obtained by applying policy rules in OWL file

The PKG generated for User1 is denoted as PKG-User1 and consists of all the entities highlighted in blue.

\section{PKG for user2}

Fig. 6.11 shows the structure of PKG diagram for ex:User2.

As illustrated in the figure, ex:User2 has two roles, ex:RoleSW and ex:RoleHW. While ex:RoleHW is assigned Read permission to parameter type bat:OnOff. Meanwhile, ex:RoleHW is assigned Write permission to the parameter type bat:TemperatureCelsius and the space ex:OfficeHW. By performing the inference, the following deductions can be obtained: 


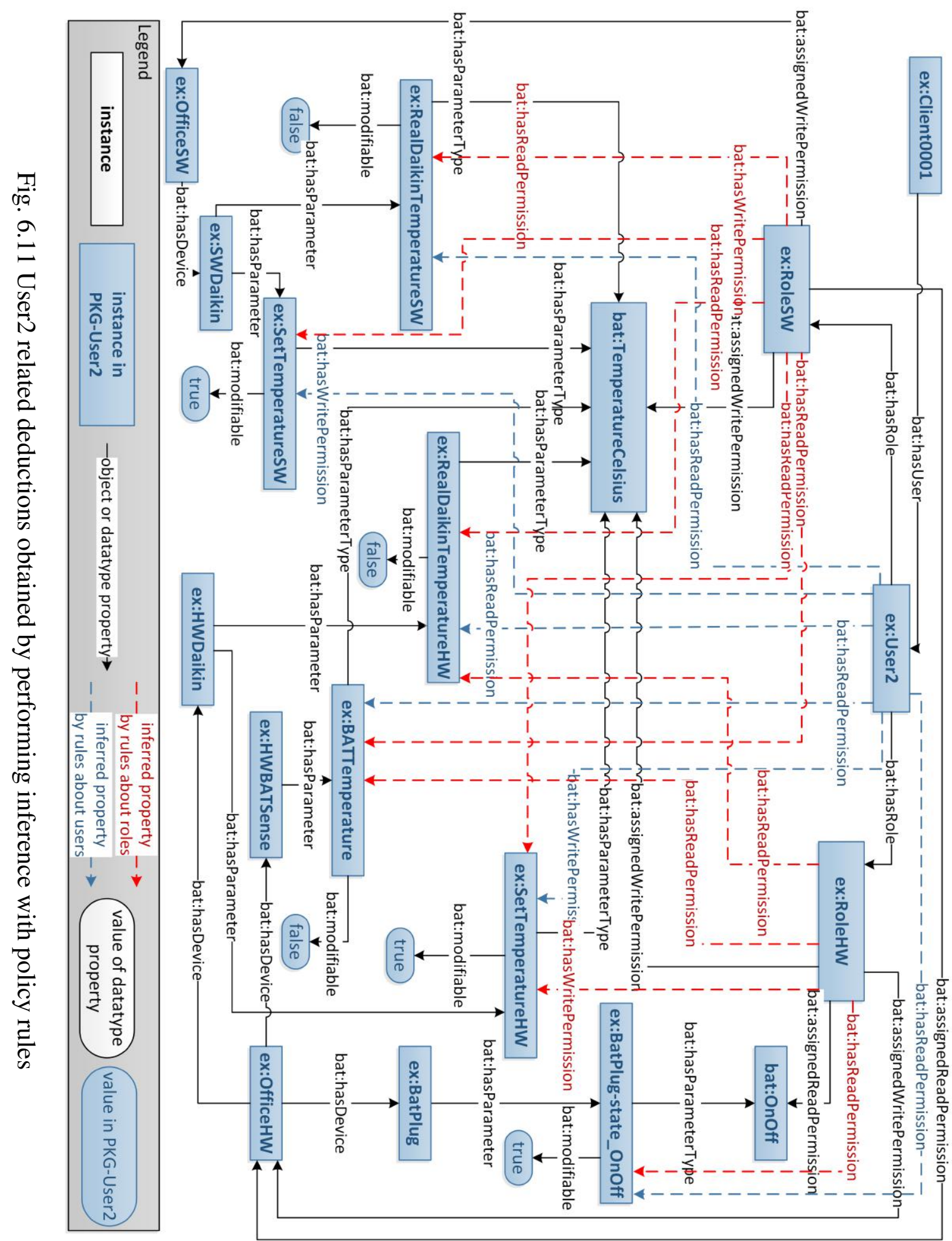


By applying Role-Parameter permission rules Apart from the five deductions obtained for ex:RoleSW, which have been introduced above, four more deductions are obtained for ex:RoleHW:

- ex:RoleHW has Read permission to ex:RealDaikinTemperatureHW

- ex:RoleHW has Read permission to ex:BATTemperature

- ex:RoleHW has Read permission to ex:BatPlug-state_OnOff

- ex:RoleHW has Write permission to ex:SetTemperatureHW

Fig. 6.12 shows part of the OWL file of the Deductions KG, which includes the statements related to ex:RoleHW that have been obtained by applying the policy rules.

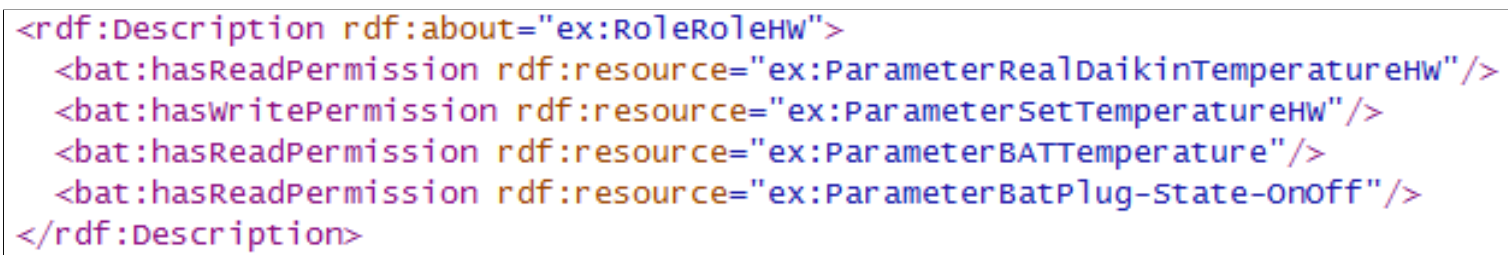

Fig. 6.12 Statements related to ex:RoleHW obtained by applying policy rules in OWL file

By applying User-Parameter permission rules User ex:User2 should has all the access rights that ex:RoleSW and ex:RoleHW holds, therefore:

- ex:User2 has Read permission to ex:RealDaikinTemperatureSW

- ex:User2 has Read permission to ex:RealDaikinTemperatureHW

- ex:User2 has Read permission to ex:BATTemperature

- ex:User2 has Read permission to ex:BatPlug-state_OnOff

- ex:User2 has Write permission to ex:SetTemperatureSW

- ex:User2 has Write permission to ex:SetTemperatureHW

Fig. 6.13 shows part of the OWL file of the Deductions KG, which includes the statements related to ex:User2 that have been obtained by performing inference with the policy rules. 


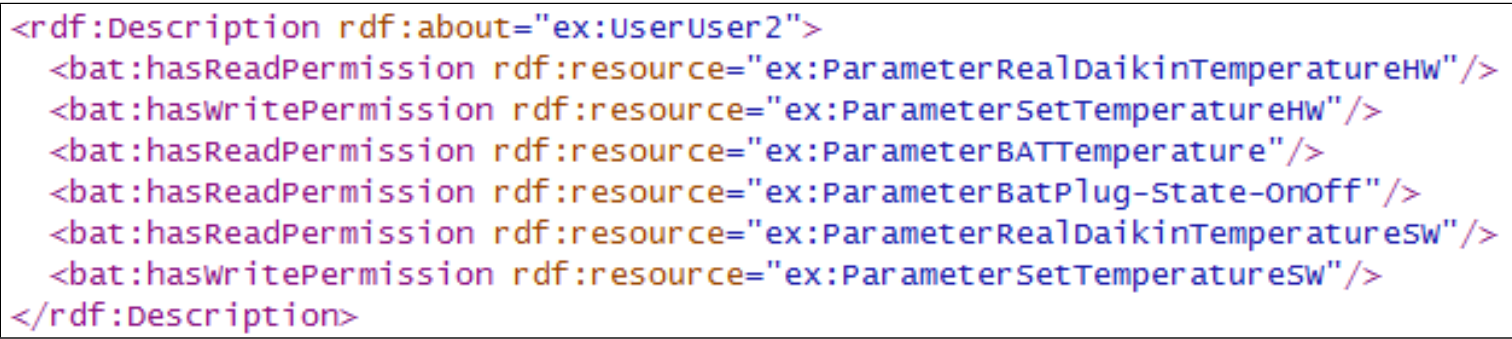

Fig. 6.13 Statements related to ex:User2 obtained by applying policy rules in OWL file

The PKG generated for User2 is denoted as PKG-User2 and consists of all the entities highlighted in blue.

\section{PKG for user3}

Fig. 6.14 shows the structure of PKG for User3. User ex:User3 has only one role, ex:RoleHW.

The deductions for ex:RoleHW have been explained above. Since ex:User3 only has one role, he has exactly the same permissions that ex:RoleHW does. By applying User-Parameter permission rules, the following deductions are obtained:

- ex:User3 has Read permission to ex:RealDaikinTemperatureHW

- ex:User3 has Read permission to ex:BATTemperature

- ex:User3 has Read permission to ex:BatPlug-state_OnOff

- ex:User3 has Write permission to ex:SetTemperatureHW

Fig. 6.15 shows part of the OWL file of the Deductions KG, which includes the statements related to ex:User3 that have been obtained by applying the policy rules.

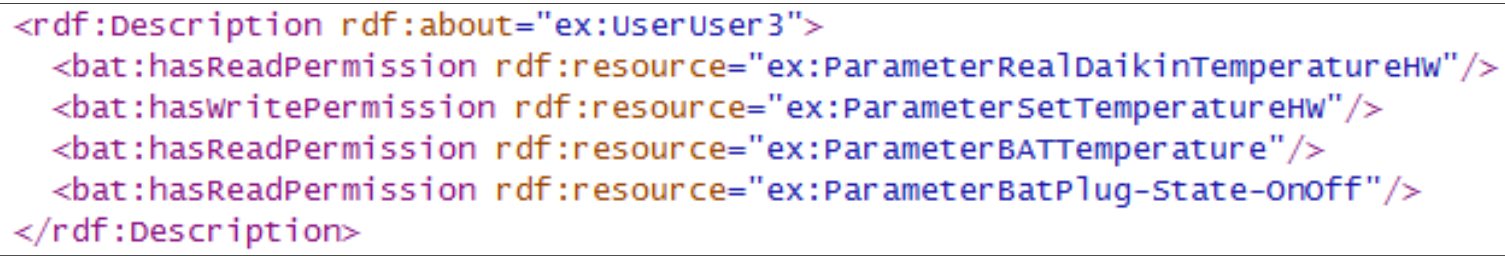

Fig. 6.15 Statements related to ex:User3 obtained by applying policy rules in OWL file

The PKG generated for User3 is denoted as PKG-User3 and is formed by all the entities highlighted in blue. Fig. 6.16 shows the OWL file of the PKG-User3. 


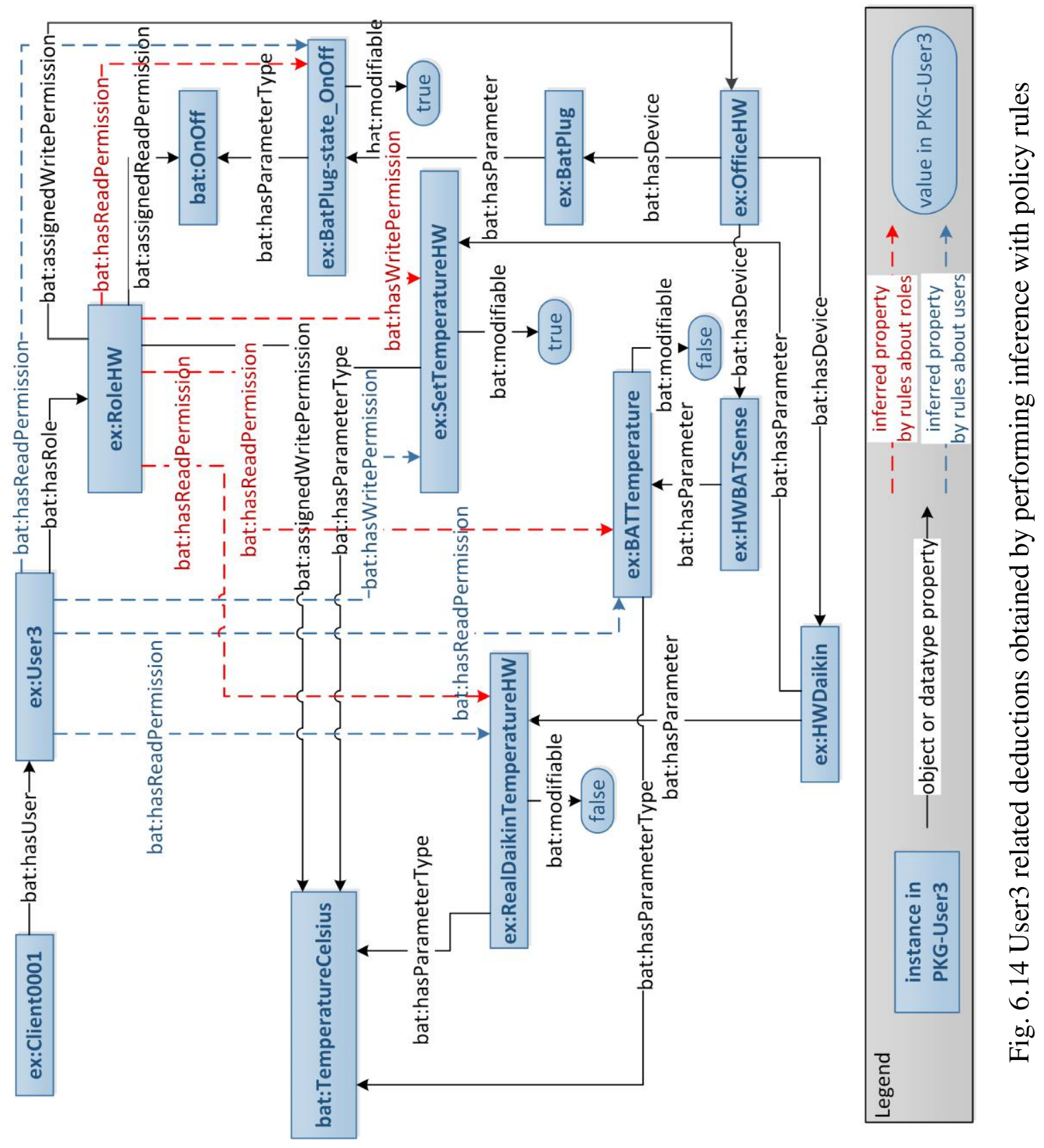




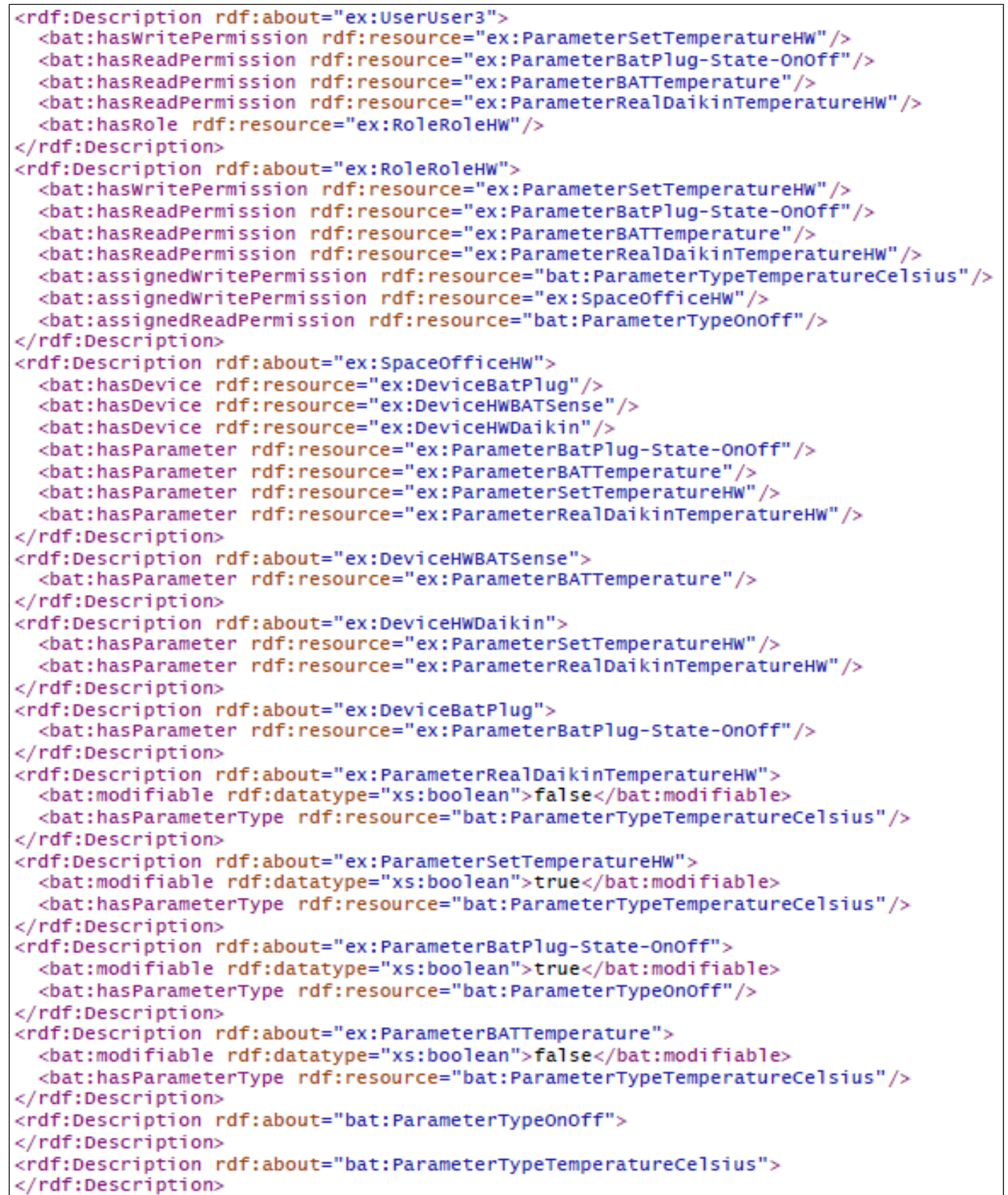

Fig. 6.16 PKG-User3 in OWL file

As it is shown in this document, the PKG generated for User3 contains only the information that he is authorized to access, including:

- The basic user information of ex:User3

- The roles that belong to ex:User3 
- The spaces that ex:User3 has right to access

- The parameters that can be read or modified by ex:User3

- The devices to which those parameters belong and the parameter types that belong to those parameters

- All the relationships among the entities mentioned above

\subsection{Test bed}

In order to assess the experimental work done in this thesis it is necessary to evaluate the following aspects:

- To provide a guidance on using the created ontology BATOnt in practical projects.

- The performance of the authentication and authorization mechanisms developed.

- To verify the capability of the IoT platform to work with different communication protocols.

- To check the performance of the designed Web API in a real environment.

- Demonstrate the use of existing applications (3D Virtual Reality and WeChat) to access IoT devices through the Web API developed.

\subsubsection{Overview of facilities}

The test bed has been implemented based on a show-room available at the Research Centre for Smart Buildings and Energy Efficiency (CeDInt) of Universidad Politécnica de Madrid (UPM). The show-room is equipped with commercial home automation and industrial technologies: KNX, Modbus, LonWorks, X10, EnOcean and ZigBee. Deployed devices include ambient sensors, HVAC system, energy meter, lighting control system, blind actuators and the BATNet WTN devices developed by CeDInt. Also a mini-greenhouse has been used for testing purposes. Fig. 6.17 shows the view of the CeDInt showroom and the mini greenhouse. 


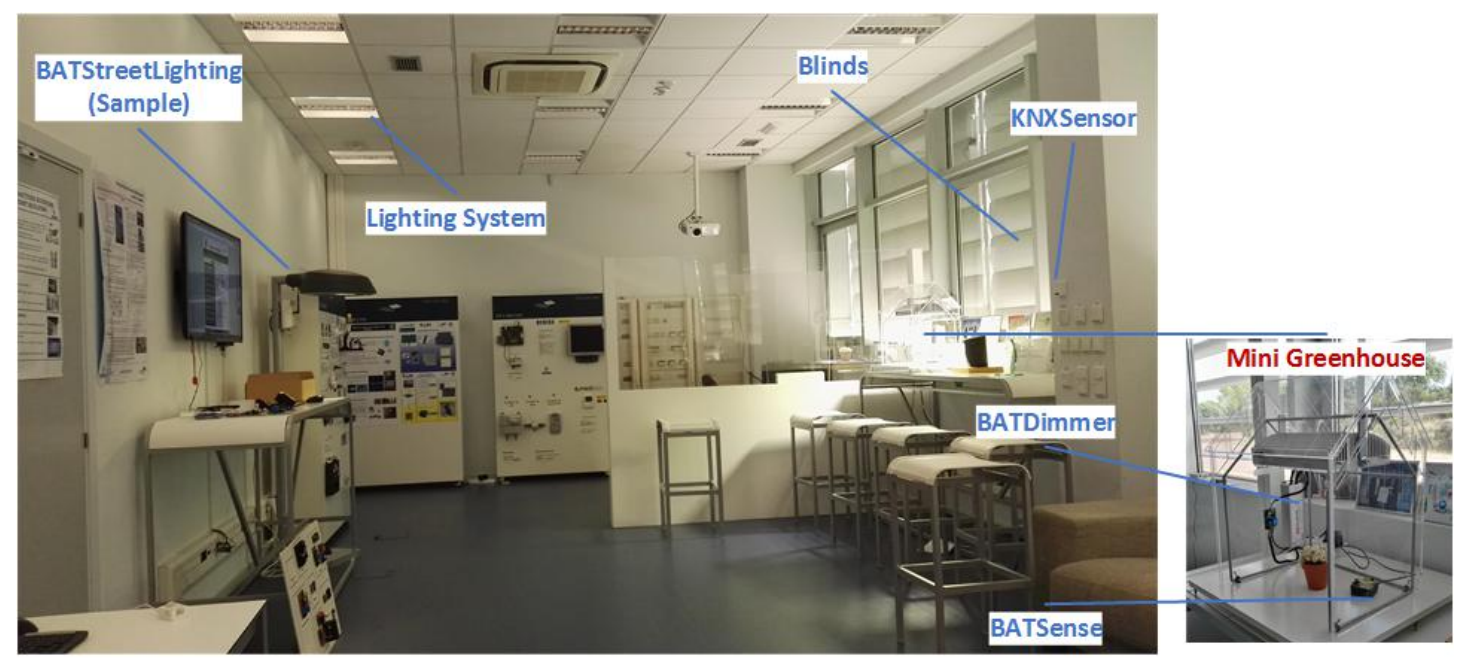

Fig. 6.17 View of showroom in CeDInt

The devices used in this test bed are the following:

- Blind Actuator: This device controls the \% of aperture of blinds. Four blinds can be controlled with a single device using the group addresses.

- Light Actuator: This device controls de \% of dimming of lights. Three lights (or group of lights) can be controlled with a single device using the group addresses.

- KNX Sensor 108: This device measures indoor ambient temperature and humidity.

- Weather Station CEDINT: This devices measures outdoor temperature, rain, sun position, illumination and wind force.

- BATStreetLighting BSL01-0036: This device measures the outdoor illumination level and controls the $\%$ of dimming of light.

- BATSense_minigreenlab: This device measures Temperature, Humidity, Illumination, Battery and Presence in indoor environments.

- BATDimmer_minigreenlab: This device controls de \% of dimming of lights.

Table 6.2 shows the devices characteristics. 


\begin{tabular}{|c|c|c|c|c|}
\hline Device Name & Device ID & Device Type & Driver & Space \\
\hline BlindActuator & nserie-glharg45 & Blind Actuator & KNXDriver & CEDINT \\
\hline LightActuator & nserie-fasdfa45 & Light Actuator & KNXDriver & CEDINT \\
\hline KNX Sensor 108 & KNXTHER108110 & Thermostat Sensor & KNXDriver & CEDINT \\
\hline Weather Station CEDINT & WEATHERSTATION1 & Weather Station & KNXDriver & N/A \\
\hline BATStreetLighting BSL01-0036 & BSL01-0036 & BatStreetLighting & BATNetDriver & CEDINT \\
\hline BATSense_minigreenlab & BSN01-0042 & BATSense & BATNetDriver & MiniGreenhouse \\
\hline BATDimmer_minigreenlab & BDM01-0041 & BATDimmer & BATNetDriver & MiniGreenhouse \\
\hline
\end{tabular}

Table 6.2 Devices used in the test bed

Regarding the communication technology the test bed includes:

- Four KNX devices(BlindActuator, LightingActuator, KNX Sensor 108 and Weahter Station CEDINT)

- Three BATNet devices(BATSreetLighting BSL01-0036, BATSense_miigreenlab and BATDimmer_minigreenlab)

Regarding the spaces, the test bed includes two spaces:

- CEDINT. There are four devices belonging to CEDINT space: BlindActuator, LightActuator, KNXsensor 108 and BATStreetLighting BSL01-0036.

- MiniGreenhouse. There are two devices belonging to MiniGreenhouse space: BATSense _minigreenlab and BATDimmer_minigreenlab.

It must be noticed that Weather Station CEDINT device does not belong to any space. This has been intentionally done to demonstrate that there can be devices not associated to spaces. In these cases only the Administrator can read and write on them.

In the system, there are in total twenty-seven parameters created for the seven devices.

Table.6.3 shows detailed information (parameter IDs, parameter type, data type, modifiable and access type) about devices belonging to CEDINT Space. 


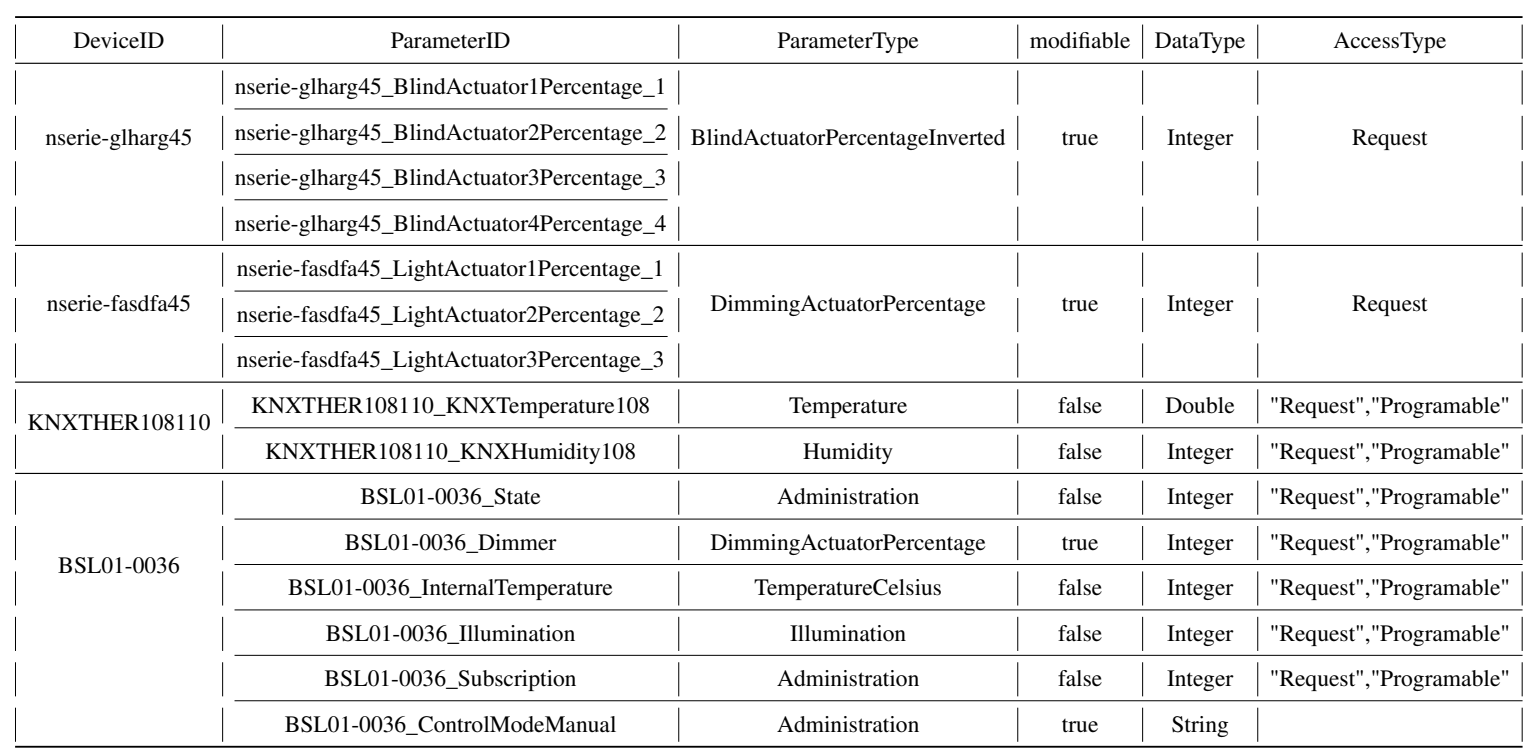

Table 6.3 Parameters of devices in CEDINT Space

Table.6.4 shows detailed information (parameter IDs, parameter type, data type, modifiable and access type) about devices belonging to MiniGreenHouse Space.

\begin{tabular}{|c|c|c|c|c|c|}
\hline DeviceID & ParameterID & ParameterType & modifiable & DataType & AccessType \\
\hline \multirow{4}{*}{ BSN01-0042 } & BSN01-0042_Battery & BatteryVoltage & false & Integer & "Request","Programable" \\
\cline { 2 - 6 } & BSN01-0042_Temperature & TemperatureCelsius & false & Double & "Request","Programable" \\
\cline { 2 - 6 } & BSN01-0042_Illumination & IlluminationLevel & false & Integer & "Request","Programable" \\
\cline { 2 - 6 } & BSN01-0042_Humidity & Humidity & false & Integer & "Request","Programable" \\
\cline { 2 - 6 } & BSN01-0042_Presence & Presence & false & Integer & "Request","Programable" \\
\hline BDM01-0041 & BDM01-0041_Dimmer & DimmingActuatorPercentage & true & Integer & "Request","Programable" \\
\hline
\end{tabular}

Table 6.4 Parameters of devices in MiniGreenhouse Space

Table.6.5 shows the details of device Weather Station CEDINT, that does not belong to any space.

\begin{tabular}{|c|c|c|c|c|}
\hline DeviceID & ParameterID & ParameterType & modifiable & AccessType \\
\hline \multirow{5}{*}{ WEATHERSTATION1 1} & WEATHERSTATION1_Illumination & IlluminationLevel & false & "Request","Programable" \\
\cline { 2 - 5 } & WEATHERSTATION1_SunPositionElevation & SolarElevationAngle & false & "Request","Programable" \\
\cline { 2 - 5 } & WEATHERSTATION1_SunPositionAcimut & SolarAzimuthAngle & false & "Request","Programable" \\
\cline { 2 - 5 } & WEATHERSTATION1_RainStatus & RainStatus & false & "Request","Programable" \\
\cline { 2 - 5 } & WEATHERSTATION1_WindForce & WindSpeedKMPerHour & false & "Request","Programable" \\
\cline { 2 - 5 } & WEATHERSTATION1_Temperature & TemperatureCelsius & false & "Request","Programable" \\
\hline
\end{tabular}

Table 6.5 Parameters of device Weather Station CEDINT

- To assess the performance of the authentication and authorization mechanisms developed two users have been created. One user is the Administrator (it has control 
on the whole system) and the second user is a non-administrator user. Each user has permission to access different spaces and parameter types.

- To verify the capability of the IoT platform to work with different communication protocols KNX and BATNet protocols have been used.

- To check the performance of the designed Web API in a real environment and to demonstrate the interoperability with existing applications (3D Virtual Reality and WeChat) REST client, a web-based user interface, 3D model graphical interface and we-chat application have been used.

- Customized user interfaces to control IoT devices have been built in the MiniGreenHouse.

The test bed is shown in Fig. 6.18.

All terms, used in this test bed are part of the vocabulary of BATOnt, the ontology developed in this thesis (BATOnt). BATOnt provides the semantic description needed in the system.

Summarizing, the test bed consists of:

- Two spaces:

- Space CEDINT, with SpaceType "Showroom".

- Space MiniGreenhouse, with SpaceType "Greenhouse"

- Two users:

- Administrator:

* Username: Johnny

* Password: 123456

- Non-administrator:

* username: GreenlabAdmin (this user is the greenhouse operator)

* Password: 123456

* Assigned role: GreenlabAdmin

- One role "GreenlabAdmin" is assigned with:

- Write permission to the space "MiniGreenhouse"

- Write permission to the parameter type "DimmingActuatorPercentage" 


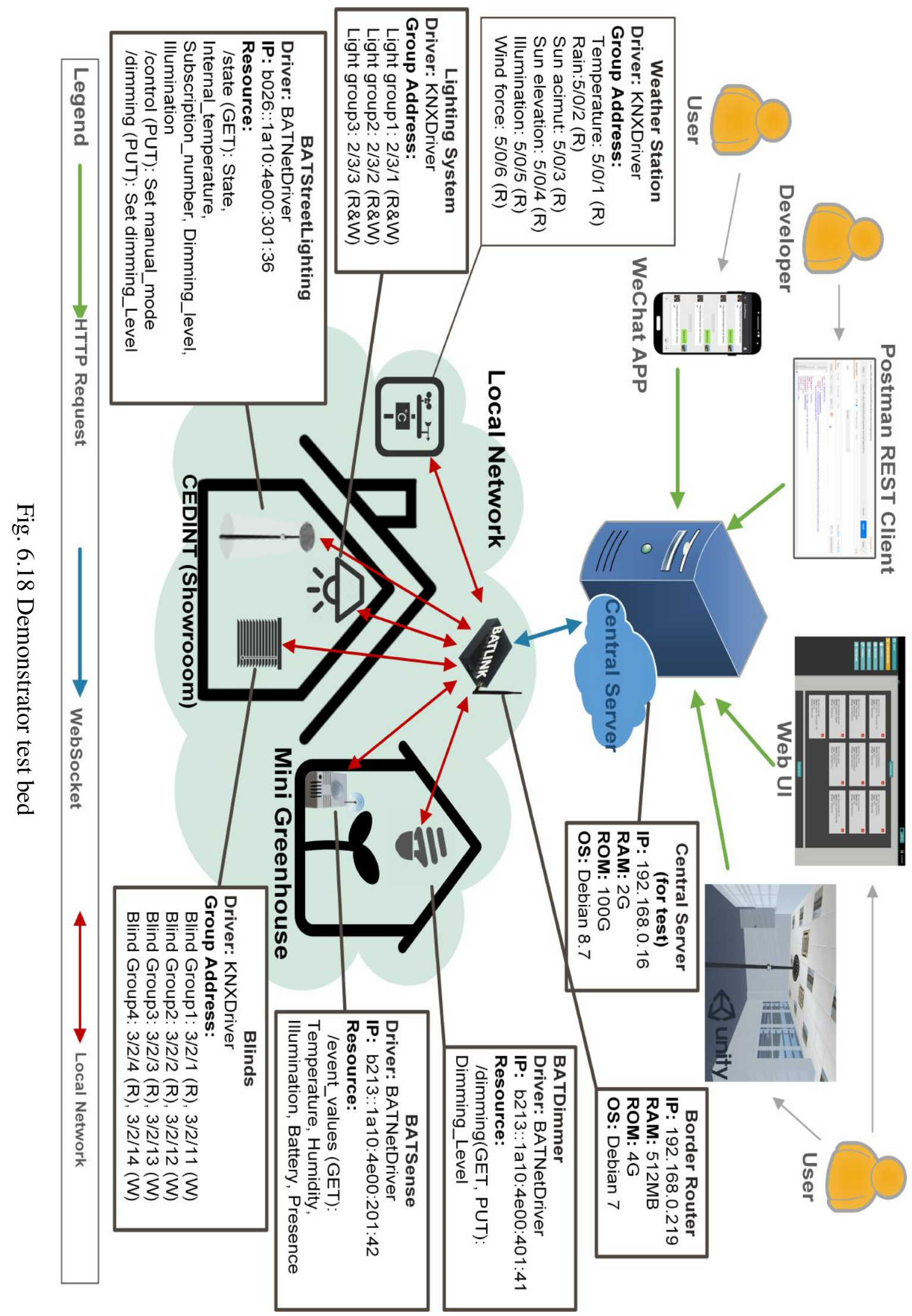


- Read permission to the parameter types "TemperatureCelsius", "Humidity", "IlluminationLevel" and "Presence".

On the basis of the defined fact rules and policy rules, the non-administrator GreenlabAdmin should only has the following permissions:

- Read permission to parameter BSN01-0042_Temperature

- Read permission to parameter BSN01-0042_Humidity

- Read permission to parameter BSN01-0042_Presence

- Read permission to parameter BSN01-0042_Illumination

- Write permission to parameter BDM01-0041_Dimmer

As a matter of fact, the generate PKG for GreenlabAdmin should only has the spaces, devices, users that are related with the above parameters.

\subsubsection{Test of Web API with REST Client}

To test the Web API implemented in the platform, the Postman-REST Client is used, which is a Chrome Extension provided by Google. As introduced in the previous chapters, a login request is needed to perform any subsequent requests. Fig. 6.19 shows the page of Postman when an unauthorized access is done. The request is denied and the HTTP status code 401 (Access unauthorized) is returned.

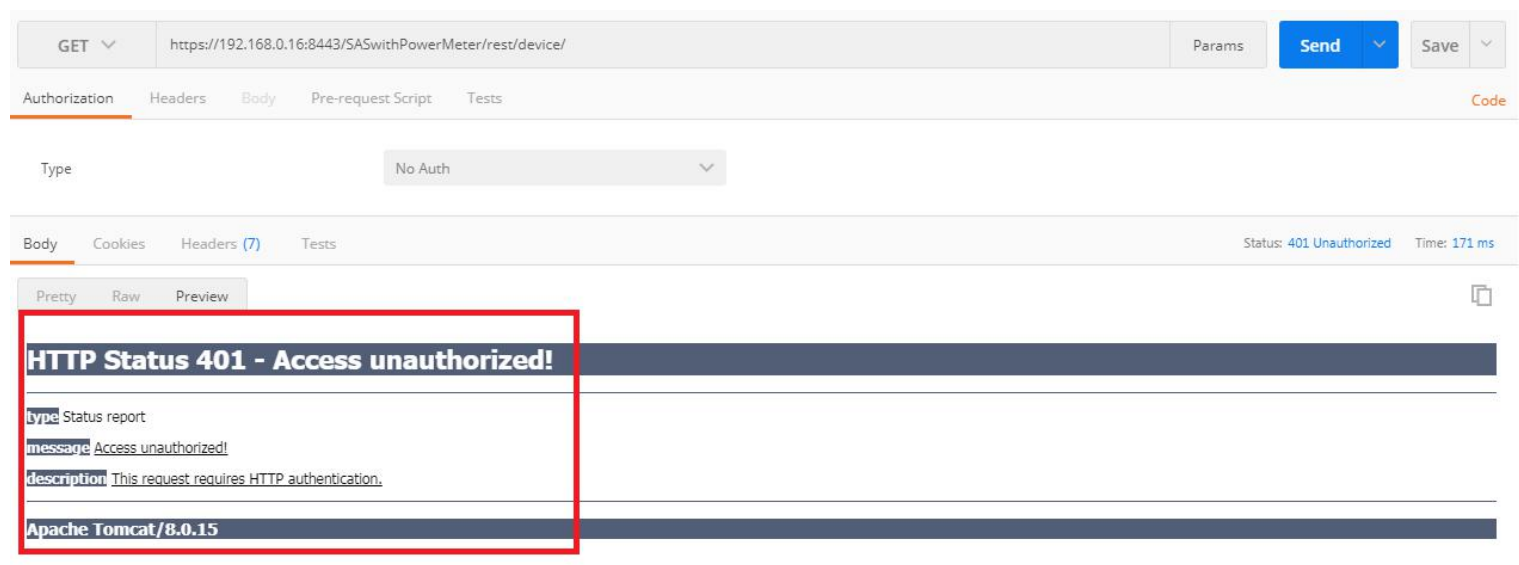

Fig. 6.19 Postman page when unauthorized access happens.

To communicate with the platform a user needs a valid username and password. If the login action successes, a valid token will be returned in the response, which should be put in 
the Authorization header of all the subsequent requests. This token will be used to retrieve the corresponding user PKG, ensuring that only the authorized resources can be accessed. The following sections will present the designed authorization mechanism by comparing the responses to the same request with different tokens. In one case the token belongs to the Administrator (Johnny) and the other case the token belongs to the non-administrator user (GreenlabAdmin).

\section{Perform login to get valid tokens}

Fig. 6.20 shows the Postman page for sending a login request with username "Johnny" and password "123456". A message is return in the form of JSON object, which indicates that the action has been executed successfully. The returned token is "eyJhbGciOiJIUzI1NiJ9.eyJpYXQ iOjE1MDQxOTY4ODcsInN1YiI6IlUwMDAwMDAwMDBCRTIEMzM 4IiwiaXNzIjoiYXV0aGVudGljYXRIIn0.-uSiL_zFc1t5vRinexiKEOqZN7ahifraFF8_cB2eGt8".

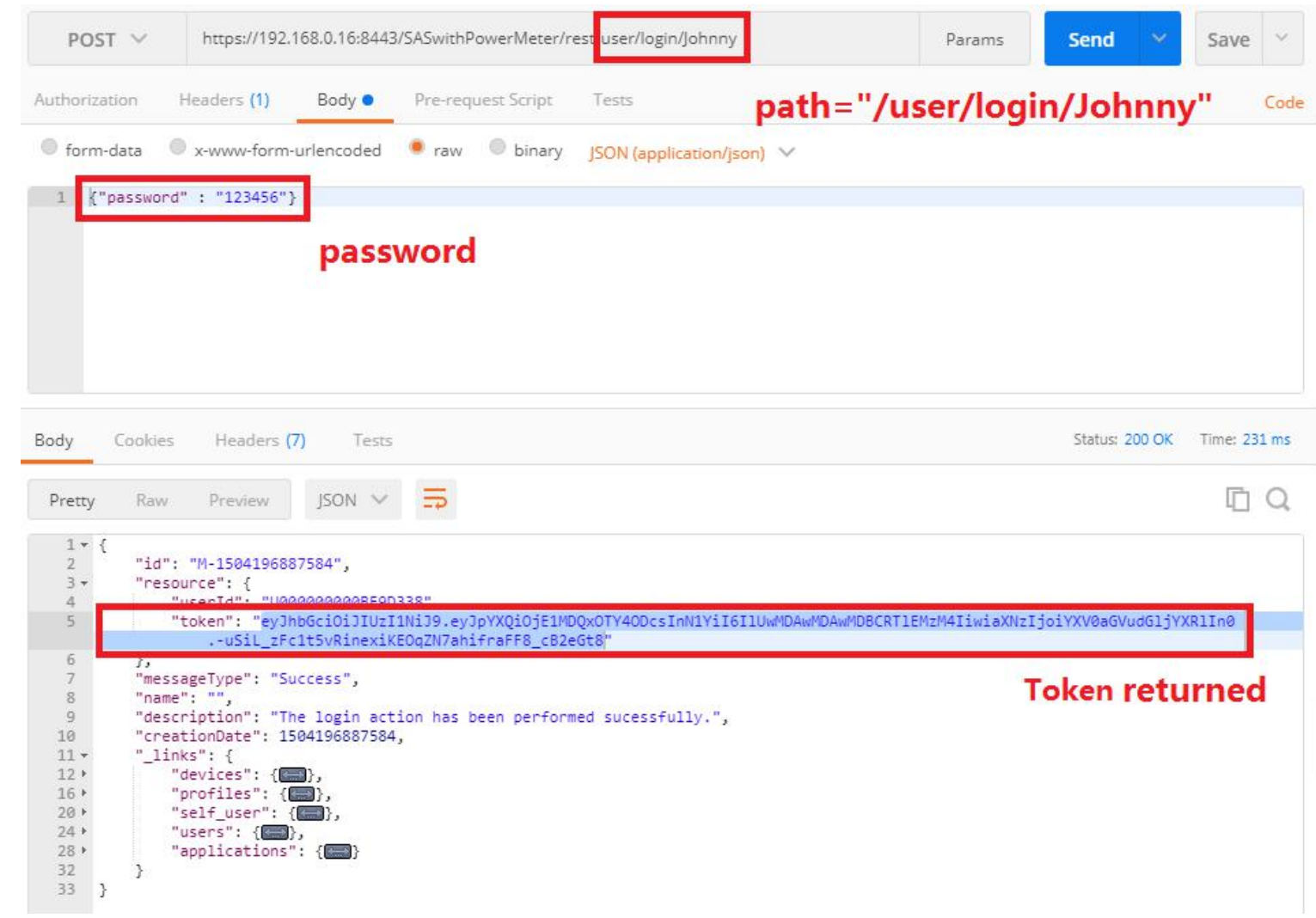

Fig. 6.20 Postman response to request login action for Johnny

Fig. 6.21 shows the returned message of the login action with username "GreenlabAdmin", including the generated token "eyJhbGciOiJIUzI1NiJ9.eyJpYXQiOjE1MDQyNDgzMTgsInN 
1YiI6IlUwMDAwMDAwMEJFQURENjQyIiwiaXNzIjoiYXV0aGVudGljYXRIIn0.GFfiaKG _wHtzYz iuYj3aqS3sGrBH0J-y_iDX745nhB8".

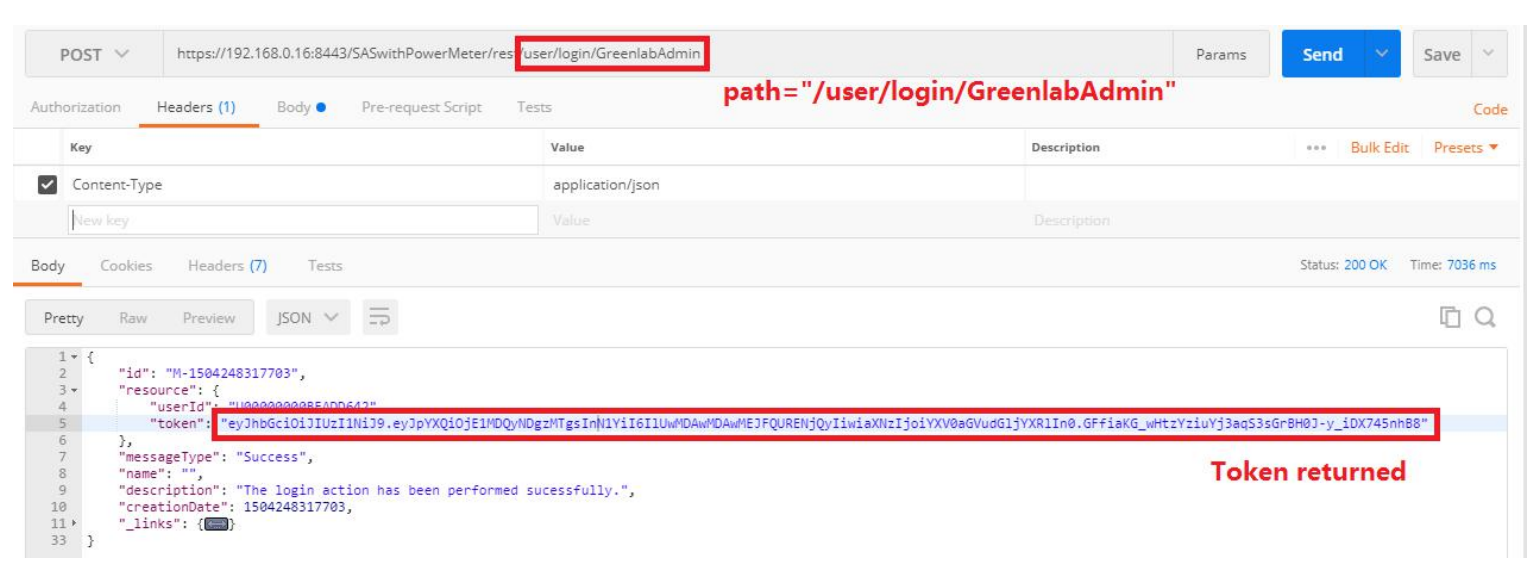

Fig. 6.21 Send request by Postman to perform login action for GreenlabAdmin

After a successful login action, the corresponding PKGs are generated for Johnny and GreenlabAdmin.

Tokens are included in the Authorization header in all requests. Each token corresponds to a PKG that contains the information that the user can access. The response varies depending on the user, because each user has its own PKGs.

\section{Get users}

A GET request with path "/user" returns the list of all users created. Fig. 6.22 shows the list of users for Johnny. Fig. 6.23 shows the list of users for user GreenlabAdmin. 


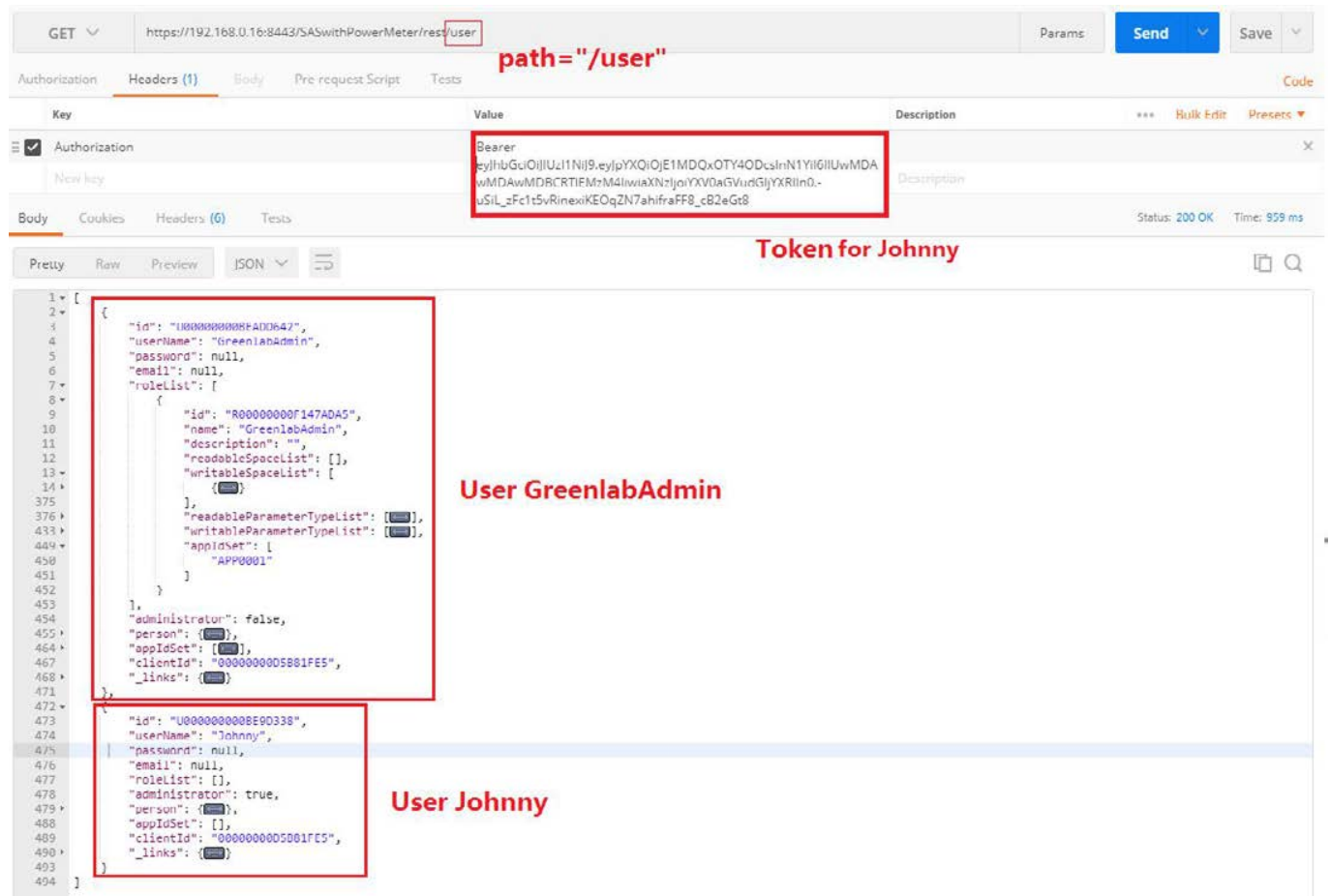

Fig. 6.22 Postman response to request get all user for Johnny

As shown in Fig. 6.22, the list of users for Johnny contains two users: Johnny and GreenlabAdmin, 


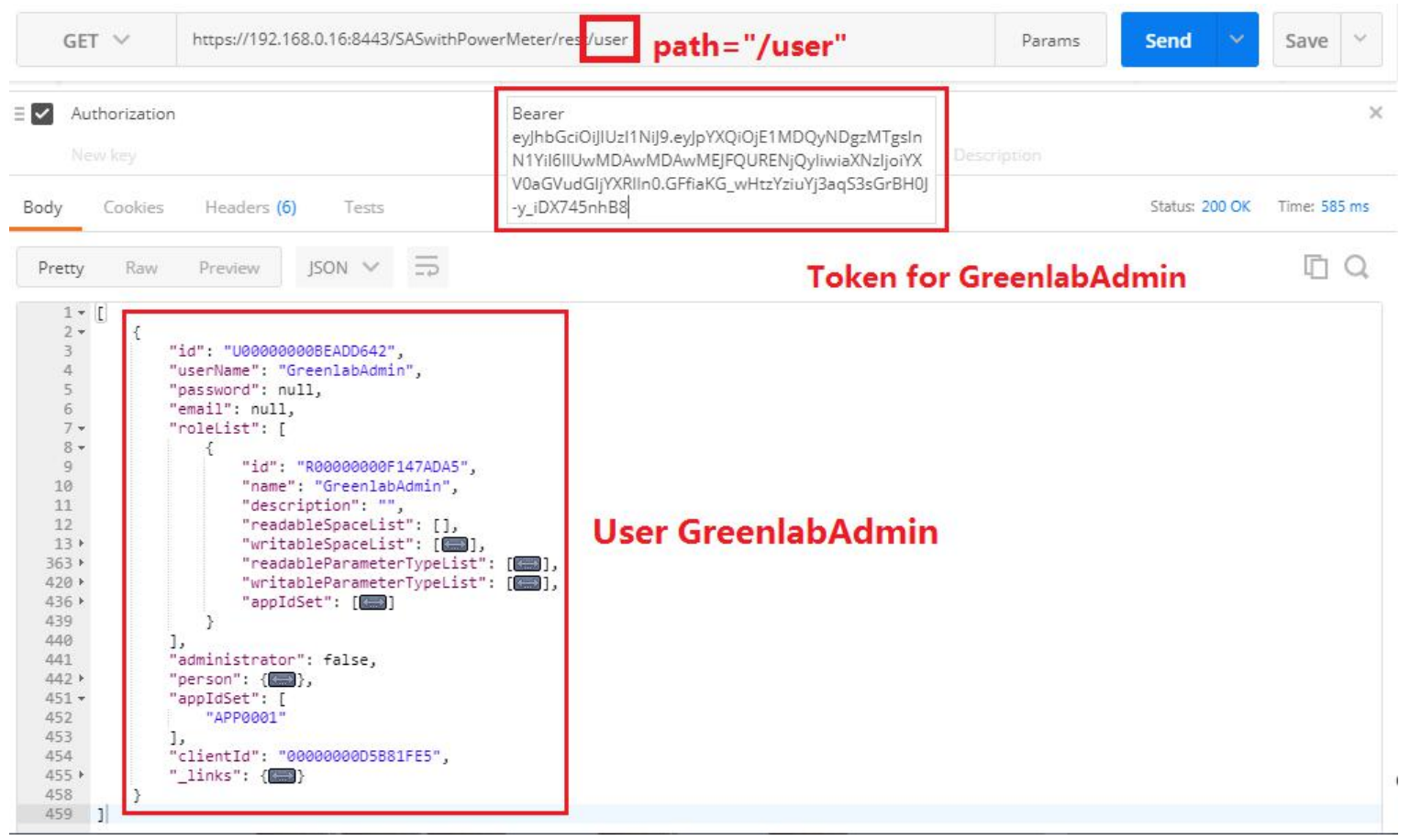

Fig. 6.23 Postman response to request get all user for user GreenlabAdmin

As shown in Fig. 6.23 the list of users for GreenlabAdmin is itself.

\section{Get spaces}

A GET request with path "/space" returns all the spaces that a user is authorized to access. Fig. 6.24 shows the list of spaces for Johnny.

Fig. 6.24 shows the list of spaces for Johnny. 


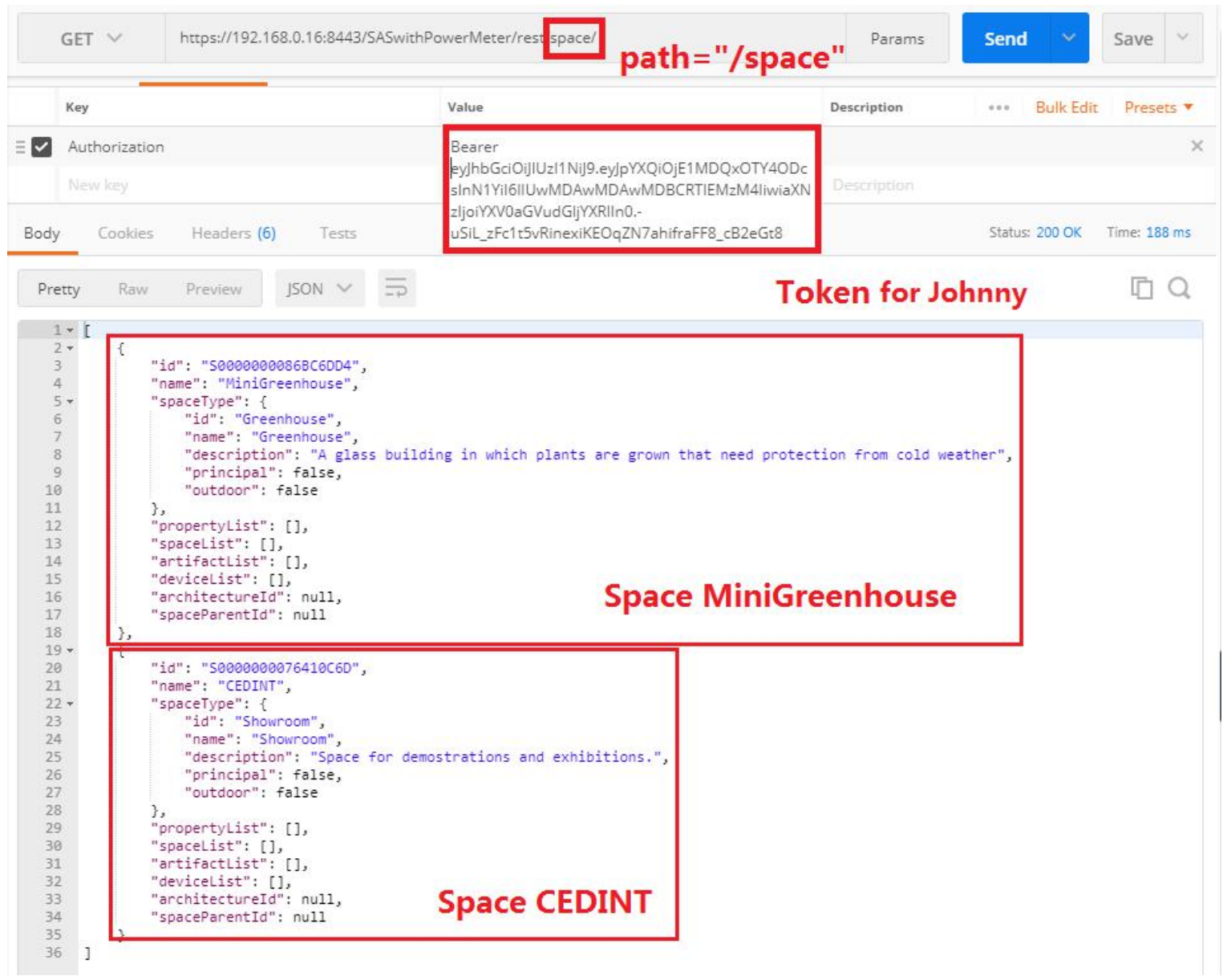

Fig. 6.24 Postman response to request get spaces for Johnny

Fig. 6.25 shows the list of spaces for user GreenlabAdmin.

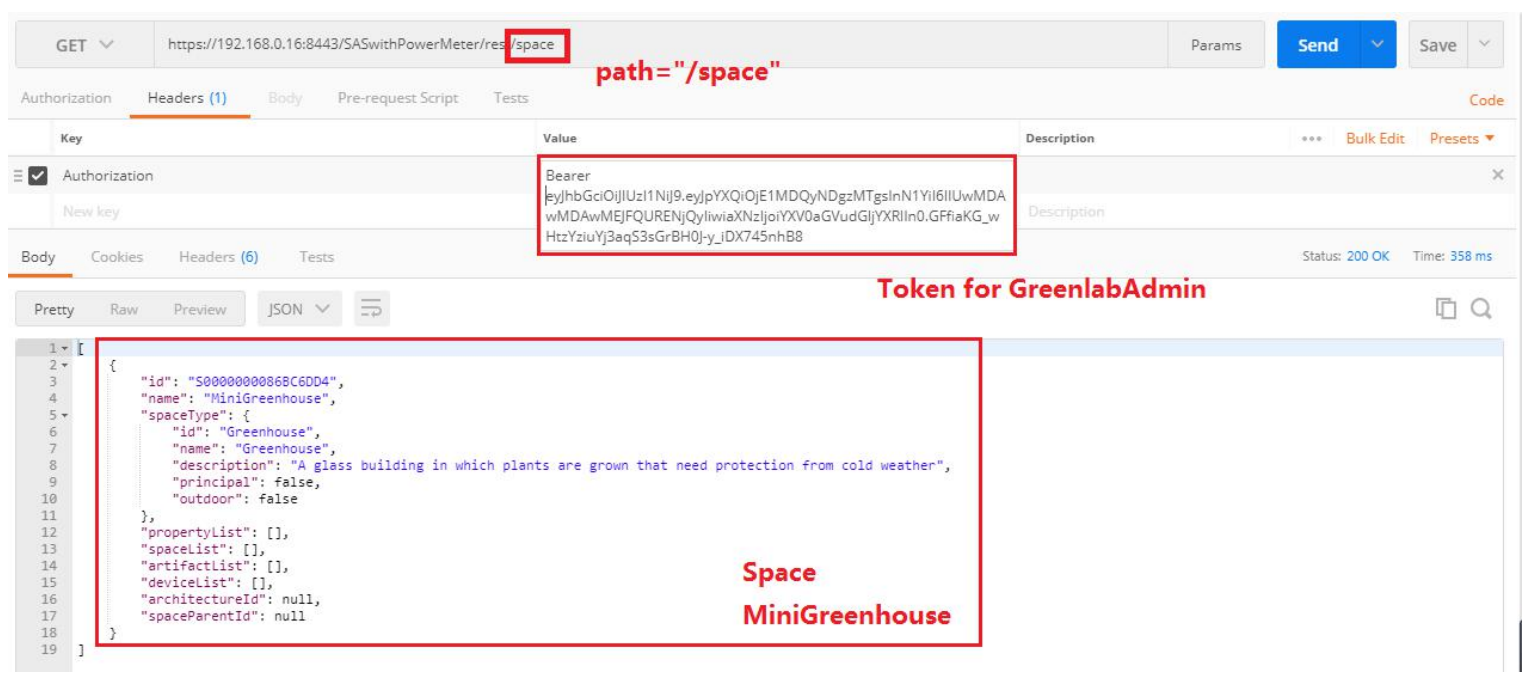

Fig. 6.25 Send request by Postman to get all the spaces for non-administrator user 
Comparing Fig. 6.24 and Fig. 6.25, Johnny has access to both spaces (CEDINT and MiniGreenhouse), while GreenlabAdmin only has access to MiniGreenhouse space.

\section{Get devices}

A GET request with path "/ device" returns all the devices that a user is authorized to access.

Fig. 6.26 shows the list of devices for Johnny.

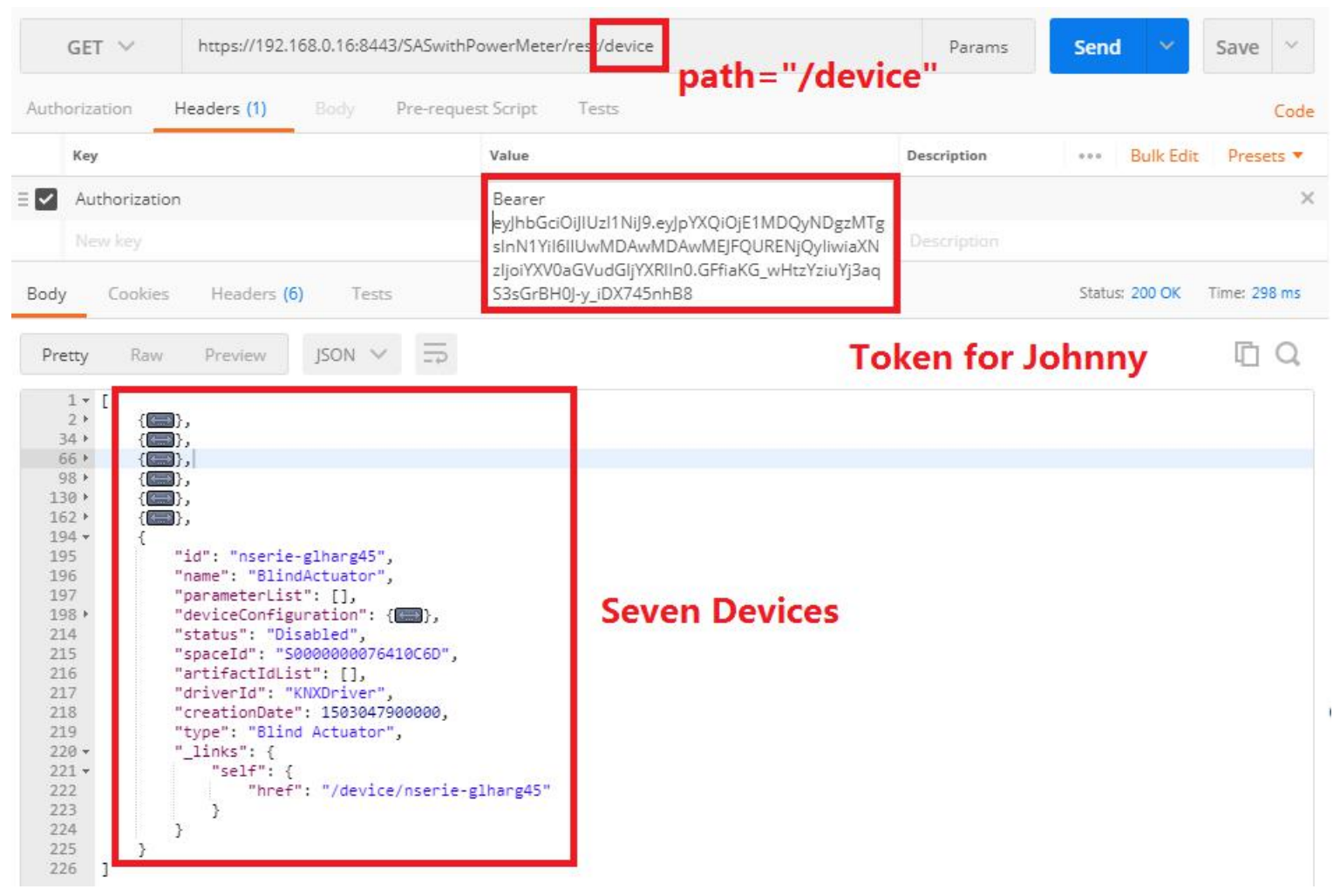

Fig. 6.26 Postman response to request get all the devices for Johnny

Each object in the returned JSON array contains the basic information of each device. Fig. 6.26 shows the JSON representation of the device BlindActuator which contains id, name, configuration information, status, driver and type. 


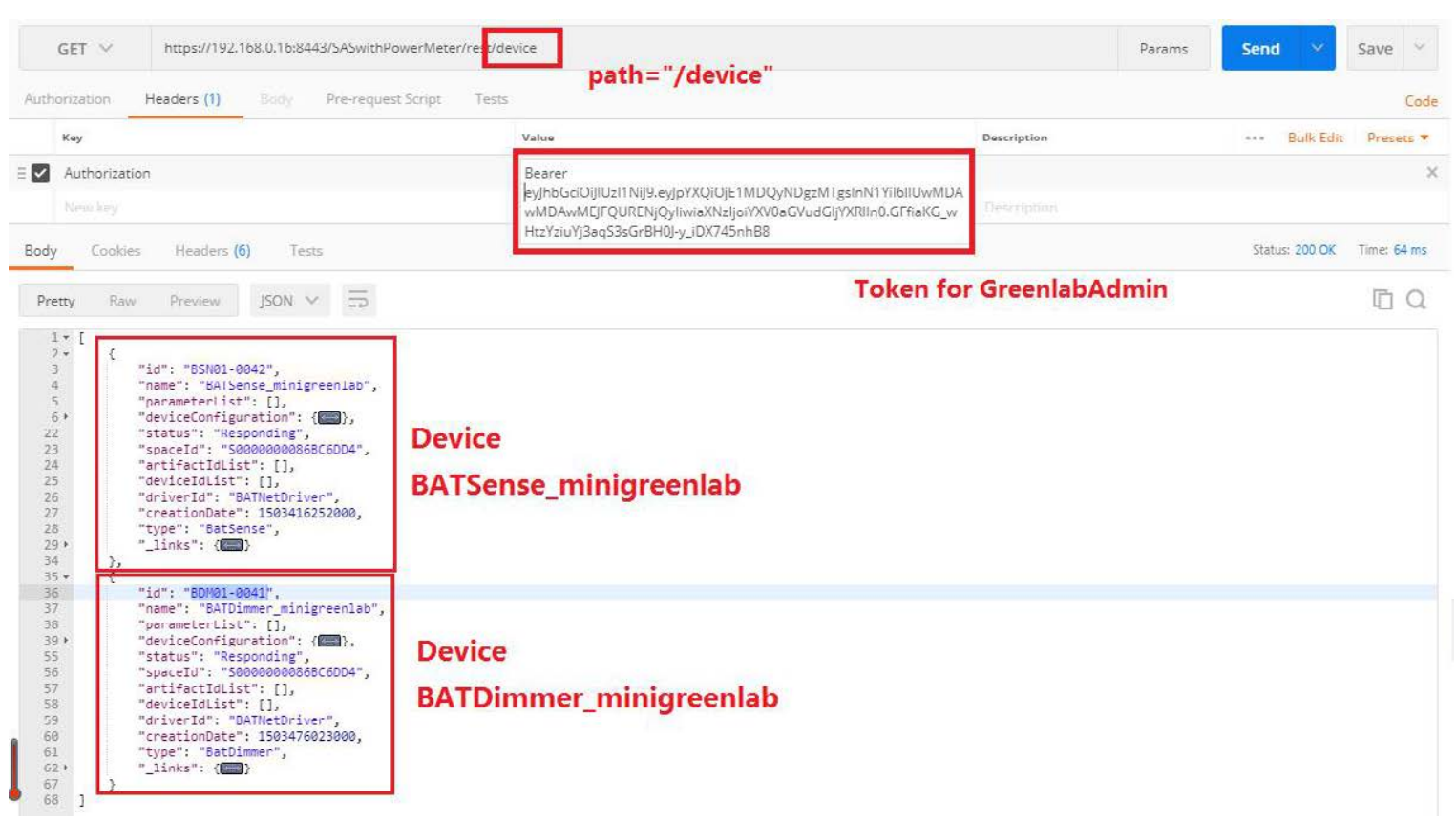

Fig. 6.27 Postman response to request get all the devices for GreenlabAdmin

Fig. 6.27 shows the list of devices for GreenlabAdmin.

As illustrated in Fig. 6.26 and Fig. 6.27, there are seven devices in the returned list for Johnny, and two devices for user GreenlabAdmin.

Fig. 6.26 shows the value of the key "_links". It indicates a list of links that can be followed to get related resources. The "self" link represents the URI of the main resource. By following this link, a GET request can be issued furthermore to retrieve the details of this device, while in the returned JSON object, all the belonging parameters of the devices are illustrated, as shown in Fig. 6.28, 


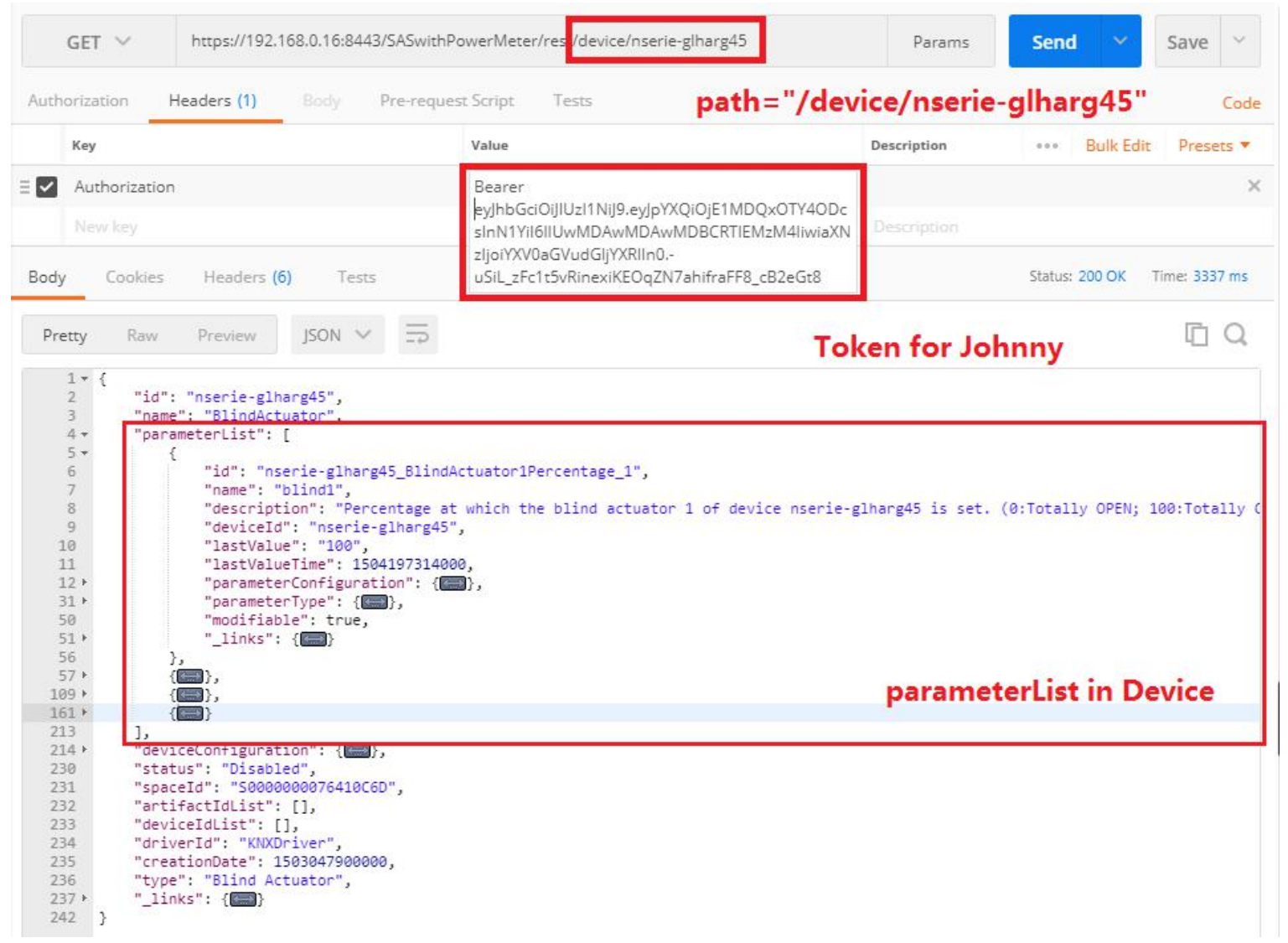

Fig. 6.28 Postman response to request get a specified device

\section{Get the parameters of a device}

On the basis of the designed authorization mechanism, users can only access the parameters that are contained in their own PKGs. Fig. 6.29 and Fig. 6.30 shows respectively the representation of the device resource for Johnny and user GreenlabAdmin. As shown in Fig. 6.29, the parameterList for Johnny has five parameters, while Fig. 6.30 shows that for user GreenlabAdmin the parameterList has only four parameters. Since the parameter BSN01-0042_Battery has parameter type BatteryVoltage and user GreenlabAdmin has not been granted any permission to this parameter type, this parameter is not contained in the PKG for user GreenlabAdmin. 


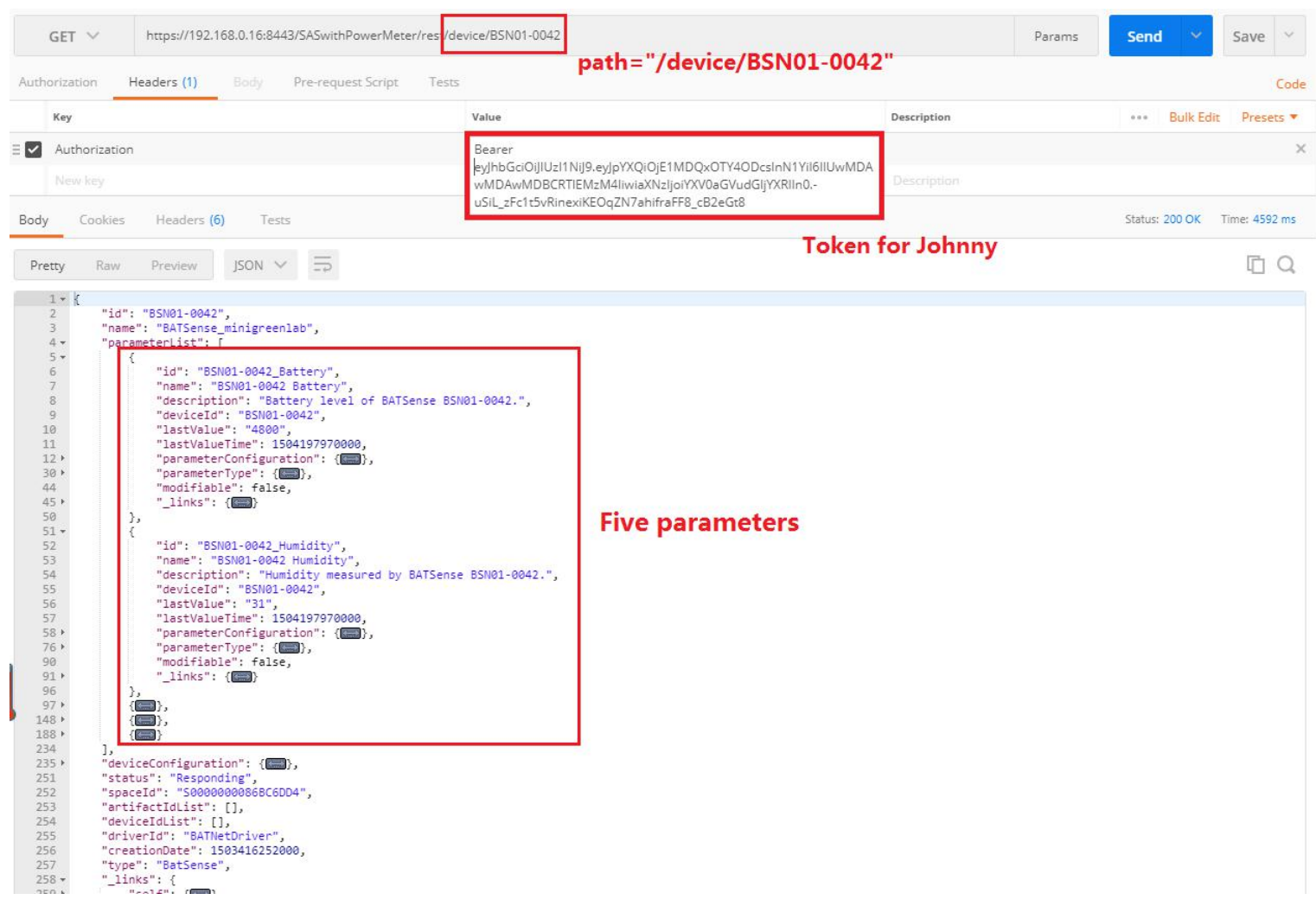

Fig. 6.29 Postman response to request get device BSN01-0042 for Johnny

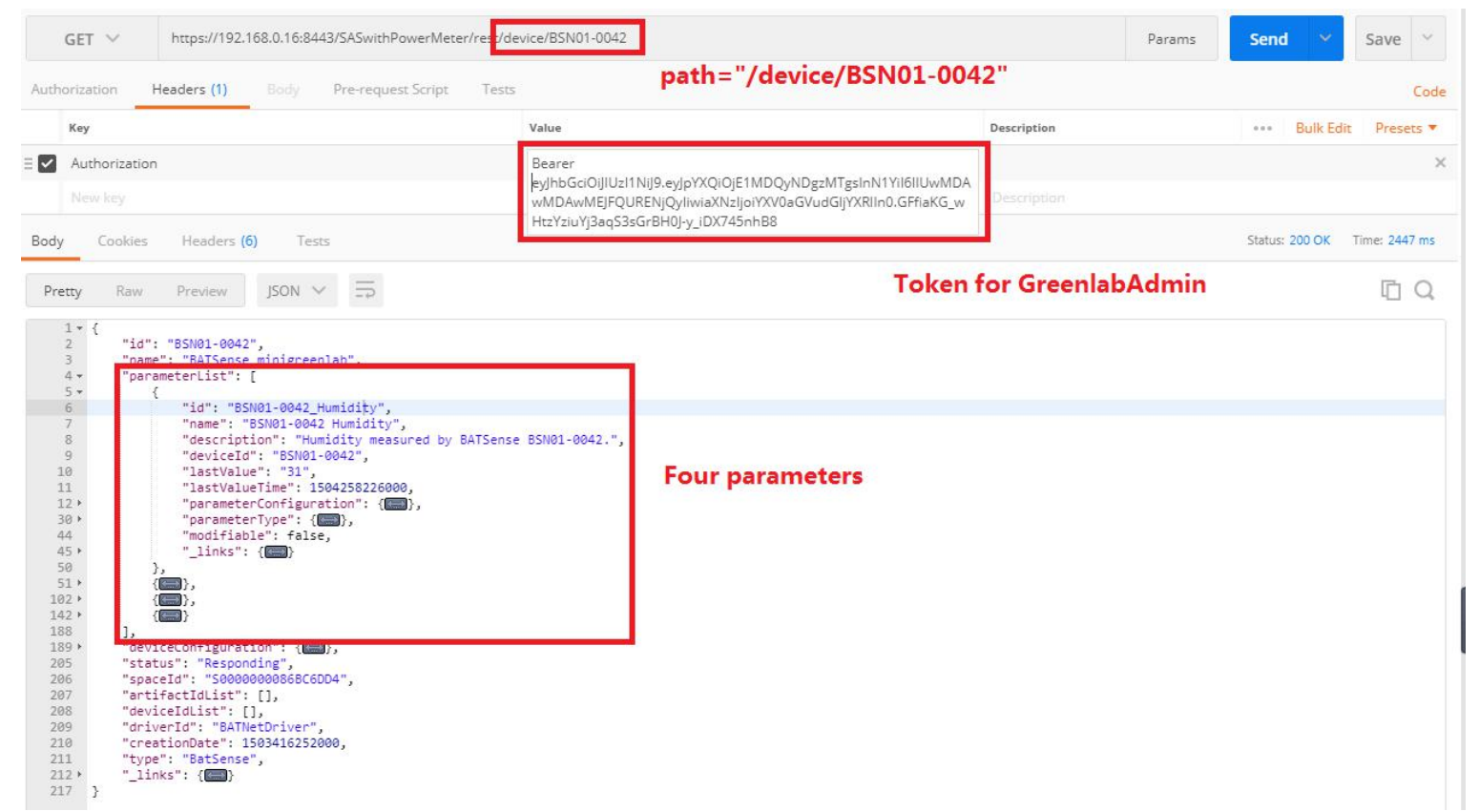

Fig. 6.30 Postman response to request get device BSN01-0042 for GreenlabAdmin 


\section{Get parameters}

A GET request with path "/parameter" returns all the parameters that a user is authorized to access. Fig. 6.31 shows the list of parameters for Johnny.

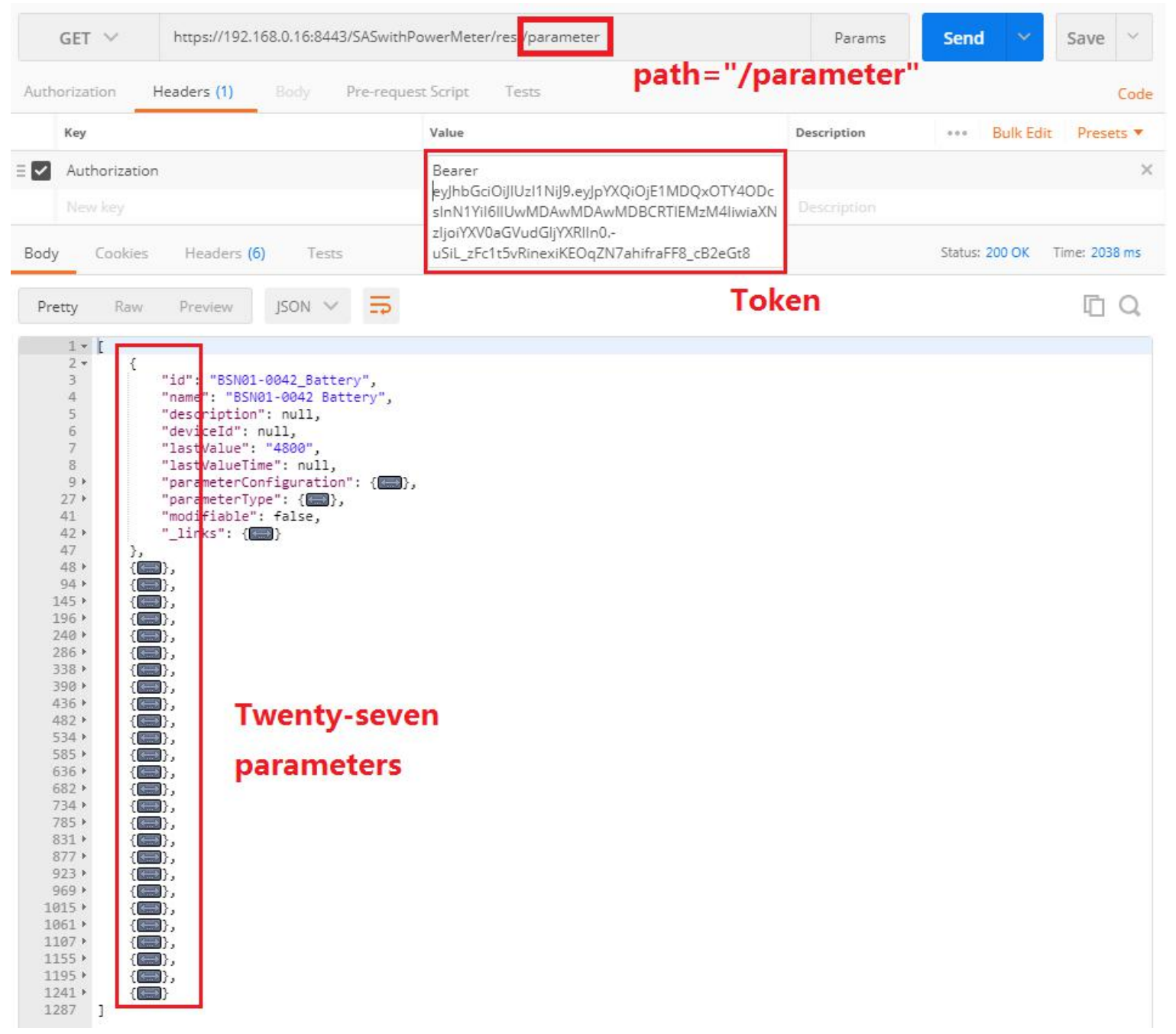

Fig. 6.31 Postman response to request get all the parameters for Johnny

Fig. 6.32 shows the list of parameters for GreenlabAdmin. 


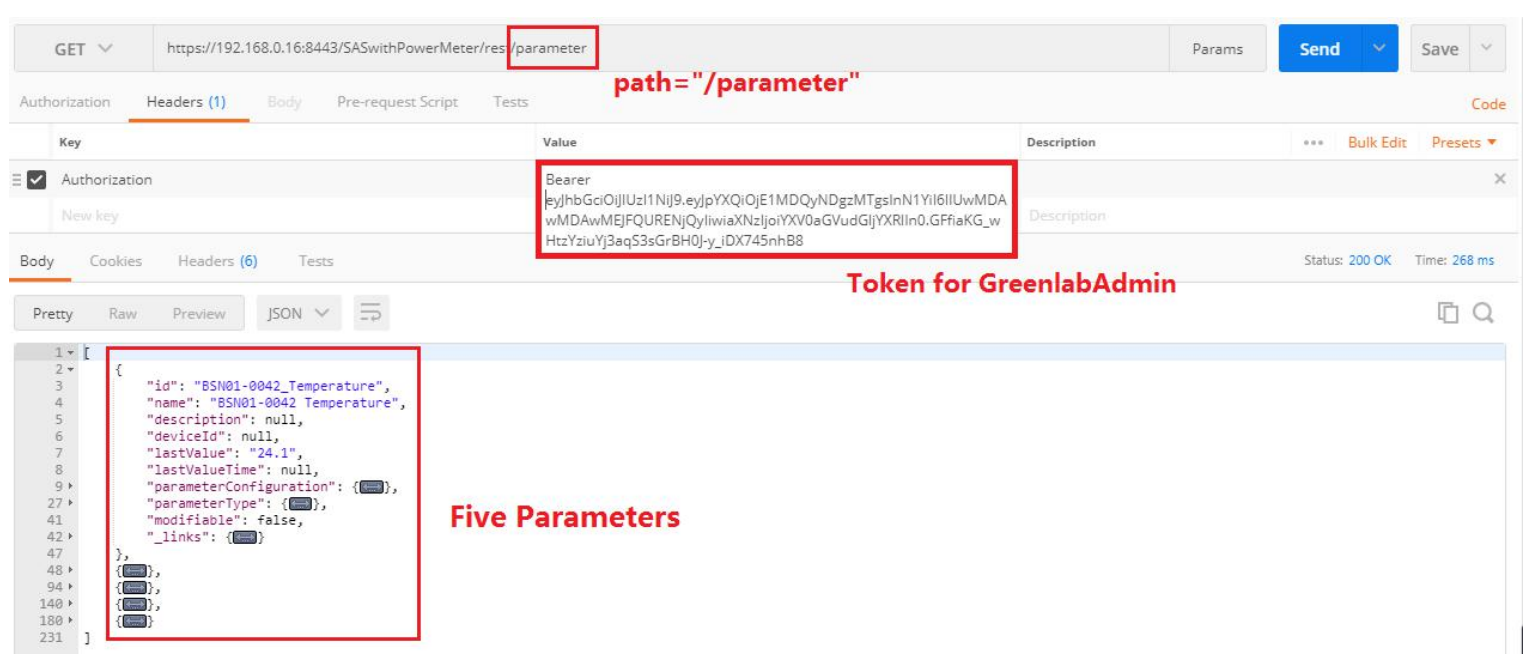

Fig. 6.32 Postman response to request get all the parameters for GreenlabAdmin

As illustrated in Fig. 6.31 and Fig. 6.32, there are twenty-seven parameters in the returned list for Johnny, and five parameters for user GreenlabAdmin.

\section{Update a parameter}

The control of devices is carried out by changing the value of the corresponding parameter. To change the value of a parameter, a PUT request is sent with path "/parameter/local/userUpdate/ $\left\{\mathrm{ID}_{\text {parameter }}\right\}$ ", where $\mathrm{ID}_{\text {parameter }}$ indicates the ID of the target parameter. Fig. 6.33 shows how a user modifies the value of an authorized parameter. A message is returned indicating the result of the operation. In this case, the operation has been executed successfully.

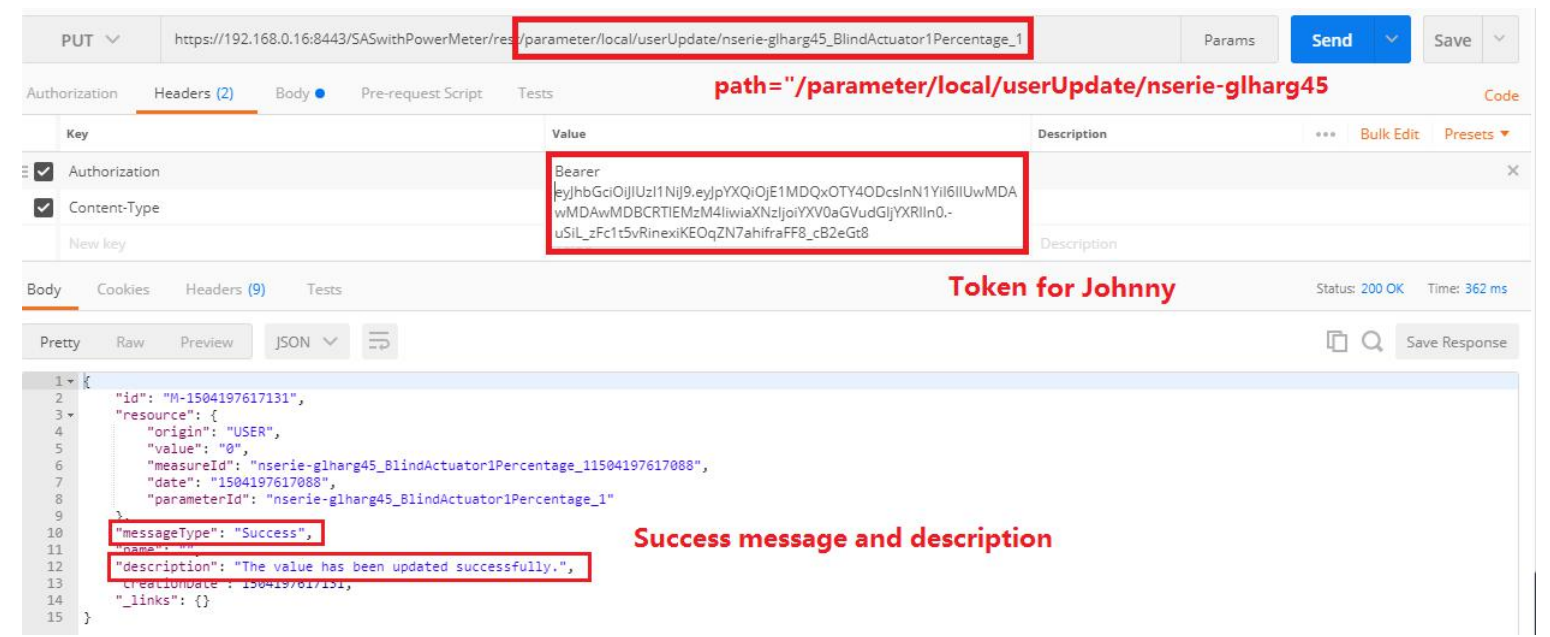

Fig. 6.33 Postman response to change the value of a parameter as an administrator 
The value of a parameter can be changed by a user only if the user has Write permission to the parameter, i.e. the id of the parameter is in the Writeable Parameter List (WPL), as explained in Chapter 5. Otherwise, the request will be denied and a HTTP status code 401 will be returned.

Fig. 6.34 shows the case that the user has only read permission to the target parameter.

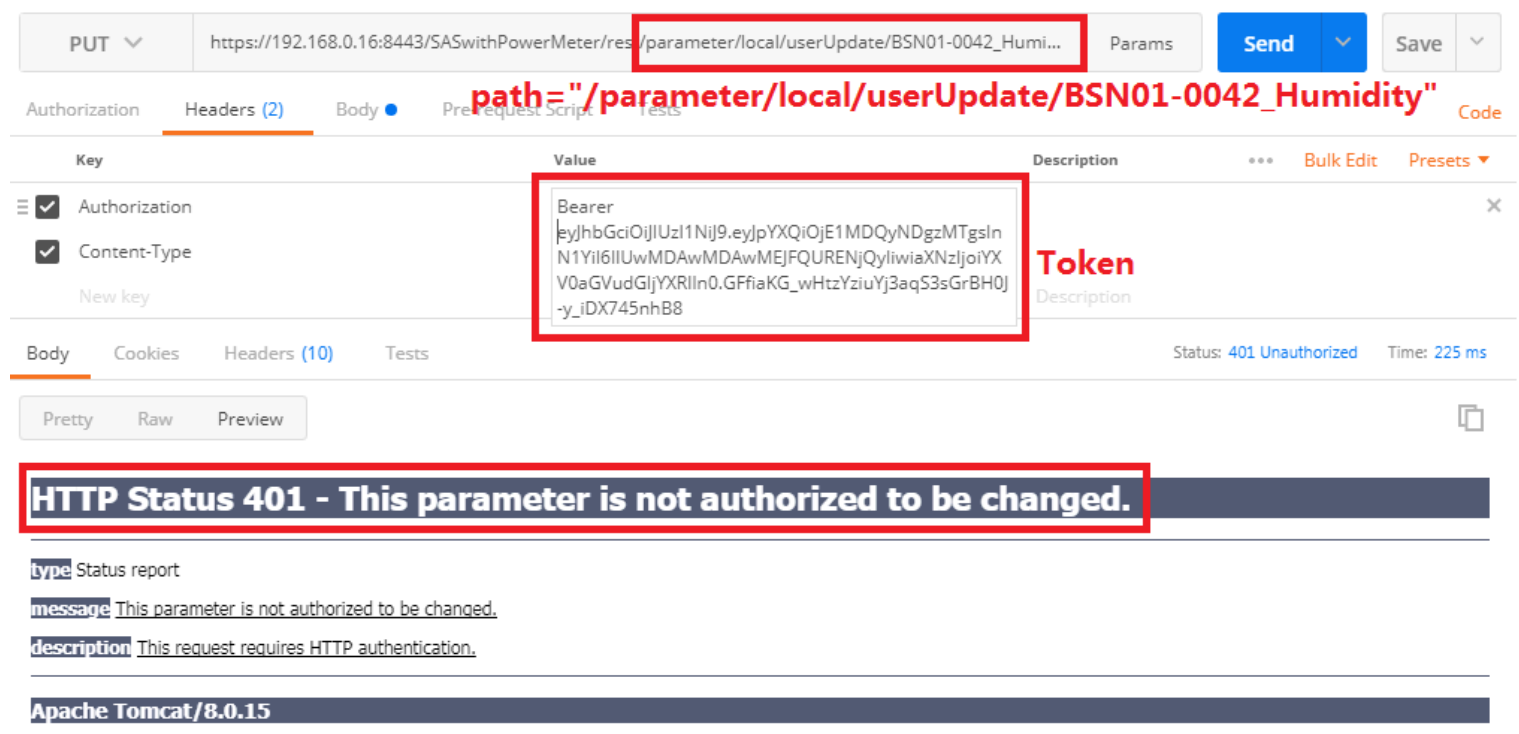

Fig. 6.34 Postman response to change the value of a parameter with Read permission

The REST Client terminal provides a convenient way for developers to debug and test the program. However, it is necessary to have a friendly interface for the communication with the platform. In the following section, a web-based user interface is presented.

\subsubsection{Web-based user interface}

The platform provides a friendly web-based user interface where the user can manage the configurations about users, spaces, devices and applications. In the following part, the user interface will be introduced from four aspects:

- Login as administrator

- Login as non-administrator user

- Query and control devices

- Control device through applications 


\section{Login as administrator}

After successfully logged in, the user is able to manage the configuration of users, devices, spaces, scenes and rules through the control panel. The administrator can create, modify, delete roles, users and assign different roles to each user. Through the control panel of role management shown in Fig. 6.35, a new role GreenlabAdmin has been created, which is assigned:

- With respect to space: Write permission to the space MiniGreenhouse

- With respect to parameter type:

- Read permission to Illumination Level, Presence, Humidity and Temperature Celsius

- Write permission to Dimming Actuator Level

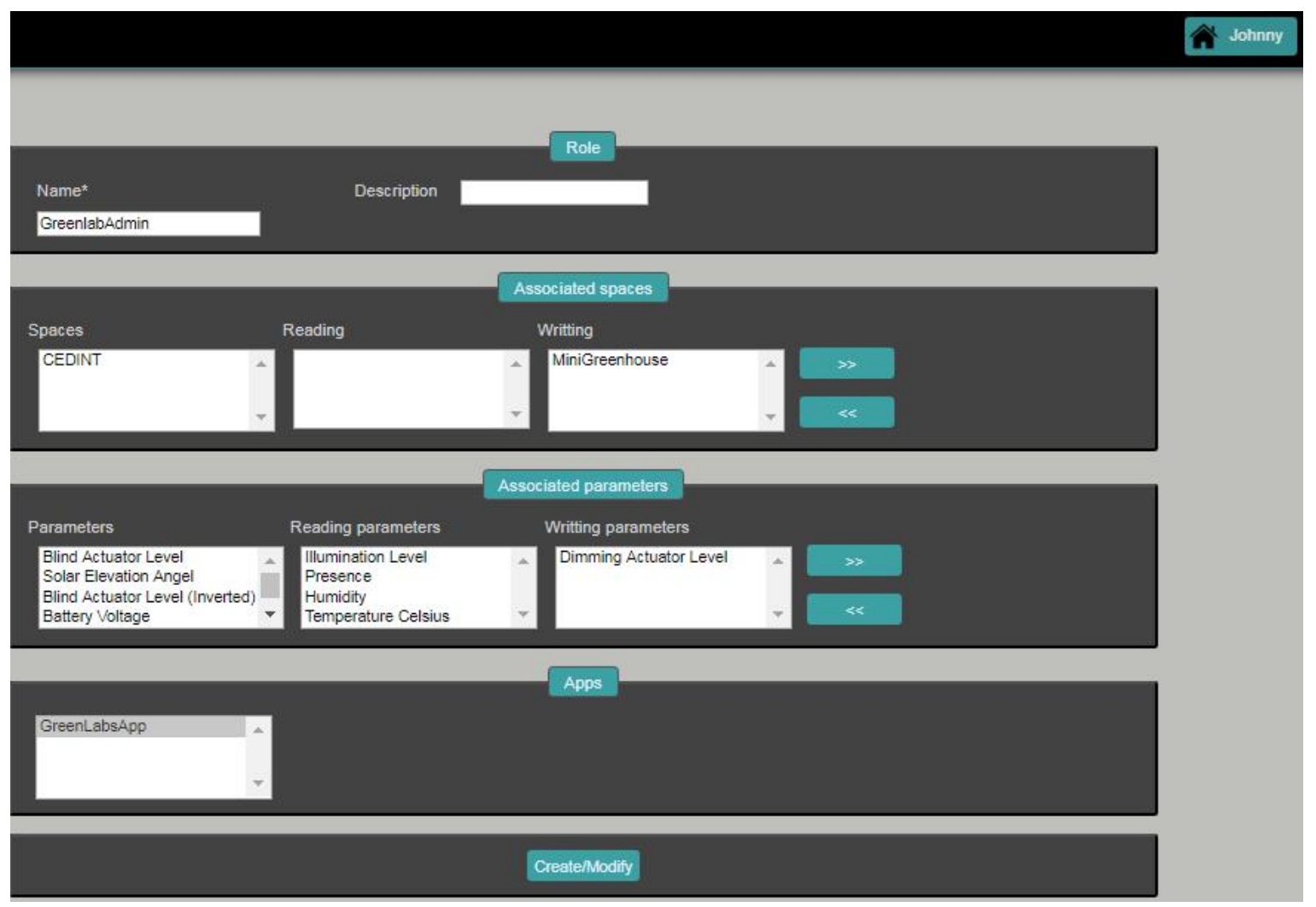

Fig. 6.35 Control panel of role management

Fig. 6.36 shows the control panel to create/modify users. Through this page, Johnny creates a new user GreenlabAdmin and assigns it with the role "GreenlabAdmin". 


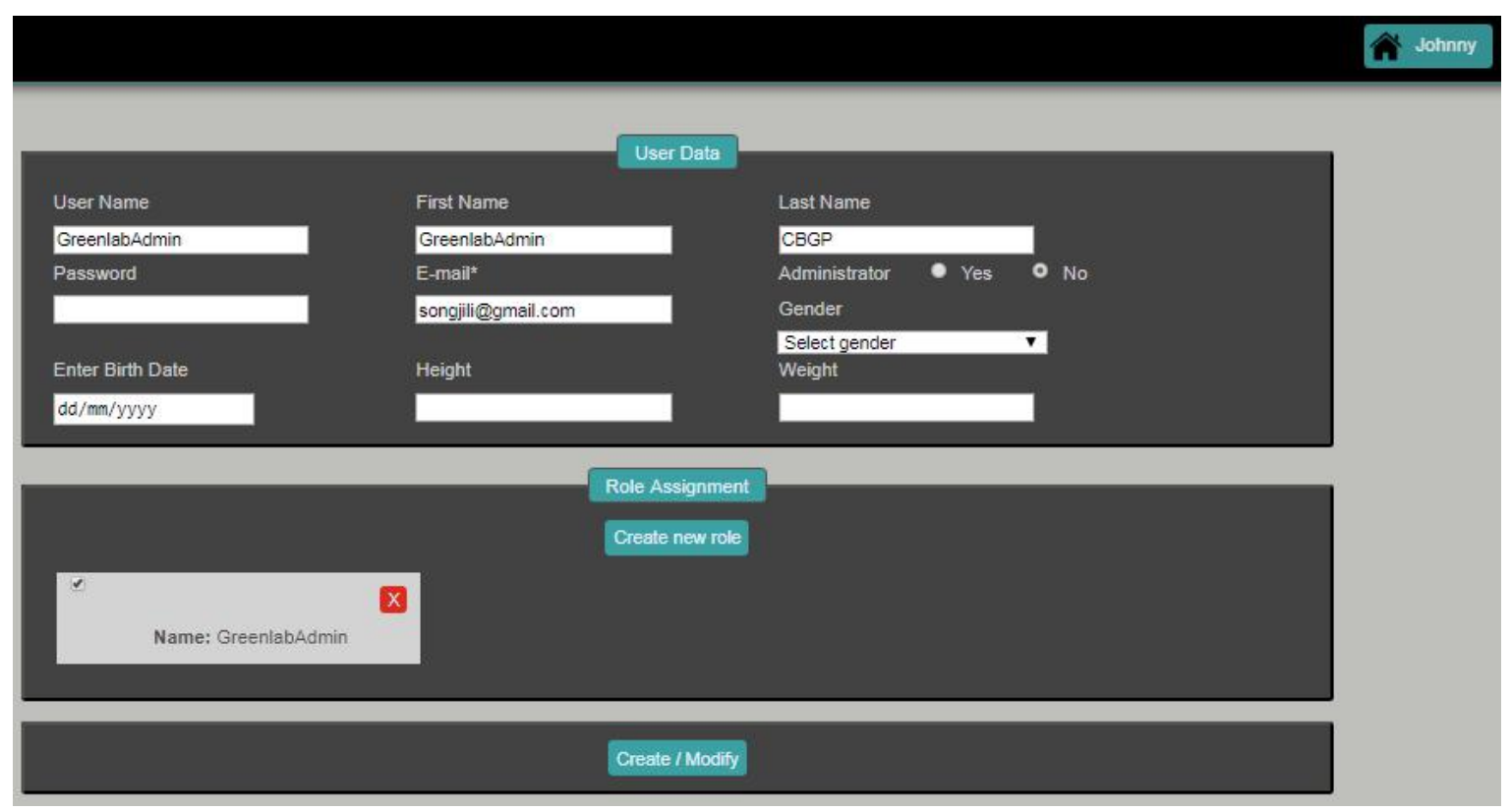

Fig. 6.36 Control panel of user creation/modification

After successfully creating the user GreenlabAdmin, the web is redirected to the control panel of user management for Johnny. As shown in Fig. 6.37, there are two users listed, Johnny and GreenlabAdmin, which both belong to the same client.

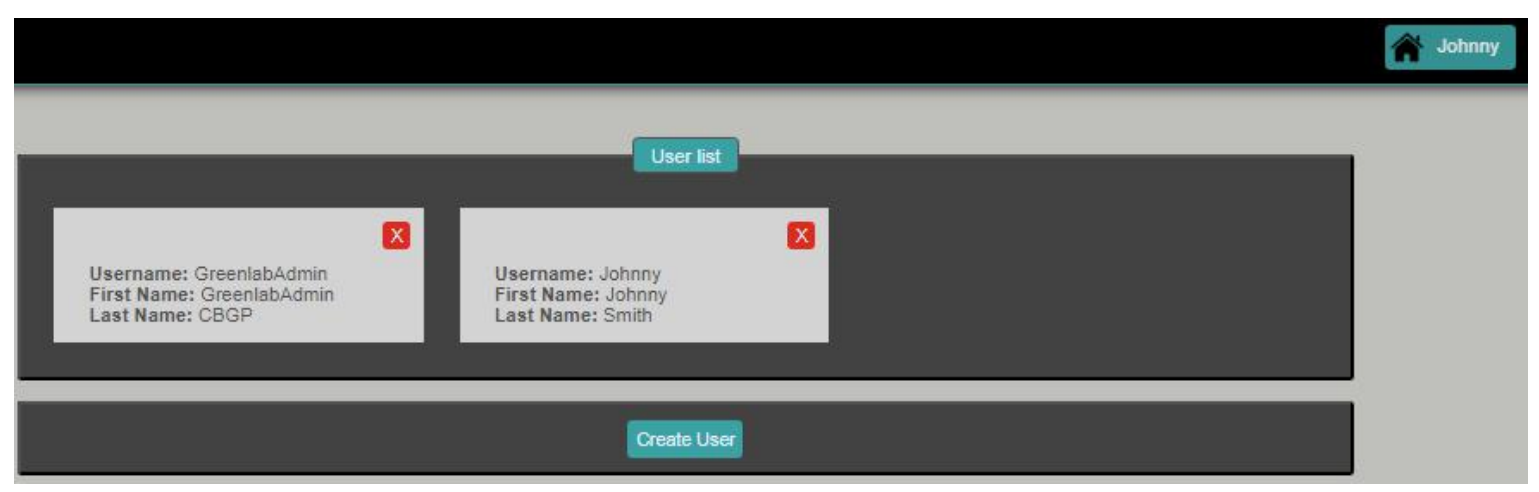

Fig. 6.37 Control panel of user management for Johnny

Fig. 6.38 shows the control panel of spaces management for Johnny. As mentioned before, Johnny is authorized to access two spaces, "CEDINT" and "MiniGreenhouse". 


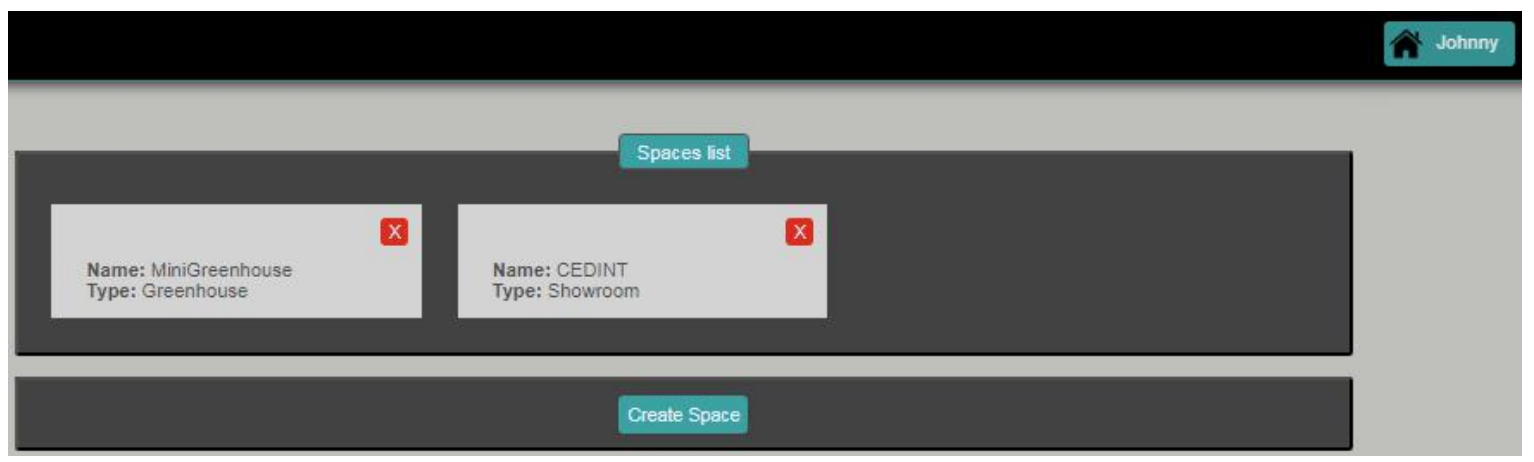

Fig. 6.38 Control panel of space management for Johnny

Fig. 6.39 illustrates the control panel of device management, where all the devices that Johnny is authorized to access, i.e. all the devices in the Table 6.2 are listed.

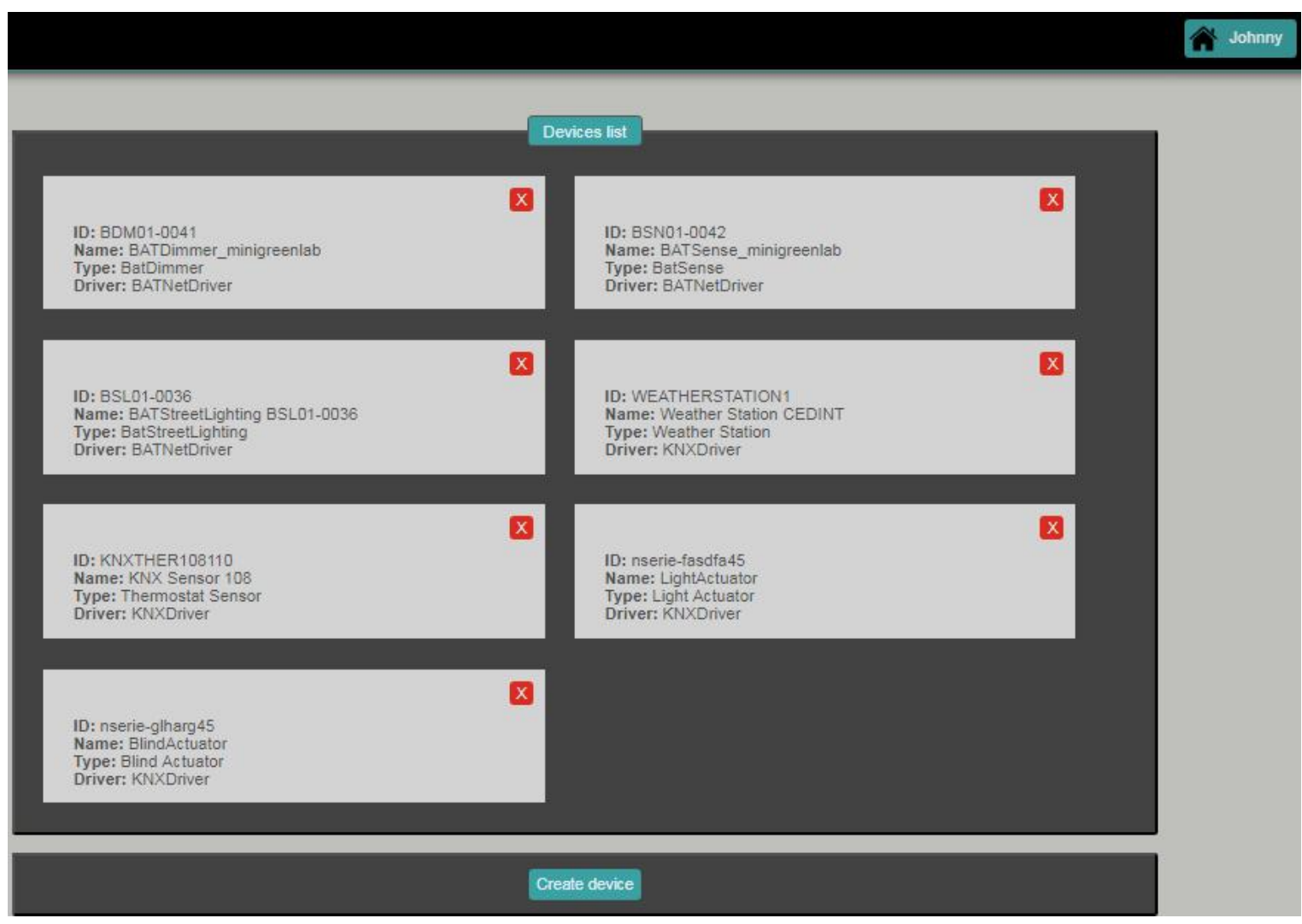

Fig. 6.39 Control panel of device management for Johnny

\section{Login as non-administrator user}

After having logged in as GreenlabAdmin, which is a non-administrator, the user can see and modify the information which belongs to himself. Fig. 6.40 shows the control panel of user 
management for GreenlabAdmin. There is only one user "GreenlabAdmin". In addition, the function of creating new users is disabled as an non-administrator is not allowed to create new users.

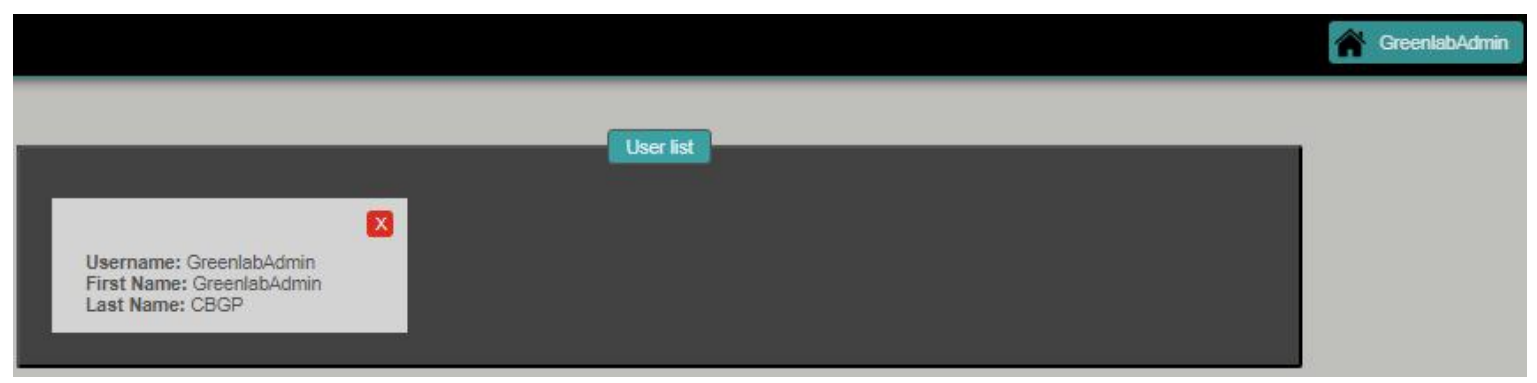

Fig. 6.40 Control panel of user management for GreenlabAdmin

Fig. 6.41 shows all the spaces that GreenlabAdmin is authorized to access, which is "Mini Greenhouse". Meanwhile, as a non-administrator user, GreenlabAdmin is not allowed to create and modify the space configuration.

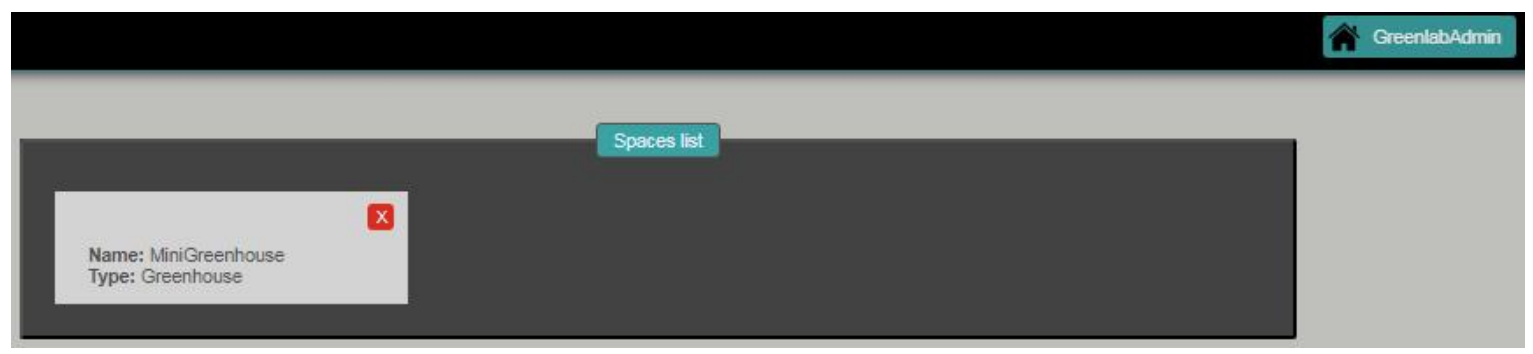

Fig. 6.41 Control panel of space management for GreenlabAdmin

Fig. 6.42 shows the control panel of device management for GreenlabAdmin, where only the two authorized devices are listed: BATSense_minigreenlab and BATDimmer_minigreenlab.

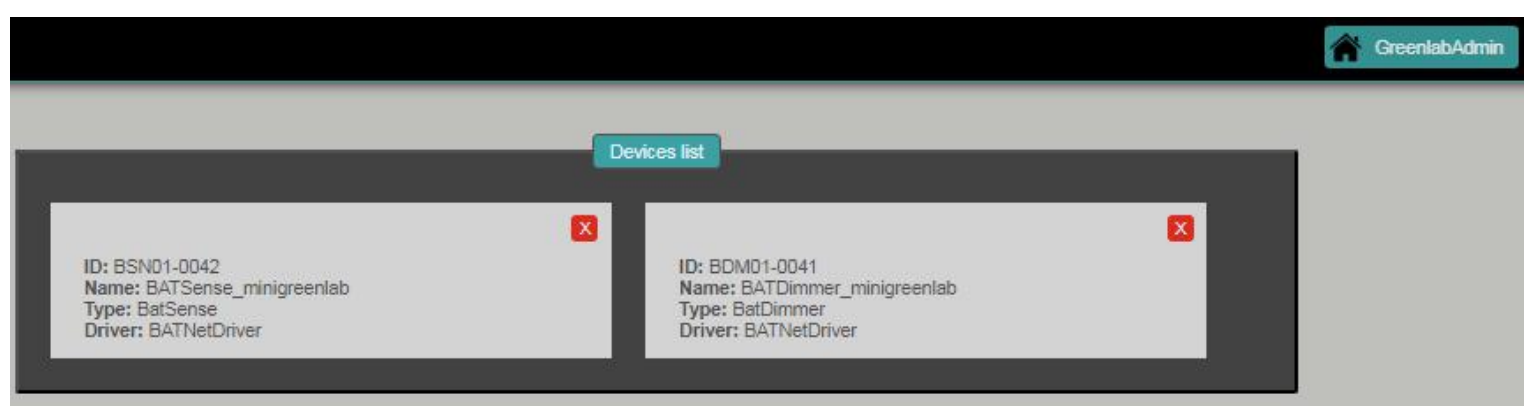

Fig. 6.42 Control panel of device management for GreenlabAdmin

After clicking the device BATSense_minigreenlab, the user is redirected to the control panel of device configuration for BATSense, as shown in Fig. 6.43. Since GreenlabAdmin is 
not assigned any permission to the ParameterType "Battery Voltage", the parameter "BSN010042_Battery" is not shown in its control panel.

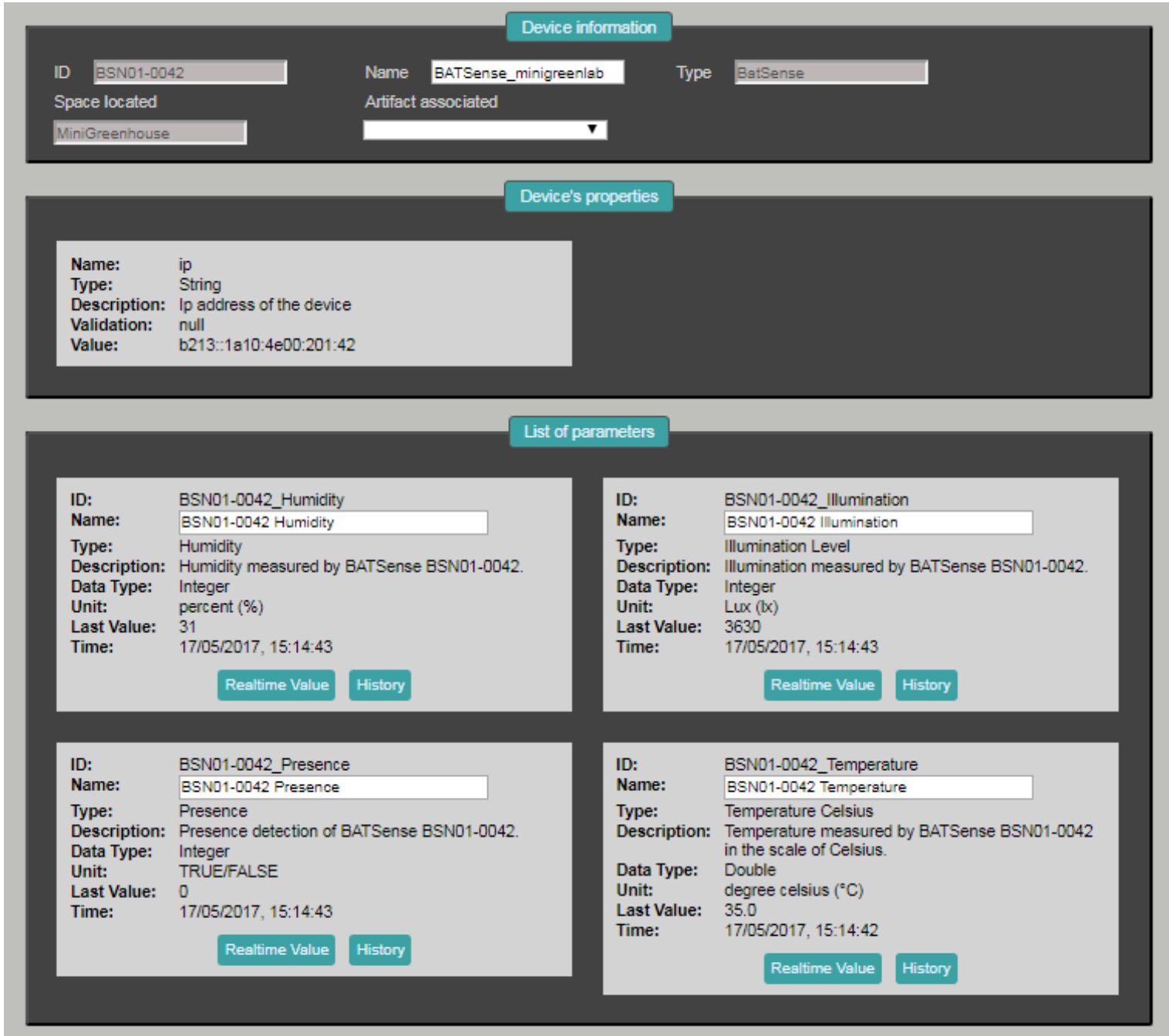

Fig. 6.43 Control panel of device configuration for GreenlabAdmin

\section{Query and control devices through dynamic constructed control panel}

Through the control panel of each device, the user can read the latest status of each belonging parameter of the device and control the device through changing the value of the parameters. Each parameter and its value is shown together with the semantic description including the parameter type, unit, symbol of the unit and the description, which have been provided by the BATOnt. 
The panel is dynamically constructed according to the attributes of the parameter. Different buttons for actions are displayed according to the attributes AccessType, modifiable and DataType of each parameter:

- If the AccessType contains the value "Request", which indicates that the parameter can be queried in real time, then a button "Realtime Value" is displayed.

- If modifiable is true, which indicates that the parameter corresponding to an actuator function of the device, then a button "Set new Value" is displayed.

- A button "History" is displayed for consulting the historical data for those parameters that do not have String as their DataType.

Ask for realtime value of parameters Fig. 6.44 shows the control panel of device configuration for BATSense_minigreenlab for Johnny. 


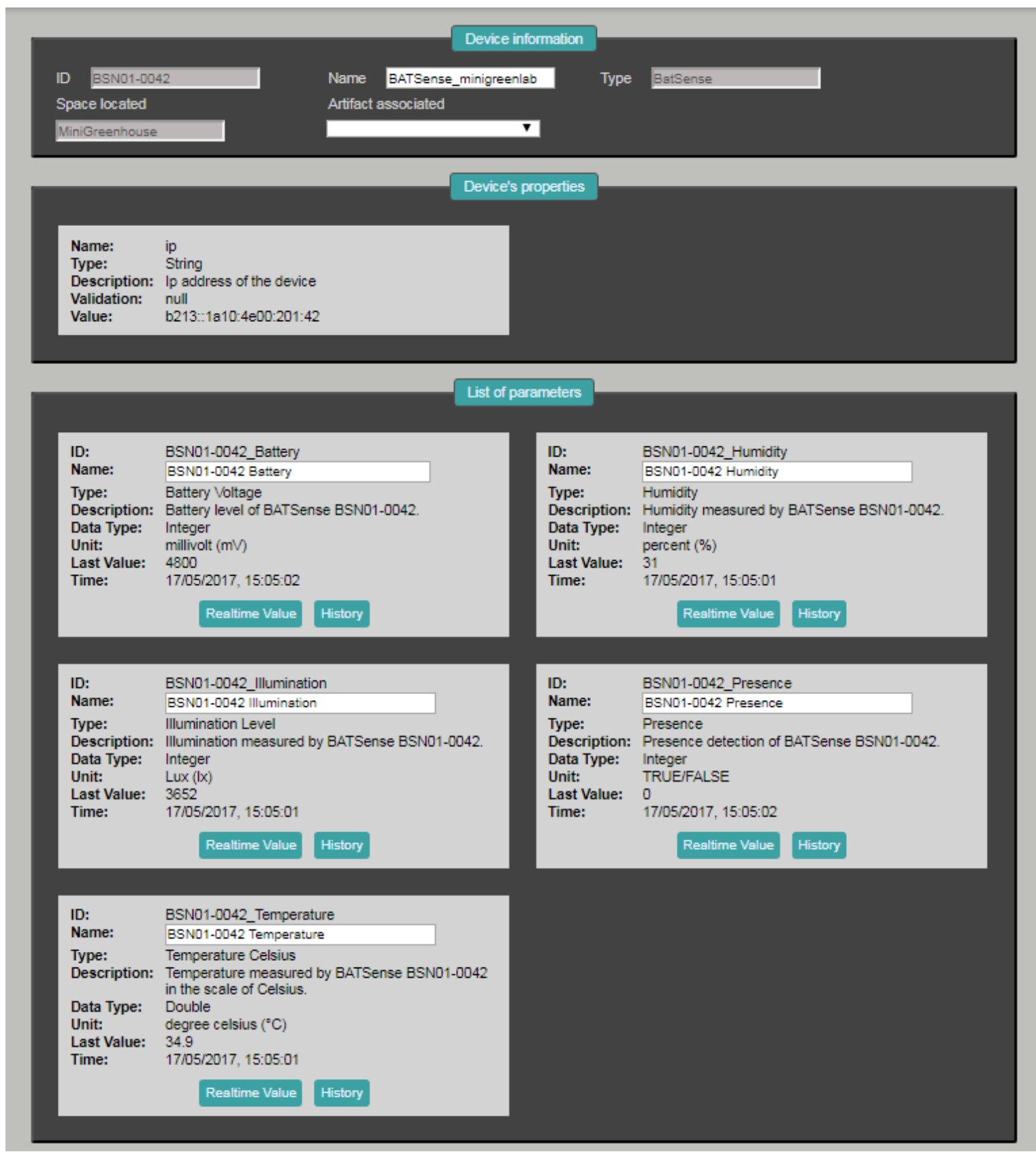

Fig. 6.44 Control panel of device configuration for BATSense

By clicking the button "Realtime Value", a new panel is shown, as illustrated in Fig. 6.45. This panel allows the user to communicate with the device in real time and query the value. 


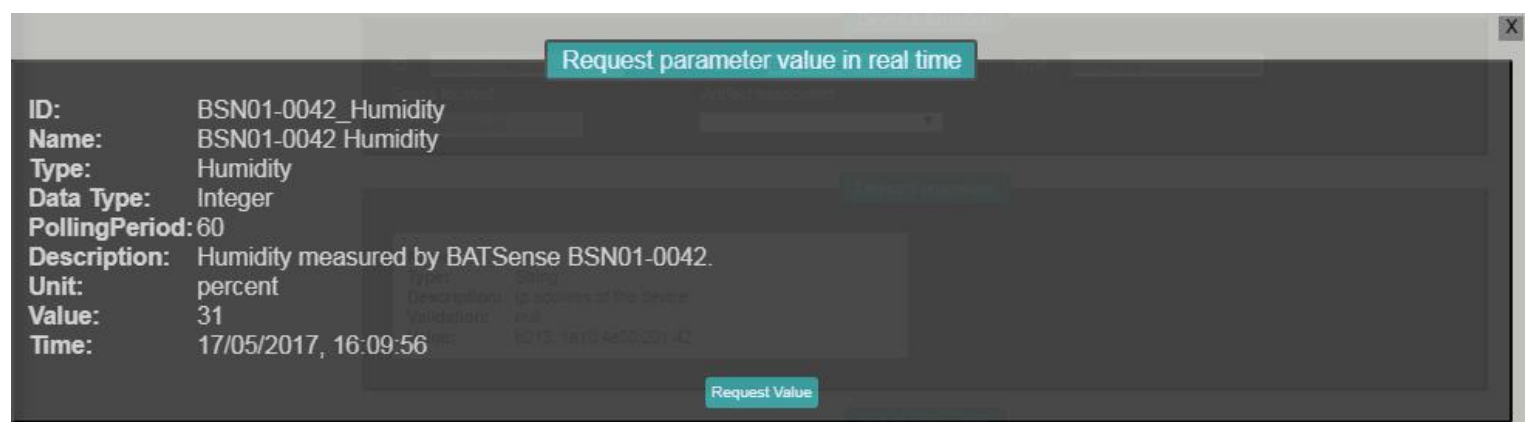

Fig. 6.45 Control panel for requesting parameter value in real time

In the illustrated example, a GET request with path "/parameter/local/realtime/BSN010042_Humidity" is sent to the Central Server when the button "Request Value" is clicked. The Central Server then communicates with the corresponding Border Router and query for the realtime value of this parameter. This value represents the status of the device at the moment, which will not be stored in the Data Warehouse.

Set new value of parameters The user can control the device by changing the value of the authorized parameters. Fig. 6.46 shows the control panel of device configuration for the LightActuator. 


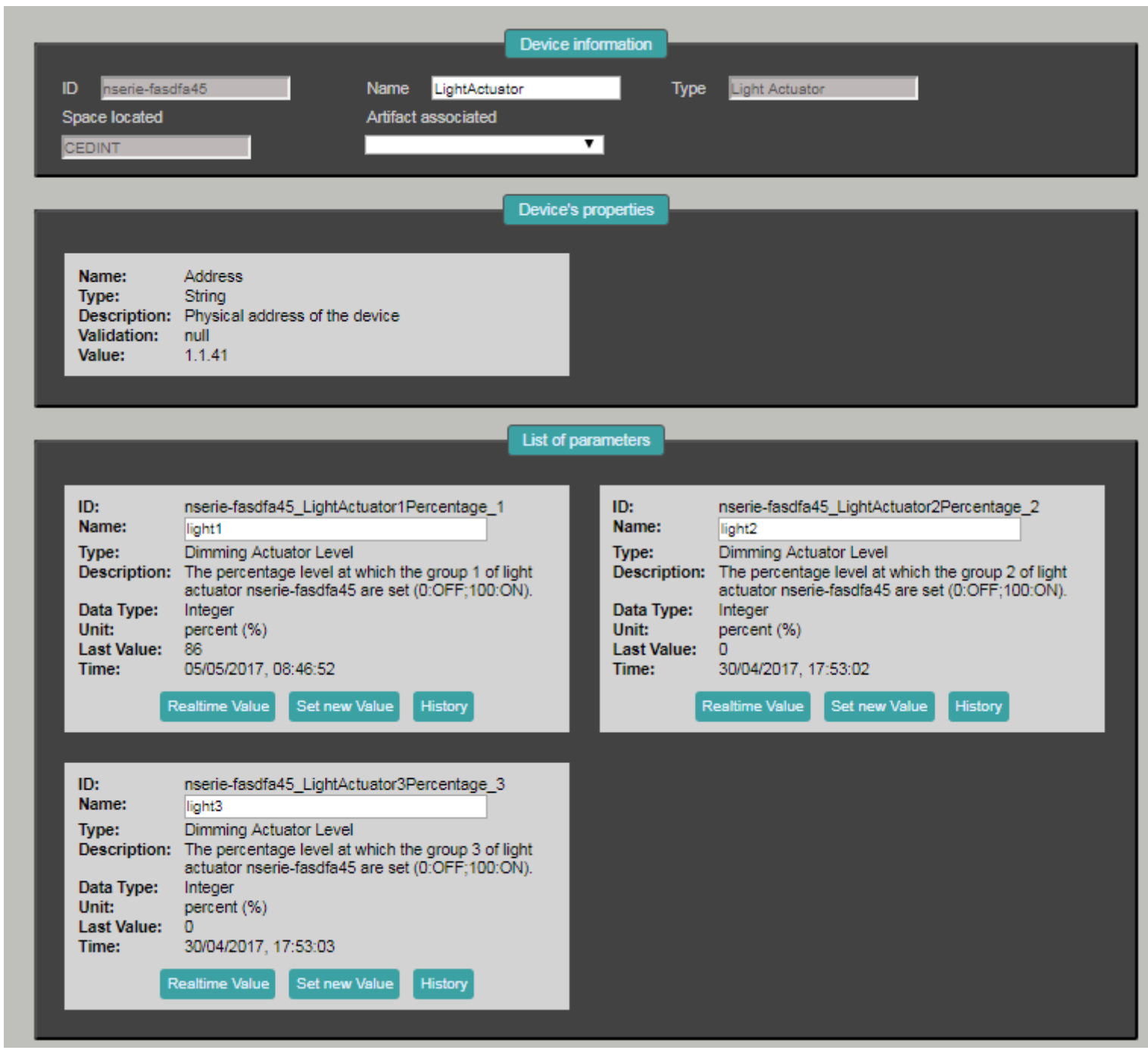

Fig. 6.46 Control panel of device configuration for LightActuator

By clicking the button "Set new Value", a control panel will be displayed, as shown in Fig. 6.47, which is for setting new value of a modifiable parameter. 


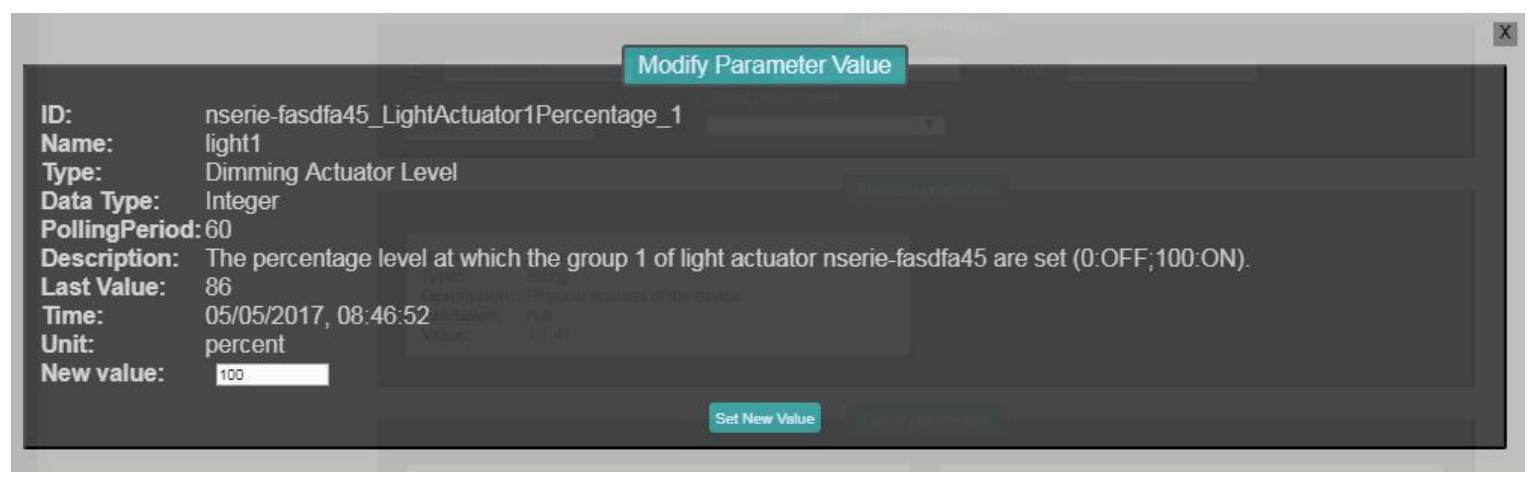

Fig. 6.47 Control panel for modifying parameter value

In the illustrated example, by inputting new value in the text field, the user can change the dimming level of the light1. After the button "Set New Value" is clicked, a PUT request with the path "/parameter/local/userUpdate/nserie-glharg45_BlindActuator1Percentage_1" and JSON representation $\{$ "setValue":" $\{100\} "\}$ as the payload will be sent to the Central Server. If the action succeeds, a message indicating that the "The value has been updated successfully." will show and the field Last Value and Time of this parameter will be updated in the control panel.

Consult the historical data If the Data Type of the parameter is Integer, Double or Boolean, the user can consult its historical data in the form of graph. Fig. 6.48 shows the control panel for historical measurements for BDM01-0041_Dimmer. Since all the value of the measurements are returned as String, the consumer of the Web API should parse the values according to the DataType of the parameter. 


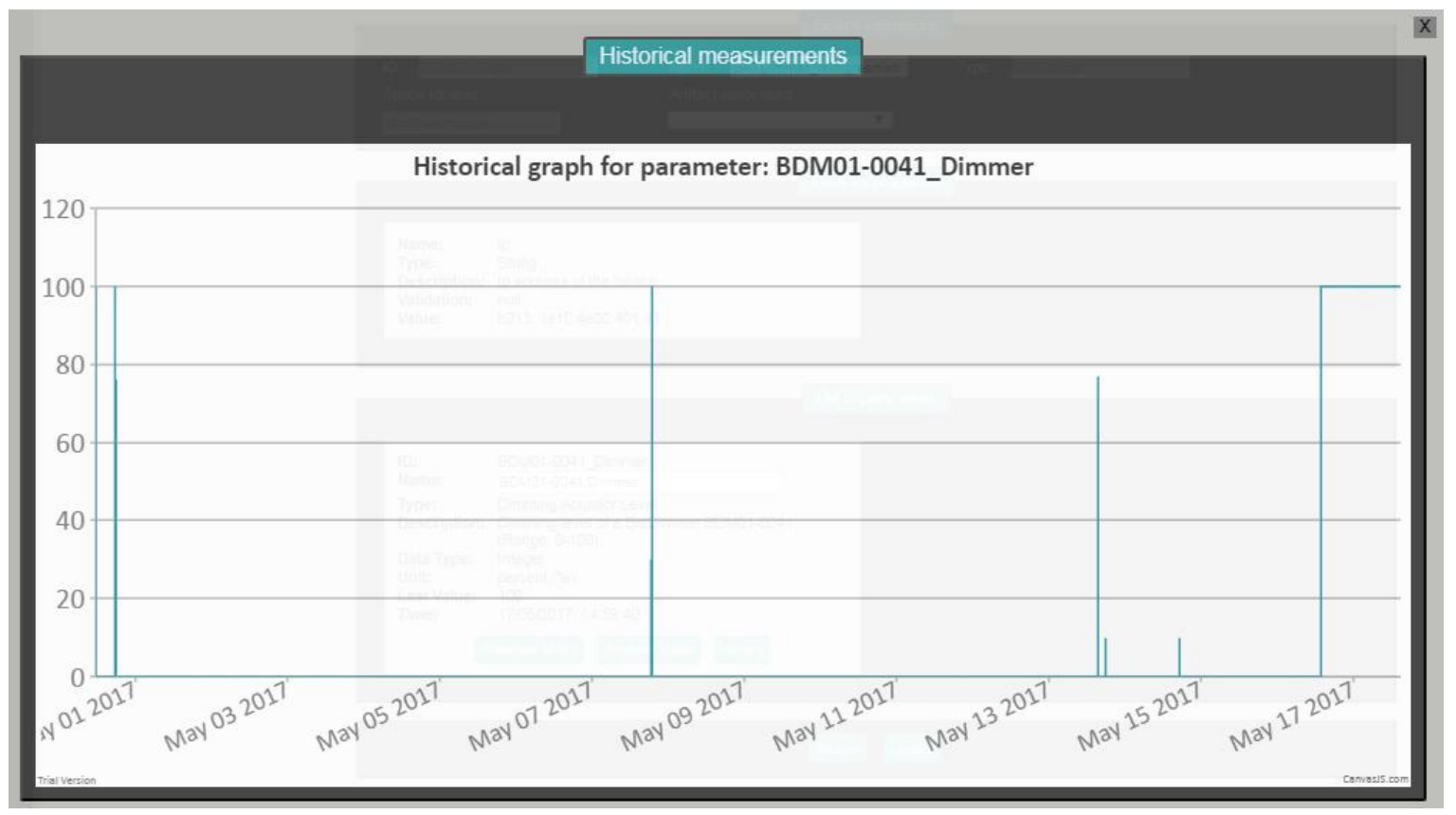

Fig. 6.48 Control panel of historical measurements

\section{Control device through an application}

Instead of changing the status of the devices through the device configuration panel, the user can also control the authorized devices through applications, which have more friendly user interfaces and with more customized information. After choosing the application, the user will be redirected to the corresponding application. Fig. 6.49 shows the homepage of the installed application GreenLab, which is used to monitor and control the mini greenhouse. The interface has been designed by the Energy Efficiency group of CeDInt. 


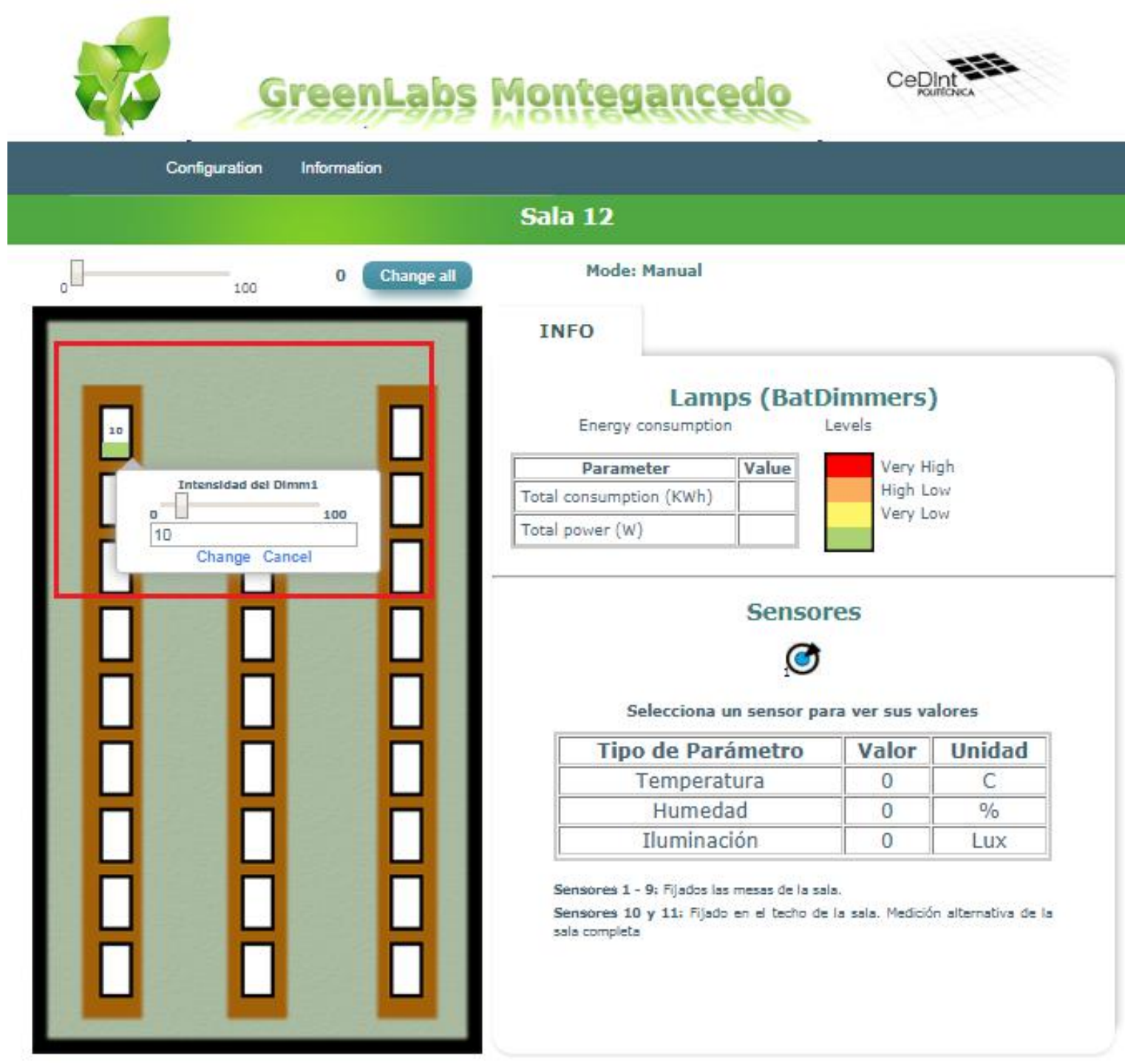

Fig. 6.49 User interface of application Greenlab for Mini Greenhouse

By selecting the corresponding dimmer, the user can change the dimming level of the BATDimmer_minigreenlab. As shown above, the web-based interface provides users a visualized and convenient way to interact with the platform.

\subsubsection{External application based user interface}

Apart from the web-based user interface, the implemented Web API also allows the integration with third-party applications. By doing this, the user can realize the interaction with the platform through more familiar and convenient interfaces. 


\section{D-model based user interface}

By using the Web API that has been developed, a 3D-model based user interface can communicate with the IoT platform to carry out the control of devices. Fig. 6.50 shows an 3D-model for the control of showroom, which has been developed by the Virtual Reality Research Group of CeDInt. The interface was developed in Unity, which is a cross-platform game development platform which is used for buding high/quality 3D and 2D games. Through this interface, the user is able to first see the simulation result of the operations and then issue a request for changing the status of a device.

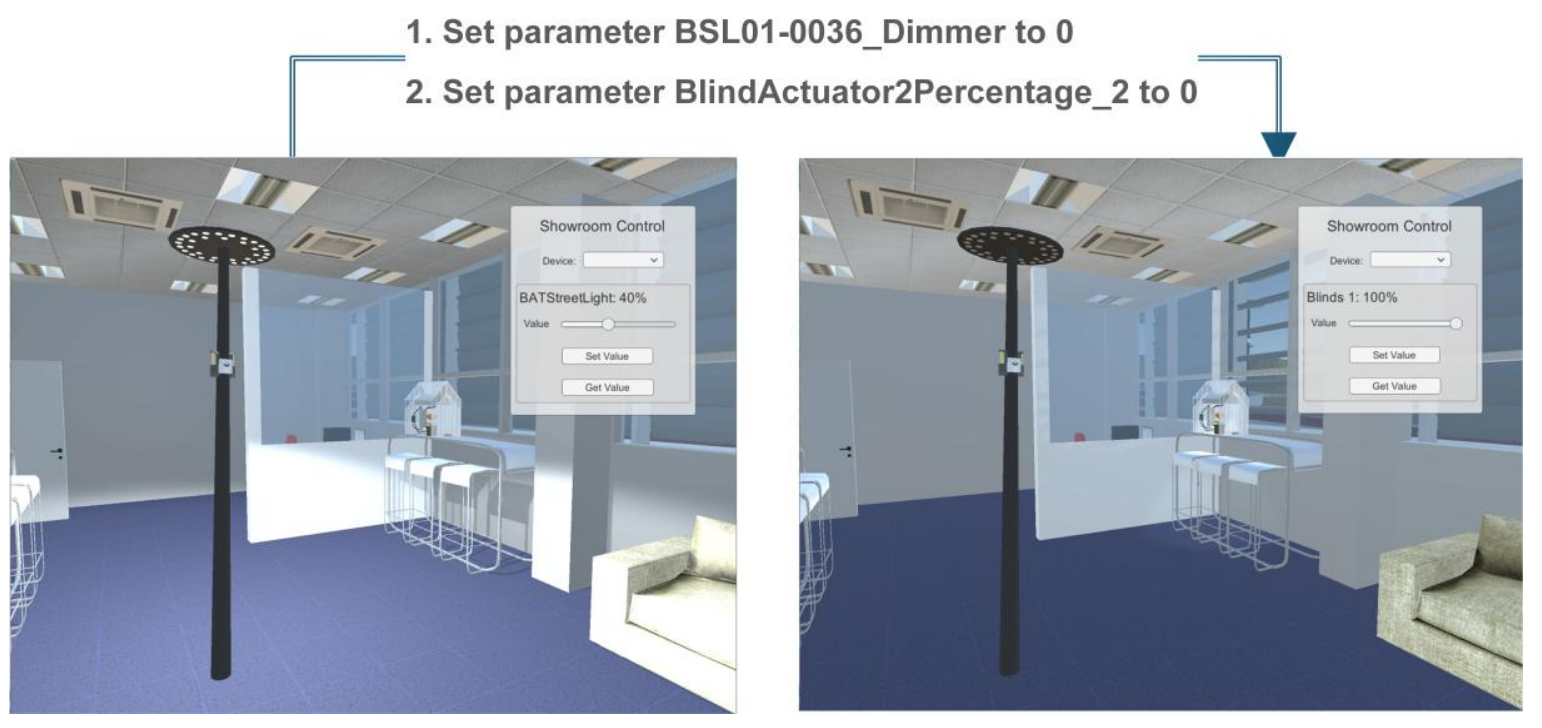

Fig. 6.50 3D-model based user interface for showroom control implemented in Unity

Fig. 6.51 shows the workflow of the integration of the 3D-model based user interface with the developed IoT platform. 


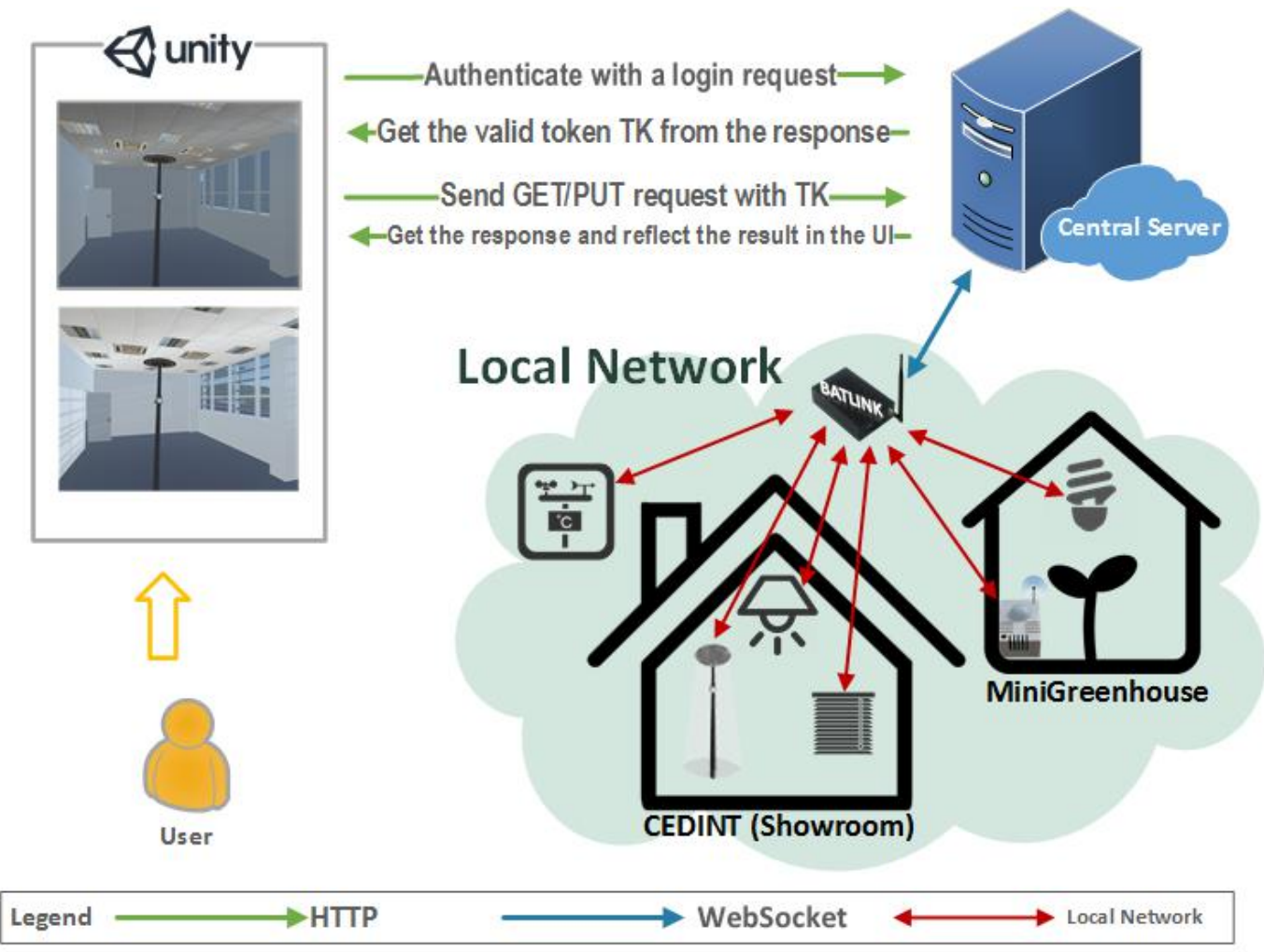

Fig. 6.51 Workflow of the integration with 3D-model based user interface

When the program of the user interface is executed, a login request is sent first to the Central Server for getting a valid token TK and store it locally. The stored token TK should be included in the Authentication header in all the following request. If the request is rejected, a login request should be sent again for getting a new TK. The program currently works with the account of Johnny as its default account.

Set Value A user can select a device and change its value. The program will first show a simulation of the result for setting the new value. When the user is satisfied with the result of the simulation, he can send a request to control the device by pressing the button "Set Value", the program will then send the request to the Central Server through the provided Web API. A PUT request will be sent with the path "/parameter/local/userUpdate/ $\left\{\mathrm{ID}_{\text {parameter }}\right\}$ ", where $\mathrm{ID}_{\text {parameter }}$ indicates the ID of the target parameter. The value to set should be included in the payload in the form of JSON object $\{$ "setValue":" $\{$ newValue $\}$ " $\}$. The Central Server will communicate with the corresponding Border Router to execute the command in the local network and then return the result to the Unity program. The program will reflect the result in the 3D-Model. 
Get Value If the button "Get Value" is pressed, a GET request will be sent to the Central Server with path "/parameter/ $\left\{\mathrm{ID}_{\text {parameter }}\right\}$ ", where $\mathrm{ID}_{\text {parameter }}$ indicates the ID of the target parameter. By receiving the JSON representation of the target parameter from the Central Server, the program can retrieve the last value of the parameter and reflect it in the 3D-Model.

In this demonstration, the 3D-Model communicates with the developed IoT platform to control the devices that use different communication protocols, including:

- BATStreetLighting and BATDimmer, which communicate through the CoAP/6LoWPAN protocols

- BlindActuator and LightActuator, which communicate through the KNX protocol

\section{WeChat-based user interface}

WeChat is a Chinese social media application which provides services of instant messaging. WeChat was first released in 2011 and by 2016 it was one of the largest standalone messaging apps by monthly active users with over 889 million active users spending 66 minutes a day on the app on average. Apart from the normal instance messaging service, WeChat also provides an Admin Platform which can be registered by cooperation or individuals to promote their brand or service to billions of WeChat users. To provide service through the WeChat Admin Platform, a cooperation or an individual needs to first register an official account and complete the configuration through the control panel. In the demonstration, an official account has been registered with name CoolHouse and a CoolHouse Server has been implemented. The CoolHouse Server interprets the the messages received from the WeChat Server and communicates with the Central Server through the Web API for controlling the devices in the test bed. The workflow and the message exchanged is illustrated in Fig. 6.52. 


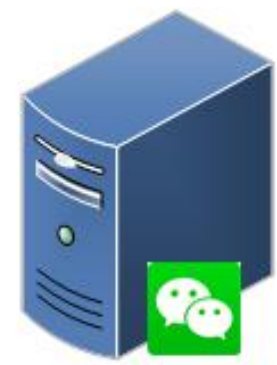

WeChat Server
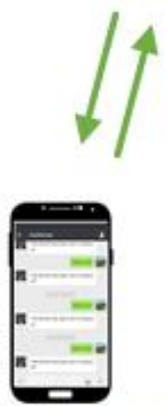

We Chat APP

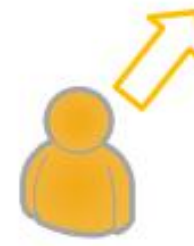

User

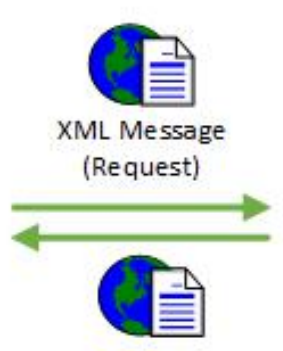

XML Message

(Response)

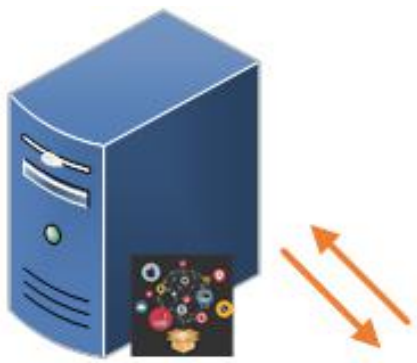

CoolHouse Server

\section{Central Server}

Legend

HTTP Request

HTTP Request

(Web API)

Local network

Fig. 6.52 Workflow of integration of WeChat Admin Platform with the platform

The integration of WeChat Admin Platform with the platform involves five steps:

[1] As long as a user send a sentence to the account CoolHouse from the WeChat APP, the message will be sent to the WeChat Server through the WeChat API.

[2] The WeChat Server wraps the sentence in an XML message along with the toUserName, FromUserName, createTime and forward it to the CoolHouse Server. Fig. 6.53 shows an example of the XML message [128]

[3] After receiving the message, the CoolHouse Server retrieves the information and issues a corresponding request to the platform through the developed Web API and wait for the response. 
[4] The platform handles the request internally and return the result of the action to the CoolHouse Server.

[5] The CoolHouse Server interprets the result and generates an response XML Message to the WeChat Server. The XML Message contains the reply sentence which will be shown to the user in the conversation.

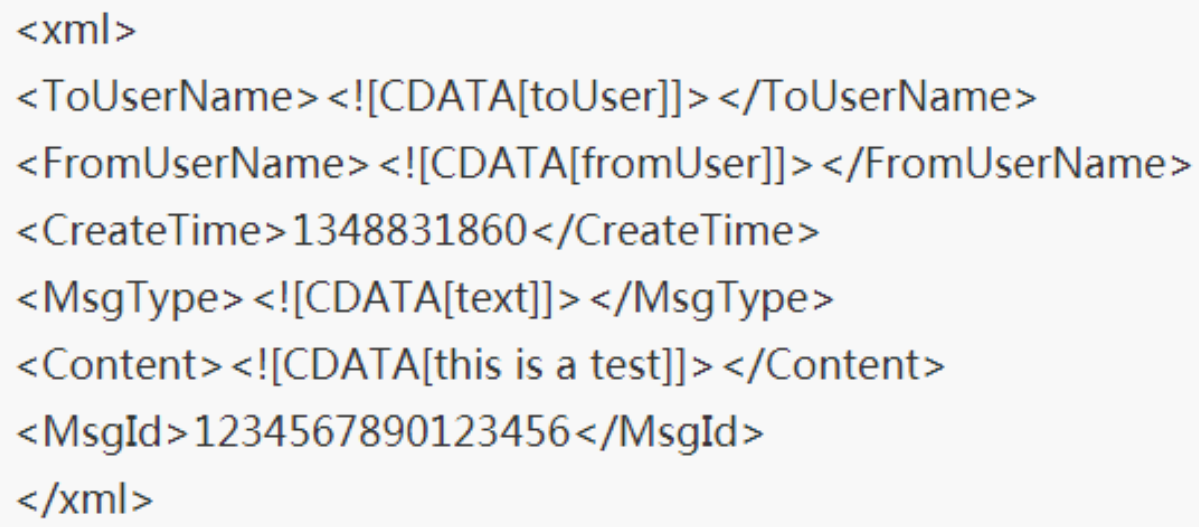

Fig. 6.53 Example WeChat message and format

Where each attribute refers to:

- ToUserName, Developer's WeChat ID

- FromUserName, Unique sender ID (an OpenID)

- CreateTime, Message creation time (Integer)

- MsgType, Text

- Content, Message content

- MsgId,A unique message ID (64 bit integer)

Fig. 6.54 shows the control panel for official account settings for CoolHouse. Apart from the general information such as profile photo, region, a QR code is provided which can be scanned by the users for subscribing to the account. 


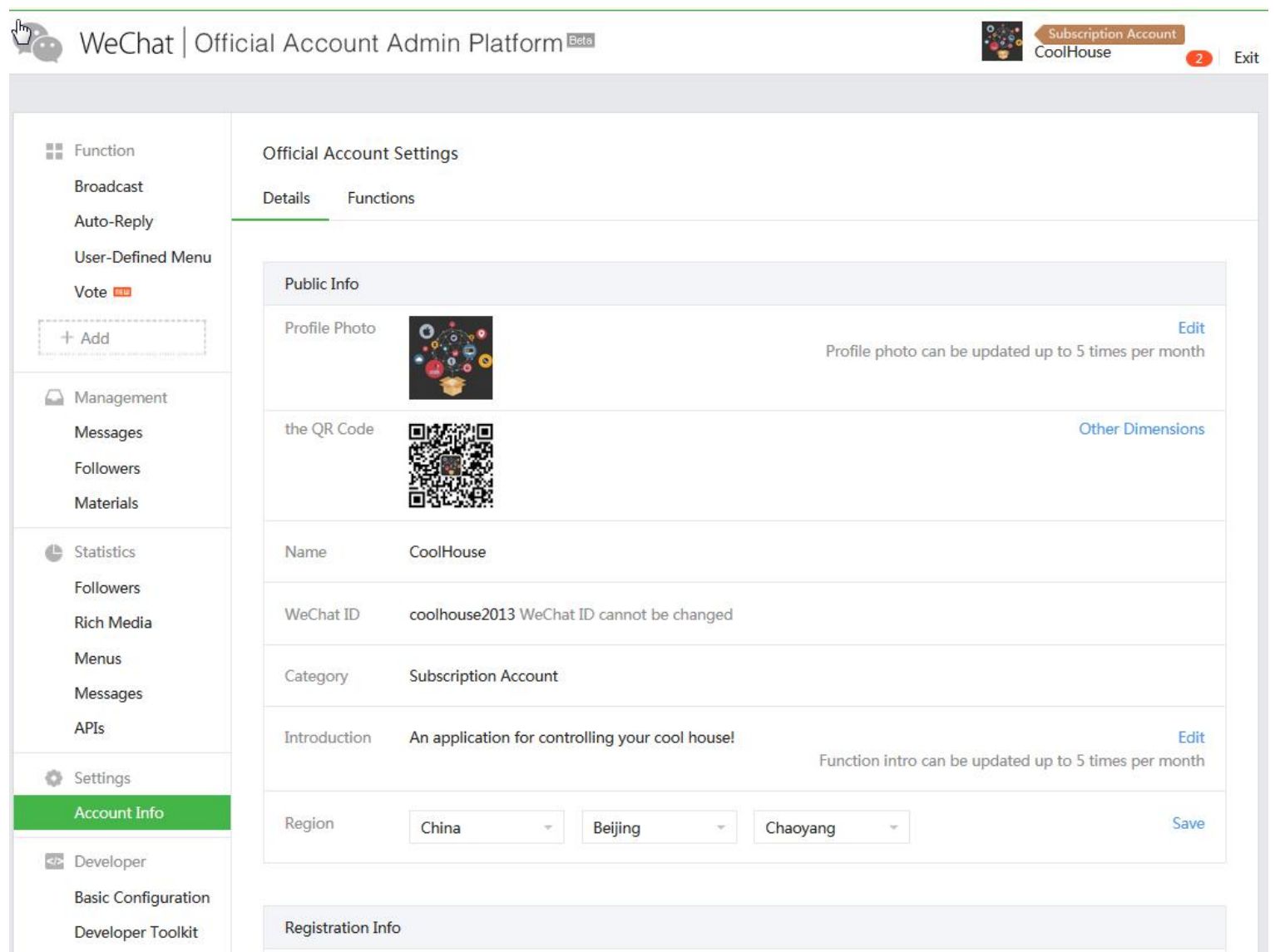

Fig. 6.54 Control panel for official account setting in WeChat Admin Platform

Fig. 6.55 shows the control panel for server configuration for CoolHouse, where the address of the CoolHouse Server should be indicated. 


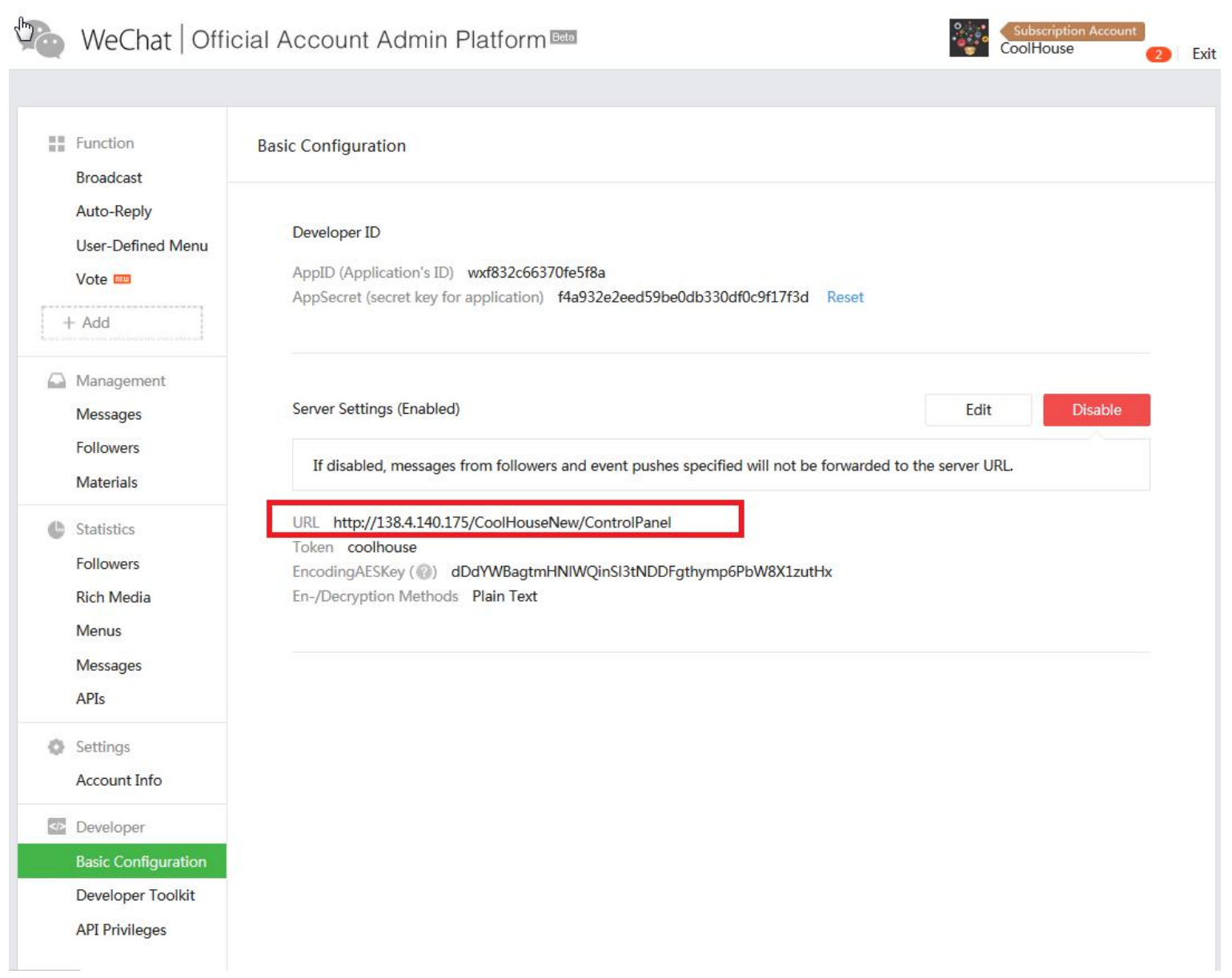

Fig. 6.55 Control panel for server configuration in WeChat Admin Platform

From the perspective of users, to use the service provided by the official account, they need to first subscribe to the account CoolHouse. Fig. 6.56 shows the procedures of subscription. The user can subscribe to the account either by searching in the public list of official accounts or scanning the corresponding QR code. 


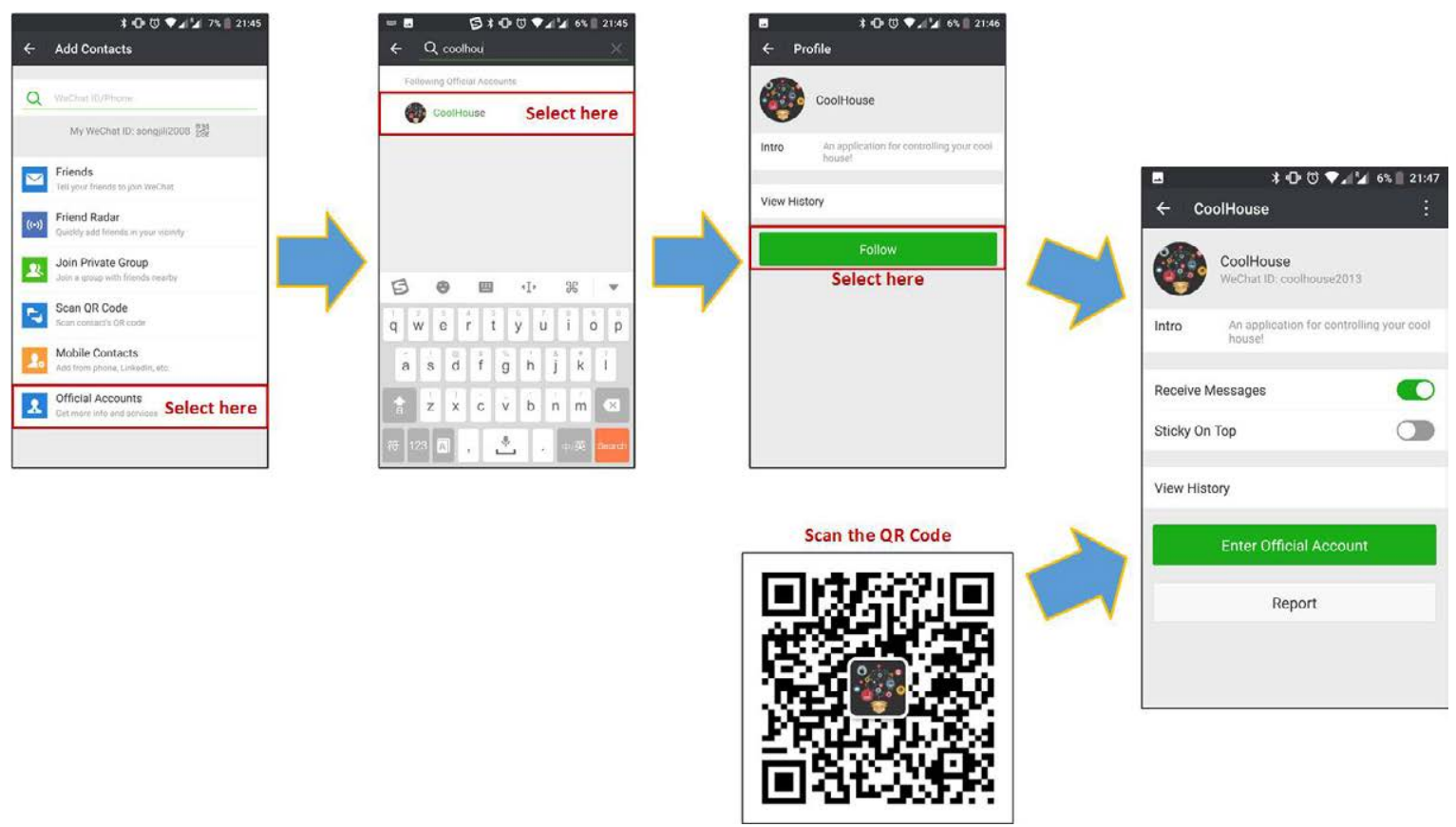

Fig. 6.56 Subscribe to the official account CoolHouse

As long as the user subscribes to the account successfully, the user can start to control the devices in the test bed through the conversation with the account. As shown in Fig. 6.57, when the user says "blind 4 on" to the account CoolHouse, the sentence will be wrapped in an XML message and sent to the CoolHouse Server. After receiving the message, the CoolHouse Server analyses the message and send a request accordingly to Central Server. The Central Server makes corresponding actions and returns the result to the CoolHouse Server. 


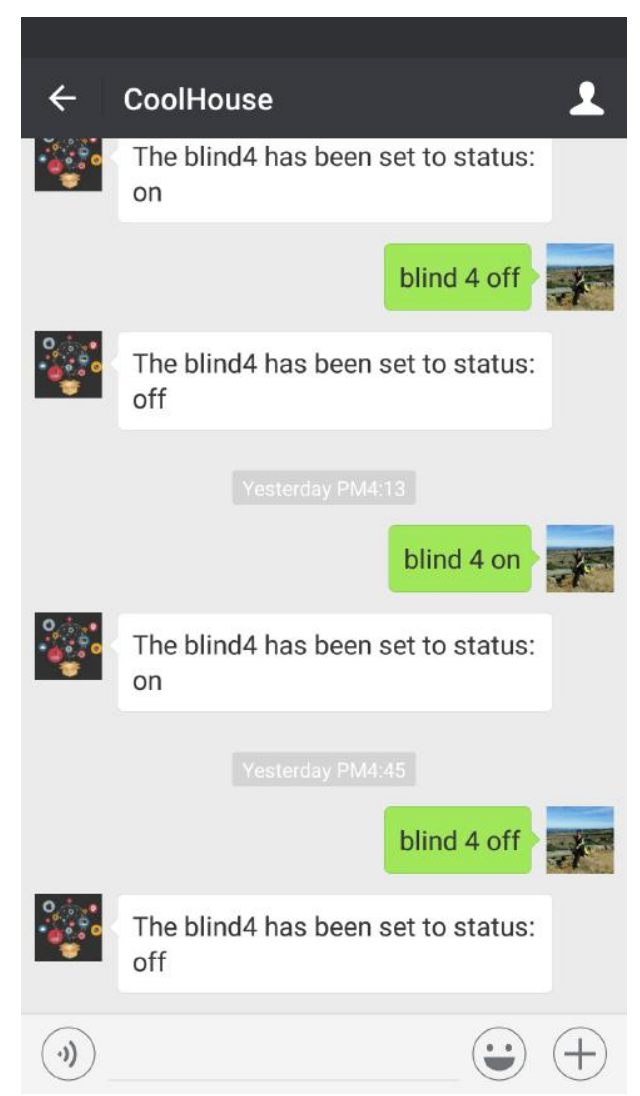

Fig. 6.57 Talk with the official account CoolHouse for controlling devices in test bed

In the illustrated case, after receiving the command "blind 4 on" in the chat conversation:

[1] The WeChat Server wraps the command in an XML message and send it to the CoolHouse Server.

[2] The CoolHouse Server then sends a PUT request to the Central Server to fully open the No.4 blind, which includes:

- Path: "/parameter/local/userUpdate/nserie-glharg45_BlindActuator4Percentage_4"

- Payload: $\{$ "setValue":"0"\}

[3] If the action is performed successfully in the developed IoT platform, a success message will be returned to the CoolHouse Sever. Otherwise, an error message will be returned.

[4] The CoolHouse Sever converts the message into a conversation sentence "The blind4 has been set to status: on".

[5] The CoolHouse Server wraps the sentence in an XML message, as illustrated in Fig. 6.58, and sends it to the WeChat Server for returning it to the final user. 


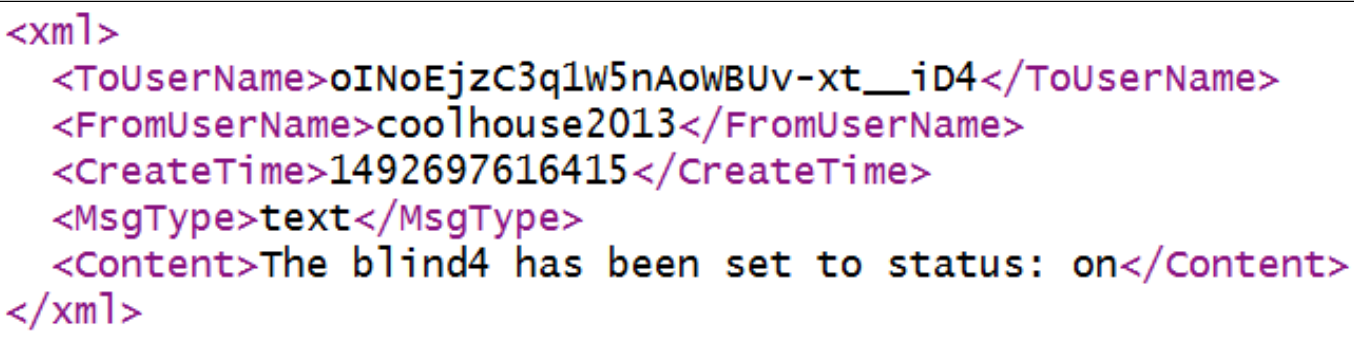

Fig. 6.58 WeChat message sent from CoolHouse Server to WeChat Server

By using WeChat as the interface, the users can interact with Things through a familiar and comfortable conversation, which improves the user experience. 



\section{Chapter 7}

\section{Conclusion and future work}

\subsection{Conclusion}

This thesis has addressed a research about the data management in a open IoT platform combining the Semantic Web technologies and standard Web protocols, obtaining a semantic interoperable, flexible and scalable IoT solution. In summary, the following contributions have been accomplished.

- A mechanism has been designed which uses a concept parameter to interpret the functional behaviour of the Things. The parameters have been used to exchange and synchronize information among users, devices and applications. This mechanism facilitates the integration of new devices into the system and allows the comparison of the same functionalities among different devices.

- An annotation-based mechanism has been designed to carry out the interaction with the platform knowledge base. This work helps to store the data simultaneously in both of a relational database and a semantic triple store according to their properties, ensuring the data consistency between the two different data storage.

- An ontology BATOnt has been created, which provides a common vocabulary for the domain of IoT. The vocabulary is for describing not only the Things, but also the related concepts that could be involved in an IoT project implementation, which are about people, spaces, control and authorization. This improves the semantic interoperability of the IoT by providing a schema for the semantic description of functionality of Things and the data they generated, which helps to share a consensus among the device manufactures, application developers, data scientists and end users. 
- A Web API has been developed for carrying out the communication between agents, either users or border routers, with the IoT platform. A guidance has been provided for the construction of the Web API. By using the designed API, users can realize the interaction with Things through a standard Web-based interface regardless their own communication protocols.

- A fine-grained access control mechanism has been designed to ensure the security and privacy, which can dynamically decide the authorities of users to parameters, devices and spaces according to the configuration of roles and permissions. The mechanism works on basis of a new proposed concept "Private Knowledge Graphs"(PKG). PKG is a subset of the System KG, which is generated by performing inference with a set of rules for fact and trust policies and the relationships of user-role, role-parameters that are already stated in the system knowledge base. It contains exclusively the information that an agent is authorized to access. The designed mechanism helps to deal with the situation of a "multi-client, multi-user" system and restricts the users to only access the authorized resources on the basis of Web API and PKGs.

- A case study has been given to illustrate the working mechanism of the designed access control system. Moreover, a demonstration has been implemented to show the use of the designed system in practical cases. The demonstration has tested the different manners to interact with the developed IoT platform through the designed Web API, including the Postman REST Client, the implementation of a Web-based user interface. In addition, the demonstration includes the integration with two thirdparty applications, a 3D-Model based user interface and a chat application to provide user more familiar and convenient interfaces, which have validated the possibility of creating mashup applications on the basis of the developed IoT platform.

\subsection{Discussion and future work}

In this thesis, an ontology BATOnt has been created for providing a common vocabulary in the domain of IoT. As shown in the LinkedData[38], more and more ontologies have been created and connected into the LinkedData set. In the practical cases, it is not easy to create an ontology and convince others to use it in their existing system. The users and developers need to consider about an amount of problems, such as the cost of the immigration, the demand of their organization. As a result, to promote an semantic solution for data management, apart from the creation of an ontology, a corresponding framework is also in demand for the interaction with the knowledge base. By providing a flexible solution with easy-to-use Web 
interfaces and good examples, the easy integration with other semantic vocabularies and IoT platforms is allowed.

\subsubsection{Integration with other semantic vocabularies}

Integrating with the widely used semantic vocabularies from both research organizations and commercials can help BATOnt to be used by more users in the practical cases. Schema.org[114] is a shared vocabulary developed and used widely by Google, Microsoft, Yahoo and Yandex and can be easily extended through a well-documented extension model. With the help of rich snippets from Google, the websites can share and publish their data in the google search result by adding structured data mark up in their web content, using the vocabulary provided by Schema.org. As a result, creating an extension of Schema.org on the basis of BATOnt which contains the IoT data such as devices, communication protocols, building infrastructures would provide a practical way to publish structured IoT data in the World Wide Web. By making use of the created extension and the APIs provided by the Google rich snippets and BATMP, a search engine for IoT Linked Data can be implemented.

\subsubsection{Integration with other IoT platform}

With more and more Things connected into the developed IoT platform, a big amount of data is generated. By sharing the data with other IoT platform, the data can be unified with the data collected from other data sources for the further data mining. So far, some tests have been conducted between the developed platform and other IoT platforms such as Carriots[129], SOFIA2[130], FIWARE[131] and IBM Watson[132]. In normal cases, the developed IoT platform can submit data to other IoT platform through the developed Web API. However, in some cases, if the platform makes use of specific protocols, an adapter should be implemented for the bridging between the different protocols. For instance, IBM Watson uses MQTT as the communication protocol, as a result, an adapter needs implemented, which inquires data from the developed IoT platform and sends it to the IBM Watson through MQTT.

\subsection{Publications}

\section{Journal}

[1] Jaime Caffarel, Song Jie, Jorge Olloqui, Rocío Martínez, Asunción Santamaría, "Implementation of a Building Automation System based on Semantic Modeling", Journal 
of Universal Computer Science. Special issue Towards Sustainable Computing through Ambient Intelligence. 2013

\section{Conference}

[1] Jie Song, Silvia Calatrava Sierra, Jaime Caffarel Rodríguez, Jorge Martín Perandones, Guillermo del Campo Jiménez, Jorge Olloqui Buján, Rocío Martínez García and Asunción Santamaría Galdón. "Data Consistency Management in an Open Smart Home Management Platform", EMS2014, European Modelling Symposium 2014, 21-23 October, 2014, Pisa, Italy

[2] Jorge M. Perandones, Guillermo del Campo Jiménez, Jaime Caffarel Rodríguez, Song Jie, Silvia Calatrava Sierra, Rocío Martínez García, "Energy-saving Smart Street Lighting System based on 6LoWPAN". The first international conference on IoT in Urban Space, 27-28 October, 2014, Rome, Italy.

[3] Jie Song, Silvia Calatrava Sierra, Jaime Caffarel Rodríguez, Jorge Martín Perandones, Guillermo del Campo Jiménez, Jorge Olloqui Buján, Rocío Martínez García and Asunción Santamaría Galdón. "Parameter-based Mechanism for Unifying User Interaction, Applications and Communication Protocols", Second international conference on Artificial Intelligence, Modelling and Simulation, 18-20 November 2014, Madrid, Spain

[4] Jorge Marín, Igor Gómez, Eduardo Montoya, Jie Song, Jorge Olloqui, Rocío Martínez, "BatNet: an implementation of a 6LoWPAN sensor and actuator network", e-Energy'13, Berkeley, CA, USA, 21 May. 2013

[5] Jaime Caffarel, Song Jie, Jorge Olloqui, Rocío Martínez "Bat-MP: An ontologybased energy management platform", UCAmI 2012: VI International conference on Ubiquitous Computing\&Ambient Intelligence., Vitoria, España, 2012

\subsection{Projects}

- Plataforma de Decisión para Gestión de Edificios Inteligentes mediante Control por Geoposicionamiento (EGEO) [IPT-2012-0552-380000]. MINECO. 2012-2015 . (Building Automation)

- I2TECH CEI Montegancedo: Mejora del entorno del campus. [CEI10/00041]. MECD/ MINECO. 2013-2016. (Smart Lighting, Greenhouses, Building Automation) 
- All IP Open Solution for IoT on 4G/5G Mobile Networks (ALLIPOP) [TSI-1001022015-9] MINETUR. 2015-2018. (Smart Lighting) 



\section{References}

[1] How X10 Works. http://www.smarthomeusa.com/how-x10-works/.

[2] Hermann Merz, Thomas Hansemann, and Christof Hübner. Building Automation: Communication Systems with EIB/KNX, LON and BACnet. Springer Science \& Business Media, 2009.

[3] Dietmar Loy, Dietmar Dietrich, and Hans-Joerg Schweinzer. Open control networks: LonWorks/EIA 709 technology. Springer Science \& Business Media, 2012.

[4] Geoff Mulligan. The 6LoWPAN architecture. In Proceedings of the 4th workshop on Embedded networked sensors, pages 78-82. ACM, 2007.

[5] Carles Gomez, Joaquim Oller, and Josep Paradells. Overview and evaluation of bluetooth low energy: An emerging low-power wireless technology. Sensors, 12(9):11734$11753,2012$.

[6] Khusvinder Gill, Shuang-Hua Yang, Fang Yao, and Xin Lu. A zigbee-based home automation system. IEEE Transactions on consumer Electronics, 55(2), 2009.

[7] Z-Wave the Smartest Choice for your Smart Home. http://www.z-wave.com/.

[8] Somayya Madakam, R Ramaswamy, and Siddharth Tripathi. Internet of things (iot): A literature review. Journal of Computer and Communications, 3(05):164, 2015.

[9] L Ericsson. More than 50 billion connected devices. White Paper, 2011.

[10] The Web of Things with Mozilla Open Badges. https://www.slideshare.net/ PatrickJohnMcGee/the-web-of-things-with-mozilla-open-badges- 40521825.

[11] Web of Things-Architecting the Web of Things, for techies and thinkers! https: //webofthings.org/.

[12] Dominique Guinard, Vlad Trifa, Thomas Pham, and Olivier Liechti. Towards physical mashups in the web of things. In Networked Sensing Systems (INSS), 2009 Sixth International Conference on, pages 1-4. IEEE, 2009.

[13] Dominique Guinard, Vlad Trifa, and Erik Wilde. A resource oriented architecture for the web of things. In Internet of Things (IOT), 2010, pages 1-8. IEEE, 2010.

[14] Vlad Mihai Trifa. Building blocks for a participatory web of things: devices, infrastructures, and programming frameworks. PhD thesis, ETH Zurich, 2011. 
[15] Dominique Guinard. A Web of Things Application Architecture - Integrating the Real-World into the Web. PhD thesis, ETH Zurich, Zurich, Switzerland, August 2011.

[16] W3C Web of Things at W3C. https://www.w3.org/WoT/.

[17] WoT Current Practices. https://w3c.github.io/wot/current-practices/wot-practices. html.

[18] Extensible Markup Language (XML). https://www.w3.org/XML/.

[19] JSON. http://www.json.org/.

[20] Web Services Glossary. https://www.w3.org/TR/2004/NOTE-ws-gloss-20040211/ \#webservice.

[21] Don Box, David Ehnebuske, Gopal Kakivaya, Andrew Layman, Noah Mendelsohn, Henrik Frystyk Nielsen, Satish Thatte, and Dave Winer. Simple object access protocol (SOAP) 1.1, 2000.

[22] SOAP Specifications. https://www.w3.org/TR/soap/.

[23] Dominique Guinard, Iulia Ion, and Simon Mayer. In search of an internet of things service architecture: REST or WS-*? A developers' perspective. In International Conference on Mobile and Ubiquitous Systems: Computing, Networking, and Services, pages 326-337. Springer, 2011.

[24] Roy Thomas Fielding. Architectural styles and the design of network-based software architectures. PhD thesis, University of California, Irvine, 2000.

[25] Roy T Fielding. REST APIs must be hypertext-driven. Untangled musings of Roy T. Fielding, 2008.

[26] Jersey. https://jersey.github.io/.

[27] Unirest - Simplified, lightweight HTTP request client libraries in multiple languages. http://unirest.io/.

[28] Google Code Archive - Long-term storage for Google Code Project Hosting. https: //code.google.com/archive/p/chrome-rest-client/.

[29] Postman/Supercharge your API workflow. https://www.getpostman.com/.

[30] RESTClient, a debugger for RESTful web services. https://addons.mozilla.org/es/ firefox/addon/restclient/.

[31] WebSockets-Glossary I MDN. https://developer.mozilla.org/en-US/docs/Glossary/ WebSockets.

[32] RFC 6455-The WebSocket Protocol. https://tools.ietf.org/html/rfc6455.

[33] Thomas R Gruber. Toward principles for the design of ontologies used for knowledge sharing? International journal of human-computer studies, 43(5-6):907-928, 1995. 
[34] N BorstW. Construction of engineering ontologies. PhD thesis, University of Twenty, Enschede, Center for Telematica and Information Technology, 1997.

[35] Rudi Studer, V Richard Benjamins, and Dieter Fensel. Knowledge engineering: principles and methods. Data \& knowledge engineering, 25(1-2):161-197, 1998.

[36] Diana Man. ONTOLOGIES IN COMPUTER SCIENCE. DIDACTICA MATHEMATICA, , 1(1):43-46, 2013.

[37] Christian Bizer, Tom Heath, and Tim Berners-Lee. Linked data-the story so far. Semantic services, interoperability and web applications: emerging concepts, pages 205-227, 2009.

[38] Linked Data I Linked Data - Connect Distributed Data across the Web. http://linkeddata. org/.

[39] Frederick Hayes-Roth, Donald Waterman, and Douglas Lenat. Building expert systems. 1984.

[40] Ganggao Zhu and Carlos A Iglesias. Computing Semantic Similarity of Concepts in Knowledge Graphs. IEEE Transactions on Knowledge and Data Engineering, 29(1):72-85, 2017.

[41] Ian Horrocks. Ontologies and the semantic web. Communications of the ACM, 51(12):58-67, 2008.

[42] Semantic Web - XML2000 - slide "Architecture". https://www.w3.org/2000/Talks/ 1206-xml2k-tbl/slide10-0.html.

[43] Semantic Web, and Other Technologies to Watch: January 2007 (24). https://www.w3. org/2007/Talks/0130-sb-W3CTechSemWeb/\#(24).

[44] RFC 2396-Uniform Resource Identifiers (URI): Generic Syntax. https://tools.ietf.org/ $\mathrm{html} / \mathrm{rfc} 2396$.

[45] RFC 3986-Uniform Resource Identifier (URI): Generic Syntax. https://tools.ietf.org/ $\mathrm{html} / \mathrm{rfc} 3986$.

[46] Technical Introduction. http://www.unicode.org/standard/principles.html.

[47] RFC3987-Internationalized Resource Identifiers (IRIs). https://www.ietf.org/rfc/ rfc3987.txt.

[48] RFC 1738-Uniform Resource Locators (URL). https://tools.ietf.org/html/rfc1738.

[49] Namespaces in XML 1.0 (Third Edition). https://www.w3.org/TR/REC-xml-names/.

[50] RDF-Semantic Web Standards. https://www.w3.org/RDF/.

[51] RDF Schema 1.1. https://www.w3.org/TR/rdf-schema/.

[52] OWL Web Ontology Language Overview. https://www.w3.org/TR/2003/ PR-owl-features-20031215/. 
[53] OWL Web Ontology Language Reference. https://www.w3.org/TR/owl-ref/.

[54] Jeremy J Carroll, Christian Bizer, Pat Hayes, and Patrick Stickler. Named graphs, provenance and trust. In Proceedings of the 14th international conference on World Wide Web, pages 613-622. ACM, 2005.

[55] RDFa 1.1 Primer-Third Edition. https://www.w3.org/TR/rdfa-primer/.

[56] Apache Jena-Home. https://jena.apache.org/.

[57] Apache Jena-TDB. https://jena.apache.org/documentation/tdb/.

[58] OpenLink Software Home Page. https://www.openlinksw.com/.

[59] Amit Singhal. Introducing the knowledge graph: things, not strings. Official google blog, 2012.

[60] Nathan Bronson, Zach Amsden, George Cabrera, Prasad Chakka, Peter Dimov, Hui Ding, Jack Ferris, Anthony Giardullo, Sachin Kulkarni, Harry C Li, et al. TAO: Facebook's Distributed Data Store for the Social Graph. In USENIX Annual Technical Conference, pages 49-60, 2013.

[61] Austin Haugen. The open graph protocol design decisions. In International Semantic Web Conference, pages 338-338. Springer, 2010.

[62] The Open Graph protocol. http://ogp.me/.

[63] Sören Auer, Christian Bizer, Georgi Kobilarov, Jens Lehmann, Richard Cyganiak, and Zachary Ives. Dbpedia: A nucleus for a web of open data. The semantic web, pages 722-735, 2007.

[64] Virtuoso SPARQL Query Editor. http://dbpedia.org/sparql.

[65] Dario Bonino and Fulvio Corno. DogOnt-ontology modeling for intelligent domotic environments. The Semantic Web-ISWC 2008, pages 790-803, 2008.

[66] Dario Bonino, Emiliano Castellina, and Fulvio Corno. The DOG gateway: enabling ontology-based intelligent domotic environments. IEEE transactions on consumer electronics, 54(4), 2008.

[67] D. Bonino, E. Castellina, and F. Corno. Automatic domotic device interoperation. IEEE Transactions on Consumer Electronics, 55(2):499-506, May 2009.

[68] Dario Bonino and Giuseppe Procaccianti. Exploiting semantic technologies in smart environments and grids: Emerging roles and case studies. Science of Computer Programming, 95:112-134, 2014.

[69] Dario Bonino, Fulvio Corno, and Luigi De Russis. A semantics-rich information technology architecture for smart buildings. Buildings, 4(4):880-910, 2014.

[70] Michael Compton, Payam Barnaghi, Luis Bermudez, RaúL GarcíA-Castro, Oscar Corcho, Simon Cox, John Graybeal, Manfred Hauswirth, Cory Henson, Arthur Herzog, et al. The SSN ontology of the W3C semantic sensor network incubator group. Web semantics: science, services and agents on the World Wide Web, 17:25-32, 2012. 
[71] Semantic Sensor Network Ontology. https://www.w3.org/TR/vocab-ssn/.

[72] Victor Charpenay, Sebastian Käbisch, and Harald Kosch. Introducing Thing Descriptions and Interactions: An Ontology for the Web of Things. In Proceedings of the 1st Workshop on SemanticWeb technologies for the Internet of Things (SWIT) at the 15th International Semantic Web Conference (ISWC), 2016.

[73] IoT-Lite Ontology. http://iot.ee.surrey.ac.uk/fiware/ontologies/iot-lite.

[74] WoT ontology. http://iot.linkeddata.es/def/wot/index-en.html.

[75] W3C Interest Group on the Web of Things. http://w3c.github.io/wot/.

[76] WoT Current Practices. http://w3c.github.io/wot/current-practices/wot-practices.html.

[77] Dan Brickley and Libby Miller. FOAF vocabulary specification 0.91. Technical report, Citeseer, 2007.

[78] FOAF Vocabulary Specification. http://xmlns.com/foaf/spec/.

[79] B. Leiba. OAuth Web Authorization Protocol. IEEE Internet Computing, 16(1):74-77, Jan 2012.

[80] RFC 5849-The OAuth 1.0 Protocol. https://tools.ietf.org/html/rfc5849.

[81] RFC 6749-The OAuth 2.0 Authorization Framework. https://tools.ietf.org/html/ rfc6749.

[82] OAuth 2.0-OAuth. https://oauth.net/2/.

[83] Vipul Goyal, Omkant Pandey, Amit Sahai, and Brent Waters. Attribute-based encryption for fine-grained access control of encrypted data. In Proceedings of the 13th ACM conference on Computer and communications security, pages 89-98. Acm, 2006.

[84] RFC 7519-JSON Web Token (JWT). https://tools.ietf.org/html/rfc7519.

[85] RFC 6750-The OAuth 2.0 Authorization Framework: Bearer Token Usage. https: //tools.ietf.org/html/rfc6750.

[86] Guillermo del Campo, Eduardo Montoya, Jorge Martín, Igor Gómez, and Asunción Santamaría. BatNet: a 6LoWPAN-Based sensors and actuators network. In Ubiquitous Computing and Ambient Intelligence, pages 58-65. Springer, 2012.

[87] Arduino-Home. https://www.arduino.cc/.

[88] Zigduino-Logos Electromechanical LLC. http://www.logos-electro.com/zigduino/.

[89] IEEE Standard for Low-Rate Wireless Networks. IEEE Std 802.15.4-2015 (Revision of IEEE Std 802.15.4-2011), pages 1-709, April 2016.

[90] Nandakishore Kushalnagar, Gabriel Montenegro, and Christian Schumacher. IPv6 over low-power wireless personal area networks (6LoWPANs): overview, assumptions, problem statement, and goals. Technical report, 2007. 
[91] RFC 2460-Internet Protocol, Version 6 (IPv6) Specification. https://tools.ietf.org/html/ rfc2460.

[92] RFC 6550-RPL: IPv6 Routing Protocol for Low-Power and Lossy Networks. https: //tools.ietf.org/html/rfc6550.

[93] RFC 768-User Datagram Protocol. https://tools.ietf.org/html/rfc768.

[94] Best Practices for HTTP-CoAP Mapping Implementation. https://tools.ietf.org/html/ draft-castellani-core-http-mapping-06.

[95] RFC 7252-The Constrained Application Protocol (CoAP). https://tools.ietf.org/html/ rfc7252.

[96] Bernard Horan. The Use of Capability Descriptions in a Wireless Transducer Network. Technical report, Mountain View, CA, USA, 2005.

[97] Jorge M Perandones, Guillermo del Campo Jiménez, Jaime Caffarel Rodríguez, Song Jie, Silvia Calatrava Sierra, Rocío Martínez García, and Asunción Santamaría. Energysaving smart street lighting system based on 6LoWPAN. In Proceedings of the First International Conference on IoT in Urban Space, pages 93-95. ICST (Institute for Computer Sciences, Social-Informatics and Telecommunications Engineering), 2014.

[98] Jaime Caffarel, Song Jie, Jorge Olloqui, and Rocío Martínez. Bat-MP: an ontologybased energy management platform. In Ubiquitous Computing and Ambient Intelligence, pages 9-16. Springer, 2012.

[99] Parameter I Definition of Parameter by Merriam-Webster. https://www. merriam-webster.com/dictionary/parameter.

[100] Christian Bizer and Andreas Schultz. The berlin sparql benchmark, 2009.

[101] Anuradha Gali, Cindy X Chen, Kajal T Claypool, and Rosario Uceda-Sosa. From ontology to relational databases. In International Conference on Conceptual Modeling, pages 278-289. Springer, 2004.

[102] Core J2EE Patterns-Data Access Object. http://www.oracle.com/technetwork/java/ dataaccessobject-138824.html.

[103] Generic Types (The Java ${ }^{\mathrm{TM}}$ Tutorials $>$ Learning the Java Language $>$ Generics (Updated)). http://docs.oracle.com/javase/tutorial/java/generics/types.html.

[104] Trail: The Reflection API (The Java ${ }^{\mathrm{TM}}$ Tutorials). http://docs.oracle.com/javase/ tutorial/reflect/.

[105] Lesson: Annotations (The Java ${ }^{\mathrm{TM}}$ Tutorials > Learning the Java Language). https: //docs.oracle.com/javase/tutorial/java/annotations/.

[106] Dare Obasanjo. A comparison of Microsoft's C\# programming language to Sun Microsystem's Java programming language, 2002. 
[107] Jie Song, S. Calatrava Sierra, J. Caffarel Rodriguez, J. Martin Perandones, G. del Campo Jimenez, J. Olloqui Bujan, R. Martinez Garcia, and A. Santamaria Galdon. Data consistency management in an open smart home management platform. In Modelling Symposium (EMS), 2014 European, pages 366-371, Oct 2014.

[108] Green Labs|CeDInt-UPM. http://www.cedint.upm.es/en/project/green-labs.

[109] Danh Le-Phuoc, Hoan Nguyen Mau Quoc, Quoc Hung Ngo, Tuan Tran Nhat, and Manfred Hauswirth. Enabling live exploration on the graph of things. Proceedings of the Semantic Web Challenge, 2014.

[110] Mordechai Haklay and Patrick Weber. Openstreetmap: User-generated street maps. IEEE Pervasive Computing, 7(4):12-18, 2008.

[111] Sören Auer, Jens Lehmann, and Sebastian Hellmann. Linkedgeodata: Adding a spatial dimension to the web of data. In International Semantic Web Conference, pages 731-746. Springer, 2009.

[112] Developers/Mapbox. https://www.mapbox.com/developers/. (Accessed on 02/17/2017).

[113] Google Maps APIs|Google Developers. https://developers.google.com/maps/. (Accessed on 02/22/2017).

[114] Home-schema.org. http://schema.org/.

[115] Apache Jena. Reasoners and rule engines: Jena inference support. The Apache Software Foundation, 2013.

[116] Carlo Wouters, Tharam S Dillon, Wenny Rahayu, Robert Meersman, and Elizabeth Chang. Extraction process specification for materialized ontology views. In Advances in Web Semantics I, pages 130-175. Springer, 2009.

[117] Raphael Volz, Steffen Staab, and Boris Motik. Incremental maintenance of materialized ontologies. In On The Move to Meaningful Internet Systems 2003: CoopIS, DOA, and $O D B A S E$, pages 707-724. Springer, 2003.

[118] Ninghui Li. Discretionary access control. In Encyclopedia of Cryptography and Security, pages 353-356. Springer, 2011.

[119] Sylvia Osborn, Ravi Sandhu, and Qamar Munawer. Configuring role-based access control to enforce mandatory and discretionary access control policies. ACM Transactions on Information and System Security (TISSEC), 3(2):85-106, 2000.

[120] Gail-Joon Ahn. Discretionary Access Control. In Encyclopedia of Database Systems, pages 864-866. Springer, 2009.

[121] Ravi S Sandhu. Role-based access control. Advances in computers, 46:237-286, 1998.

[122] David Ferraiolo, D Richard Kuhn, and Ramaswamy Chandramouli. Role-based access control. Artech House, 2003. 
[123] Vincent C Hu, D Richard Kuhn, and David F Ferraiolo. Attribute-based access control. Computer, 48(2):85-88, 2015.

[124] Eric Yuan and Jin Tong. Attributed based access control (ABAC) for web services. In Web Services, 2005. ICWS 2005. Proceedings. 2005 IEEE International Conference on. IEEE, 2005.

[125] Tom Berners-Lee. Cool uris don't change. w3c, 1998.

[126] 29 Building RESTful Web Services with JAX-RS (Release 7). https://docs.oracle. $\mathrm{com} / \mathrm{javaee} / 7 /$ tutorial/jaxrs.htm.

[127] Air conditioning and refrigeration for residential, commercial and industrial applicationsl daikin global. http://www.daikin.com/products/ac/.

[128] WeChat Common Message. http://admin.wechat.com/wiki/index.php?title=Common_ Messages.

[129] Carriots-internet of things platform I home. https://www.carriots.com/.

[130] Sofia2 I home. http://sofia2.com/.

[131] Fiware. https://www.fiware.org/. (Accessed on 09/05/2017).

[132] Ibm watson-build your cognitive business with ibm. https://www.ibm.com/watson/. (Accessed on 09/05/2017). 


\section{Appendix A}

\section{Enumerations in BATOnt}

In this appendix, the predefined types for bat:Category, bat:ParameterType and bat:SpaceType are listed. Listing. A.1 presents the namespaces used in the following documents.

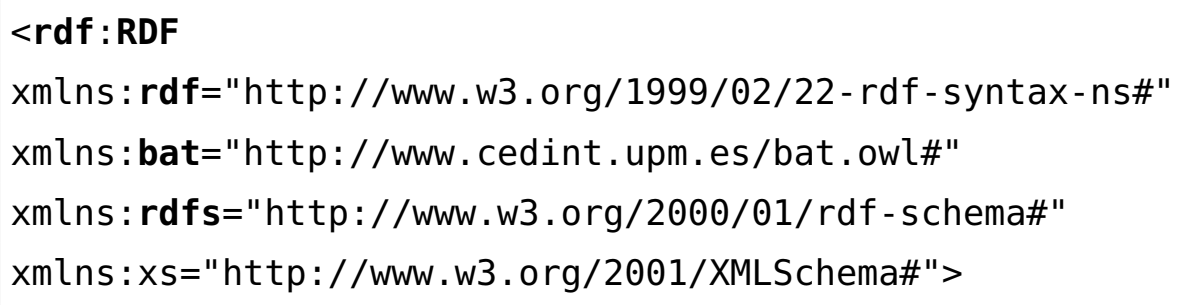

Listing A.1 Definition of namespaces

\section{Enumeration for Category}

Listing. A.2 presents the elements of the bat:Category enumeration that has been defined by BATOnt. 


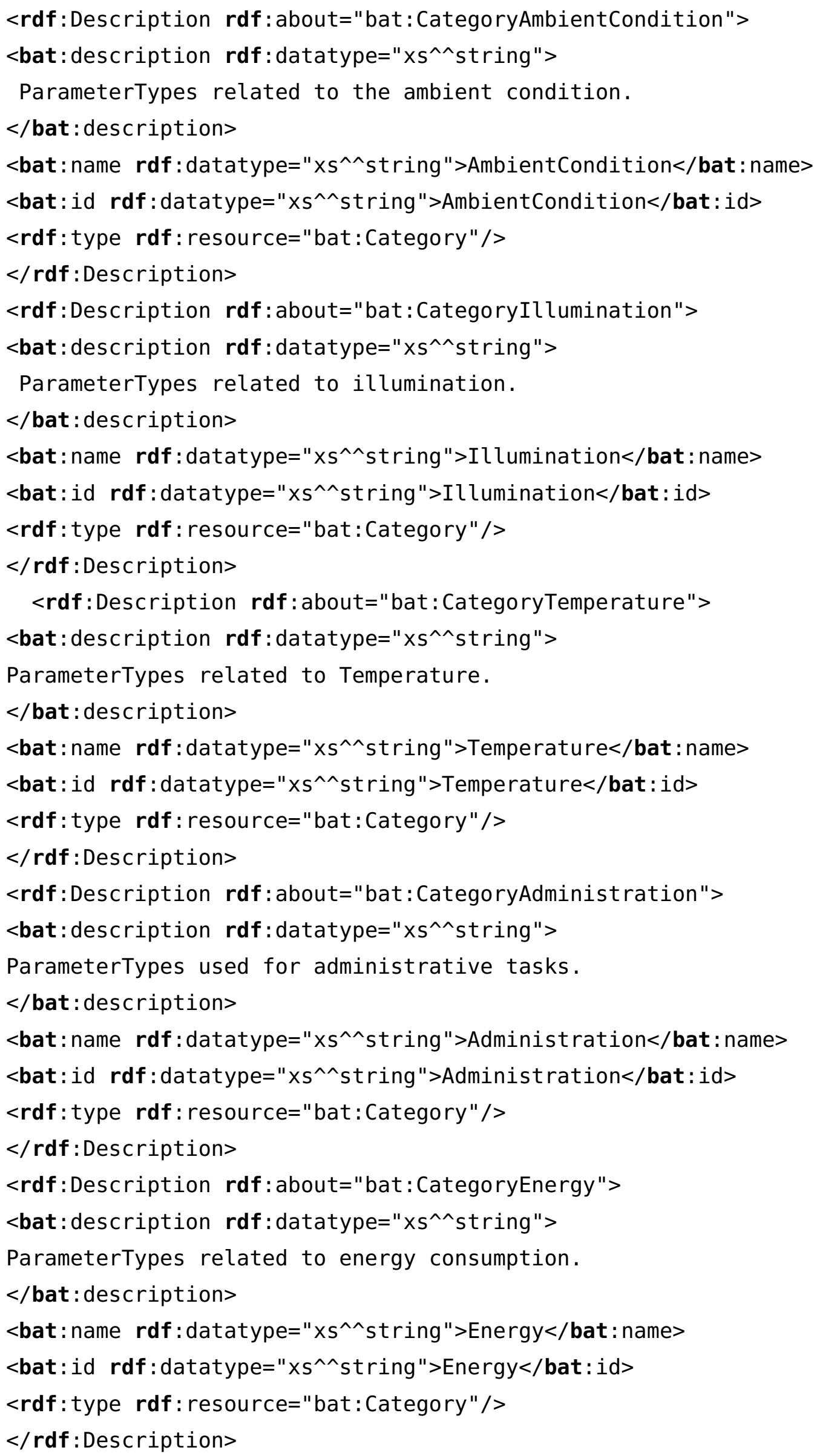




\section{Enumeration for ParameterType}

The elements of the ParameterType enumeration that has been defined by BATOnt are presented in Listing. A.3, Listing. A.4, Listing. A.5,Listing. A.6, Listing. A.7.

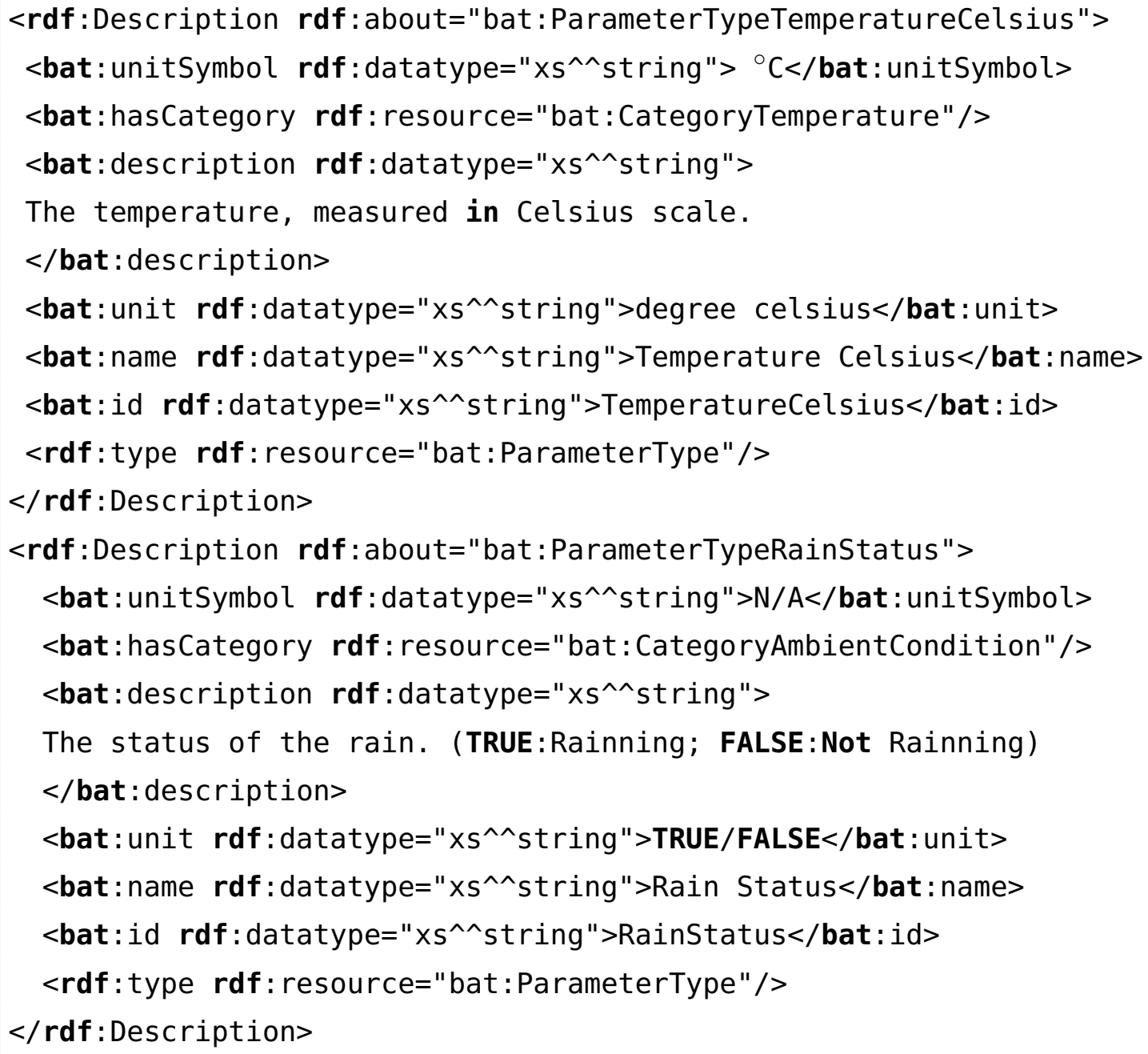

Listing A.3 Elements of the bat:ParameterType enumeration 


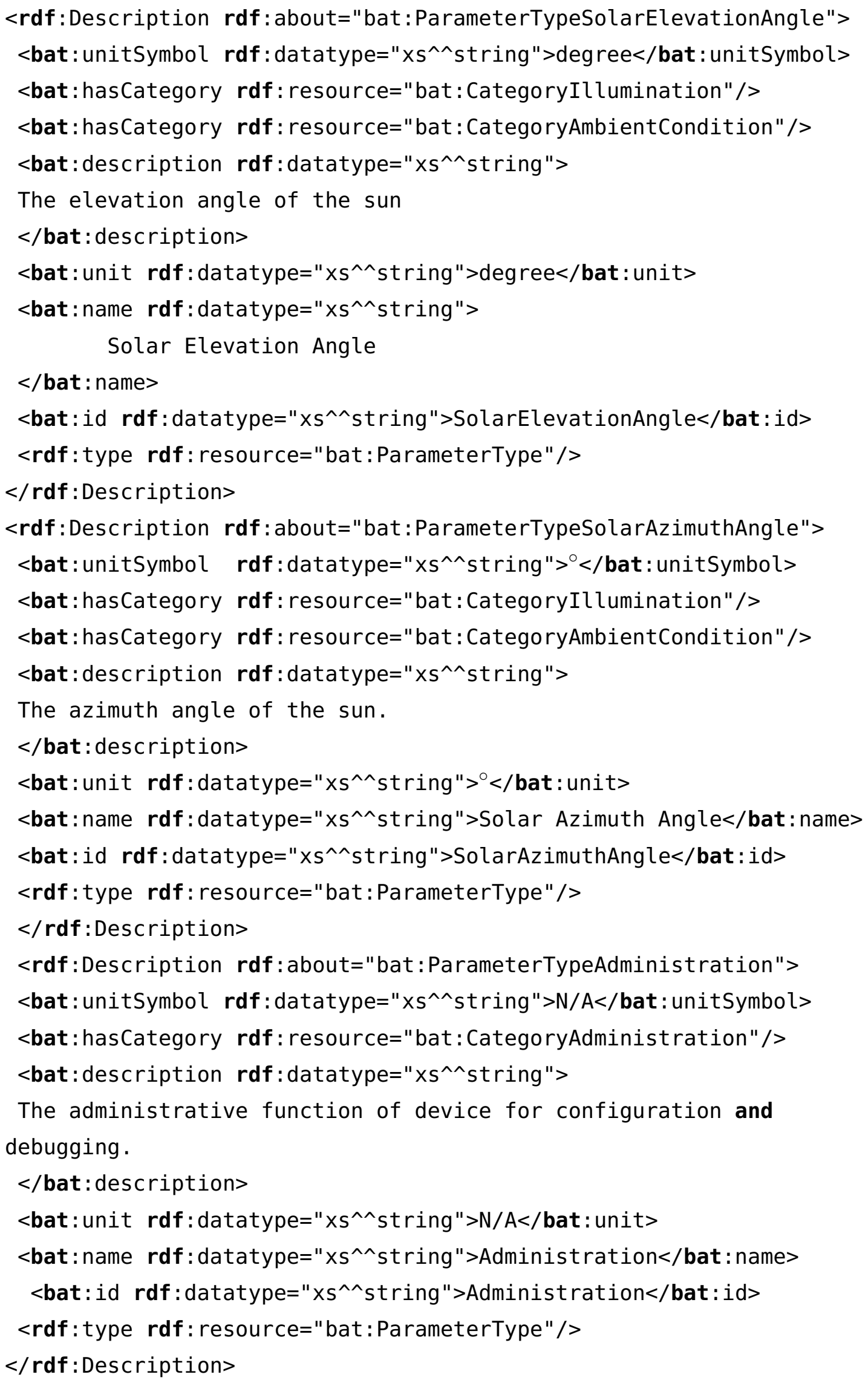




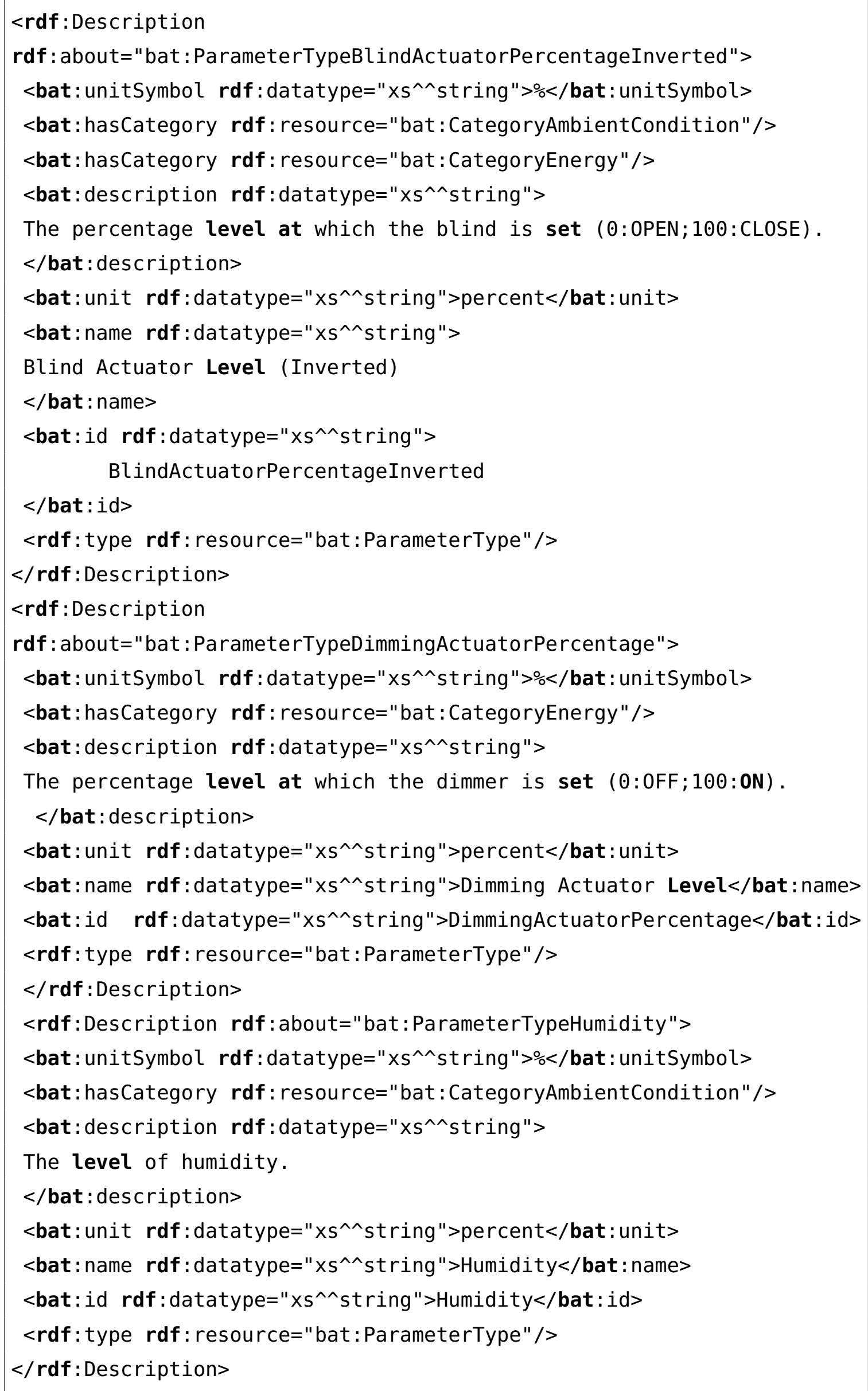

Listing A.5 Elements of the bat:ParameterType enumeration 


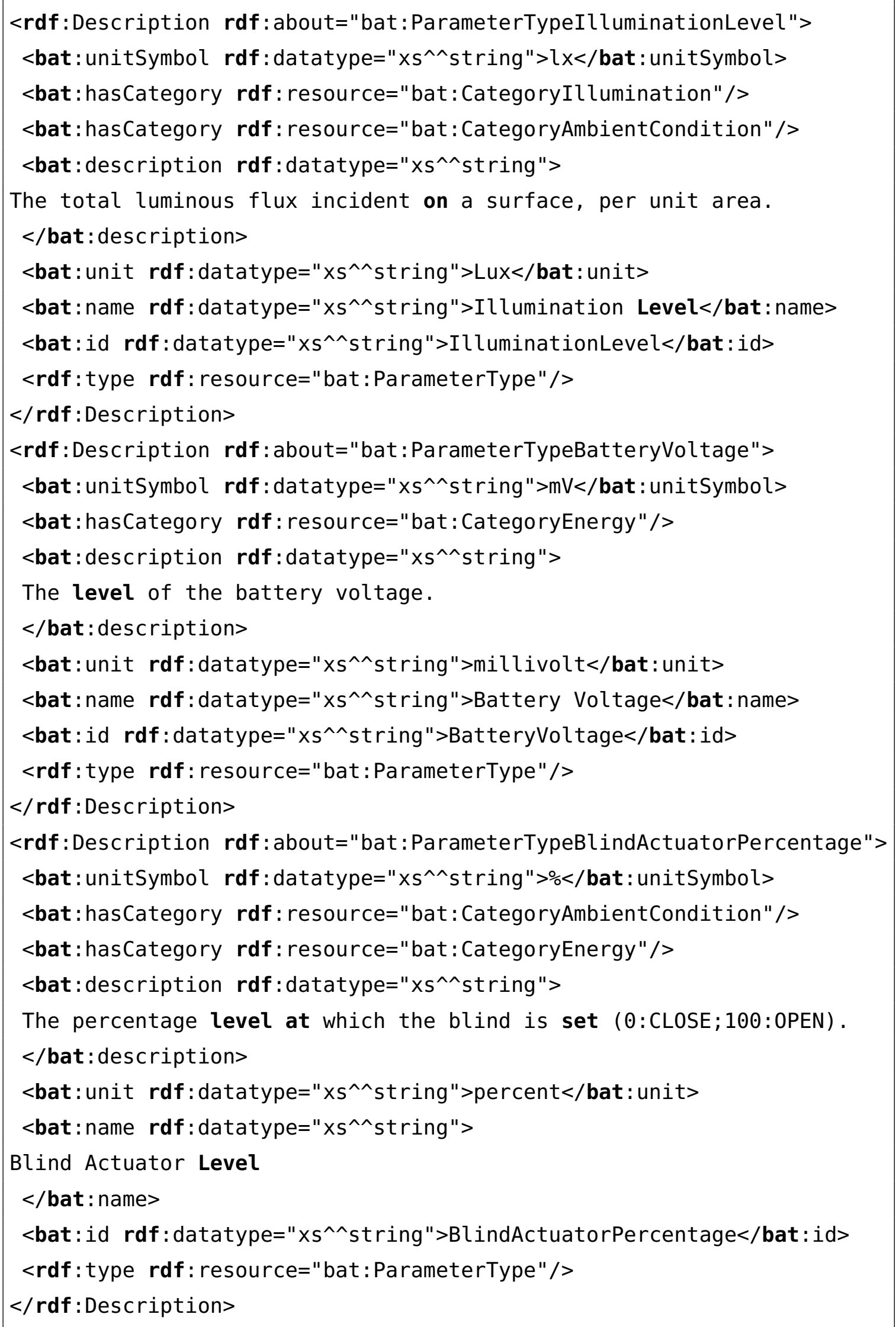

Listing A.6 Elements of the bat:ParameterType enumeration 


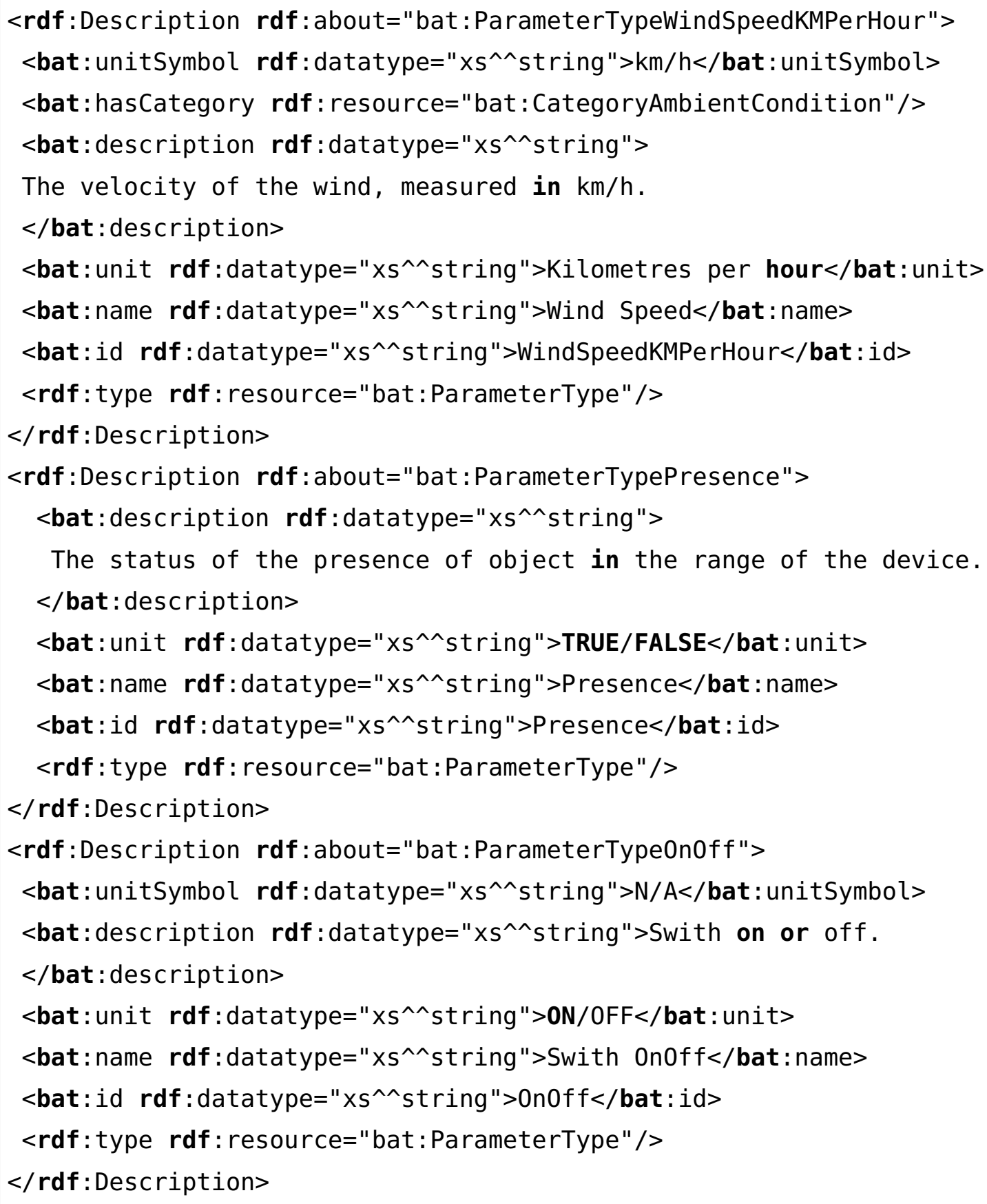

Listing A.7 Elements of the bat:ParameterType enumeration

\section{Enumeration for SpaceType}

The elements of the SpaceType enumeration that has been defined by BATOnt are presented in Listing. A.8, Listing. A.9, Listing. A.10,Listing. A.11, Listing. A.12, Listing. A.13, 
Listing. A.14, Listing. A.15, Listing. A.16, Listing. A.17, Listing. A.18.

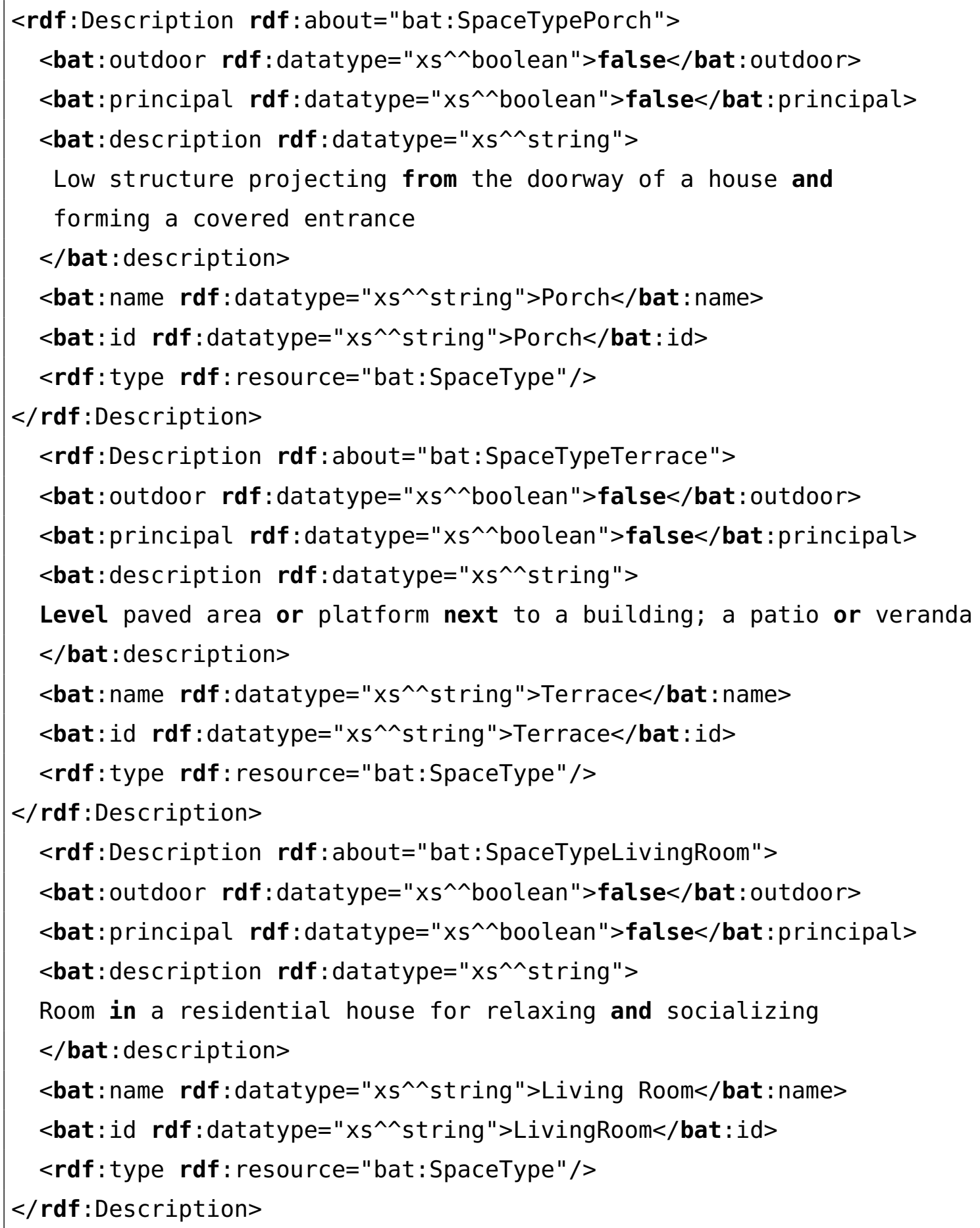

Listing A.8 Elements of the bat:SpaceType enumeration 


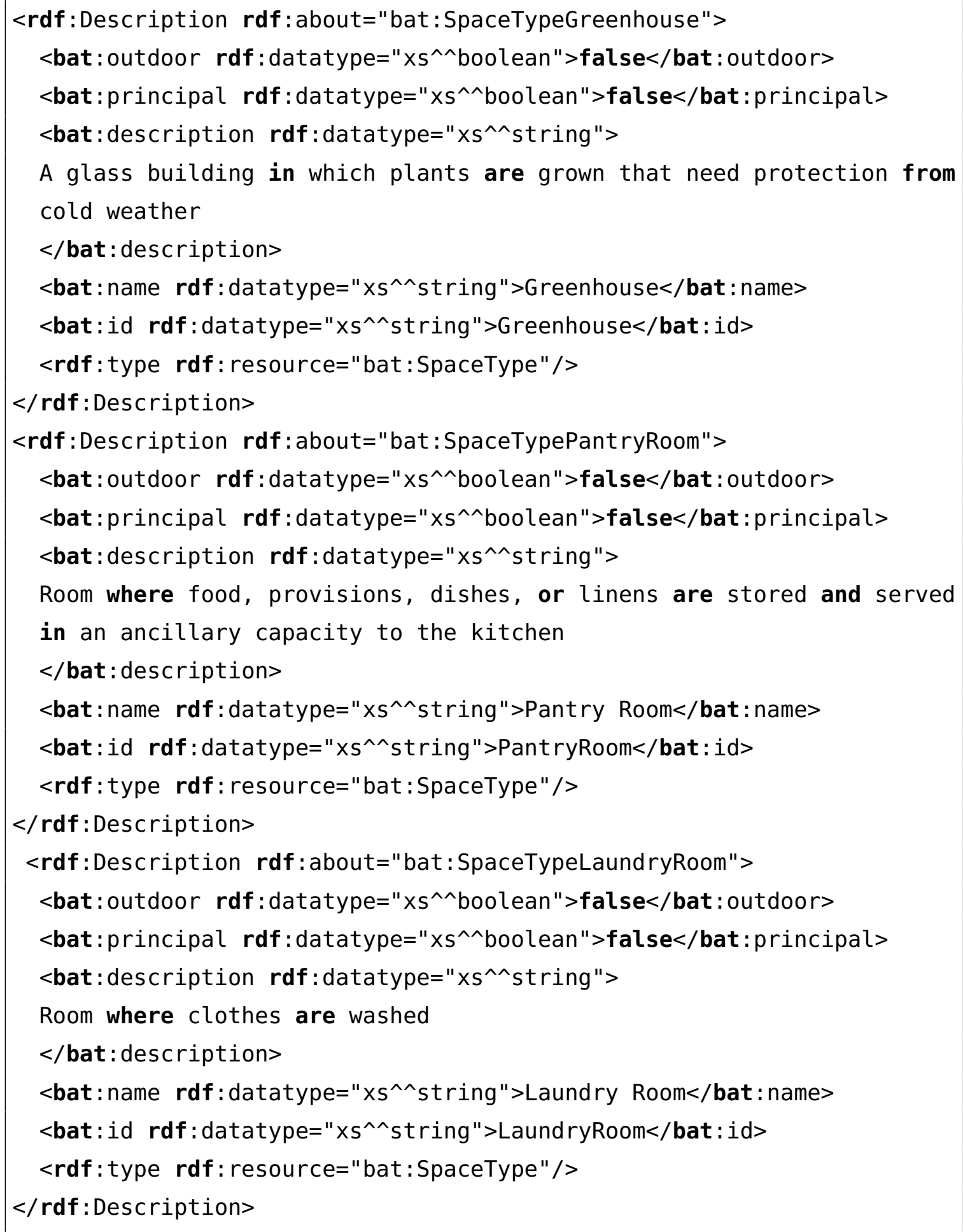

Listing A.9 Elements of the bat:SpaceType enumeration 


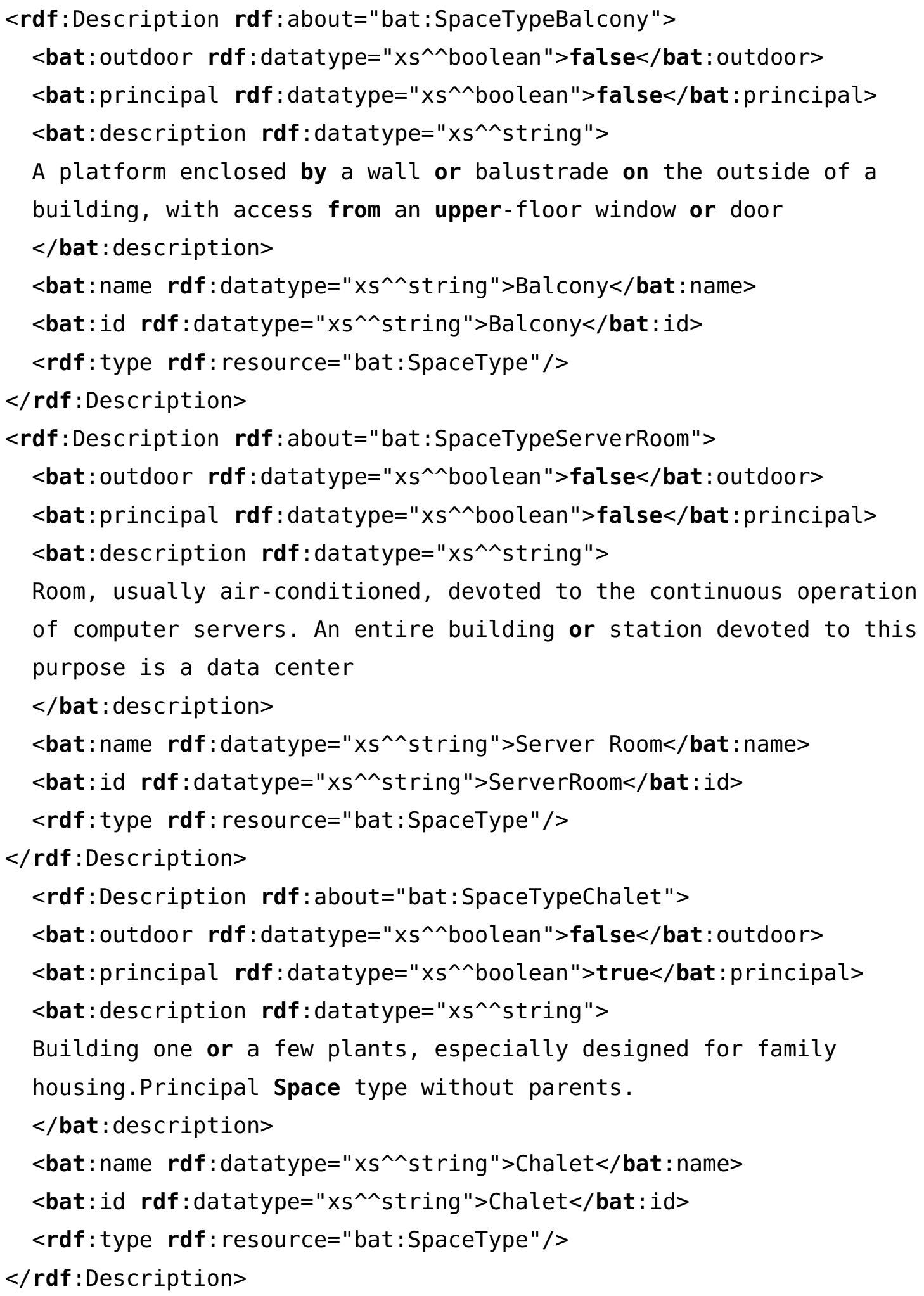

Listing A.10 Elements of the bat:SpaceType enumeration 
$<$ rdf:Description rdf:about="bat:SpaceTypeEntryway">

<bat: outdoor rdf: datatype="xs^^boolean">false</bat: outdoor $>$

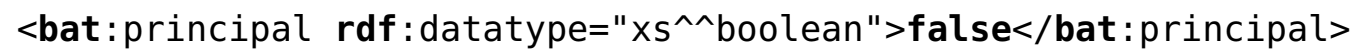
<bat: description rdf: datatype="xs^^string"> Hall that is generally located at the front entrance of a house $<$ bat: description $>$ $<$ bat: name rdf: datatype="xs^^string" $>$ Entryway</bat: name> $<$ bat: id rdf: datatype="xs^^string" $>$ Entryway $</$ bat $:$ id $>$ $<$ rdf: type rdf: resource="bat:SpaceType"/> $</$ rdf : Description $>$

$<$ rdf:Description rdf:about="bat:SpaceTypeControlRoom">

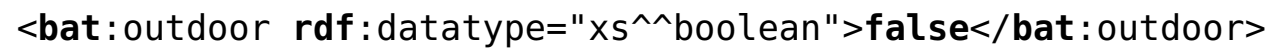
$<$ bat: principal rdf: datatype=" $x \mathrm{~s}^{\wedge \wedge}$ boolean" $>$ false $</$ bat: principal $>$ <bat: description rdf: datatype="xs^^string"> Room serving as a central space where a large physical facility or physically dispersed service can be monitored and controlled $</$ bat: description $>$ <bat: name rdf: datatype="xs^^string">Control Room</bat: name $>$

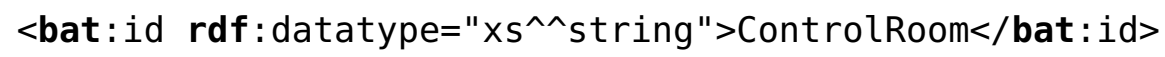
$<$ rdf: type rdf: resource="bat:SpaceType" /> $</$ rdf : Description $>$ $<$ rdf:Description rdf:about="bat:SpaceTypeLaboratory"> <bat: outdoor rdf: datatype="xs^^boolean">false</bat: outdoor $>$ $<$ bat: principal rdf: datatype=" $x \mathrm{~s}^{\wedge}$ boolean" $>$ false $</$ bat: principal $>$ <bat: description rdf: datatype=" $x \mathrm{~s}^{\wedge \wedge}$ string"> A room or building equipped for scientific experiments, research, or teaching, or for the manufacture of drugs or chemicals $<$ bat: description> $<$ bat: name rdf: datatype="xs^^string" $>$ Laboratory</bat: name $>$ $<$ bat: id rdf: datatype="xs^^string" $>$ Laboratory</bat: id $>$ <rdf: type rdf: resource="bat:SpaceType" /> $</$ rdf : Description $>$

Listing A.11 Elements of the bat:SpaceType enumeration 
<rdf:Description rdf:about="bat:SpaceTypeMeetingRoom"> <bat: outdoor rdf: datatype="xs^^boolean">false</bat: outdoor $>$ $<$ bat:principal rdf: datatype=" $x \mathrm{~s}^{\wedge}$ boolean" $>$ false $</$ bat: principal $>$ <bat: description rdf: datatype="xs^^string"> Room provided for meeting

$<$ /bat: description>

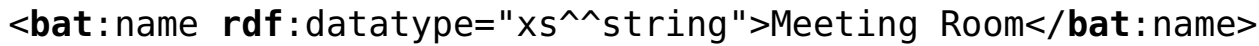
$<$ bat: id rdf: datatype="xs^^string" $>$ MeetingRoom</bat: id $>$ $<$ rdf: type rdf: resource="bat:SpaceType"/> $</$ rdf : Description $>$ $<$ rdf:Description rdf:about="bat:SpaceTypePatio">

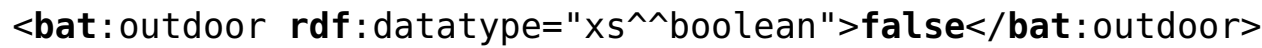
$<$ bat:principal rdf:datatype=" $x \mathrm{~s}^{\wedge}$ boolean" $>$ false $</$ bat: principal $>$ <bat: description rdf: datatype="xs^^string"> A paved outdoor area adjoining a house $</$ bat: description $>$ $<$ bat: name rdf:datatype=" $\mathrm{s}^{\wedge \wedge}$ string">Patio</bat: name> $<$ bat:id rdf: datatype="xs^^string">Patio</bat:id> $<$ rdf: type rdf: resource="bat:SpaceType" /> $</$ rdf : Description $>$ $<$ rdf:Description rdf:about="bat:SpaceTypeConferenceHall "> <bat: outdoor rdf: datatype=" $x s^{\wedge}$ boolean" $>$ false $</$ bat : outdoor $>$ $<$ bat:principal rdf:datatype=" $\mathrm{s}^{\wedge \wedge}$ boolean" $>$ false $</$ bat: principal $>$ <bat: description rdf: datatype="xs^^string"> Room provided for singular events such as business conferences $</$ bat: description $>$ $<$ bat: name rdf:datatype="xs^^string">Conference Hall</bat: name>

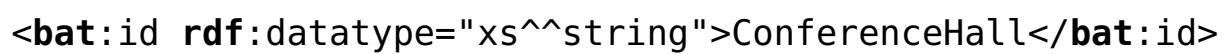
$<$ rdf: type rdf: resource="bat:SpaceType"/>

$</$ rdf : Description $>$

<rdf:Description rdf:about="bat:SpaceTypeStreet">

$<$ bat: outdoor rdf: datatype="xs^^boolean">true</bat: outdoor $>$ $<$ bat:principal rdf:datatype="xs^^boolean">true</bat:principal> <bat: description rdf: datatype="xs^^string">

In a population, road between buildings or land.

Principal Space type without parents.

$<$ bat: description>

$<$ bat: name rdf: datatype=" $\mathrm{x}^{\wedge \wedge}$ string" $>$ Street $</$ bat : name $>$

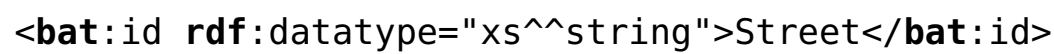

$<$ rdf: type rdf: resource="bat: SpaceType" />

$</$ rdf : Description $>$

Listing A.12 Elements of the bat:SpaceType enumeration 
<rdf:Description rdf:about="bat:SpaceTypeBathroom">

<bat: outdoor rdf: datatype="xs^^boolean">false</bat: outdoor $>$

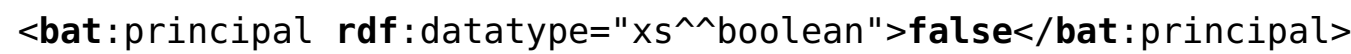
<bat: description rdf: datatype="xs^^string">

Room for personal hygiene

$<$ bat: description $>$

$<$ bat: name rdf: datatype="xs^^string" $>$ Bathroom</bat: name>

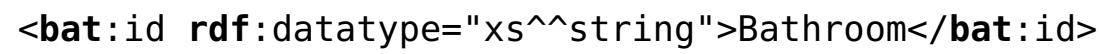

$<$ rdf: type rdf: resource="bat:SpaceType" />

$</$ rdf : Description $>$

<rdf:Description rdf:about="bat:SpaceTypeGarden">

<bat: outdoor rdf: datatype="xs^^boolean" $>$ false $</$ bat: outdoor $>$

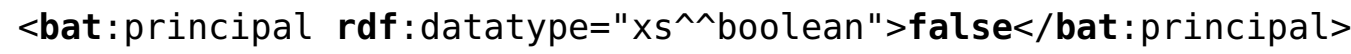

<bat: description rdf: datatype="xs^^string">

A piece of ground, often near a house, used for growing flowers, fruit, or vegetables

$<$ bat: description>

$<$ bat: name rdf: datatype="Xs^^string" $>$ Garden</bat: name>

$<$ bat: id rdf : datatype=" $x s^{\wedge \wedge}$ string" $>$ Garden $</$ bat $:$ id $>$

$<$ rdf: type rdf: resource="bat:SpaceType" $/>$

$</$ rdf : Description $>$

$<$ rdf:Description rdf:about="bat:SpaceTypeWarehouse">

<bat: outdoor rdf: datatype="xs^^boolean">false</bat: outdoor $>$

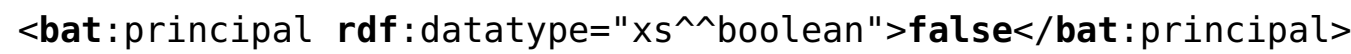

<bat: description rdf: datatype="xs^^string">

Place where goods are stored prior to their use, distribution, or sale

$<$ bat: description>

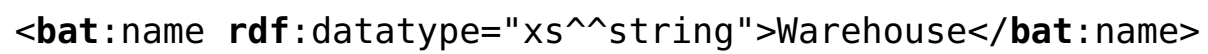

$<$ bat: id rdf: datatype="xs^^string" $>$ Warehouse $</$ bat $:$ id $>$

$<$ rdf: type rdf: resource="bat:SpaceType" />

$</$ rdf : Description $>$

$<$ rdf:Description rdf:about="bat:SpaceTypeInternalParking"> <bat: outdoor rdf: datatype="xs^^boolean">false</bat: outdoor $>$ $<$ bat: principal rdf: datatype $=" x s^{\wedge}$ boolean" $>$ false $</$ bat: principal $>$ $<$ bat: description rdf: datatype="xs^^string" $><$ bat: description> $<$ bat: name rdf:datatype="xs^^string" $>$ Internal Parking</bat: name> $<$ bat: id rdf: datatype="xs^^string">InternalParking</bat:id> $<$ rdf: type rdf: resource="bat:SpaceType"/>

$</$ rdf : Description $>$

Listing A.13 Elements of the bat:SpaceType enumeration 


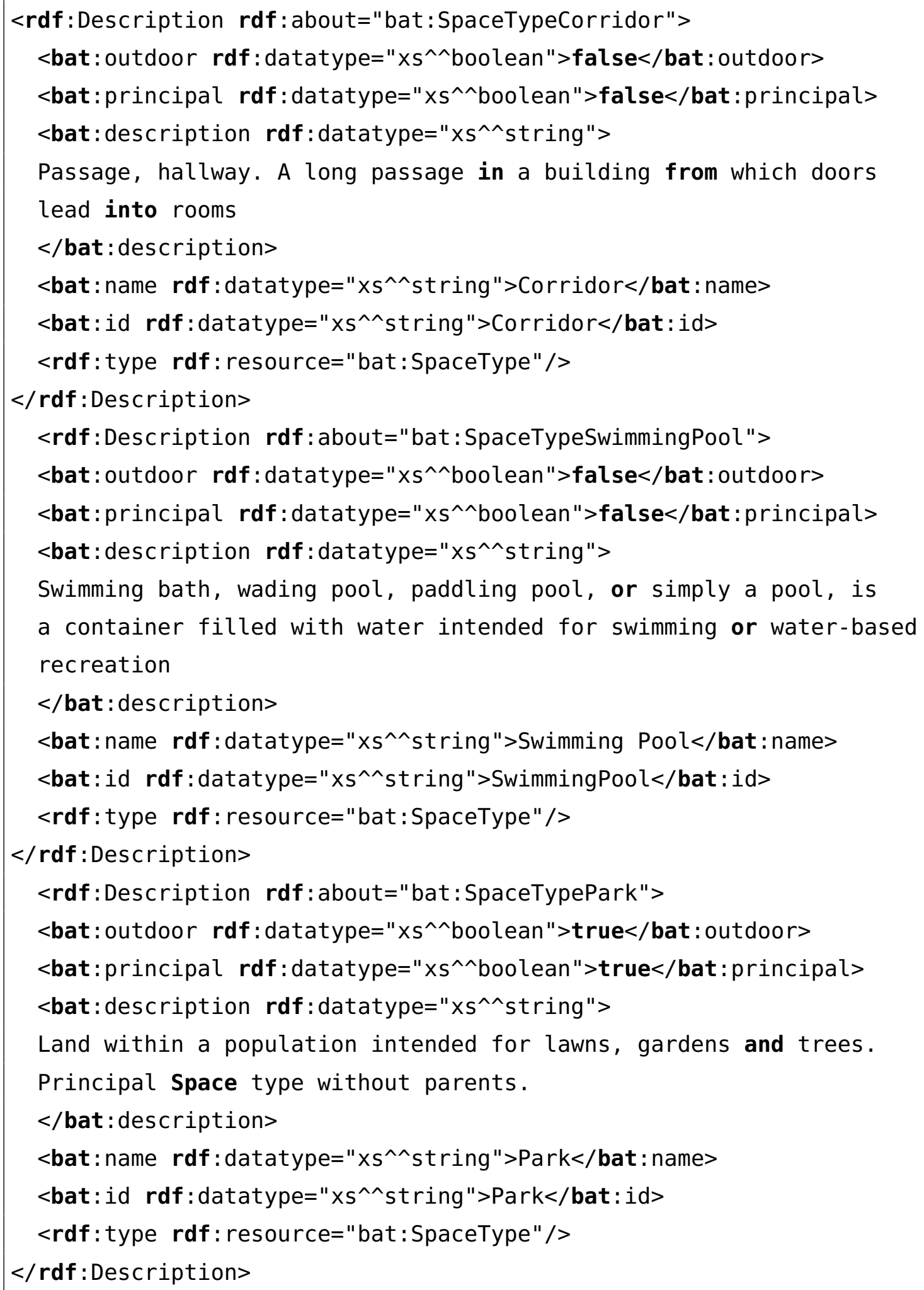

Listing A.14 Elements of the bat:SpaceType enumeration 
$<$ rdf:Description rdf:about="bat:SpaceTypeFlat">

<bat: outdoor rdf: datatype="xs^^boolean">false</bat: outdoor> $<$ bat: principal rdf: datatype="xs^^boolean">true</bat: principal $>$ <bat: description rdf: datatype="xs^^string">

Set up independent living rooms in a house of various heights. Principal Space type without parents.

$</$ bat: description $>$

$<$ bat: name $\mathbf{r d f}$ : datatype $=$ "Xs^^string" $>$ Flat $</$ bat: name $>$

$<$ bat: id rdf: datatype=" $x \mathrm{~s}^{\wedge \wedge}$ string" $>$ Flat $</$ bat : id $>$

<rdf: type rdf: resource="bat:SpaceType"/>

$</$ rdf : Description $>$

<rdf:Description rdf:about="bat:SpaceTypeShowroom">

<bat: outdoor rdf: datatype="xs^^boolean">false</bat: outdoor $>$

$<$ bat: principal rdf: datatype=" $x \mathrm{~s}^{\wedge}$ boolean" $>$ false $</$ bat: principal $>$ <bat: description rdf: datatype="xs^^string">

Space for demostrations and exhibitions.

$</$ bat: description>

$<$ bat: name rdf: datatype $=" x s^{\wedge \wedge}$ string" $>$ Showroom</bat: name $>$

$<$ bat: id rdf: datatype="xs^^string" $>$ Showroom</bat: id $>$

$<$ rdf: type rdf: resource="bat:SpaceType"/>

$</$ rdf : Description $>$

$<$ rdf:Description rdf:about="bat:SpaceTypeKitchen">

<bat: outdoor rdf: datatype="xs^^boolean">false</bat: outdoor $>$

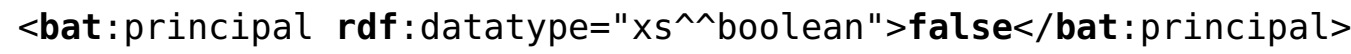

<bat: description rdf: datatype="xs^^string">

Room or part of a room used for cooking and food preparation

$</$ bat: description $>$

$<$ bat: name rdf:datatype="Xs^^string" $>$ Kitchen $</$ bat: name $>$

$<$ bat: id rdf: datatype="xs^^string">Kitchen</bat: id>

$<$ rdf:type rdf: resource="bat:SpaceType"/>

$</$ rdf : Description $>$

$<$ rdf:Description rdf:about="bat:SpaceTypePath">

<bat: outdoor rdf: datatype="xs^^boolean">false</bat: outdoor $>$

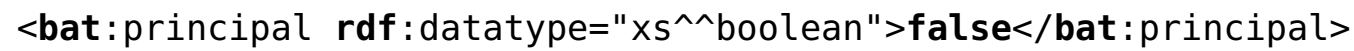

<bat: description rdf:datatype="xs^^string">

A way or track laid down for walking.

$</$ bat: description $>$

$<$ bat: name rdf: datatype="xs^^string" $>$ Path</bat: name>

$<$ bat: id rdf: datatype="xs^^string" $>$ Path</bat: id $>$

$<$ rdf: type rdf: resource="bat:SpaceType"/>

$</$ rdf : Description $>$

Listing A.15 Elements of the bat:SpaceType enumeration 


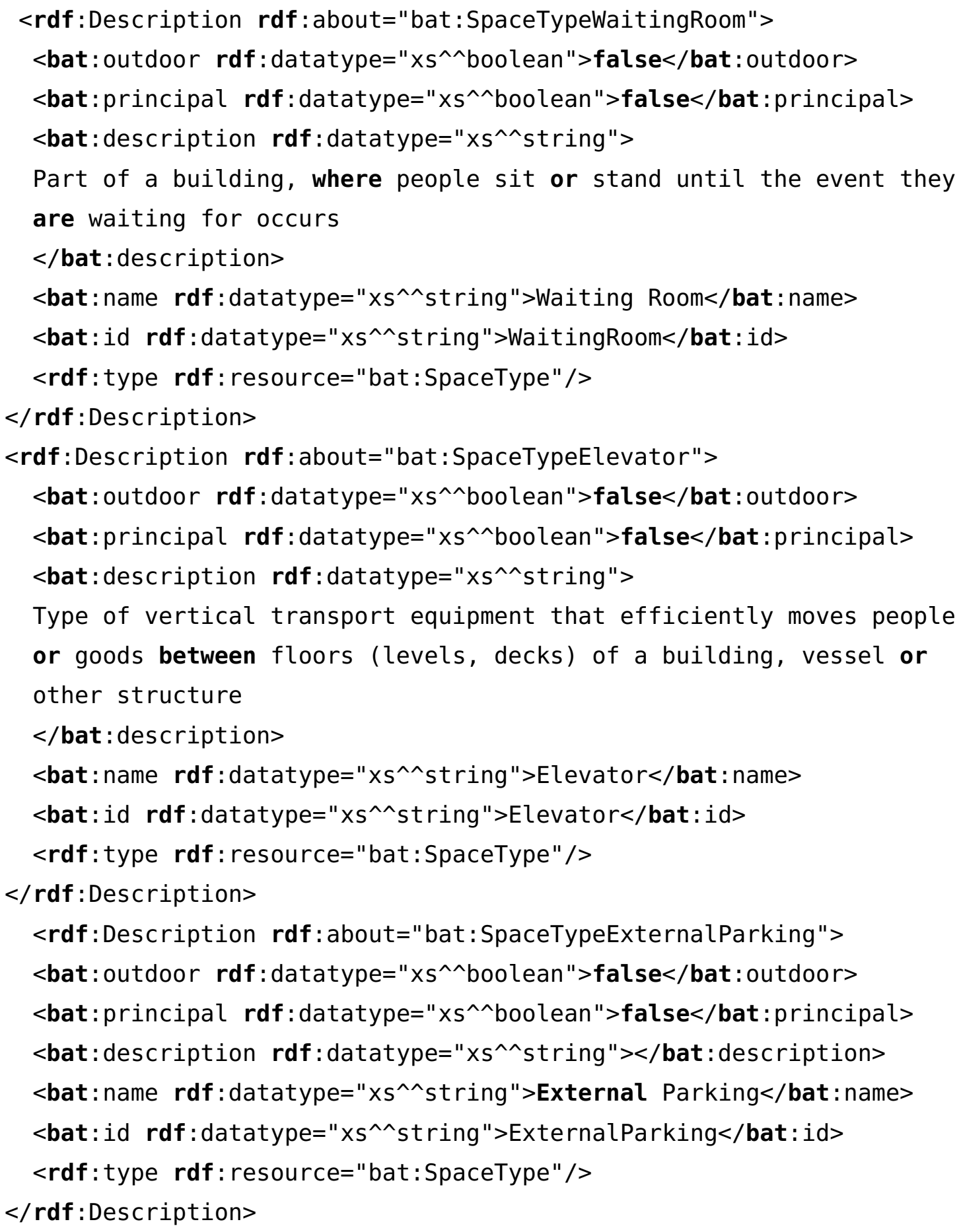

Listing A.16 Elements of the bat:SpaceType enumeration 


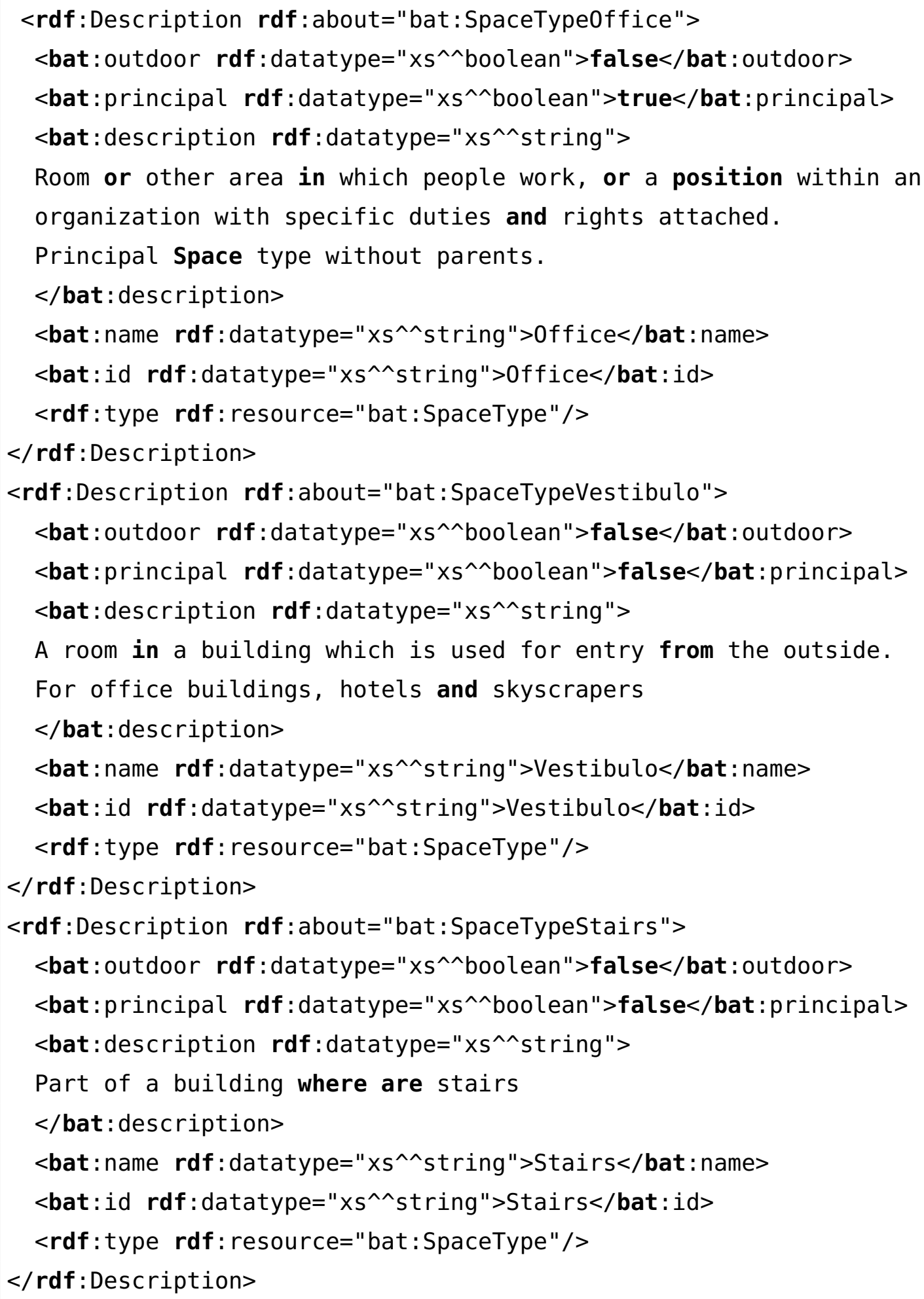

Listing A.17 Elements of the bat:SpaceType enumeration 


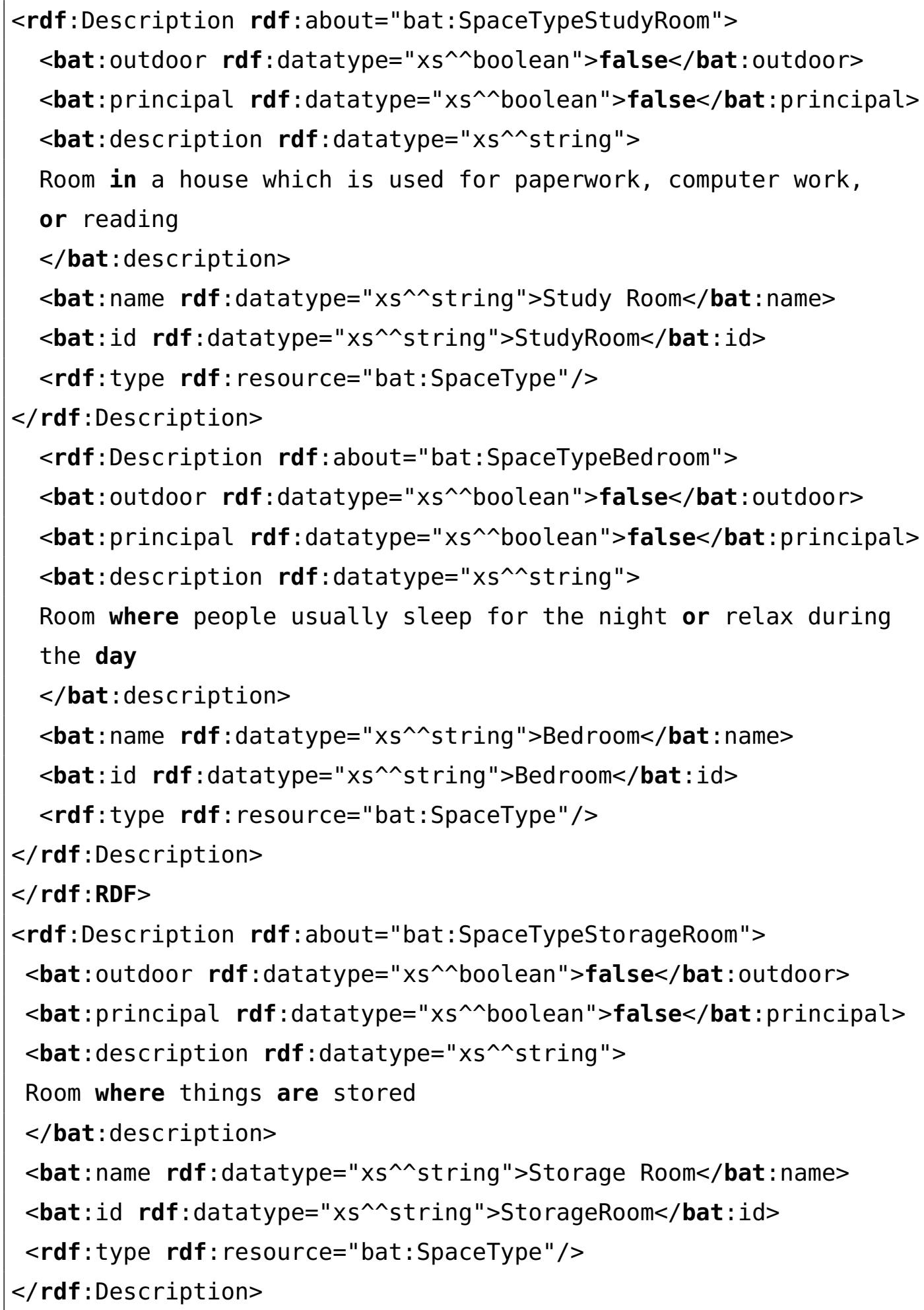

Listing A.18 Elements of the bat:SpaceType enumeration 